Skeletal muscle mass in head and neck cancer patients: radiological assessment and association with clinical outcome

\author{
Sandra Bril
}




\section{Skeletal muscle mass in head and neck cancer}

patients

Radiological assessment and association with clinical outcome

Skeletspiermassa bij hoofd-halskankerpatiënten

Radiologische beoordeling en relatie met klinische uitkomsten

(met een samenvatting in het Nederlands)

\section{Proefschrift}

\section{Colofon tekst}

\section{Author \\ Cover design by \\ Layout}

Printing

ISBN

The research as described was supported by the Michel Keijzer Fonds, a not for profit fund supporting research which aims to improve care for laryngectomees and other head and neck cancer patients.

All rights reserved. No part of this thesis may be reproduced, stored in a retrieval system of any nature, or transmitted in any form or by any means, without permission of the author or, when appropriate, of the publishers of the publications.

Copyright $\odot 2021$ Sandra Isabella Bril, Tilburg, The Netherlands
Sandra Isabella Bril Profschrift - AlO, wWw.proefschrift-aio.n proefschrift - AIO, www.proefschrift-aio.nl 978-94-93270-18-3 ter verkrijging van de graad van doctor aan de Universiteit Utrecht

op gezag van de rector magnificus, prof.dr. H.R.B.M. Kummeling, ingevolge het besluit van het college voor promoties

in het openbaar te verdedigen op

dinsdag 23 november 2021 des middags te 2.15 uur

doo

\section{Sandra Isabella Bri}

geboren op 19 oktober 1989 te Vries 


\section{Promotor:}

Prof. dr. R. de Bree

Copromotor:

Dr. L.A. Devriese

\section{Leden van de beoordelingscommissie:}

Prof. dr. P.O. Witteveen

Prof. dr. M.H. Emmelot-Vonk

Prof. dr. J. Hendrikse

Prof. dr. M.W.M. van den Brekel

Dr. R.J.H.M. Steenbakkers

Dit proefschrift werd mede mogelijk gemaakt met financiële steun vanuit het Michel Keijzer Fonds, een aan de patiëntenvereniging Hoofd-Hals gelieerd fonds dat projecten en onderzoeken ondersteunt die voor gelaryngectomeerden van belang kunnen zijn. 


\section{TABLE OF CONTENTS}

Part

Introduction

Chapter 1. General introduction and aims of the thesis

Chapter 10. A study protocol for an observational study investigating the importance of skeletal muscle mass for cisplatin pharmacokinetics in patients with head and neck cancer: the PLATISMA study

Part II Assessment of low skeletal muscle mass in head and neck cancer patients

muscle mass in head and neck cancer patients using

head and neck CT imaging

Chapter 3. Interobserver agreement of skeletal muscle mass

measurement on head and neck CT imaging at the level of the third cervical vertebra

Chapter 4. Skeletal muscle volume estimation from a single magnetic resonance imaging slice of the head and neck area in healthy adults

Part III Radiological assessment of body composition and clinical outcomes in head and neck cancer patients

Chapter 5. Pre-treatment low skeletal muscle mass is associated with chemotherapy dose-limiting toxicity in head and neck cancer patients undergoing primary chemoradiotherapy with cisplatin

Chapter 6. Pre-operative low skeletal muscle mass as a risk factor for pharyngocutaneous fistula and decreased overall survival in patients undergoing laryngectomy

Chapter 7. Sarcopenia is a prognostic factor for overall survival in elderly patients with head-and-neck cancer

Chapter 8. Skeletal muscle mass is an imaging biomarker for decreased survival in patients with oropharyngea squamous cell carcinoma

Chapter 9. Arterial calcification on preoperative $\mathrm{CT}$ imaging is a risk factor for pharyngocutaneous fistula formation after total laryngectomy
Appendices

Chapter 12. Dutch summary - Nederlandse samenvatting

Chapter 13. Curriculum vitae

Chapter 14. List of publications
Chapter $15 . \quad$ Dankwoord 
Part I

Introduction 
Chapter 1

\section{General introduction} and aims of the thesis

Adapted from:

Sandra I. Bril, Ajit J. Pothen, Remco de Bree. Sarcopenie bij patiënten met hoofdhalskanker. Nederlands Tijdschrift voor Oncologie. 2017; 14(2). 


\section{General introduction}

Head and neck cancers (HNCs) are among the most frequent tumors in the world with an estimated 835.000 new cases and 428.000 deaths in $2018 .^{2}$ There has been a significant increase in the global incidence of HNC over the past decade. In the Netherlands, about 3000 patients are diagnosed with HNC yearly, resulting in approximately 900 annual deaths. ${ }^{3}$ More than $90 \%$ of all HNCs are head and neck squamous cell carcinomas (HNSCC). Prolonged tobacco consumption and alcohol abuse are the main risk factors associated with HNSCC, and the combined consumption of alcohol and tobacco appears to have a synergistic effect. ${ }^{4}$ Besides these traditional risk factors, a specific subset of HNSCC predominantly located in the oropharynx is caused by high risk human papillomaviruses (HPV); commonly high-risk HPV types 16 and $18 .{ }^{5}$ The incidence of HPV-positive oropharyngeal cancer is fast growing, particularly in developed countries. Patients with HPV positive oropharyngeal cancer are typically younger, have less comorbidities and lack the traditional risk factors for head and neck cancer such as prolonged tobacco consumption or alcohol abuse.

\section{Curative treatment options for head and neck squamous cell carcinoma}

Early stage HNSCC can be treated curatively with primary radiotherapy or local surgical excision. The majority of HNSCC patients presents with advanced disease at diagnosis (large tumor and/or regional lymphatic metastasis). Distant metastasis at diagnosis is uncommon at approximately $10 \%$ of patients, so the treatment of advanced stage HNSCC is most often with curative intent. ${ }^{4}$ The treatment of advanced stage HNSCC is commonly multimodal, and generally includes primary surgery and adjuvant radiotherapy with or without chemotherapy; or primary radiotherapy with chemotherapy with salvage surgery in reserve. ${ }^{7}$ During the last two decades, the preference of first choice treatment of advanced stage HNSCC has generally shifted from primary surgery to primary radiotherapy combined with chemotherapy (chemoradiotherapy; CRT) with high dose cisplatin as the chemotherapeutical agent. ${ }^{8}$ The main benefit of primary CRT as opposed to primary surgery is that it offers the opportunity for organ preservation (e.g. larynx) and to generally avoid major and complex surgery. ${ }^{9}$ However, organ preservation is not similar to preservation of function, which must be the goa of organ preservation treatments. Because of better oncological outcome resectable T4 are generally treated by surgery. Irresectable HNSCC is of course treated by radiotherapy and/or chemotherapy. Other treatment options include targeted therapy, e.g. cetuximab, a monoclonal antibody targeting the epithelial growth factor receptor (EGFR), combined with radiotherapy. The combination of cetuximab and radiotherapy is less effective than platinum-based CRT, but is an option in platinum unfit patients ${ }^{10,11}$ For treatment decision making every HNC patient is discussed in a HNC multidisciplinary team (MDT).

\section{Chemoradiotherapy in head and neck squamous cell carcinoma}

Commonly, primary CRT with curative intent consists of radiotherapy with 70 Gray total radiation dose in 35 fractions, generally combined with 3 three-weekly cycles high dose cisplatin at $100 \mathrm{mg} / \mathrm{m}^{2}$ body surface area (BSA) per cycle, at a cumulative dose of $300 \mathrm{mg} / \mathrm{m} 2$ BSA of cisplatin. ${ }^{8}$ Cisplatin acts as a radiosensitizer in HNSCC, and the addition of high dose cisplatin chemotherapy to radiotherapy monotreatment improves locoregional disease control and results in a 6.5\% increase in 5-year overall survival. ${ }^{8}$ However, the addition of cisplatin to radiation treatment can cause severe side effects, such as nausea, nephrotoxicity, bone marrow depression, neurotoxicity and ototoxicity. Acute toxicity is a common and serious problem in HNSCC patients, and results in dose-reductions, treatment delay or treatment termination (chemotherapy dose limiting toxicity, CDLT) in approximately $30 \%$ of the patients. ${ }^{12}$ An alternative to the high dose, three-weekly cisplatin chemotherapeutical regimen is weekly cisplatin at a dose level of 30$40 \mathrm{mg} / \mathrm{m}^{2}$ which is generally associated with less acute toxicity and still results in a cumulative cisplatin dose of 210 to $280 \mathrm{mg} / \mathrm{m}^{2}$ BSA. Currently, randomized controlled trials have provided level 1 evidence for the improvement of locoregional control and overall survival in locally advanced HNSCC with primary CRT with 3 three-weekly cycles of high dose cisplatin at a dose level of 100mg/ m2 BSA. ${ }^{12}$ Prospective randomized controlled trials directly comparing high dose cisplatin with low dose cisplatin are however scarce. A systematic review of 52 prospective trials in head and neck cancer patients concluded that there is insufficient evidence available to meaningfully compare high dose cisplatin to low dose cisplatin. ${ }^{13}$ In recent years, several large clinical trials have investigated de-escalation strategies with cetuximab as radiosensitizer in HNSCC, particularly in HPV-associated HNSCC. ${ }^{14,15}$ These studies concluded that CRT with high-dose three-weekly cisplatin remains the preferred treatment option with the highest survival benefit compared to cetuximab.

It can be anticipated that CDLT has a negative effect on the survival of HNSCC patients because patients receive a suboptimal treatment with toxic side effects. Large prospective trials and retrospective studies show that a higher cumulative dose is associated with better survival rates, and particularly that a cumulative cisplatin dose of less than $200 \mathrm{mg} / \mathrm{m}^{2}$ is associated with a significantly decreased 
survival in HNSCC patients treated with CRT. ${ }^{16-19}$ Treatment-related toxicity itself can also have a negative effect on short-term and long-term survival. ${ }^{17}$ There are several contraindications for the use of high dose cisplatin, such as decreased renal function, severe hearing loss, poor WHO functional status, but these contraindications do not identify the $30 \%$ of patients that experience CDLT in daily clinically practice. There is a clinical need for new accurate predictive characteristics or biomarkers to identify patients at high risk of cisplatin doselimiting toxicity.

\section{Treatment of laryngeal cancer}

Specific for patients with advanced stage laryngeal or hypopharyngeal cancer treatment options include primary total laryngectomy, with or without (partial) pharyngectomy (pharyngo-laryngectomy), primary radiotherapy, and CRT. The latter two treatment options have the intent of organ preservation of the larynx. ${ }^{9}$ Treatment choice for either options may be guided by bulky tumor size or cartilage involvement (favoring primary laryngectomy), functional status and patient preferences. ${ }^{20,21}$ Despite initial organ preservation strategies, laryngectomy still needs to be performed in roughly a third of advanced stage laryngeal and pharyngeal cancer patients, either as salvage procedure for recurrent disease, as a treatment of a second primary malignancy or for functional reasons. ${ }^{22}$

Total laryngectomy is a major surgical procedure and is associated with significant morbidity and mortality, as well as a reduced quality of life after surgery. Postoperative complications, particularly the occurrence of a pharyngocutaneous fistula (PCF) are common and difficult to treat. Up to thirty percent of patients develop PCF, which may require additional reconstructive surgery, prolong feeding tube dependency and increase hospital stay. ${ }^{23-25}$ The occurrence of a PCF may also cause delay of postoperative (chemo)radiotherapy, thus jeopardizing optima oncological treatment. Previously described risk factors for the occurrence of a PCF include prior CRT with platinum-based chemotherapy, hypopharyngeal cancer, extensive pharyngeal resection and reconstruction, additional neck dissection and low body mass index. ${ }^{26}$ Current research aims to find additional risk factors for the occurrence of a PCF, to provide the clinician with accurate tools to identify those patients at highest risk of PCF. Possibly, preemptive treatment strategies or different surgical techniques can be used in those patients to decrease the risk of PCF. ${ }^{27}$

One risk factor proposed in recent oncological research for both the occurrence of postoperative complications as well as CDLT is an abnormal body composition and specifically low skeletal muscle mass, sometimes termed sarcopenia.

\section{Low skeletal muscle mass}

Over the last decade, the specific body composition of a patient has become subject of interest in oncological and surgical research. A person's body composition traditionally describes the amount of lean tissue mass and fat tissue mass in the body, and its proportionality to one other. The skeletal muscle mass is the largest contributor to the lean tissue mass. ${ }^{28} \mathrm{~A}$ deficit of skeletal muscle mass, sometimes termed sarcopenia, and particularly when combined a state of disproportionality surplus of fat mass (sarcopenic obesity), is associated with adverse outcomes in surgical and oncological literature. ${ }^{29-31}$

Sarcopenia lends its name from the Greek words "sarx" meaning flesh and "penia" meaning lack of. Sarcopenia was first described as a geriatric syndrome, describing the age-related loss of skeletal muscle mass while fat mass remains equal or increases, combined with a loss of muscle function. The official definition of sarcopenia of the European Working Group on Sarcopenia in Older People (EWGSOP) requires a decrease in skeletal muscle mass and a decrease in muscle function. ${ }^{32}$ In the elderly, sarcopenia is a risk factor for various adverse outcomes including physical disability, decreased quality of life, and ultimately early death It is estimated that the prevalence of sarcopenia in the general population is $5-13 \%$ for people aged $60-70$ years, and up to $50 \%$ for those aged 80 years or above. ${ }^{33}$ Independent of age, sarcopenia can exist secondary to chronic systemic inflammation, malnutrition and immobilization..$^{32}$ In cancer patients, a risk factor for sarcopenia inherently present is the malignant tumor itself, which may trigge a chronic systemic inflammatory process in the body as a reaction to the tumor. ${ }^{32}$

\section{Investigating body composition}

There are several methods to investigate the body composition of a patient. These methods include 'dual-energy X-ray'-absorptiometry (DEXA) scan, bioelectrica impedance analysis (BIA) and modern imaging techniques including Computed Tomography (CT) and Magnetic Resonance Imaging (MRI). ${ }^{34,35}$ The DEXA scan is typically used to assess bone density in osteoporosis, but can also be used to quantify fat mass and fat-free mass, the latter of which the largest component is skeletal muscle mass. The BIA method is based on the difference in electrical conductance of the different body compartments; muscle mass has a high water content and therefore low electrical resistance, whereas fat mass has a lower water content and higher resistance. Both DEXA and BIA are generally low cost and easy to use, but these are less accurate than modern imaging techniques in quantifying the different tissue compartments, particularly in patients with obesity or during illness. Also, DEXA and $\mathrm{BIA}$ are not routinely retrospectively available or performed in most cancer patients. 
Both CT and MRI allow for the detailed assessment of all body compartments including skeletal muscle mass. In the 1980s, the first studies using MRI and C imaging to investigate body composition were published. In subsequent years, several studies were published describing the assessment of whole body skeletal muscle volume and adipose tissue volume using whole body MRI. In 2004, Shen et al. showed a high correlation between the cross-sectional skeletal muscle area (CSMA) on a single MRI slice at the level of the third lumbar vertebra (L3) and whole body total skeletal muscle volume as measured on whole body MRI. ${ }^{36} \mathrm{~A}$ downside of MRI is that the assessment of skeletal muscle mass generally is very time-consuming and fully manual, whereas the assessment of skeletal muscle mass on CT imaging can be performed using semi-automatic software programs and with predefined Hounsfield unit settings to aid in the identification of skeletal muscle mass. In most studies, Hounsfield unit values between -30 and +150 is accepted as being skeletal muscle mass. ${ }^{37}$ Abdominal $\mathrm{CT}$ is routinely performed during diagnostic work-up and follow-up of many cancer patients, and thus imaging is routinely available for analysis without any extra burden for the patient or healthcare-related costs.

In 2008, Prado et al. showed that there is a linear relationship between a person's height and the CSMA at the level of L3.38 Therefore the CSMA at the level of L3 is adjusted for height, to calculate the lumbar skeletal muscle index (lumbar SMI $\mathrm{cm}^{2} / \mathrm{m}^{2}$ ), as a estimation of a person's total skeletal muscle mass in proportion to stature. ${ }^{38}$ Prado et al were the first to use CT imaging, acquired for routine medical purposes such as diagnosis or treatment response, to investigate the relationship between a low lumbar SMI and adverse outcomes in oncological patients. ${ }^{38,39} \mathrm{~A}$ low lumbar SMI was termed sarcopenia in these studies, although technically only skeletal muscle mass was assessed and not muscle function. The terms sarcopenia and low skeletal muscle mass are often used interchangeably in oncological and surgical literature.

Since 2008, a plethora of retrospective studies have been published that show an association between low skeletal muscle mass as measured at the level of L3 and adverse outcomes. ${ }^{29,30}$ Low skeletal muscle mass has been associated with increased rates of postoperative complications, chemotherapy-related toxicity prolonged hospital stay, increased healthcare related costs, and decreased overall and disease-specific survival rates, in patients with colon cancer, breast cancer, lung cancer, bladder cancer, pancreatic cancer and hematological malignancies, amongst others..$^{29,30,40-46} \mathrm{Abdominal} \mathrm{CT}$ imaging is not routinely performed in head and neck cancer patients, and is often only available in a preselected patient group with advanced disease and high risk features for distant metastasis. This may explain why studies in HNSCC on the association between low skeleta muscle mass and adverse outcomes are relatively scarce. The first study in patients with HNSCC treated with radiotherapy showed that a loss of skeletal muscle mass during treatment was associated with adverse outcomes. ${ }^{47}$ Risk factors for low skeletal muscle mass are often present in patients with HNSCC. Patients with HNSCC are often over the age of 60, particularly those with HPV negative disease. Also, malnutrition is highly common in patients with HNSCC. At diagnosis, up to $50 \%$ of patients with HNSCC present with signs of malnutrition, such as weight loss, a low BMI and/or vitamin deficiencies.

Another application of using routinely performed imaging to investigate the body composition of a patient, is to routinely performed $\mathrm{CT}$ imaging can be used to measure arterial calcification as a biomarker for generalized cardiovascular disease. In breast cancer patients, a higher coronary artery calcium score measured on radiotherapy planning $\mathrm{CT}$ imaging was associated with increased cardiovascular disease risk, and identified patients at increased risk of cardiovascular disease that would otherwise not have been identified. ${ }^{49} \mathrm{In}$ patients in a lung cancer screening program, coronary artery calcium score measured on lung cancer screening CT imaging was associated with increased cardiovascular death. ${ }^{50}$ In esophageal cancer patients undergoing esophagectomy, generalized arterial calcification was associated with anastomotic leakage after surgery. ${ }^{51,52}$

\section{Assessment of body composition on head and neck CT imaging}

In 2016, Swartz et al. published a novel assessment method for skeletal muscle mass using a single CT slice at the level of the third cervical vertebra, which is featured on regular head and neck CT imaging. ${ }^{53} \mathrm{~A}$ good correlation between CSMA at the level of C3 and L3 was found $(r=0.785)$. A multivariate formula to estimate the CSMA at the level of L3 from the CSMA at the level of C3 was formulated; the correlation between the estimated CSMA at the level of $L 3$ and the actual CSMA at the level of $\mathrm{L} 3$ was excellent $(r=0.891)$. This skeletal muscle mass assessment method has not yet been validated.

The measurement method skeletal muscle mass was first applied in a retrospective cohort study by Wendrich et al. ${ }^{54}$ The relationship between low skeletal muscle mass as measured at the level of $\mathrm{C} 3$ and the occurrence of CDLT in patients with locally advanced stage HNSCC treated with CRT with platinum-based chemotherapy was investigated. This study showed that patients with low skeletal muscle mass had a trifold risk of CDLT (44\% versus $14 \%$ ). 


\section{Aims of this thesis}

The radiologically assessed body composition of cancer patients has been of research interest in recent years. Specifically a low skeletal muscle mass has been associated with increased postoperative complications, prolonged hospital stay and decreased survival in surgical and medical oncology. In head and neck cancer, the predictive and prognostic value of low skeletal muscle mass has not yet been researched as thoroughly as in most types of cancer. The most common measurement method for skeletal muscle mass, using routinely performed abdominal CT imaging at the level of the third lumbar vertebra is not applicable in head and neck cancer patients. The recently published measurement method for skeletal muscle mass at the level of the third cervical vertebra is applicable in almost all head and neck cancer patients, because imaging of the head and neck area is almost always available.

The aims of this thesis are trifold: 1. the validation of skeletal muscle mas measurement at the level of $\mathrm{C} 3$ in Part II of this thesis; 2. to investigate the clinical relevance of low skeletal muscle mass, sarcopenia and arterial calcifications, as measured on routinely performed head and neck CT or MRI, on treatment outcome in Part III of this thesis; and 3. to investigate the mechanism behind the association between low skeletal muscle mass and increased incidence of CDLT in Part IV of this thesis.

In Part II of this thesis, the measurement of skeletal muscle mass at the level o C3 is investigated in terms of accuracy, interobserver variability and relationship with whole body MRI measurements. In Chapter $\mathbf{2}$ of this thesis, the relationship between skeletal muscle mass at the level of C3 and at the level of L3 is reexamined, in order to validate this new measurement method against the generally used L3 measurement. In Chapter 3, the interobserver agreement of skeletal muscle mass measurement at the level of C3 is examined. Chapter 4 aims to describe the relationship between skeletal muscle mass at the level of $\mathrm{C} 3$ and whole body skeletal muscle volume as measured on whole body MRI, and validate a measurement of skeletal muscle mass at the level of $\mathrm{C} 3$ against this gold standard.

In Part III of this thesis the clinical relevance of pre-treatment body composition in head and neck cancer patients as measured on routinely performed imaging of the head and neck area is defined. In Chapter 5, the relationship between low skeletal muscle mass and chemotherapy dose-limiting toxicity is investigated in a cohort of patients with locally advanced HNSCC treated with primary CRT with high dose cisplatin. Chapter 6 will investigate the relationship between low skeletal muscle mass and short and long term outcomes in patients undergoing total laryngectomy. In Chapter 7 , the presence of sarcopenia, defined as a low skeletal muscle mass and low muscle function, is researched in a cohort of elderly head and neck cancer patients, and its relationship with survival is investigated.

Chapter 8 describes the relationship between low skeletal muscle mass and overall survival in patients with oropharyngeal cancer. Chapter 9 explores the incidence of arterial calcifications on CT imaging of the head and neck area in patients undergoing total laryngectomy, and examines its association with the occurrence of a pharyngocutaneous fistula.

Finally, in Part IV, the mechanism behind the association between low skeletal muscle mass and increased incidence of CDLT is explored. A hypothesis for this phenomenon is that the pharmacokinetics of hydrophilic chemotherapeutical agents including cisplatin is altered with respect to the distributional volume in patients with an abnormal body composition; namely, that patients with low skeletal muscle mass and normal or high adipose tissue mass may actually receive a relatively high dose of the chemotherapeutical agents due to its main distribution into the fat-free body mass, of which skeletal muscle mass is the argest contributor. In Chapter 10, the study protocol for prospective study investigating the relationship between low skeletal muscle mass, body surface area and cisplatin pharmacokinetics is described. The results of this study will provide clarity into the relationship between low skeletal muscle mass, cisplatin pharmacokinetics and cisplatin toxicity.

This thesis describes the relationship between radiologically assessed body composition and clinical outcomes in head and neck cancer patients, and may allow for personalized medicine in terms of treatment adaptation and ris optimization with regard to the individual patient's body composition, in a patient population known to be at high risk of adverse clinical outcomes. 


\section{References} Bril SI, Pothen AJ, Bree R. Sarcopenie bij patiënten met hoofd-halskanker. Ned Tijdschr Oncol.
2017;2:7.

2. Bray F, Ferlay J, Soerjomataram I, Siegel RL, Torre LA, Jemal A. Global cancer statistics 2018 GLOBOCAN estimates of incidence and mortality worldwide for 36 cancers in 185 countries. CA Cancer J Clin. 2018;68(6):394-424.

3. KWF. No Title. Cijfers over kanker. https://iknl.nl/nkr-cijfers

4. Argiris A, Karamouzis MV., Raben D, Ferris RL. Head and neckcancer.Lancet.2008;371(9625):16951709 .

5. Kobayashi K, Hisamatsu K, Suzui N, Hara A, Tomita H, Miyazaki T. A Review of HPV-Related Head and Neck Cancer. J Clin Med. 2018;7(9):241.

6. Marur S, D'Souza G, Westra WH, Forastiere AA. HPV-associated head and neck cancer: a virusrelated cancer epidemic. Lancet Oncol. 2010;11(8):781-789.

7. Grégoire V, Lefebvre J-L, Licitra L, Felip E. Squamous cell carcinoma of the head and neck: EHNS-ESMO-ESTRO Clinical Practice Guidelines for diagnosis, treatment and follow-up. An Oncol. 2010;21:v184-v186.

8. Lacas B, Carmel A, Landaus C, et al. Meta-analysis of chemotherapy in head and neck cancer (MACH-NC): An update on 107 randomized trials and 19,805 patients, on behalf of $\mathrm{MACH}-\mathrm{NC}$ Group. Radiother Oncol. 2021;156(3): 281-293.

9. Forastiere AA, Zhang Q, Weber RS, et al. Long-Term Results of RTOG 91-11: A Comparison of Three Nonsurgical Treatment Strategies to Preserve the Larynx in Patients With Locally Advanced Larynx Cancer. J Clin Oncol. 2013;31(7):845-852.

10. Vermorken J, Specenier P. Cetuximab: its unique place in head and neck cancer treatment. Biologics. 2013;7:77-90.

11. Bonner JA, Harari PM, Giralt J, et al. Radiotherapy plus cetuximab for locoregionally advanced head and neck cancer: 5-year survival data from a phase 3 randomised trial, and relation between cetuximab-induced rash and survival. Lancet Oncol. 2010 Jan;11(1):21-8.

12. Szturz P, Wouters K, Kiyota N, et al. Low-Dose vs. High-Dose Cisplatin: Lessons Learned From 59 Chemoradiotherapy Trials in Head and Neck Cancer. Front Oncol. 2019 Feb 21;9:86.

13. Sztutz P, Wouters K, Kiyota N, et al. Weekly Low-Dose Versus Three-Weekly High-Dose Cisplatin for Concurrent Chemoradiation in Locoregionally Advanced Non-Nasopharyngeal Head and Neck Cancer: A Systematic Review and Meta-Analysis of Aggregate Data. Oncologist. 2017;22(9):1056-1066

14. Mehanna H, Robinson M, Hartley A, et al. Radiotherapy plus cisplatin or cetuximab in lowrisk human papillomavirus-positive oropharyngeal cancer (De-ESCALaTE HPV): an open-label randomised controlled phase 3 trial. Lancet. 2019;393(10166):51-60.

15. Gillison ML, Trotti AM, Harris J, et al. Radiotherapy plus cetuximab or cisplatin in human papillomavirus-positive oropharyngeal cancer (NRG Oncology RTOG 1016): a randomised, multicentre, non-inferiority trial. Lancet. 2019:393(10166):40-50

16. Szturz P, Cristina V, Herrera Gómez RG, et al. Cisplatin Eligibility Issues and Alternative Regimen in Locoregionally Advanced Head and Neck Cancer: Recommendations for Clinical Practice. Front Oncol. 2019. 11;9:464

17. Strojan $\mathrm{P}$, Vermorken $\mathrm{JB}$, Beitler JJ, et al. Cumulative cisplatin dose in concurren chemoradiotherapy for head and neck cancer: A systematic review. Eisele DW, ed. Head Neck. 2016;38(S1):E2151-E2158

18. Spreafico $A$, Huang $S H, X u W$, et al. Impact of cisplatin dose intensity on human papillomavirusrelated and -unrelated locally advanced head and neck squamous cell carcinoma. Eur J Cancer. 2016;67:174-182.

19. Ang K, Zhang Q, Wheeler RH, et al. A phase III trial (RTOG 0129) of two radiation-cisplatin regimens for head and neck carcinomas (HNC): Impact of radiation and cisplatin intensity on outcome. J Clin Oncol. 2010;28(15_suppl):5507-5507.

20. Forastiere AA, Ismaila N, Lewin JS, et al. Use of Larynx-Preservation Strategies in the Treatment of Laryngeal Cancer: American Society of Clinical Oncology Clinical Practice Guideline Update. Clin Oncol. 2018;36(11):1143-1169.
21. Sanabria A, Chaves ALF, Kowalski LP, et al. Organ preservation with chemoradiation in advanced laryngeal cancer: The problem of generalizing results from randomized controlled trials. Auris

22. Shodo R, Yamazaki K, Ueki Y, Takahashi T, Horii A. Sarcopenia predicts a poor treatment outcome in patients with head and neck squamous cell carcinoma receiving concurren chemoradiotherapy. Eur Arch Otorhinolaryngol. 2020:12(3):584.

23. Hasan Z, Dwivedi RC, Gunaratne DA, Virk SA, Palme CE, Riffat F. Systematic review and metaanalysis of the complications of salvage total laryngectomy. Eur J Surg Oncol. 2017;43(1):42-51

24. Goepfert RP, Hutcheson KA, Lewin JS, et al. Complications, hospital length of stay, and readmission after total laryngectomy. Cancer. 2017;123(10):1760-1767.

25. Paydarfar JA, Birkmeyer NJ. Complications in Head and Neck Surgery. Arch Otolaryngol Neck Surg. 2006;132(1):67.

26. Lansaat $\mathrm{L}$, van der Noort $\mathrm{V}$, Bernard $\mathrm{SE}$, et al. Predictive factors for pharyngocutaneous fistulization after total laryngectomy: a Dutch Head and Neck Society audit. Eur Arch OtoRhino-Laryngology. 2018;275(3):783-794.

27. Paleri V, Drinnan M, van den Brekel MWM, et al. Vascularized tissue to reduce fistula following salvage total laryngectomy: A systematic review. Laryngoscope. 2014;124(8):1848-1853.

28. Prado CM, Cushen SJ, Orsso CE, Ryan AM. Sarcopenia and cachexia in the era of obesity: clinica and nutritional impact. Proc Nutr Soc. 2016;75(2):188-198.

9. Rier HN, Jager A, Sleijfer S, Maier AB, Levin M. The Prevalence and Prognostic Value of Low

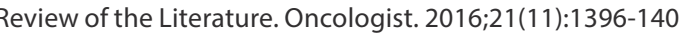

0. Levolger S, van Vugt JLA, de Bruin RWF, IJzermans JNM. Systematic review of sarcopenia in patients operated on for gastrointestinal and hepatopancreatobiliary malignancies. $\mathrm{Br} J$ Surg. 2015;102(12):1448-1458.

31. Jones K, Gordon-Weeks A, Coleman C, Silva M. Radiologically Determined Sarcopenia Predicts Morbidity and Mortality Following Abdominal Surgery: A Systematic Review and MetaAnalysis. World J Surg. 2017;41(9):2266-2279.

32. Cruz-Jentoft AJ, Baeyens JP, Bauer JM, et al. Sarcopenia: European consensus on definition an diagnosis: Report of the European Working Group on Sarcopenia in Older People. Age Ageing. 2010;39(4):412-423.

33. von Haehling S, Morley JE, Anker SD. An overview of sarcopenia. facts and numbers on prevalence and clinical impact. J Cachexia Sarcopenia Muscle. 2010;1 (2):129-133.

34. Prado CMM, Heymsfield SB. Lean Tissue Imaging. J Parenter Enter Nutr. 2014;38(8):940-953.

35. Lee SY, Gallagher D. Assessment methods in human body composition. Curr Opin Clin Nutr Metab Care. 2008;11(5):566-572.

36. Shen W, Punyanitya M, Wang Z, et al. Total body skeletal muscle and adipose tissue volumes: estimation from a single abdominal cross-sectional image. J Appl Physiol. 2004;97(6):23332338.

37. B. Heymsfield S, Wang Z, Baumgartner RN, Ross R. Human Body Composition: Advances in Models and Methods. Annu Rev Nutr. 1997;17(1):527-558,

38. Prado CM, Lieffers JR, McCargar $\mathrm{L}$, et al. Prevalence and clinical implications of sarcopenic obesity in patients with solid tumours of the respiratory and gastrointestinal tracts: population-based study. Lancet Oncol. 2008;9(7):629-635.

39. Prado CMM, Baracos VE, McCargar LJ, et al. Sarcopenia as a Determinant of Chemotherapy Toxicity and Time to Tumor Progression in Metastatic Breast Cancer Patients Receiving Capecitabine Treatment. Clin Cancer Res. 2009;15(8):2920-2926.

40. van Vugt JLA, Buettner S, Levolger S, et al. Low skeletal muscle mass is associated with increased hospital expenditure in patients undergoing cancer surgery of the alimentary tract. Bianchi $C$, ed. PLoS One. 2017;12(10):e0186547.

41. van Dijk DPJ, Bakens MJAM, Coolsen MME, et al. Low skeletal muscle radiation attenuation and visceral adiposity are associated with overall survival and surgical site infections in patients with pancreatic cancer. J Cachexia Sarcopenia Muscle. 2017:8(2):317-326. 
42. Martin L, Hopkins J, Malietzis G, et al. Assessment of Computed Tomography (CT)-Defined Muscle and Adipose Tissue Features in Relation to Short-Term Outcomes After Elective Surgery for Colorectal Cancer: A Multicenter Approach. Ann Surg Oncol. 2018;25(9):2669-2680.

43. Choi MH, Oh SN, Lee IK, Oh ST, Won DD. Sarcopenia is negatively associated with long-term outcomes in locally advanced rectal cancer. J Cachexia Sarcopenia Muscle. 2018;9(1):53-59.

44. Srdic D, Plestina S, Sverko-Peternac A, Nikolac N, Simundic A-M, Samarzija M. Cancer cachexia, sarcopenia and biochemical markers in patients with advanced non-small cell lung cancer-

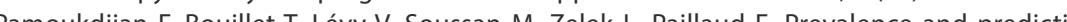

45. Pamoukdjian $F$, Boullet $T$, Lévy $V$, Soussan $M$, Zelek L, Paillaud E. Prevalence and predictive value of pre-therape

46. Nakamura N, Hara T, Shibata $Y$, et al. Sarcopenia is an independent prognostic factor in male patients with diffuse large B-cell lymphoma. Ann Hematol. 2015;94(12):2043-2053.

47. Grossberg AJ, Chamchod S, Fuller CD, et al. Association of Body Composition With Survival and Locoregional Control of Radiotherapy-Treated Head and Neck Squamous Cell Carcinoma. JAMA Oncol. 2016,2(6):782.

48. Hébuterne X, Lemarié E, Michallet M, de Montreuil CB, Schneider SM, Goldwasser F. Prevalence of Malnutrition and Current Use of Nutrition Support in Patients With Cancer. J Parenter Enter

49. Gernaat SAM, Iśgum I, de Vos BD, et al. Automatic Coronary Artery Calcium Scoring on Radiotherapy Planning CT Scans of Breast Cancer Patients: Reproducibility and Associatio with Traditional Cardiovascular Risk Factors. Zirlik A, ed. PLoS One. 2016;11(12):e0167925.

50. Garg PK, Jorgensen NW, McClelland RL, et al. Use of coronary artery calcium testing to improve coronary heart disease risk assessment in a lung cancer screening population: The Multi-Ethni Study of Atherosclerosis (MESA). J Cardiovasc Comput Tomogr. Nov-Dec 2018;12(6):493-499.

1. Borggreve AS, Goense L, van Rossum PSN, van Hillegersberg R, de Jong PA, Ruurda JP. Generalized cardiovascular disease on a preoperative CT scan is predictive for anastomotic leakage after esophagectomy. Eur J Surg Oncol. 2018;44(5):587-593.

52. Goense L, van Rossum PSN, Weijs TJ, et al. Aortic Calcification Increases the Risk of Anastomotic Leakage After Ivor-Lewis Esophagectomy. Ann Thorac Surg. 2016;102(1):247-252.

53. Swartz JE, Pothen AJ, Wegner I, et al. Feasibility of using head and neck CT imaging to assess skeletal muscle mass in head and neck cancer patients. Oral Oncol. 2016;62:28-33.

54. Wendrich AW, Swartz JE, Bril Sl, et al. Low skeletal muscle mass is a predictive factor for chemotherapy dose-limiting toxicity in patients with locally advanced head and neck cancer. Oral Oncol. 2017;71:26-33. 


\section{Part II}

The assessment of low skeletal muscle mass in head and neck cancer patients 


\section{Chapter 2}

Validation of skeletal muscle mass assessment at the level of the third cervical vertebra in patients with head and neck cancer

Sandra I. Bril*, Najiba Chargi", Anne W. Wendrich, Inge Wegner, Gijs H. Bol, Ernst J.

Smid, Pim A. de Jong, Lot A. Devriese and Remco de Bree

*contributed equally

Submitted 


\section{Abstract}

Background Low skeletal muscle mass (SMM) is associated with adverse outcomes. SMM is often assessed at the third lumbar vertebra (L3) on abdominal imaging. Abdominal imaging is not routinely performed in patients with head and neck cancer (HNC). We aim to validate SMM measurement at the level of the third cervical vertebra (C3) on head and neck imaging.

Material and methods Patients with pre-treatment whole-body computed tomography (CT) between 2010 and 2018 were included. Cross-sectional muscle area (CSMA) was manually delineated at the level of C3 and L3. Correlation coefficients and intraclass correlation coefficients (ICCS) were calculated. Cohen's kappa was used to assess the reliability of identifying a patient with low SMM.

Results Two hundred patients were included. Correlation between CSMA at the level of C3 and L3 was good $(r=0.75, \mathrm{p}<0.01)$. Using a multivariate formula to estimate CSMA at L3, including gender, age, and weight, correlation improved ( $r=$ $0.82, p<0.01)$. The agreement between estimated and actual CSMA at L3 was good (ICC $0.78, p<0.01)$. There was moderate agreement in the identification of patients with low SMM based on the estimated lumbar skeletal muscle mass index (LSMI) and actual LSMI (Cohen's $\kappa: 0.57,95 \% \mathrm{Cl}$ 0.45-0.69).

Conclusions CSMA at C3 correlates well with CSMA at L3. There is moderate agreement in the identification of patients with low SMM based on the estimated LSMI (using a measurement at C3) and actual LSMI.

\section{Introduction}

Over the last decade, research into the specific body composition of cancer patients and its relationship with clinical outcomes has tremendously increased due to the use of diagnostically performed imaging for quantification of different body compartments, including skeletal muscle mass (SMM) and adipose tissue mass. 1,2 Specifically a state of low SMM, sometimes termed sarcopenia, has gained interest as a novel risk factor for negative short- and long-term outcomes. In breast, gastro-intestinal, hepato-pancreatic-biliary and respiratory cancer amongst others, low SMM is associated with increased incidence of postoperative complications, chemotherapy-related toxicity, prolonged hospital stay and shorter disease-free and overall survival., ${ }^{3,4}$

SMM is most commonly assessed on a single CT slice at the level of the third umbar vertebra (L3), which has shown to have excellent correlation with whole body skeletal muscle volumes as measured using whole body MRI.5,6 The crosssectional skeletal muscle area (CSMA) at the level of L3 is then most commonly normalized for stature, to calculate the lumbar skeletal muscle index (lumba SMI). ${ }^{5}$ The lumbar SMI is used as a proxy for SMM as a whole, and several cut-offs have been published to identify patients with low SMM. ${ }^{4}$

In head and neck cancer (HNC), abdominal CT imaging is not commonly performed as part of the routine diagnostic work-up. Therefore, abdominal CT imaging to quantify SMM is not routinely applicable in HNC patients. To overcome this, a measurement method for SMM at the level of the third cervical vertebra (C3), which is featured on standard CT imaging of the head and neck area, was published by Swartz et al. ${ }^{7}$ A multivariate formula to calculate CSMA at the leve of $L 3$ from CSMA at the level of C3 was also published, to allow for comparison to other oncological research. ${ }^{7}$ Wendrich et al. published a cut-off value for low SMM in HNC patients based on this method. ${ }^{8}$

The measurement method for SMM at the level of C3 was used in several studies in HNC patients. The incidence of low SMM was high in several studies; typically 50\% of patients and sometimes up to $77 \%$ of patients had low SMM prior to start of treatment. ${ }^{8-11}$ In HNC patients, low SMM was associated with negative short- and long-term outcome such as chemotherapy dose-limiting toxicity, postoperative complications and decreased survival. ${ }^{8,9,12,13}$ Only one previous study by Ufuk et al. has investigated the correlation between CSMA measurement at the level of C2, C3, C4 and L3. They showed that CSMA at the level of C3 was best associated 
with CSMA at the level of L3, and that the correlation between CSMA at the leve of C3 and CSMA at the level of L3 was excellent. ${ }^{14}$ Ufuk et al. segmented the sternocleidomastoids (SCM) and paravertebral muscles (PVM) separately, Swartz et al. recommends using the CSMA at C3 of both the SCM and PVM. Ufuk et al. also used cut-off values for low SMM based on the study of Prado et al. which did not include HNC patients and did not validate the formula proposed by Swartz et al.

Our current study aimed to validate the association between skeletal muscle area at the level of C3 and the level of L3 in a larger cohort of treatment-naïve HNC patients. It also aimed to investigate the accuracy of identifying patients with low SMM using the previously published cut-off value.

\section{Patients and Methods}

\section{Ethical considerations}

The design of this study was approved by the Medical Ethical Research Committee of the University Medical Center Utrecht (approval ID 16/595 C). All data was retrieved retrospectively and processed in an anonymized fashion.

\section{Study population}

Patients who were diagnosed at the University Medical Center Utrecht, The Netherlands between 2010 and 2018 with a primary head and neck squamous cell carcinoma were evaluated for this study. Since the effect of previous treatments of the neck on CSMA measurement at the level of C3 is not known, patients previously treated with surgery or radiotherapy of the neck were excluded. Patients were included if a pre-treatment whole body FDG-PET/CT scan in radiation mould (as part of radiotherapy treatment planning) was available. Othe relevant parameters, including length and weight at the time of imaging, sex, age tumor localization and clinical TNM stage $\left(7^{\text {th }}\right.$ and $8^{\text {th }}$ edition) were retrospectively retrieved. In total, 200 patients were selected.

\section{Assessment of cross-sectional muscle area}

Pre-treatment FDG-PET/CT-imaging was performed in all patients according to a standardized protocol. Muscle tissue was identified using Hounsfield Unit (HU) range settings from -29 to $+150 \mathrm{HU}$, which is specific for muscle tissue. Muscle tissue was delineated at the level of the third lumbar vertebra (L3) and the third cervical vertebra (C3). The CSMA was defined as the pixel area within the delineated area with a radiodensity between -29 and +150 HU. ${ }^{15,16}$ Delineation of muscle tissue was manually performed using the Volumetool v.1.6.5 Research Software Package, designed in our center as an image evaluation, registration and delineation system for radiotherapy planning. ${ }^{17}$

For delineation of muscle tissue at the level of $L 3$, the first slide when scrolling from caudal to cranial direction to show the entire vertebral arc and both transverse processes was selected. The contours of the abdominal wall and paraspinal muscles were manually traced. CSMA at the level of $L 3$ was calculated by adding up the abdominal wall and paraspinal muscle area. For delineation of muscle tissue at the level of $\mathrm{C} 3$, the first slide when scrolling from caudal to crania direction to show both transverse processes and the entire vertebral arc was selected. The contours of the paravertebral muscles and both sternocleidomastoid muscles were manually traced. The CSMA at the level of C3 was calculated as the sum of the paravertebral muscle and both sternocleidomastoid muscles. If eviden ymph node metastasis hindered accurate delineation of one sternocleidomastoid muscle, the CSMA of the contralateral sternocleidomastoid muscle was used as an estimation of the CSMA of the affected sternocleidomastoid muscle. ${ }^{7}$ After delineation, CSMA was automatically retrieved from Volumetool. First, all head and neck CT scans (C3) were delineated, and afterwards all abdominal scans (L3). Figure 1 shows muscle tissue delineation at the level of $\mathrm{C} 3$ and $\mathrm{L} 3$.

\section{Cross-sectional muscle area at the level of L3}

As well as the actually measured CSMA at the level of L3, the CSMA at the level of 3 was also estimated from the CSMA at the level of C3 using the prediction rule as described by Swartz et al, see Formula 1. ${ }^{7}$ The lumbar SMI was then calculated using the formula published by Prado et al, see Formula 2. ${ }^{5}$

Formula 1: CSMA at L3 $\left(\mathrm{cm}^{2}\right)=27.304+1.363 *$ CSMA at C3 $\left(\mathrm{cm}^{2}\right)+0.640 *$ Weight $(\mathrm{kg})+26.442 *$ Gender (Gender $=1$ for female, 2 for male) $-0.671 *$ Age (years)

Formula 2: $\mathrm{SMI}\left(\mathrm{cm}^{2} / \mathrm{m}^{2}\right)=\mathrm{CMSA}$ at L3 $/\left(\right.$ height $\left.^{2}\right)$

Low SMM was defined as a lumbar $\mathrm{SMI}<43.2 \mathrm{~cm}^{2} / \mathrm{m}^{2}$, as previously published by Wendrich et al. ${ }^{8}$

\section{Statistical analysis}

All statistical analyses were performed using the IBM SPSS Statistics version 25.0 software package (Chicago, Illinois, USA). There were no missing data. A test for normality (Shapiro-Wilk test) was performed to assess whether continuous 
variables were normally distributed. For table 1. continuous data are represented as mean \pm standard deviation (SD) if normally distributed, and median \pm range if skewed. Categorical data are represented as a number and percentage of total. The student's $t$-test, one-way ANOVA, Mann-Whitney $U$ test were used where appropriate. Depending on normality of variables, Pearson or Spearman Rank correlation coefficients were calculated to assess correlation between CSMA at the level of C3, at the level of L3 and predicted CSMA at the level of L3.

To assess the agreement between measurements, we calculated intraclass correlation coefficients (ICCs) using a two-way mixed single measures model with absolute agreement. The ICCs were rated as poor ( $0.00-0.49)$, fair to good $(0.50$ - 0.74), good (0.75-0.90) and excellent $(>0.90) .{ }^{18}$ For agreement in classification of patients with low SMM, Cohen's $k$ was used. The agreement was rated as no agreement $(<0)$, slight $(0.01-0.20)$, fair $(0.21-0.40)$, moderate $(0.41-0.60)$, substantial $(0.61-0.80)$ and almost perfect $(0.81-1.00) .{ }^{19} \mathrm{~A}$ two-tailed test of significance $(p=0.05)$ was used.

\section{Figure 1. example of skeletal muscle tissue delineation}

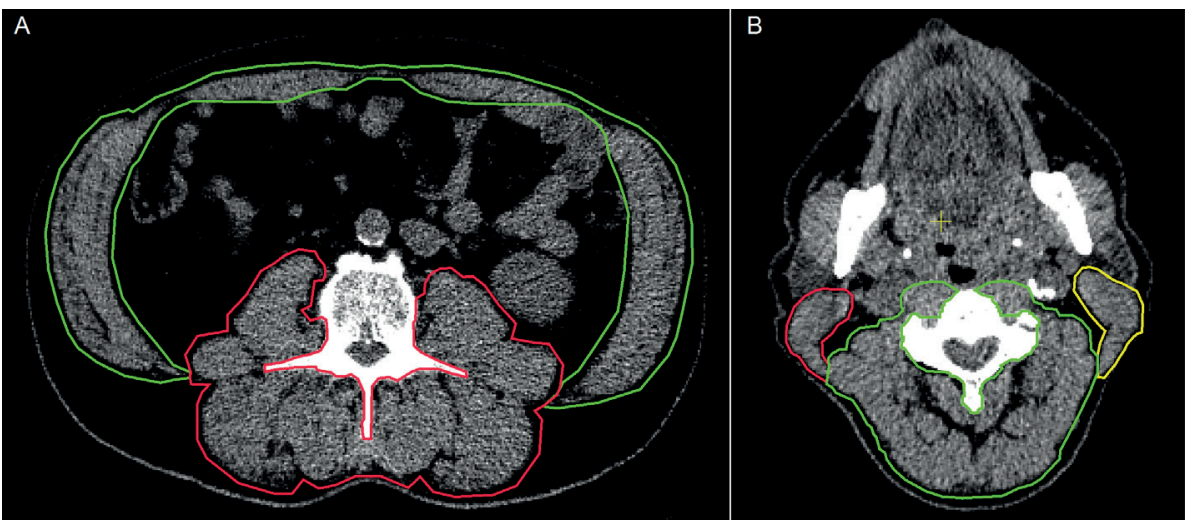

Figure 1: delineation of skeletal muscle tissue on transversal CT imaging at the level of L3 (1a) and at the level of C3 (1b). A Hounsfield Unit window of -29 to +150 was used to accentuate skeletal muscle

\section{Results}

\section{Patient characteristics}

For this study, 200 patients were included for analyses. Baseline patient characteristics are shown in Table 1. Patients were predominantly male and often presented with advanced disease (T3-4; N+). Weight and BMI at diagnosis were normally distributed. On average, patients had a normal BMI.

\begin{tabular}{lc} 
Table 1: Patient characteristics & \\
\hline Characteristic & $\mathbf{n}$ (\% or SD) total $\mathbf{n}=\mathbf{2 0 0}$ \\
\hline Gender & \\
Male & $147(73.5)$ \\
Female & $53(26.5)$ \\
Age at diagnosis (years) & \\
Mean (SD) & $63.5(8.3)$ \\
Range & $44.9-85.6$ \\
Weight at diagnosis (kg) & \\
Mean (SD) & $74.1(16.4)$ \\
Range & $40.0-122.0$ \\
BMI (weight/height') & \\
Mean (SD) & $24.2(4.6)$ \\
Range & $14.0-40.0$ \\
Localization & \\
Hypopharynx & $57(28.5)$ \\
Larynx & $40(20.0)$ \\
Oropharynx & $83(41.5)$ \\
Oral cavity & $12(6.0)$ \\
Nasopharynx & $5(2.5)$ \\
Unknown primary & $3(1.5)$ \\
T-status & \\
T1-2 & $92(46.0)$ \\
T3-4 & $108(54.0)$ \\
N-status & $73(36.5)$ \\
N0 & $61(30.5)$ \\
N1-2a & $66(33.0)$ \\
N2b-3b & \\
M-status & $183(91.5)$ \\
M0 & $10(5.0)$ \\
M+ & $7(3.5)$ \\
Mx & \\
\hline &
\end{tabular}

\section{Image analysis}

Delineation of muscle tissue at the level of C 3 was successful in all patients. Six patients $(8.6 \%)$ had evident growth of a lymph node metastasis into the SCM muscles. In these 6 patients, the CSMA of the affected SCM muscle was substituted by the CSMA of the unaffected, contralateral SCM muscle. 


\section{Correlation between CSMA at $\mathrm{C} 3$ and $\mathrm{L} 3$}

Skeletal muscle area at the level of C3 was not normally distributed (ShapiroWilk test $<0.05$ ). Spearman rank correlation analysis showed a good correlation between CSMA at C3 and CSMA at L3 (Spearman's $r_{s}=0.75 ; p<0.01$ ). Figure 2 shows the direct correlation between CSMA measurements at the level of C3 and L3. Correlation between CSMA at C3 and CSMA at L3 was higher than the correlation between cross-sectional area of the paravertebral muscles only at C3 and CSMA at L3 (Spearman's $r_{s}=0.75$ versus $r_{s}=0.70$ ).

\section{Figure 2: correlation between CSMA at the level of C3 and (actual) CSMA at the level of}

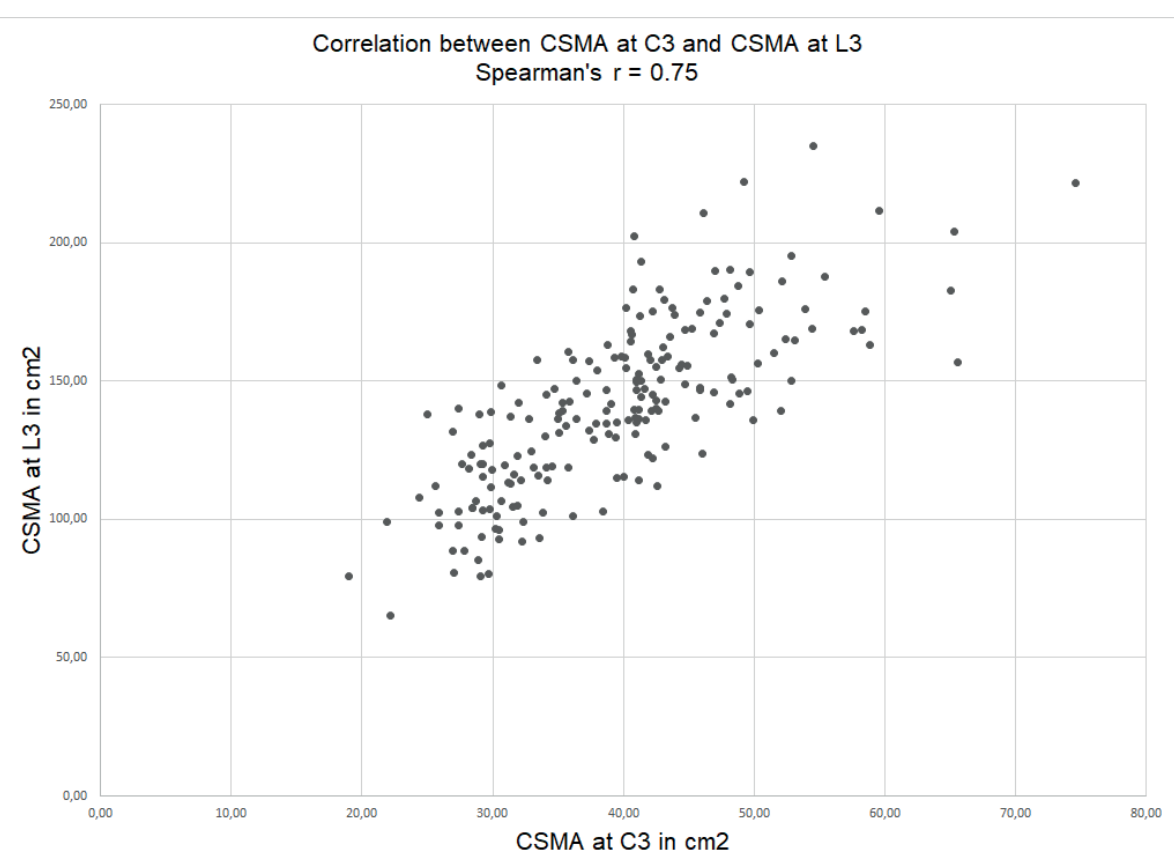

CSMA at L3 was estimated from CSMA at C3 using the multivariate formula as described earlier (Formula 1). Actual CSMA at L3 and estimated CSMA at L3 were normally distributed (Shapiro-Wilk test: $p>0.05$ ). Figure 3 shows the correlation between the estimated CSMA at L3 and the actual CSMA at L3. Pearson correlation analysis showed a high correlation between the estimated CSMA at L3 and the actual CSMA at L3 $(r=0.82 ; \mathrm{p}<0.01)$. The mean difference between the estimated CSMA at L3 and the actual CSMA at L3 was calculated (mean $-9.4 \mathrm{~cm}^{2}$, SD $17.8 \mathrm{~cm}^{2}$ $7.0 \%$ of total CSMA at L3), meaning that the estimated CSMA at L3 was lower than the actual CSMA at L3. In 13 of 200 patients (7\%) the estimated and actual CSMA at $L 3$ differed more than 1.96 standard deviation from the average, suggesting a reasonably good agreement. The ICC between estimated CSMA at L3 and actua CSMA at L3 was good: 0.78 (95\% Cl: $0.61-0.86, \mathrm{p}<0.01)$.

Figure 3: correlation between estimated CSMA at the level of $L 3$ and actual CSMA at the level of $L 3$

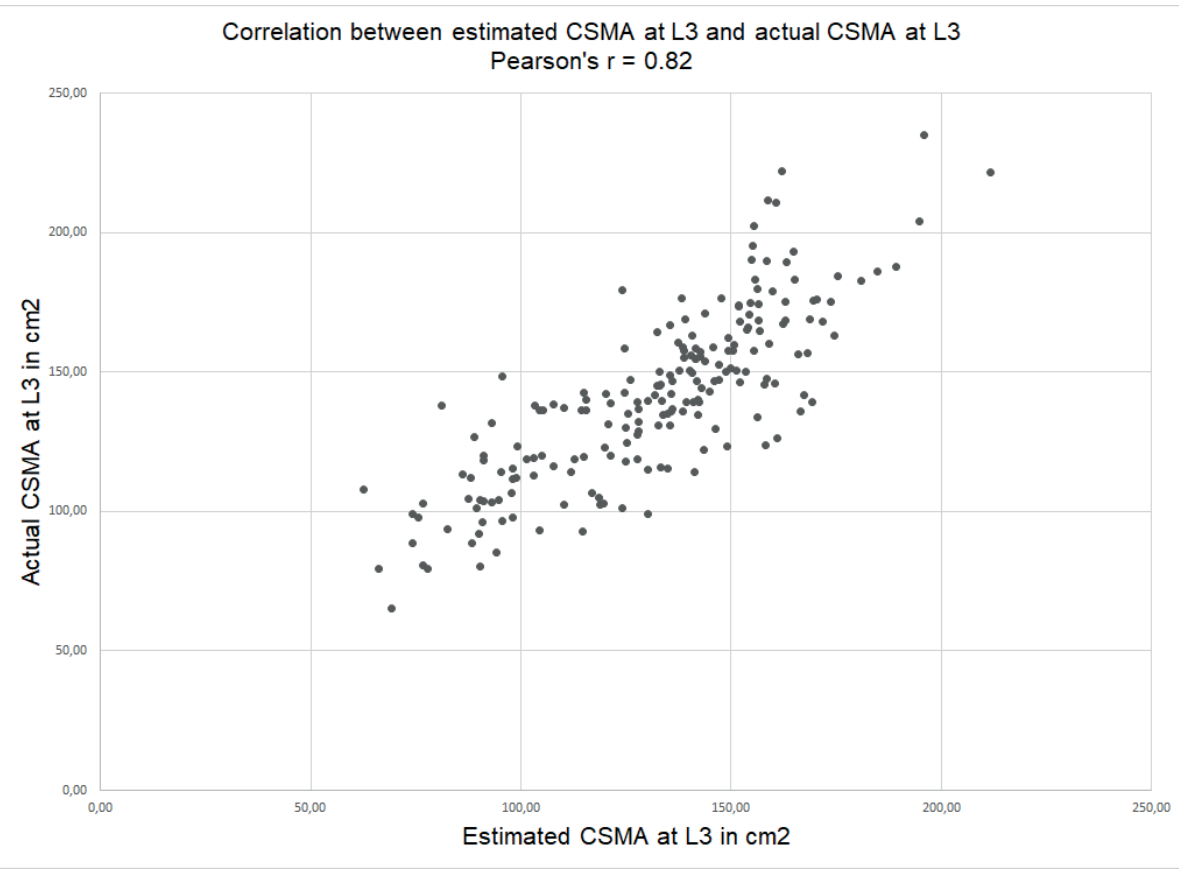

Agreement and accuracy in identification of patients with low skeletal muscle mass

Using Formula 2, the estimated lumbar SMI and actual lumbar SMI were calculated. The previously published cut-off value of $\leq 43.2 \mathrm{~cm} / \mathrm{m}^{2}$ was used to determine low SMM. Using this cut-off, 96 patients were determined to have low SMM using the estimated lumbar SMI, and 77 patients had low SMM using the actual lumbar SMl; see Table 2. The sensitivity of identifying patients with low SMM using the estimated lumbar SMI and a cut-off of $\leq 43.2 \mathrm{~cm} / \mathrm{m}^{2}$ was $84.4 \%$ and the specificity was $74.8 \%$. The positive predictive value of the estimated lumbar SMI was $67.7 \%$ and the negative predictive value was $88.5 \%$. The false positive value, indicating the number of patients that incorrectly were identified as having 
low SMM, was $25.2 \%$. Cohen's kappa for agreement between low SMM using the estimated and the actual lumbar SMI was 0.57 , indicating moderate agreement.

Table 2: Agreement between estimated and actual low skeletal muscle mass, defined as a lumbar $\mathrm{SMI} \leq 43.2 \mathrm{~cm}^{2} / \mathrm{m}^{2}$

\begin{tabular}{|c|c|c|c|c|c|}
\hline & & \multicolumn{2}{|c|}{$\begin{array}{l}\text { Low skeletal muscle mass: } \\
\text { actual lumbar } S M I \leq 43.2 \mathrm{~cm}^{2} / \mathrm{m}^{2}\end{array}$} & \multirow[t]{2}{*}{ Sum } & \\
\hline & & Yes & No & & \\
\hline \multirow{4}{*}{$\begin{array}{l}\text { Low skeletal muscle mass: } \\
\text { estimated lumbar SMI } \leq 43.2 \\
\text { sum }\end{array}$} & Yes & 65 & 31 & 96 & $P P V=68 \%$ \\
\hline & No & 12 & 92 & 104 & $\mathrm{NPV}=88 \%$ \\
\hline & & 77 & 123 & 200 & \\
\hline & & Sens $84 \%$ & Spec $75 \%$ & & \\
\hline
\end{tabular}

Sens = sensitivity; spec $=$ specificity; $P P V=$ positive predictive value; NPV = negative predictive value

\section{Discussion}

There is a need for a robust, easy and widely available SMM quantification too specifically for HNC patients, to allow for routine assessment of SMM without the need for additional diagnostics. Swartz et al proposed a measurement of CSMA at the level of C3 as an alternative to measurement of CSMA at the level of L3, using standard head and neck CT imaging. Our current study shows that measurement of CSMA at the level of C3 provides a good estimation of CSMA at the level of $L 3$ $\left(r_{s}=0.75\right)$. Total CSMA at the level of C 3 had a higher correlation with CSMA at the level of L3 than cross-sectional area of paravertebral muscles only $(r=0.75$ versus $r_{s}=0.70$ ), which is in agreement with results of a previous study, albeit slightly lower. ${ }^{14}$ Using the same multivariate formula as described earlier, in a different set of patients, we found a very good correlation $(r=0.82)$ between CSMA at the level of C3 and L3. The agreement in identification of patients with low SMM was moderate and the probability that a patient with low SMM according to C3 has a low SMM with the LC method is 68\%. A measurement of CSMA at the level of C3 provides a good estimation of CSMA at the level of $\mathrm{L} 3$ and subsequent analysis without the need for additional testing. Interobserver agreement was not further tested in this study; a previous study showed excellent interobserver agreement for CSMA measurement at the level of $\mathrm{C} 3 .{ }^{20}$

There was some variation in the identification of patients with low SMM based on the estimated lumbar SMI compared to the actual lumbar SMI. The estimated lumbar SMI however was on average $-9.4 \mathrm{~cm}^{2}$ lower than the actual lumbar SMI; classifying more patients as having low SMM than there actually are. Because the cut-off value for low SMM (lumbar SMI $\leq 43.2 \mathrm{~cm}^{2} / \mathrm{m}^{2}$ ) is based on estimated lumbar SMI by use of segmented CSMA at the level of C3, other cut-off values for lumbar SMI may apply when segmentation of CSMA at the level of L3 is performed directly. This may explain the false positive rate of $25.2 \%$. However, we acknowledge that an estimation of CSMA at the level of L3 based on CSMA at the level of $\mathrm{C} 3$ is not ideal and probably is not sufficient in the future as the most accurate estimation of a patient's total SMM. Indeed, Baracos published an article concluding that using single muscle as a sentinel muscle for whole body SMM is flawed premise. ${ }^{21}$ This problem probably also applies to CSMA on a single CT slice as a representation of whole body skeletal muscle volume. We do believe that at the current time, the CSMA at C3 can provide a good estimation of SMM of HNC patients without the need for additional diagnostics and at minimal effort, with considerable accuracy.

To facilitate implementation of SMM measurement in clinical practice, we believe the long-term focus should shift towards using artificial intelligence such as deep learning and machine learning to develop an automatic, whole muscle volume analysis based on routinely available CT imaging or MRI. Research into these methods are ongoing, and the expectation is that whole- or portion-ofbody measurement of SMM will provide a much more accurate representation of a patients overall body composition and skeletal muscle status than the CSMA on a single CT slide or a single muscle, with no or very little manual work involved. ${ }^{22-24}$ Indeed, the use of the CSMA at the level of L3 as an estimation of whole body skeletal muscle volume is based on studies using whole-body MR for manual segmentation and calculation of whole-body skeletal muscle volume; in these studies, whole body MRI is referenced as the gold standard. ${ }^{6,25}$ Manual segmentation of whole body MRI is time-consuming and therefore clinically not feasible. However, when software is available to perform automatic skeleta muscle volume analysis, a whole-body analysis approach seems preferred. In the short term, future studies may be aimed at developing gender-specific references values for CSMA at the level of C3, to allow for the use of CSMA at the level of C3 as a direct measure of SMM and to overcome the problem of several different cutoffs for low SMM that are currently available..$^{26,27}$

There are limitations to our study that need to be addressed. Most patients in our study presented with advanced stage disease; in our center, the indication for FDG-PET/CT is a suspected advanced stage disease. Inherently to the use of FDG-PET/CT, patients with limited disease are underrepresented in this study. We excluded patients who had received prior treatment for HNC for this validation 
study, because the effect of prior local treatment (e.g. radiotherapy or surgery) on the accuracy of delineation of CSMA at C3 is not known, and may cloud it relationship with CSMA at L3. It is well-known that patients with tobacco-related cancers of the upper aero-digestive tract have a substantial risk of developing a second primary malignancy in the same region. In another study by our group, also imaging of patients who had undergone prior treatment was also used and found that low SMM as identified at the level of C3 was associated with dverse outcomes in patients with and without prior treatment. ${ }^{9}$ Some patients with HNC will undergo MRI instead of CT imaging. In this study we only used CT imaging, according to the protocol described by Swartz et al.7 Two recent studies also showed excellent correspondence between CSMA on CT imaging and MRI and concluded that CT and MRI can be used interchangeably. ${ }^{28,29}$ The effect of different posture and different angles (e.g. in laryngeal cancer, CT scans are often angulated to better visualize the vocal cords) was not evaluated in this study, but may influence CSMA. ${ }^{30}$ Future research should clarify this, but we expect that this problem will be overcome by using whole-body or portion-of-body skeleta muscle volumes using artificial intelligence.

Our current study confirms the previously found strong correlation between CSMA at the level of C3 and CSMA at the level of L3. This method allows for research into the predictive and prognostic effect of low SMM in HNC patients, using routinely performed imaging of the head and neck region without any additiona costs or burden for the patient. It may also be used to identify patients with low SMM at high risk of adverse clinical outcomes, who may benefit from treatmen adaptation or additional supportive treatment; acknowledging that there is some uncertainty in the identification of patients with low SMM.

\section{Conclusion}

A measurement of CSMA at the level of $\mathrm{C} 3$ is a reliable method for evaluation of SMM in HNC patients and allows for investigating the predictive and prognostic value of low SMM in HNC patients using routinely performed CT imaging of the head and neck area. There is reasonable accuracy in the identification of patients with low SMM based on the estimated lumbar SMI and the actual lumbar SMI. Future research should be aimed at optimizing methods to use routinely performed imaging for body composition analysis.

\section{References}

1. Prado CM, Cushen SJ, Orsso CE, Ryan AM. Sarcopenia and cachexia in the era of obesity: Clinical and nutritional impact. Proc Nutr Soc 2016;75:188-98.

2. Martin L, Birdsell L, MacDonald N, Reiman T, Clandinin MT, McCargar LJ, et al. Cancer cachexia in the age of obesity: Skeletal muscle depletion is a powerful prognostic factor, independen of body mass index. J Clin Oncol 2013;31:1539-47.

3. Levolger S, van Vugt JLA, de Bruin RWF, IJzermans JNM. Systematic review of sarcopenia in patients operated on for gastrointestinal and hepatopancreatobiliary malignancies. Br J Surg 2015;102:1448-58.

4. Rier HN, Jager A, Sleijfer S, Maier AB, Levin M. The Prevalence and Prognostic Value of Low Muscle Mass in Cancer Patients: A Review of the Literature. Oncologist 2016;21:1396-409.

5. Prado CM, Lieffers JR, McCargar LJ, Reiman T, Sawyer MB, Martin L, et al. Prevalence and clinical implications of sarcopenic obesity in patients with solid tumours of the respiratory and gastrointestinal tracts: a population-based study. Lancet Oncol 2008;9:629-35.

6. Shen W, Punyanitya M, Wang Z, Gallagher D, St.-Onge M-P, Albu J, et al. Total body skeletal muscle and adipose tissue volumes: estimation from a single abdominal cross-sectional image. J Appl Physiol 2004;97:2333-8.

7. Swartz JE, Pothen AJ, Wegner I, Smid EJ, Swart KMA, de Bree R, et al. Feasibility of using head and neck CT imaging to assess skeletal muscle mass in head and neck cancer patients. Ora Oncol 2016;62:28-33.

8. Wendrich AW, Swartz JE, Bril SI, Wegner I, De Graeff A, Smid EJ, et al. Low skeletal muscle mass is a predictive factor for chemotherapy dose-limiting toxicity in patients with locally advanced head and neck cancer 2017.

9. Bril SI, Pezier TF, Tijink BM, Janssen LM, Braunius WW, Bree R. Preoperative low skeletal muscle mass as a risk factor for pharyngocutaneous fistula and decreased overall survival in patients undergoing total laryngectomy. Head Neck 2019;41:1745-55.

10. Chargi N, Bril SI, Swartz JE, Wegner I, Willems SW, de Bree R. Skeletal muscle mass is an imaging biomarker for decreased survival in patients with oropharyngeal squamous cell carcinoma. Oral Oncol 2020;101.

11. Achim V, Bash J, Mowery A, Guimaraes AR, Li R, Schindler J, et al. Prognostic Indication of . 2017;143:1159.

12. Ansari E, Chargi N, van Gemert JTM, van Es RJJ, Dieleman FJ, Rosenberg AJWP, et al. Low skeleta muscle mass is a strong predictive factor for surgical complications and a prognostic factor in ral cancer patients undergoing mandibular reconstruction with a free fibula flap. Oral Onco

van Rijn-Dekker MI, van den Bosch L, van den Hoek JGM, Bijl HP, van Aken ESM, van der Hoorn A, et al. Impact of sarcopenia on survival and late toxicity in head and neck cancer patients treated with radiotherapy. Radiother Oncol 2020.

14. Ufuk F, Herek D, Yüksel D. Diagnosis of sarcopenia in head and neck computed tomography: Cervical muscle mass as a strong indicator of Sarcopenia. Clin Exp Otorhinolaryngol 2019.

15. B. Heymsfield S, Wang Z, Baumgartner RN, Ross R. Human Body Composition: Advances in Models and Methods. Annu Rev Nutr 1997;17:527-58.

16. Prado CMM, Heymsfield SB. Lean Tissue Imaging. J Parenter Enter Nutr 2014;38:940-53.

17. Bol GH, Kotte ANTJ, van der Heide UA, Lagendijk JJW. Simultaneous multi-modality ROI delineation in clinical practice. Comput Methods Programs Biomed 2009;96:133-40.

Reliability Research. J Chiropr Med 2016;15:155-63.

9. Landis JR, Koch GG. The Measurement of Observer Agreement for Categorical Data. Biometrics 1977.

20. Bril SI, Wendrich AW, Swartz JE, Wegner I, Pameijer F, Smid EJ, et al. Interobserver agreement of skeletal muscle mass measurement on head and neck CT imaging at the level of the third cervical vertebra. Eur Arch Oto-Rhino-Laryngology 2019;276:1175-82 
21. Baracos VE. Psoas as a sentinel muscle for sarcopenia: a flawed premise. J Cachexia Sarcopenia Muscle 2017. Weston AD, Korfiatis P, Kline TL, Philbrick KA, Kostandy P, Sakinis T, et al. Automated Abdomina Segmentation of CT Scans for Body Composition Analysis Using Deep Learning. Radiology

23. Koitka S, Kroll L, Malamutmann E, Oezcelik A, Nensa F. Fully-automated body composition analysis in routine ct imaging using $3 \mathrm{~d}$ semantic segmentation convolutional neural networks. ArXiv 2020.

24. Blanc-Durand P, Campedel L, Mule S, Jegou S, Luciani A, Pigneur F, et al. Prognostic value of anthropometric measures extracted from whole-body $\mathrm{CT}$ using deep learning in patients with

25. Schweitzer L, Geisler C, Pourhassan M, Braun W, Glüer CC, Bosy-Westphal A, et al. What is the best reference site for a single MRI slice to assess whole body skeletal muscle and adipose tissue volumes in healthy adults? Am J Clin Nutr 2015.

26. van Vugt JLA, van Putten $Y$, van der Kall IM, Buettner S, D'Ancona FCH, Dekker HM, et al. Estimated skeletal muscle mass and density values measured on computed tomography examinations in over 1000 living kidney donors. Eur J Clin Nutr 2019.

27. Van Der Werf A, Langius JAE, De Van Der Schueren MAE, Nurmohamed SA, Van Der Pant KAMI, Blauwhoff-Buskermolen $\mathrm{S}$, et al. Percentiles for skeletal muscle index, area and radiation attenuation based on computed tomography imaging in a healthy Caucasian population. Eur $J$ Clin Nutr 2018;72:288-96.

28. Zwart AT, Becker JN, Lamers MJ, Dierckx RAJO, de Bock GH, Halmos GB, et al. Skeletal muscle mass and sarcopenia can be determined with 1.5-T and 3-T neck MRI scans, in the event that no neck CT scan is performed. Eur Radiol 2020.

29. Chargi N, Ansari E, Huiskamp LFJ, Bol G, de Bree R. Agreement between skeletal muscle mass measurements using computed tomography imaging and magnetic resonance imaging in head and neck cancer patients. Oral Oncol 2019;99:104341.

30. Baum U, Greess H, Lell M, Nomayr A, Lenz M. Imaging of head and neck tumors - Methods: CT, spiral-CT, multislice-spiral-CT. Eur J Radiol 2000. 


\section{Chapter 3}

Interobserver agreement of skeletal muscle mass measurement on head and neck CT imaging at the level of the third cervical vertebra

Sandra I. Bril, Anne W. Wendrich, Justin E. Swartz, Inge Wegner, Frank Pameijer, Ernst J. Smid, Gijs H. Bol, Ajit J. Pothen, Remco de Bree 


\section{Abstract}

Objectives Skeletal muscle mass (SMM) is most often assessed in cancer patients on abdominal computed tomography (CT) imaging at the level of the third lumbar vertebra (L3). Abdominal CT imaging is not routinely performed in head and neck cancer (HNC) patients. Recently, a novel method to assess SMM on a single transversal CT slice at the level of the third cervical vertebra (C3) was published. The objective of this study was to assess the robustness of this nove C3 measurement method in terms of interobserver agreement.

Methods Patients diagnosed with locally advanced head and neck squamous cell carcinoma (LA-HNSCC) at our center between 2007 and 2011 were evaluated. Fifty-four patients with were randomly selected for analysis. Six observers independently measured the cross-sectional muscle area (CSMA) at the level of C3 using a predefined, written protocol as instruction. Interobserver agreement was assessed using intraclass correlation coefficients (ICCs), a Bland-Altman plot and Fleiss' kappa (к).

Results The agreement in vertebra selection between all observers was excellent (Fleiss' $\mathrm{k}$ : 0.96). There was a substantial agreement between all observers in single slice selection (Fleiss' $\mathrm{k:}$ 0.61). For all CSMA measurements, ICCs were excellent 0.763-0.969; all $\mathrm{p}<0.001)$. The Bland-Altman plot showed good agreement between measurements, with narrow limits of agreement.

Conclusion Interobserver agreement for SMM measurement at the level of C3 was excellent. Assessment of SMM at the level of C3 is easy and robust and can performed on routinely available imaging in HNC patients.

\section{Introduction}

Body composition increasingly is a subject of interest in medical research. An abnormal body composition, such as a decreased lean body mass and/or increased adipose tissue mass, may have a profound influence on treatment outcome and (disease free) survival of patients with a variety of illnesses ${ }^{1-3}$. Skeletal muscle mass (SMM) is the largest component of lean body mass. In cancer patients, a low a higher incidence of chemotherapy related toxicity, postoperative complications, longer hospital stay, increased healthcare related expenditures and lower disease free and overall survival..$^{5-10}$ This relationship has been shown in breast, colorectal, hepato-pancreatico-biliary, renal and lung cancer, amongst others. ${ }^{11-15}$

In cancer patients, SMM is most often assessed on abdominal computed tomography (CT) imaging at the level of the third lumbar vertebra (L3)..$^{16}$ This method is based on research using whole-body magnetic resonance imaging (MRI), in which has been shown that cross-sectional skeletal muscle area (CSMA) on a single transversal slice at the level of L3 is strongly correlated with total skeletal muscle volume as measured using whole-body MRI. ${ }^{17,18}$ The CSMA at the level of $L 3$ is commonly normalized for stature, which results in the lumbar skeletal muscle index (lumbar SMI). ${ }^{18}$ This value is used as an indication of total SMM.

Abdominal CT imaging is often routinely performed in most types of cancer during diagnostic work-up and follow-up. In these patients, SMM measurement can be performed on abdominal CT imaging without the need for additional imaging or other diagnostics. However, abdominal CT imaging is not routinely performed in head and neck cancer (HNC) patients. ${ }^{19}$ Recently, a novel method to assess SMM on a single transversal CT slice at the level of the third cervical vertebra (C3) was published. ${ }^{20}$ Using this method, skeletal muscle mass is assessed measuring the CSMA of the paravertebral muscles and the sternocleidomastoid muscles at the level of the C3 vertebra. This method allows for evaluation of SMM in HNC patients on routinely performed imaging, in a similar manner as is used in patients with other types of cancer. This measurement method for SMM was recently used in three studies in head and neck cancer patients..$^{21-23}$

In order to be clinically useful, the C3 measurement method of SMM has to provide similar results when used by different observers. The aim of this study was to evaluate the interobserver agreement of SMM measurement at the level of C3. The robustness of the C3 measurement method was investigated in terms of 
the vertebra selection, the exact single slice selection, and the correspondence in CSMA measurements between all observers.

\section{Methods}

\section{Ethical approval}

The design of this study was approved by the Medical Ethical Research Committee of our center (approval ID 14-544/C). All data was retrieved retrospectively. Measurements of SMM were performed on coded CT scans.

\section{Patients and study design}

Patients diagnosed with locally advanced head and neck squamous cell carcinom (LA-HNSCC) at the our center between 2007 and 2011 were evaluated for this study. Fifty-four patients with pre-treatment head and neck CT imaging were randomly selected for inclusion. Other parameters, including length and weight at the time of imaging, sex, age, tumor localization and clinical TNM stage (7th edition) were retrospectively retrieved. ${ }^{24}$

\section{Imaging protocol and analysis}

All patients underwent contrast-enhanced $\mathrm{CT}$ scanning prior to radiation treatment on a Philips Brilliance iCT scanner (Philips Healthcare, Best, the Netherlands) using a standardized protocol for HNC patients. The imaging was performed in treatment position in a radiotherapy immobilization mask. Scanning parameters included slice thickness $1 \mathrm{~mm}$ with a $2 \mathrm{~mm}$ interslice gap.

\section{Measurement of SMM}

Delineation of CSMA was manually performed using the Volumetool v.1.6.5 Research Software Package, designed in our center as an image evaluation registration and delineation system for radiotherapy planning ${ }^{25}$, Hounsfield Unit (HU) thresholds for skeletal muscle tissue were -29 and $+150 \mathrm{HU}$; the area within these HU thresholds was defined as the CSMA. Delineation of CSMA was performed independently by six observers; 1 experienced head and neck radiologist, 1 experienced head and neck radiation oncologist, and 4 medically trained researchers from the departments of Head and Neck Surgical Oncology, and Otorhinolaryngology and Head \& Neck Surgery. A predefined, written protocol for single slice selection and CSMA was provided, as described in the recently published study by Swartz et al. ${ }^{20}$ In brief, the first slice when scrolling from caudal to cranial direction to show both transverse processes and the entire vertebral arc of the third cervical vertebra had to be selected. Contours of the paravertebral muscles (PVM) and both sternocleidomastoid (SCM) muscles were manually traced. This study was performed without a training dataset, as to simulate the use of this measurement method as if it were adopted from an external research paper.

All observers independently selected the slice they deemed to be correct from the CT scan, and delineated CSMA. Observers were blinded to their own anc other's results. After delineation of CSMA at C3 was finished by all observers, CSMA was automatically retrieved from Volumetool. Vertebra and single slice selection was investigated by reopening all scans after CSMA retrieval and analysis was finished; the selected vertebra and single slice location were noted. Total CSMA at $\mathrm{C} 3$ was calculated as the sum of the CSMA of the PVM and both SCM muscles. Figure 1 shows an example of CSMA delineation at the level of C3.

\section{Figure 1: example of delineation of skeletal muscle at the level of C3}

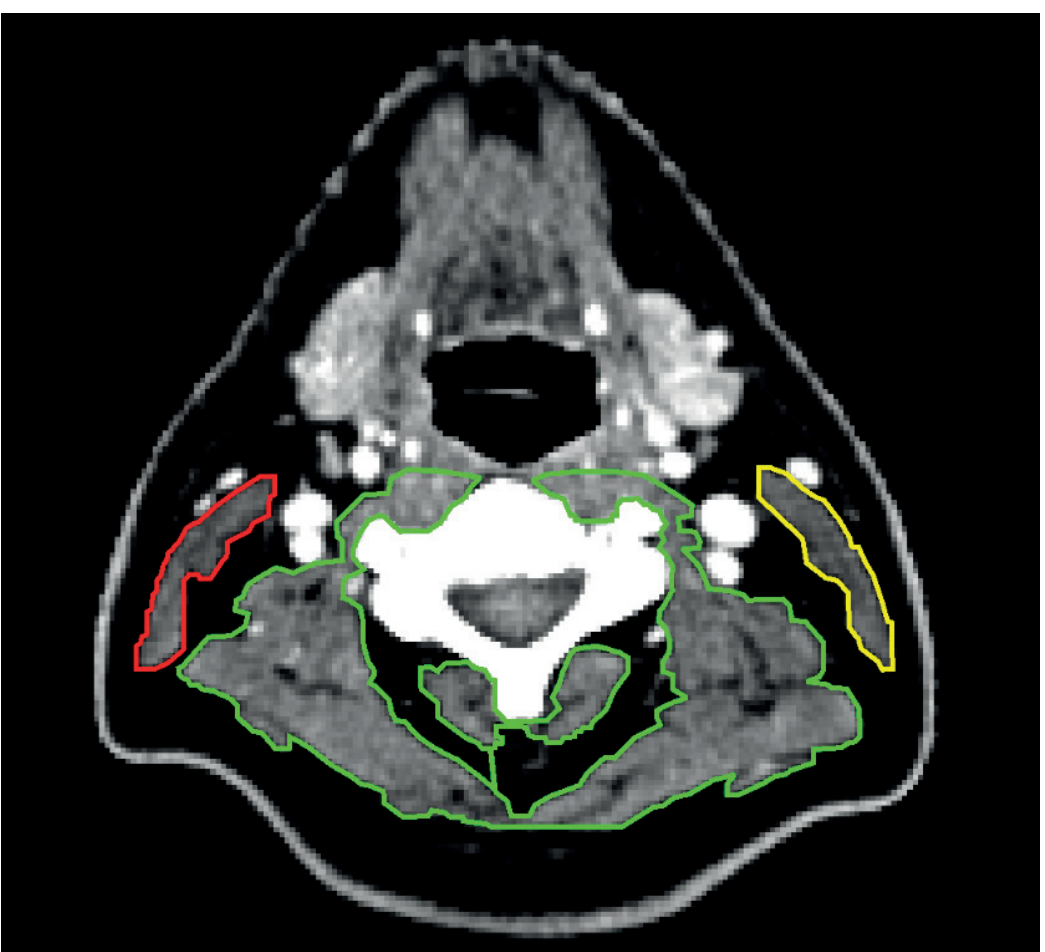

The paravertebral muscles are delineation in green. The left sternocleidomastoid muscle is delineated in yellow, and the right sternocleidomastoid muscle in red. 
All statistical analyses were performed using the IBM SPSS Statistics version 21.0 software package (Chicago, Illinois, USA). A test for normality (Shapiro-Wilk test) was used to assess whether continuous variables were normally distributed. Continuous data are represented as mean \pm standard deviation (SD). Categorical data are represented as a number and percentage of total. Differences in skeletal muscle area measurements between observers were calculated using a repeated measures ANOVA. Agreement between different observers was assessed by calculating Fleiss' kappa ( $\mathrm{K}$ ) and intraclass correlation coefficients (ICCs) using a two-way mixed single measures model with absolute agreement and. The $k$ values were graded as slight $(0.01-0.20)$, fair (0.21-0.40), moderate (0.41-0.60), substantial $(0.61-0.80)$ or almost perfect $(0.81-0.99)$ agreement. ${ }^{26}$ The ICCs were rated as poor $(0.00-0.49)$, fair to good $(0.50-0.74)$ and excellent $(0.75-1.00)$. Bland-Altman plots were constructed to visualize agreement. ${ }^{28}$ Results were considered statistically significant if the $p$-value was less than 0.05

\section{Results}

\section{Patient characteristics}

Baseline characteristics of the 54 included patients are shown in Table 1. Patients were predominantly male and mostly presented with lymph node positive, stage IV disease.

\section{Vertebra selection}

Table 2 shows the results of the vertebra and single slice selection analysis. For the vertebra selection, the overall correspondence between all observers was near perfect (Fleiss' $\mathrm{k:} 0.96, \mathrm{p}<0.001$ ). The correct vertebra (C3) was identified in almost all patients by all observers; in 5 patients one observers chose a different vertebra, and in 1 patient two observers chose a different vertebra. Either $\mathrm{C} 2$ or $\mathrm{C} 4$ was selected in these patients.
Table 1: Baseline patient characteristics

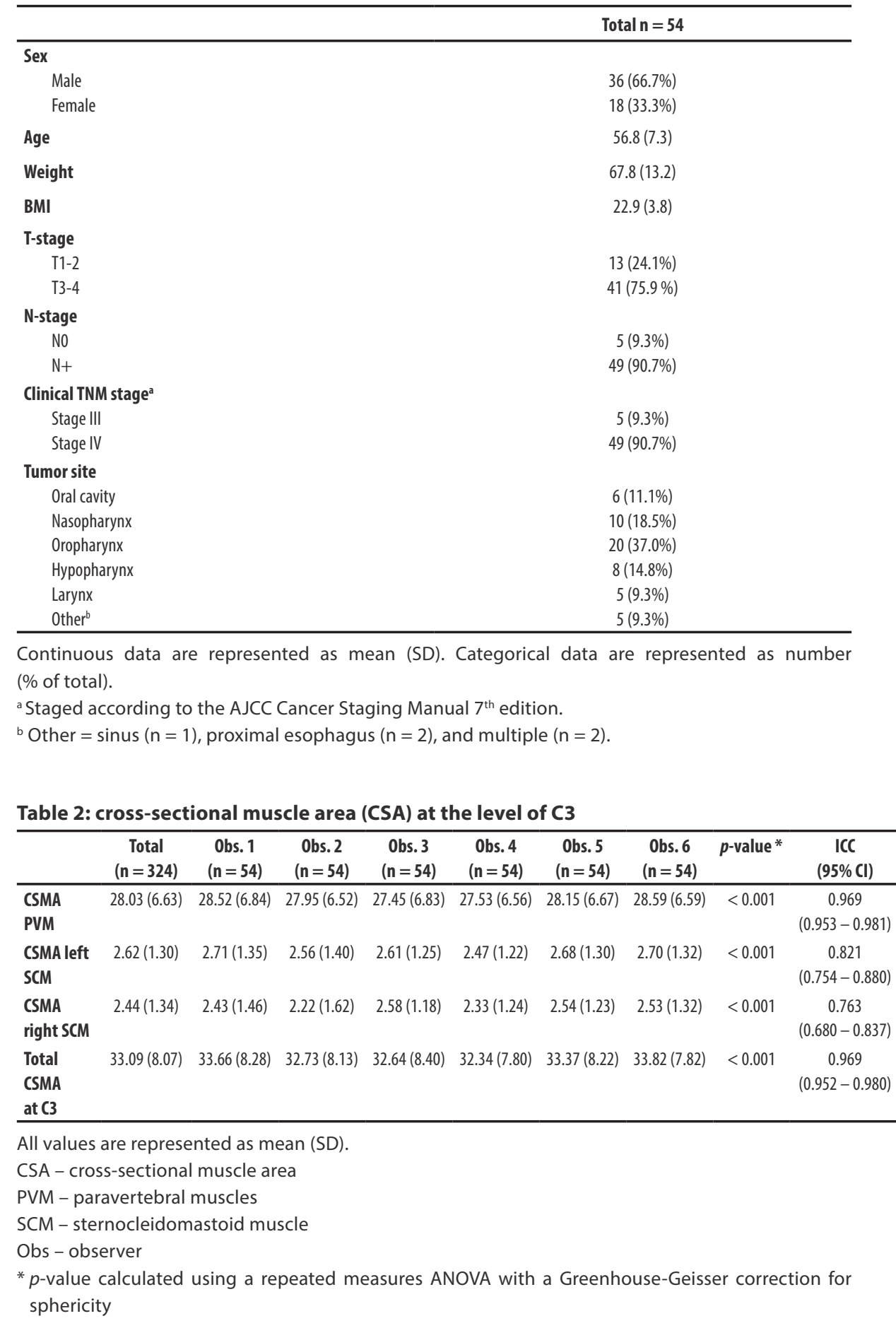




\section{Single slice selection}

There was more variation in exact single slice selection. The overal correspondence between all observers was substantial (Fleiss' $\mathrm{k}: 0.61, \mathrm{p}<0.001$ )

In $22.2 \%$ of patients, all observers chose the same identical slice to delineate; while in the other $77.8 \%$, at least one observer chose a different slice. In $79.3 \%$ of the cases where a different slice from the majority was chosen, the different slice was located directly above or below the slice the majority of observers chose.

\section{Table 3: vertebra and single CT slice selection}

\begin{tabular}{lcc}
\hline & Vertebra $(\mathrm{n}=54)$ & Slice $(\mathrm{n}=54)$ \\
\hline All observers same & $49(88.9 \%)$ & $12(22.2 \%)$ \\
One observer different & $5(9.1 \%)$ & $16(29.6 \%)$ \\
Two observers different & $1(1.9 \%)$ & $12(22.2 \%)$ \\
Three observers different & - & $14(25.9 \%)$ \\
\hline
\end{tabular}

Values shown as number (\% of total).

\section{Cross-sectional skeletal muscle area measurements}

Mean CSMA of the PVM, left SCM and right SCM, and of total CSMA at C3 are shown in Table 2. Most CSMA measurements were normally distributed (ShapiroWilk: $p>0.05$ ). There was a significant difference between the observers in SSMA measurements (repeated measures ANOVA: $p<0.001$ ). Actual differences between CSMA measurements of all observers were small; the largest mean difference between observers was $1.48 \mathrm{~cm}^{2}$ (observer 6 - observer 4). For all CSMA measurements, ICC's were excellent $(0.763-0.969$; all $p<0.001)$, showing good conformity between measurements of different observers, as is visualized in Figure 2.

Figure 3 shows a combined Bland-Altman plot of total CSMA at C3 measurement of the individual observers and their difference to the overall mean CSMA at C3 measurements. The mean of the standard deviation of the difference between observer CSMA measurements and mean CSMA measurement was used to calculate the limits of agreement (95\% confidence interval). There appears to be an element of systemic bias in CSMA measurement at C3, with some observer having more than $5 \%$ measurements outside of the $95 \%$ limits of agreement, and some observers systemically deviate one way (higher or lower) from the mean CSMA measurement. Actual differences are small.
Figure 2: intra-class correlation plot for measurement of total CSMA measurement at C3
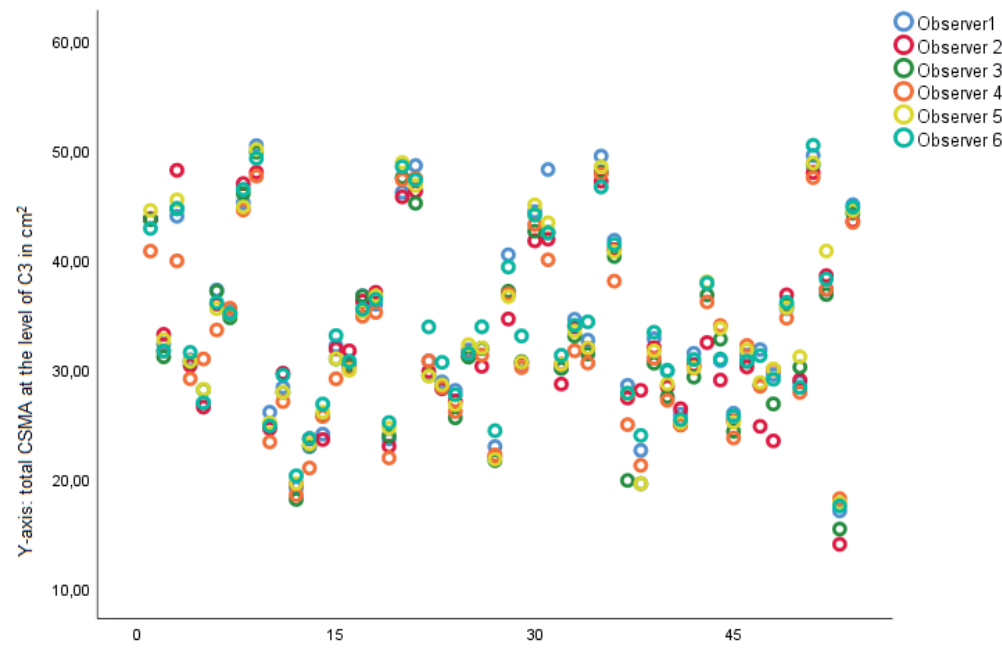

-axis: patient number

The correspondence between measurements is visualized in the intra-class correlation plot.

Figure 3: Bland Altman plot for agreement of total CSMA measurement at C3 between all observers

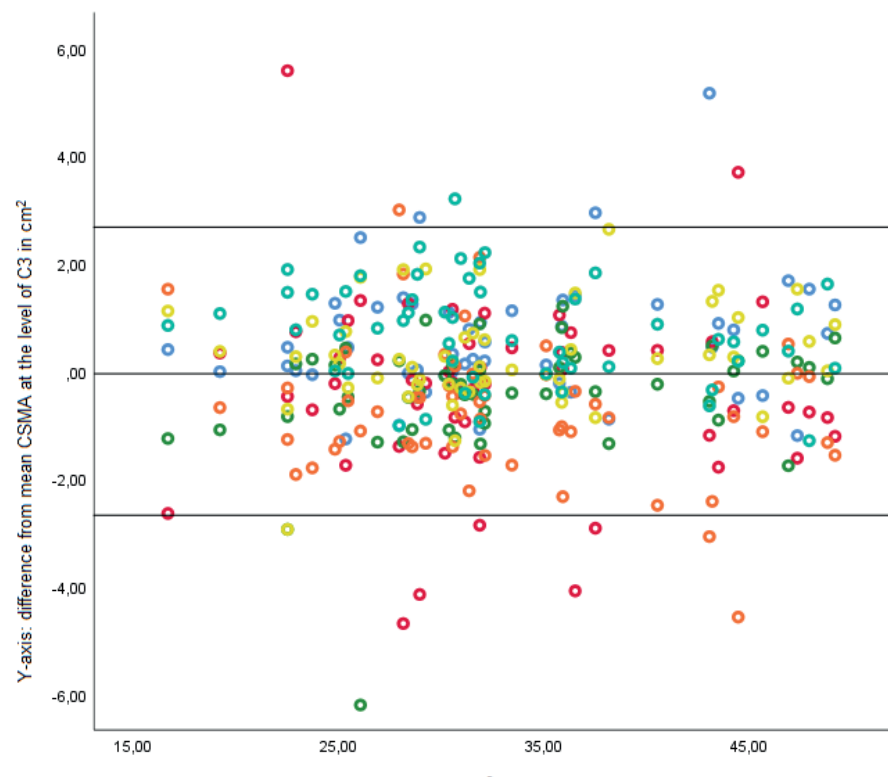

(150)

Bland-Altman plots showing agreement between measurements of observers. The solid lines depict the mean and $95 \%$ limits of agreement in the Bland Altman plot. 


\section{Discussion}

This study aimed to examine the robustness of the $\mathrm{C} 3$ measurement method for SMM in terms of interobserver agreement and specific vertebra and single slice selection by multiple observers. This study was performed without a training dataset and limited formal training, in order to simulate the use of the C3 measurement method for SMM as if it were adopted from an external research paper.

The interobserver agreement for all different CSMA measurements was excellent. The measurement of CSMA of PVM appears to be most uniform. There is some difference between observers in measurement of the CSMA of the SCM muscles; however, actual differences remain small. Previous research has shown that total CSMA at C3 was significantly better correlated with CSMA at L3 than the CSMA of the PVM only with CSMA at L3. ${ }^{20}$

The level of C3 is easily identifiable for both researchers and clinicians, with only incidental selection of a different level. There was more variation in the actual single slice selection, perhaps due to the lack of a training dataset and little informal training received prior to delineation of scans. This may also be due to the thin slices $(1 \mathrm{~mm})$ and small $(2 \mathrm{~mm})$ interslice gaps of the CT imaging used in this study. In most cases where an observer had selected a different slice than the majority of the observers, the different slice was indeed directly above or below of the slice the majority chose, which corresponds with a $3 \mathrm{~mm}$ difference in location. Probably, in these cases the accidental selection of a different slice should not greatly influence CSMA measurements. However, it may still be advisable to include a training dataset when starting to use the C3 measurement method to allow for a learning period in delineation of skeletal muscle and single slice selection.

In recent years, SMM has widely been researched in cancer patients, using a measurement of skeletal muscle area at the level of L3 on CT imaging as an indicator of total SMM. ${ }^{11}$ Imaging at the level of L3 is not routinely performed in HNC patients, so SMM measurement at the level of L3 is not always clinically applicable in HNC. ${ }^{19}$ The SMM measurement method at the level of C3 provides a reliable and robust alternative to SMM measurement at the level of L3, allowing for broad research into the predictive and prognostic effect of sarcopenia in HNC.
Patients with HNC often present with signs of malnutrition, and as such are at risk of developing sarcopenia. ${ }^{29}$ Adverse outcomes associated with sarcopenia, such as chemotherapy related toxicity and wound healing problems, are highly prevalent in HNC. ${ }^{30}$ It can be anticipated that both are at least partly related to sarcopenia. The first study using the C3 measurement method to assess SMM in HNC patients undergoing chemoradiotherapy found that low SMM was an independent predictor of the occurrence of chemotherapy dose-limiting toxicity. ${ }^{21} \mathrm{~A}$ recent study in HNC patients undergoing laryngectomy for laryngeal cancer showed that low SMM, as measured at the level of L3, was an independent predictor of the occurrence of a pharyngocutaneous fistula and of the occurrence of any wound complication..$^{31}$ In this study 122 patients who had undergone a total laryngectomy were retrospectively evaluated for inclusion; 70 (57\%) had abdominal imaging available for analysis. It is likely that all or almost all HNC patients will undergo CT or MRI imaging of the head and neck area during the diagnostic process and follow-up. ${ }^{19}$ The $\mathrm{C} 3$ measurement method allows the investigation of body composition in nearly all HNC patients without the need for extra diagnostics. A recent study in patients undergoing laryngectomy where SMM was measured at the level of C3 showed that low SMM was a predictor of the occurrence of a pharyngocutaneous fistula. ${ }^{23}$ Preoperative low SMM also was a strong negative prognostic factor for overall survival after laryngectomy. In this retrospective study, 235 out of 245 patients could be included for analysis due to measurement of SMM at the level of $\mathrm{C} 3 .^{23}$

Future research is still needed to clarify whether the adverse effects of low SMM are prognostic only, or if these adverse effects can be overturned. In the future, HNC patients at high risk of adverse outcomes related to low SMM or sarcopenia might benefit from additional supportive treatment or individualized primary treatment. Several possible interventions may be considered, such as altered chemotherapy dosing ${ }^{32}$, prehabilitation before surgery ${ }^{33}$, enhanced recovery after surgery ${ }^{34}$, intensive physiotherapy ${ }^{35}$ and additional nutritional support ${ }^{36}$. A measurement of SMM at the level of C3 might be used as a screening tool for low SMM in HNC cancer patients at diagnosis.

\section{Limitations}

There are some limitations to this study that need to be addressed. No training dataset or formal training was given to the observers in this study. Although this study set-up was chosen because it may best mimic the clinical adaptation and use of the $\mathrm{C} 3$ measurement method by other institutions, it may also explain some of the differences in the measurements and in the vertebra and single 
slice selections. For further use in research and clinical work, a training dataset for researchers learning skeletal muscle area measurement may limit these differences. Secondly, only CT imaging was used in this study to assess SMM, as is usually done in other studies in cancer patients that assess SMM at the level of 3. Some HNC patients will only undergo MRI imaging of the head and neck area during the diagnostic process. It should still be evaluated whether CT and MRI can be used interchangeably for SMM assessment or that some form of modification of the method is necessary. Lastly, most variation in CSA measurements was seen in the SCM muscles. This can at least partially be explained by lymph node metastasis close to or invading in the SCM muscles. Most patients included in this study had advanced, lymph node positive disease, which may make accurate delineation of the muscle more difficult. In daily clinical practice, approximately two-thirds of HNC patients present with advanced stage disease (large tumour and/or lymph node positive). ${ }^{37}$ Thus, this study population provides an accurate reflection of the clinical use of the $\mathrm{C} 3$ measurement method. Because previous research showed that addition of the CSA of the SCM muscles was beneficial for a prediction model, and because actual differences were small and ICC's could stil be classified as excellent, it is justified to include the CSA of the SCM muscles in the total CSA at C3 in patients with lymph node positive disease.

\section{Conclusion}

Interobserver agreement is excellent for SMM measurement on head and neck $\mathrm{CT}$ imaging at the level of $\mathrm{C} 3$, as tested in a setting that mimics a clinical setting. The C3 measurement method for SMM is robust and easy to use, using routinely performed CT imaging of the head and neck area. It allows for retrospective and prospective research into the predictive and prognostic value of low SMM in the vast majority of HNC patients, as well as use in possible future trials.

\section{References}

1. Cruz-Jentoft AJ, Baeyens JP, Bauer JM, Boirie Y, Cederholm T, Landi F, et al (2010) Sarcopenia European consensus on definition and diagnosis: Report of the European Working Group on Sarcopenia in Older People. Age Ageing 39(4):412-23.

2. Wannamethee SG, Atkins $\mathrm{JL}$ (2015) Muscle loss and obesity: the health implications of sarcopenia and sarcopenic obesity. Proc Nutr Soc 74(4):405-12.

3. Janssen I, Heymsfield SB, Ross R (2002) Low relative skeletal muscle mass (sarcopenia) in older persons is associated with functional impairment and physical disability. J Am Geriatr So 50(5):889-96.

4. Prado CM, Heymsfield SB (2014) Lean tissue imaging: a new era for nutritional assessment and intervention. JPEN J Parenter Enteral Nutr 38(8):940-53.

5. Rier HN, Jager A, Sleijfer S, Maier AB, Levin MD (2016) The Prevalence and Prognostic Value of Low Muscle Mass in Cancer Patients: A Review of the Literature. Oncologist 21(11):1396-1409. Shachar SS, Williams GR, Muss HB, Nishijima TF (2016) Prognostic value of sarcopenia in adults with solid tumours: A meta-analysis and systematic review. Eur J Cancer 57:58-67.

7. Levolger S, van Vugt JL, de Bruin RW, IJzermans JN (2015) Systematic review of sarcopenia in patients operated on for gastrointestinal and hepatopancreatobiliary malignancies. Br J Surg 102(12):1448-58.

8. Jones K, Gordon-Weeks A, Coleman C, Silva M (2017) Radiologically Determined Sarcopenia Predicts Morbidity and Mortality Following Abdominal Surgery: A Systematic Review and Meta-Analysis. World J Surg 41(9):2266-2279.

9. Lieffers JR, Bathe OF, Fassbender K, Winget M, Baracos VE (2012) Sarcopenia is associated with postoperative infection and delayed recovery from colorectal cancer resection surgery. $\mathrm{Br}$ Cancer 107(6):931-6.

10. Van Vugt JLA, Buettner S, Levolger S, Coebergh van der Braak RRJ, Suker M, Gaspersz MP, et a (2017) Low skeletal muscle mass is associated with increased hospital expenditure in patients undergoing cancer surgery of the alimentary tract. PLoS One 12(10):e0186547.

11. Prado CM, Lieffers JR, McCargar LJ, Reiman T, Sawyer MB, Martin L, et al (2008) Prevalence and clinical implications of sarcopenic obesity in patients with solid tumours of the respiratory and gastrointestinal tracts: a population-based study. Lancet Oncol 9(7):629-35.

12. Mourtzakis M, Prado CM, Lieffers JR, Reiman T, McCargar LJ, Baracos VE (2008) A practical and precise approach to quantification of body composition in cancer patients using computed tomography images acquired during routine care. App Physiol Nutr Metab 33(5).997-1006.

3. Sjoblom B, Gronberg BH, Benth JS, Baracos VE, Flotten O, Hjermstad MJ, et al (2015) Low muscle mass is associated with chemotherapy-induced haematological toxicity in advanced nonsmall cell lung cancer. Lung Cancer 90(1):85-91.

14. Cushen SJ, Power DG, Teo MY, MacEneaney P, Maher MM, McDermott R, et al (2017) Body Composition by Computed Tomography as a Predictor of Toxicity in Patients With Rent Cell Carcinoma Treated With Sunitinib. Am J Clin Oncol 40(1):47-52.

Levolger S, van Vledder MG, Muslem R, Koek M, Niessen WJ, de Man RA, et al (2015) Sarcopenia impairs survival in patients with potentially curable hepatocellular carcinoma. Journal of surgical oncology 112(2):208-13.

16. Carneiro IP, Mazurak VC, Prado CM (2016) Clinical Implications of Sarcopenic Obesity in Cancer. Curr Oncol Rep 18(10):62.

17. Shen W, Punyanitya M, Wang Z, Gallagher D, St-Onge MP, Albu J, et al (2004) Total body skeletal muscle and adipose tissue volumes: estimation from a single abdominal cross-sectional image. J Appl Physiol (1985) 97(6):2333-8.

18. Schweitzer L, Geisler C, Pourhassan M, Braun W, Gluer CC, Bosy-Westphal A, et al (2015) What is the best reference site for a single MRI slice to assess whole-body skeletal muscle and adipose tissue volumes in healthy adults? Am J Clin Nutr 102(1):58-65.

19. Gregoire V, Lefebvre JL, Licitra L, Felip E, Group E-E-EGW (2010) Squamous cell carcinoma of the head and neck: EHNS-ESMO-ESTRO Clinical Practice Guidelines for diagnosis, treatment and follow-up. Ann Oncol 21 Suppl 5:v184-6. 
20. Swartz JE, Pothen AJ, Wegner I, Smid EJ, Swart KM, de Bree R, et al (2016) Feasibility of using head and neck CT imaging to assess skeletal muscle mass in head and neck cancer patients. Oral oncology 62:28-33. Wendrich AW, Swartz JE, Bril SI, Wegner I, de Graeff A, Smid EJ, et al (2017) Low skeletal muscle mass is a predictive factor for chemotherapy dose-limiting toxicity in patients with locally

22. Bozkurt G, Elhassan HA, Mahmutoglu AS, Çelebi I, Mcleod RWJ, Soyta's P, et al (2018) Neck Muscle Mass Index as a Predictor of Post-Laryngectomy Wound Complications. Ann Otol

23. Bril SI, Pezier TF, Tijink BM, Janssen LM, Braunius WW, de Bree R (2018) Preoperative low skeleta muscle mass as a risk factor for pharyngocutaneous fistula and decreased overall survival in patients undergoing total laryngectomy. Head Neck. 2019 Jun;41(6):1745-1755.

24. Edge SB, Compton CC (2010) The American Joint Committee on Cancer: the 7th edition of the AJCC cancer staging manual and the future of TNM. Ann Surg Oncol 17(6):1471-4.

25. Bol GH, Kotte A, Lagendijk JJW (2003) Volumetool. An image evaluation, registration, and delineation system for radiotherapy. Phys Med 2003:19.

26. Landis JR, Koch GG (1977) The measurement of observer agreement for categorical data. Biometrics 33(1):159-74.

27. Shrout PE, Fleiss JL (1979) Intraclass correlations: uses in assessing rater reliability. Psychol Bull 86(2):420-8.

28. Bland JM, Altman DG (1986) Statistical methods for assessing agreement between two methods of clinical measurement. Lancet 1(8476):307-10

29. Hebuterne X, Lemarie E, Michallet M, de Montreuil CB, Schneider SM, Goldwasser F (2014) Prevalence of malnutrition and current use of nutrition support in patients with cancer. JPEN Parenter Enteral Nutr 38(2):196-204.

30. Beijer YJ, Koopman M, Terhaard CH, Braunius WW, van Es RJ, de Graeff A (2013) Outcome and toxicity of radiotherapy combi ned with chemotherapy or cetuximab for head and neck cancer: Our experience in one hundred and twenty-five patients. Clin Otolaryngol 38(1):69-74.

31. Achim V, Bash J, Mowery A, Guimaraes AR, Li R, Schindler J, et al (2017) Prognostic Indication of Sarcopenia for Wound Complication After Total Laryngectomy. JAMA Otolaryngol Head Neck Surg 143(12):1159-1165.

32. Sjoblom B, Benth JS, Gronberg BH, Baracos VE, Sawyer MB, Flotten O, et al (2017) Drug Dose Per Kilogram Lean Body Mass Predicts Hematologic Toxicity From Carboplatin-Doublet Chemotherapy in Advanced Non-Small-Cell Lung Cancer. Clin Lung Cancer 18(2):e129-e136.

33. Huang J, Lai Y, Zhou X, Li S, Su J, Yang M, et al (2017) Short-term high-intensity rehabilitation in radically treated lung cancer: a three-armed randomized controlled trial. J Thorac Dis 9(7):1919-1929.

34. Dort JC, Farwell DG, Findlay M, Huber GF, Kerr P, Shea-Budgell MA, et al (2017) Optima Perioperative Care in Major Head and Neck Cancer Surgery With Free Flap Reconstruction: A Consensus Review and Recommendations From the Enhanced Recovery After Surgery Society. JAMA Otolaryngol Head Neck Surg 143(3):292-303.

35. Lonbro S, Dalgas U, Primdahl H, Overgaard J, Overgaard K (2013) Feasibility and efficacy of progressive resistance training and dietary supplements in radiotherapy treated head and neck cancer patients--the DAHANCA 25A study. Acta Oncol 52(2):310-8.

36. Weed HG, Ferguson ML, Gaff RL, Hustead DS, Nelson JL, Voss AC (2011) Lean body mass gain in patients with head and neck squamous cell cancer treated perioperatively with a proteinand energy-dense nutritional supplement containing eicosapentaenoic acid. Head Neck 33(7):1027-33.

37. Argiris A, Karamouzis MV, Raben D, Ferris RL (2008) Head and neck cancer. Lancet 17;371(9625):1695-709 


\section{Chapter 4}

Total skeletal muscle volume estimation from a single magnetic resonance imaging slice of the head and neck area in healthy adults

Sandra I. Bril, Corinna Geisler, Lisa Schweitzer, Anja Bosy Westphal, Pim A. de Jong, Remco de Bree, Manfred J Müller 


\section{Abstract}

Background Head and neck imaging is a common procedure in oncology, neurovascular disease, trauma and infections. This provides the opportunity to estimate total skeletal muscle volume, which is relevant in frailty assessment and as a predictive and prognostic marker for treatment outcome. We aimed to validate skeletal muscle volume estimation on a single magnetic resonance imaging (MRI) slice at the level of the third cervical vertebra (C3) with whole-body MRI measured skeletal muscle volume as the reference standard.

Methods Whole-body MRI was performed in 140 healthy adult volunteers (67 males; mean \pm SD: age $37.0 \pm 11.8$ years; body mass index in $\left.\mathrm{kg} / \mathrm{m}^{2}: 25.3 \pm 5.9\right)$. Segmentation of skeletal muscle area at the level of $\mathrm{C} 3, \mathrm{C} 4$ and $\mathrm{C} 5$ was performed. Skeletal muscle area was correlated with total skeletal muscle volume, and using linear regression modeling a formula was established that can estimate total skeletal muscle volume from the skeletal muscle area at the level of C3 on a single MRI slice.

Results Single slice skeletal muscle area at the level of C3, C4 and C5 all had a good correlation with total skeletal muscle volume $(r>0.87$, all $\mathrm{p}<0.01)$. Skeletal muscle area at C3 correlated best $(r=0.892)$. The univariable formula total skeletal muscle volume $=$ skeletal muscle area at $\mathrm{C} 3 * 0.538+1.111$ fitted the data well $\left(R^{2}=0.80\right)$. The multivariable formula total skeletal muscle volume $=-23.607+$ $0.270 *$ area $\mathrm{C} 3+16.234 *$ height (meters) $+0.060 *$ weight (kilogram) $+3.129 *$ sex (female $=1$, male $=2$ ) $-0.022 *$ age (years) explained $88 \%$ of the variance in the data.

Conclusion Skeletal muscle area on a single MRI slice of the head and neck region can be used to estimate total skeletal muscle volume in adults.

\section{Introduction}

In recent years, it has become apparent that malnutrition or sarcopenia has profound influence on treatment outcome and survival in patients with a variety of illnesses. ${ }^{1,2}$ Specifically in cancer patients, a low skeletal muscle mass (SMM), sometimes termed sarcopenia has been associated with higher incidence of chemotherapy related toxicity, postoperative complications, longer hospital stay and lower disease free and overall survival. ${ }^{3-6}$ This relationship has been shown in breast, colorectal, hepato-pancreatico-biliary, renal and lung cancer, amongst others. ${ }^{7-9}$ Magnetic resonance imaging (MRI) and computed tomography (CT) imaging are two highly accurate imaging modalities used for the assessment of SMM. Whole-body MRI is considered to be the gold standard for SMM assessment, but is also costly and time-consuming to perform and to assess. ${ }^{10,11}$

The most commonly used method for the measurement of SMM in cancer patients is based on CT imaging: skeletal muscle area (SMA) is measured on a single transversal slice at the level of the third lumbar vertebra (L3), as a proxy for wholebody SMM. ${ }^{7,12}$ This method has been validated using whole-body MRI; it has been shown that SMA on a single transversal slice at the level of $L 3$ is strongly correlated with total skeletal muscle (SM) volume in healthy volunteers as measured using whole-body MRI. ${ }^{13,14}$ Abdominal CT imaging is frequently routinely performed in patients with certain cancer types during diagnostic work-up and follow-up allowing for routine evaluation of SMM in these patients without the burden and costs of additional diagnostics.

However, CT imaging of the abdomen is not routinely performed in head and neck cancer (HNC) patients. It is known that risk factors for developing sarcopenia, such as malnutrition and chronic inflammation, are highly prevalent in HNC patients. ${ }^{15}$ Also, adverse outcomes associated with low SMM such as chemotherapy related toxicity and postoperative complications, occur frequently. ${ }^{16}$ Head and neck CT imaging or MRI is routinely performed in the diagnostic work-up and follow-up of head and neck cancer patients. ${ }^{17}$ An alternative SMM measurement method was published using SMA at the level of the third cervical vertebra (C3), which is featured on CT imaging of the head and neck area. ${ }^{18} \mathrm{~A}$ strong correlation between SMA at the level of C3 and L3 $(r=0.785)$ was found. Using a multivariable prediction equation, correlation between measured SMA at L3 and estimated SM area at L3 from C3 was excellent $(r=0.895)$. However, SMA at C3 was not yet compared to total body SMM as measured on whole-body MRI, and other cervical levels were not evaluated. 
The objective of this study was to establish the correlation between SMA at the level of C3, C4 and C5 and total SM volume as measured using whole-body MRI, and to formulate a prediction equation to estimate total SM volume from a single slice SMA in the head and neck region. This study was performed in accordance with the STROBE checklist.

\section{Methods}

This investigation was an additional analysis of data ascertained between 2005 and 2012 at the Institute of Human Nutrition and Food Science at the University of Kiel (Christian-Albrechts-University, Kiel, Germany). ${ }^{13}$ In this study, MRI data of 142 healthy Caucasians ( 75 women and 67 men) aged 19-65 years with BMI (in $\mathrm{kg} / \mathrm{m}^{2}$ ) from 20.0 to 47.7 were collected. All subjects were recruited from the loca community, by using local advertisement and notice-board postings. Exclusion criteria were smoking, metallic implants, pregnancy, acute or chronic diseases, and intake of medication that influences body composition. Each participant was informed about the objectives and methods of the study and asked to sign an informed consent. All protocols were approved by the ethical committee of the Christian-Albrechts-University and were performed in accordance with the Declaration of Helsinki. Measurements of body composition took place at the Institute of Human Nutrition and Food Science, Christian-Albrechts-University, Kiel (anthropometric measures) and the Clinic for Diagnostic Radiology, Universitätsklinikum Schleswig-Holstein (MRI), Germany.

\section{Anthropometric measurements}

Body weight was measured to the nearest $0.01 \mathrm{~kg}$ with an electronic scale coupled to the BOD POD device (TANITA, Japan). Height was measured using a seca stadiometer (seca, Hamburg, Germany) with an accuracy of $0.5 \mathrm{~cm}$.

\section{Magnetic resonance imaging}

Body composition of all subjects was measured using whole-body MRI. Standard protocols were described in more detail elsewhere. ${ }^{19}$ In short, imaging of SMM was obtained using a 1.5-T Magnetom Avanto Vision Scanner (Siemens Medica Systems, Erlangen, Germany), using a T1-weighted-gradient echo sequence (repetition time: $157 \mathrm{~ms}$; echo time: $4 \mathrm{~ms}$ ). Transversal scans were obtained from the wrist to ankle with a slice thickness of $8 \mathrm{~mm}$ and a $2 \mathrm{~mm}$ interslice gap. Subjects were scanned in a supine position with arms extended above the head. During the measurement of abdominal and thoracic regions, subjects were asked to hold their breath. All SM volumes were manually segmented by a single researcher (L.S.) using commercially available medical image segmentation software (SliceOmatic v4.3, Tomovision, Canada).

Total SM volume was calculated by adding up SM volume of the trunk and the upper and lower extremities. Upper extremities were defined as from the wrists to humerus heads, and lower extremities were defined as from the femoral heads to the ankles. The trunk was segmented between the humerus and femora heads. Later three single slices at the level of C3, C4 and C5 were additionally segmented in all participants by a different researcher (S.B.) using SliceOmatic v5.0 segmentation software, following a previously formulated method. ${ }^{18}$ The SMA at C3, C4 and C5 was defined as the SM area of the paravertebral muscles (PVM) and both sternocleidomastoid muscles (SCM), identical to the previously published method. ${ }^{18}$

\section{Statistical analysis}

All statistical analyses were performed using the IBM SPSS Statistics version 25.0 software package (Chicago, Illinois, USA). Results were considered statistically significant if the $p$-value was less than 0.05 . A test for normality (KolmogorovSmirnoff test) and histograms were used to assess whether continuous variables were normally distributed. Continuous data are represented as mean \pm standard deviation (SD) if normally distributed, and median \pm interquartile range (IQR) if skewed. Categorical data are represented as a number and percentage of the total. Pearson's or Spearman's correlation coefficients were calculated to evaluate bivariate linear correlation between single slice SMA and total SM volume. Differences between correlation coefficients of slice SMA and total SM volume were tested by using the method of Steiger and Fisher's $\mathrm{Z}$ transformation. ${ }^{20} \mathrm{~A}$ simple linear regression model was used to create a prediction equation from single slice SMA for total SM volume. Multivariable linear regression with a crossvalidation step was used to create a multivariable prediction formula for total SM volume from single slice SMA. A cross-validation dataset was created by randomly dividing the total subject dataset 10 times into a 'training' and a 'test' equal datasets, as well as an eleventh "validation 1 and 2" dataset. Multivariable prediction equation formulation was carried out using backward selection and a $p$-value of 0.05 . Bland-Altman plots were constructed to assess agreement between measurements. ${ }^{21}$ 


\section{Results}

For this study, 142 healthy subjects who had undergone whole-body MRI were evaluated for inclusion. Two male subjects were excluded because of major movement artefacts in the head and neck region, impeding accurate identification and segmentation of SMA at the level of C3, C4 and C5. In total, 140 subjects were included for analysis. Subjects' characteristics are summarized in Table 1. Men and women differed significantly in height, weight and total SM volume. Most continuous variables were normally distributed. Age and BMI were not normally distributed.

\section{Single slice SMA and total SM volume}

Mean results for single slice SMA and total SM volume are summarized in Table 1. All single slice SMA in the head and neck region correlated significantly with total SM volume $(p<0.01)$. There was not one single slice SMA significantly stronger correlated with total SM volume (Fisher's Z transformation and method of Steiger: $p>0.05)$. However, because the correlation coefficient between single slice SMA at C3 and total SM volume was highest $(r=0.892)$, compared to C4 $(r=0.886)$ and C5 $(r=0.876)$, the single slice SMA at C3 was chosen as the reference slice for establishing a prediction formula.

\section{Table 1: Characteristics of study subjects}

\begin{tabular}{|c|c|c|c|}
\hline & Total $(n=140)$ & Women $(n=75)$ & $\operatorname{Men}(n=65)$ \\
\hline Age, $y^{+}$ & $37.0[28.0-46.75]$ & $35.0[29.0-48.0]$ & $39.0[27.0-46.0]$ \\
\hline 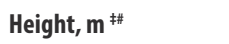 & $1.75 \pm 0.08$ & $1.69 \pm 0.07$ & $1.81 \pm 0.05$ \\
\hline 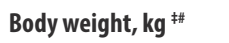 & $82.5 \pm 19.7$ & $77.4 \pm 22.0$ & $88.3 \pm 14.9$ \\
\hline $\begin{array}{l}\text { BMI, } \mathbf{k g} / \mathrm{m}^{2 \dagger} \\
\text { Normal weight } \% \\
\text { Overweight } \% \\
\text { Obese } \%\end{array}$ & $\begin{array}{c}25.2[22.8-30.1] \\
47.9 \\
25.7 \\
26.4\end{array}$ & $\begin{array}{c}24.4[22.1-31.1] \\
54.7 \\
17.3 \\
28.0\end{array}$ & $\begin{array}{c}26.1[23.2-29.9] \\
40.0 \\
35.4 \\
24.6\end{array}$ \\
\hline $\begin{array}{l}\text { Single slice SMA }\left(\mathrm{cm}^{2}\right)^{\ddagger} \\
\text { C3 } \\
\text { C4 } \\
\text { C5 } \\
\text { L3 }\end{array}$ & $\begin{array}{l}46.0 \pm 10.4 \\
47.9 \pm 11.1 \\
53.3 \pm 11.8 \\
152.8 \pm 38.9\end{array}$ & $\begin{array}{c}38.4 \pm 5.7 \\
39.7 \pm 6.1 \\
44.7 \pm 6.6 \\
123.7 \pm 20.8\end{array}$ & $\begin{array}{c}54.9 \pm 7.0 \\
57.2 \pm 7.5 \\
63.2 \pm 8.1 \\
186.4 \pm 25.4\end{array}$ \\
\hline Total SM volume $(\mathrm{L})^{\neq \#}$ & $25.9 \pm 6.3$ & $21.2 \pm 3.5$ & $31.3 \pm 4.0$ \\
\hline
\end{tabular}

Abbreviations: SM - skeletal muscle; C3 - third cervical vertebra; C4 - fourth cervical vertebra; C5 fifth cervical vertebra; $L 3$ - third lumbar vertebra.

${ }^{\dagger}$ Median [IQR].

${ }^{\ddagger}$ Mean \pm SD.

"Significant difference between men and women, $\mathrm{p}<0.05$ (unpaired $t$-test or Mann-Whitney $\mathrm{U}$ test).
Using linear regression, the following univariable prediction equation 1 for total SM volume was established from single slice SMA at C3.

\section{Univariable prediction equation 1:}

Total SM volume $(\mathrm{L})=\mathrm{SM}$ area at $\mathrm{C} 3 * 0.538+1.111$

$\mathrm{R}^{2}=0.795$, standard error of the estimate $(\mathrm{SEE})=2.84 \mathrm{~L}$

The relationship and agreement between measured total SM volume and estimated total SM volume using prediction equation 1 is shown in Figure 1. Estimation of total SM volume using the univariable prediction equation 1 is possible. The Bland-Altman plot shows few major outliers both below and above the \pm 1.96 SD references lines.

Figure 1. Estimated and measured SM volume using univariable prediction formula 1

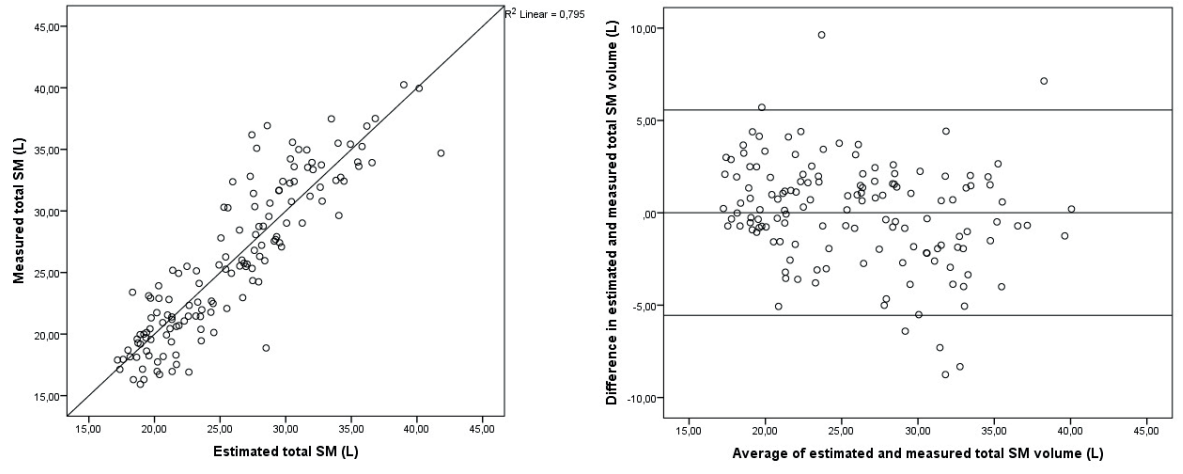

Figure 1: Correlation between estimated total SM volume based on SMA at the level of $C 3$ and measured total SM volume in liters. Estimated total SM in liters was calculated using univariable prediction equation 1 . 


\section{Cross-validation and multivariable prediction equation}

establishment for total SM volume

A 10-fold cross-validation step was performed. Variables entered into the multivariable linear regression model, apart from single slice SMA at C3, were height $(\mathrm{m})$, weight $(\mathrm{kg})$, sex and age $(\mathrm{y})$, because these variables were significantly associated with single slice SMA at C3 in univariable linear regression analysis. Sex was coded " 1 " for female subjects and " 2 " for male subjects. The following average prediction equations were created in the 10 'training' and 'test' datasets: 2 and 3 .

\section{'Training' multivariable prediction equation 2:}

Total SM volume $(L)=-24.795+0.289 *$ SMA at $C 3+16.823 *$ Height $(m)+0.059 *$ Weight $(\mathrm{kg})+2.598 *$ Sex $-0.018 *$ Age $(\mathrm{y})$

$\mathrm{R}^{2}=0.878, \mathrm{SEE}=2.19 \mathrm{~L}$

\section{'Test' multivariable prediction equation 3:}

Total SM volume $(\mathrm{L})=-22.416+0.251 * \mathrm{SMA}$ at $\mathrm{C} 3+15.685 *$ Height $(\mathrm{m})+0.061 *$ Weight $(\mathrm{kg})+3.680 *$ Sex $-0.026 *$ Age $(\mathrm{y})$

$\mathrm{R}^{2}=0.878, \mathrm{SEE}=2.19 \mathrm{~L}$

Prediction equations 2 and 3 were tested in validation datasets 1 and 2 to estimate total SM volume; both prediction formulas showed excellent correlation with total SM volume in both datasets. Figure $\mathbf{2}$ shows the agreement between measured total SM volume and estimated total SM volume using prediction equations 2 and 3 in Bland-Altman plots. Both plots show good agreement between measured total SM volume and estimated total SM volume, with about $5 \%$ of measurements outside the $95 \%$ confidence intervals.

Based on these results, we combined prediction equation 2 and prediction equation 3 into the following final multivariable prediction equation 4:

\section{Multivariable prediction equation 4:}

Total SM volume $(\mathrm{L})=-23,607+0,270 *$ SMA at $\mathrm{C} 3+16.234 *$ Height $(\mathrm{m})+0.060 *$ Weight $(\mathrm{kg})+3.129 *$ Sex $-0.022 *$ Age $(\mathrm{y})$

$\mathrm{R}^{2}=0.879, \mathrm{SEE}=2.18 \mathrm{~L}$

Figure 3 shows the direct relationship between measured total SM volume and estimated total SM volume based on single slice SMA at C3 using multivariable prediction equation 4 in a scatter plot, and the agreement between measured total SM volume and estimated total SM volume using prediction equation 4 in a
Bland-Altman plot. There is good agreement between measured total SM volume and estimated total SM volume, with $5 \%$ of measurements outside of \pm 1.96 SD references lines. There was 1 major outlier, where actual and estimated total SM volume differed more than $3 \mathrm{SD}$. This particular subject was an extraordinary muscular woman

Figure 2: Bland-Altman plots illustrating agreement between estimated and measured total
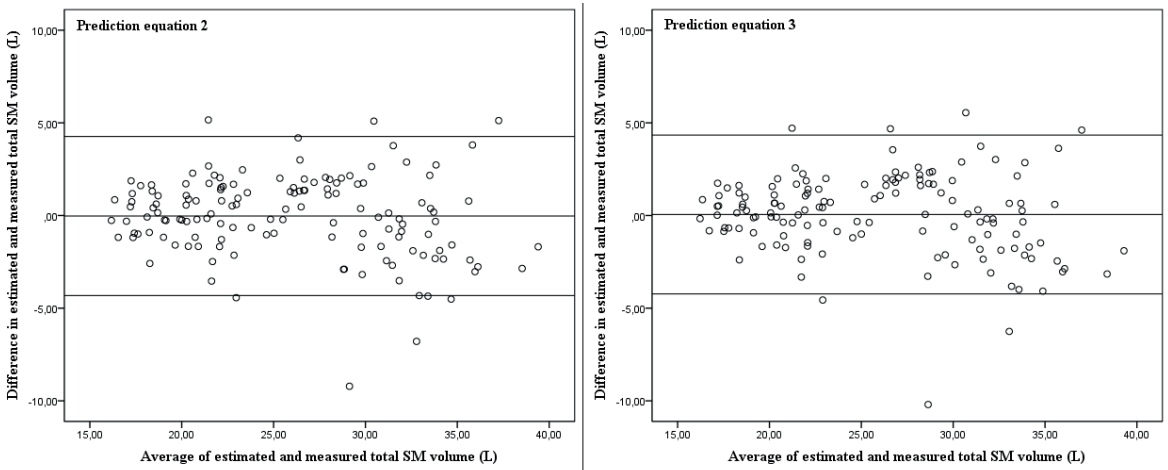

Figure 2: Bland-Altman plots illustrating the agreement between estimated SM volume in liters using multivariable prediction equations 2 and 3 using SM area measurement on $C T$ at the level of $\mathrm{C} 3$, and measured total SM volume in liters.

\section{Figure 3: Estimation of total SM volume from CSA at the level of C3}

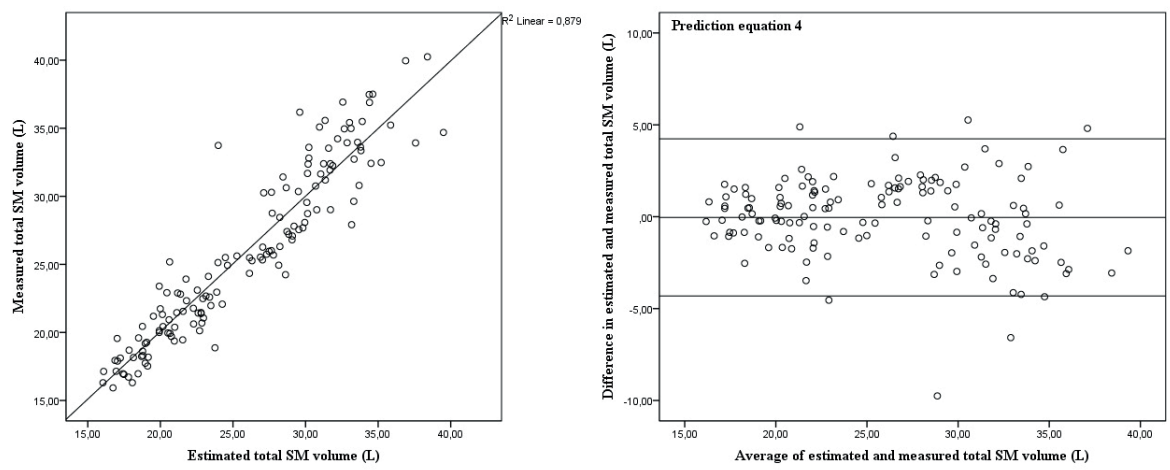

Figure 3: Scatter plot and Bland-Altman plot illustrating the agreement between estimated and measured total SM volume from CSA at the level of $\mathrm{C} 3$ using multivariable prediction formula 4. 


\section{Discussion}

Previous research has shown that it is possible to accurately estimate total SM volume from a single transversal MRI slice at the level of $L 3$. This study shows that total SM volume can also be accurately estimated from a transversal MRI slice at the level of $\mathrm{C} 3$ in healthy volunteers. Using a multivariable formula including SMA at the level of $\mathrm{C} 3$, height, weight, sex and age of the subject, estimation of total SM volume is even more precise. We showed that the C3 measurement method can be used reliably to investigate body composition in subjects with imaging of the head and neck region only.

In recent years, the radiological assessment of SMM has been widely adopted to investigate body composition and outcome in, amongst others, cancer patients. Low SMM is a recognized predictive and prognostic marker for adverse outcomes in a variety of cancer types. ${ }^{22-24}$ Several treatment interventions are investigated; such as treatment adaptation based on the body composition of an individual patient, or physical and nutritional rehabilitation prior to treatment. ${ }^{25,26}$ Patients with HNC often present with signs of malnutrition, and as such are at risk of having low SMM prior to start of treatment. Adverse outcomes associated with low SMM, such as chemotherapy related toxicity and wound healing problems, are highly prevalent in HNC patients.

In the last 5 years, several studies have reported on the incidence and relevance of low SMM in HNC patients using a measurement of SMM at the level of C3 on head and neck CT imaging. The prevalence of low SMM was high in HNC patients, ranging from $30 \%$ up to more than $80 \%$ in elderly HNC patients. ${ }^{27}$ In HNC patients, low SMM as measured at the level of $\mathrm{C} 3$ has been associated with increased incidence of postoperative complications, chemotherapy (dose-limiting) toxicity and decreased survival. ${ }^{28-32}$ It is also predictive of frailty in HNC patients. ${ }^{33,34} \mathrm{~A}$ noted advantage in HNC patients of measurement of SMM at the level of C3 is that it can be assessed on routinely performed imaging, thus without any additional costs or burden for the patient. In the future, HNC patients at high risk of adverse outcomes related to sarcopenia may benefit from additional supportive treatment or individualized primary treatment. Several possible interventions may be considered, such as intensive physical and nutritional prehabilitation, and treatment adaptation such as modification of surgical treatment and alternative chemotherapy dosing based on the body composition of a patient.
Some limitations to this study need to be addressed. This study was performed in healthy subjects, whose average body composition may differ from the average HNC patient's body composition. There were no underweight subjects analyzed in this study, while it is known that some HNC patients are underweight at diagnosis. Patients with HNC are generally aged 45 years or older, while the median age in this cohort of healthy volunteers was 37 years. Moreover, volunteers who smoked were excluded from participation in this study, while the majority of HNC patients uses or has used tobacco. However, there is no apparent reason why the relationship between SMA at C3 and total SM volume should be significantly different in smokers, elderly or HNC patients than in non-smoking, healthy individuals. In a previous study using CT we found no difference in the relation of C3 and L3 muscle CSA measurements between trauma patients (considered otherwise-healthy controls) and HNC patients. ${ }^{18}$

Also, it should be noted that the development of a second primary tumor is relatively common in tobacco- and alcohol-associated HNC, with up to 20 percent of patients developing another tumor in the same region. As a result, some patients diagnosed with HNC will have received prior treatment for a different HNC in the same region, for example with radiotherapy or surgery. It is unclear what effect prior radiotherapy has on the SMA and skeletal muscle quality. This issue still needs to be addressed. Lastly, in this study only MRI has been used to assess the relationship between SM area in the head and neck region and total SM volume. In clinical practice many patients will undergo CT imaging in the diagnostic process instead of MRI. Recent studies in HNC patients compared a measurement of SMA on CT and MRI, and found that differences in SMA measured by $C T$ and $M R I$ are negligible. ${ }^{35,36}$

Several different cut-off values for low SMM have been published; most are in part dependent of its outcome value and reference population. This hinders generalization of SM measurements and its association with clinical outcomes. ${ }^{37}$ This study provides valuable information on the relationship between SMA at the level of C3 and total SM volume, but it does not provide specific cut-off values for low SMM or low SM volume based on SMA at the level of C3.

\section{Conclusion}

The skeletal muscle area on a single MRI slice at the level of C3 is strongly correlated with total skeletal muscle volume as measured on whole-body MRI. Via a multivariable prediction formula, total skeletal muscle volume can be accurately predicted from skeletal muscle area at the level of C3 in healthy adults. The 
skeletal muscle area at the level of $\mathrm{C} 3$ can be used as an alternative to that of $\mathrm{L} 3$ to assess total skeletal muscle volume in patients who have only undergone imaging of the head and neck area.

\section{Acknowledgements}

$\mathrm{SB}, \mathrm{PdJ}$ and $\mathrm{RdB}$ would like to thank the Institute of Human Nutrition and Food Science at the University of Kiel for offering the opportunity to collaborate on this research.

\section{References}

1. Prado CM, Cushen SJ, Orsso CE, Ryan AM. Sarcopenia and cachexia in the era of obesity: clinica and nutritional impact. Proc Nutr Soc. 2016;75(2):188-198.

2. Cruz-Jentoft AJ, Bahat G, Bauer J, et al. Sarcopenia: revised European consensus on definition and diagnosis. Age Ageing. 2019;48(1):16-31.

3. Rier HN, Jager A, Sleiffer S, Maier AB, Levin M. The Prevalence and Prognostic Value of Low Muscle Mass in Cancer Patients: A Review of the Literature. Oncologist. 2016;21(11):1396-1409. Levolger S, van Vugt JLA, de Bruin RWF, IJzermans JNM. Systematic review of sarcopenia in patients operated on for gastrointestinal and hepatopancreatobiliary malignancies. Br J Surg. 2015;102(12):1448-1458.

5. Martin L, Birdsell L, MacDonald N, et al. Cancer Cachexia in the Age of Obesity: Skeletal Muscle Depletion Is a Powerful Prognostic Factor, Independent of Body Mass Index. J Clin Oncol. 2013;31(12):1539-1547.

6. Shachar SS, Williams GR, Muss HB, Nishijima TF. Prognostic value of sarcopenia in adults with solid tumours: A meta-analysis and systematic review. Eur J Cancer. 2016;57:58-67.

7. Prado $C M$, Lieffers JR, McCargar LJ, et al. Prevalence and clinical implications of sarcopenic obesity in patients with solid tumours of the respiratory and gastrointestinal tracts: population-based study. Lancet Oncol. 2008;9(7):629-635.

8. Sjøblom B, Grønberg BH, Benth $\mathrm{J} \rrbracket$, et al. Low muscle mass is associated with chemotherapyinduced haematological toxicity in advanced non-small cell lung cancer. Lung Cancer. 2015;90(1):85-91.

9. Cushen SJ, Power DG, Teo MY, et al. Body composition by computed tomography as a predictor of toxicity in patients with renal cell carcinoma treated with sunitinib. Am J Clin Oncol Cancer Clin Trials. 2017;40(1):47-52.

10. Mitsiopoulos N, Baumgartner RN, Heymsfield SB, Lyons W, Gallagher D, Ross R. Cadaver validation of skeletal muscle measurement by magnetic resonance imaging and computerized tomography. J Appl Physiol. 1998;85(1):115-122.

11. Heymsfield SB, Adamek M, Gonzalez MC, Jia G, Thomas DM. Assessing skeletal muscle mass: historical overview and state of the art. J Cachexia Sarcopenia Muscle. 2014;5(1):9-18.

12. Shen W, Punyanitya $M$, Wang Z, et al. Total body skeletal muscle and adipose tissue volumes: estimation from a single abdominal cross-sectional image. J Appl Physiol. 2004:97(6):23332338.

13. Schweitzer L, Geisler C, Pourhassan $M$, et al. What is the best reference site for a single MRI slice to assess whole-body skeletal muscle and adipose tissue volumes in healthy adults?1. Am J Clin Nutr. 2015;102(1):58-65.

14. Schweitzer L Geisler C Pourhassan M, et al. Estimation of Skeletal Muscle Mass and Viscera Adipose Tissue Volume by a Single Magnetic Resonance Imaging Slice in Healthy Elderly Adults. J Nutr. 2016;146(10):2143-2148.

15. Hébuterne X Lemarié E, Michallet M de Montreuil CB, Schneider SM Goldwasser F. Prevalence of Malnutrition and Current Use of Nutrition Support in Patients With Cancer. J Parenter Enter Nutr. 2014;38(2):196-204. doi:10.1177/0148607113502674

16. Hasan Z, Dwivedi RC, Gunaratne DA, Virk SA, Palme CE, Riffat F. Systematic review and metaanalysis of the complications of salvage total laryngectomy. Eur J Surg Oncol. 2017;43(1):42-51.

17. Grégoire V, Lefebvre J-L, Licitra L, Felip E. Squamous cell carcinoma of the head and neck: EHNS-ESMO-ESTRO Clinical Practice Guidelines for diagnosis, treatment and follow-up. Ann Oncol. 2010;21:v184-v186.

18. Swartz JE, Pothen AJ, Wegner I, et al. Feasibility of using head and neck CT imaging to assess skeletal muscle mass in head and neck cancer patients. Oral Oncol. 2016;62:28-33.

19. Bosy-Westphal A, Kossel E, Goele K, et al. Contribution of individual organ mass loss to weight loss-associated decline in resting energy expenditure. Am J Clin Nutr. 2009;90(4):993-1001.

20. Steiger J. Tests for Comparing Elements of a Correlation Matrix. Psychol Bull. 1980;87:245-251

21. Martin Bland J, Altman DG. Statistical Methods for Assessing Agreement Between Two Methods of Clinical Measurement Lancet 1986:327(8476):307-310. 
22. Huiskamp LFJ, Chargi N, Devriese LA, May AM, Huitema ADR, de Bree R. The Predictive Value of Low Skeletal Muscle Mass Assessed on Cross-Sectional Imaging for Anti-Cancer Drug Toxicity: .

23. Su H, Ruan J, Chen T, Lin E, Shi L. CT-assessed sarcopenia is a predictive factor for both longterm and short-term outcomes in gastrointestinal oncology patients: a systematic review and AuPC-M,LiH-L Le

24. Au PC-M, Li H-L, Lee GK-Y, et al. Sarcopenia and mortality in cancer: A meta-analysis. Osteoporos Sarcopenia. 2021, $7($ Suppl 1):S28-S33.

25. Moug SJ, Barry SJE, Maguire $\mathrm{S}$, et al. Does prehabilitation modify muscle mass in patients with rectal cancer undergoing neoadjuvant therapy? A subanalysis from the REx randomised

26. Roche M, Ravot C, Malapert A, et al. Feasibility of a prehabilitation programme dedicated to older patients with cancer before complex medical-surgical procedures: The PROADAPT pilot study protocol. BMJ Open. 2021;11(4).

27. Chargi N, Bril Sl, Emmelot-Vonk MH, de Bree R. Sarcopenia is a prognostic factor for overal survival in elderly patients with head-and-neck cancer. Eur Arch Oto-Rhino-Laryngology. 2019;276(5):1475-1486.

28. Wendrich AW, Swartz JE, Bril Sl, et al. Low skeletal muscle mass is a predictive factor for chemotherapy dose-limiting toxicity in patients with locally advanced head and neck cancer. Oral Oncol. 2017;71:26-33.

29. Bril SI, Pezier TF, Tijink BM, Janssen LM, Braunius WW, Bree R. Preoperative low skeletal muscle mass as a risk factor for pharyngocutaneous fistula and decreased overall survival in patients undergoing total laryngectomy. Head Neck. 2019;41(6):1745-1755.

30. Ansari E, Chargi N, van Gemert JTM, et al. Low skeletal muscle mass is a strong predictive factor for surgical complications and a prognostic factor in oral cancer patients undergoing mandibular reconstruction with a free fibula flap. Oral Oncol. 2020;101:104530.

31. Achim V, Bash J, Mowery A, et al. Prognostic Indication of Sarcopenia for Wound Complication After Total Laryngectomy. JAMA Otolaryngol Neck Surg. 2017;143(12):1159.

32. Wong A, Zhu D, Kraus D, Tham T. Radiologically Defined Sarcopenia Affects Survival in Head and Neck Cancer: A Meta-Analysis. Laryngoscope. 2021;131(2):333-341.

33. Zwart AT, Hoorn A, Ooijen PMA, Steenbakkers RJHM, Bock GH, Halmos GB. CT-measured skeletal muscle mass used to assess frailty in patients with head and neck cancer. J Cachexia Sarcopenia Muscle. 2019;10(5):1060-1069.

34. Meerkerk CDA, Chargi N, de Jong PA, van den Bos F, de Bree R. Sarcopenia measured with handgrip strength and skeletal muscle mass to assess frailty in older patients with head and neck cancer. J Geriatr Oncol. 2021;12(3):434-440.

35. Chargi N, Ansari E, Huiskamp LFJ, Bol G, de Bree R. Agreement between skeletal muscle mass measurements using computed tomography imaging and magnetic resonance imaging in head and neck cancer patients. Oral Oncol. 2019;99:104341.

36. Zwart AT, Becker J-N, Lamers MJ, et al. Skeletal muscle mass and sarcopenia can be determined with 1.5-T and 3-T neck MRI scans, in the event that no neck CT scan is performed. Eur Radiol. 2021;31(6):4053-4062.

37. Walowski CO, Braun W, Maisch MJ, Jensen B, Peine S, Norman K, et al. Reference values for skeletal muscle mass - current concepts and methodological considerations. Nutrients 2020;12:1-36. 
Part III

Radiological assessment of body composition and outcomes in head and neck cancer patients 


\section{Chapter 5}

Pretreatment low skeletal muscle mass is associated with chemotherapy dose-limiting toxicity in head and neck cancer patients undergoing primary chemoradiotherapy with high dose cisplatin

Sandra I. Bril, Abrahim Al-Mamgani, Najiba Chargi, Peter Remeijer, Lot A. Devriese, Jan Paul de Boer, Remco de Bree 


\section{Abstract}

Background Low skeletal muscle mass (SMM) is an adverse prognostic facto for chemotherapy dose-limiting toxicity (CLDT). In patients with locallyadvanced-head-and-neck-squamous-cell-carcinoma (HNSCC) undergoing chemoradiotherapy (CRT), low SMM is a predictor for CDLT. We aimed to validate these findings.

Methods Consecutive LA-HNSCC patients treated with primary CRT with highdose cisplatin were retrospectively included. SMM was measured on pre-treatmen CT-imaging. A cumulative cisplatin dose below $200 \mathrm{mg} / \mathrm{m}^{2}$ was defined as CDLT.

Results 153 patients were included; 37 (24.2\%) experienced CDLT and 84 had low SMM (54.9\%). Patients with low SMM experienced more CDLT than patients with normal SMM (35.7\% vs $10.1 \%$, p < 0.01). Low SMM (OR 3.99 [95\%Cl 1.56-10.23] $\mathrm{p}=0.01)$ and an eGFR of $60-70 \mathrm{~mL} / \mathrm{min}(\mathrm{OR} 5.40$ [95\%Cl 1.57-18.65], $\mathrm{p}<0.01)$ were predictors for CDLT.

Conclusion Pre-treatment low SMM is associated with CDLT in LA-HNSCC patients treated with primary CRT. Routine SMM assessment may allow for CDLT risk assessment and treatment optimalization.

\section{Introduction}

Locally advanced head and neck squamous cell carcinoma (LA-HNSCC) is preferably treated with concomitant chemoradiotherapy (CRT) with cisplatin, with or without prior surgery. ${ }^{1}$ The standard-of-care cisplatin regimen consists of three three-weekly courses of high dose cisplatin at a dose of $100 \mathrm{mg} / \mathrm{m}^{2}$ body surface area (BSA), with a cumulative dose of $300 \mathrm{mg} / \mathrm{m} 2$ BSA cisplatin. ${ }^{2}$ The addition of high dose cisplatin chemotherapy to radiotherapy treatment improves locoregional disease control and results in a $6.5 \%$ increase in 5-year overall survival. ${ }^{3}$ Large prospective trials and retrospective studies show that a higher cumulative dose is associated with better survival rates. ${ }^{4-7}$

The addition of cisplatin also results in a significant increase in the toxicity of treatment, such as acute nephrotoxicity, bone marrow depression or severe nausea and vomiting, which cause treatment delay, dose reduction and treatment cessation as well as decreased quality of life.,

Approximately $30 \%$ of patients experience chemotherapy dose-limiting toxicity (CDLT) and are unable to complete full treatment. ${ }^{9}$ There are severa contraindications for the use of high dose cisplatin, such as a decreased rena function, severe hearing loss and poor WHO functional status. Nevertheless, even in absence of these contra-indications, still 30\% of patients experience CDLT in daily clinically practice which currently cannot be identified in advance. Therefore there is a clinical need for additional predictive characteristics or biomarkers to accurately identify LA-HNSCC patients at high risk of CDLT from cisplatin.

In recent years, radiologically identified sarcopenia or low skeletal muscle mass (SMM) has been identified as a novel predictive and prognostic factor in cancer patients. Pre-treatment low SMM is associated with chemotherapy induced toxicity and CDLT in patients with a variety of cancer types, including lung, renal cell, colorectal and breast cancer. ${ }^{10,11}$ Several risk factors for low SMM are known including malnutrition, immobilization and chronic illnesses including cancer. ${ }^{12}$ In HNSCC, malnutrition at diagnosis is very common, and several retrospective studies report an incidence of approximately 50\% of low SMM in HNSCC patients. ${ }^{9,13-15}$

Recent retrospective studies in LA-HNSCC patients also concluded that pretreatment low SMM was a significant predictor of CDLT in patients treated with CRT with platinum-based chemotherapy., ${ }^{9,16}$ The purpose of this study was to 
investigate and validate the predictive value of low SMM on CDLT in a larger cohort of LA-HNSCC patients, treated with standard-of-care treatment with primary CRT with high dose cisplatin.

\section{Methods}

This study was performed as a secondary analysis of a prior retrospective study. ${ }^{6}$ All data were used in an coded fashion. Because of the retrospective nature of this study, formal informed consent or medical ethical approval was waived at the time of the inception of this study. This research was conducted in accordance with the Declaration of Helsinki and all subsequent legislation.

\section{Patient and study design}

All patients were treated at the Netherlands Cancer Institute in Amsterdam, The Netherlands, with curative intent. Between January 2008 and December 2015 , all 279 consecutive patients with histologically proven squamous cell carcinoma of the oropharynx, hypopharynx, or larynx who were eligible for concomitant primary chemoradiotherapy with three three-weekly courses of high dose cisplatin courses at $100 \mathrm{mg} / \mathrm{m}^{2}$ BSA were identified. Patients who were not treated with cisplatin for any reason, and patients who received cisplatin in anothe regimen such as weekly cisplatin or carboplatin were excluded. Patients without recent CT or MRI scans (less than 3 months) of the head and neck area prior to TL were excluded. Patients who had severe dental artifacts at the level of C3 that impeded accurate assessment of SMM were also excluded.

Relevant clinical information such as weight, stature, body mass index (BMI), smoking, AJCC stage according to the $7^{\text {th }}$ AJCC staging manual and outcome data were retrieved from medical records. The Adult Comorbidity Evaluation index (ACE27) was used to measure comorbidities. ${ }^{17}$ In oropharyngeal cancer, HPV status was assessed by p 16 staining, followed by high-risk HPV PCR for confirmation. Survival data were collected until February 2017. Because of a known better prognosis of HPV-related oropharyngeal cancer, those patients were excluded from survival analysis.

\section{Chemotherapy dose-limiting toxicity}

Chemotherapy dose-limiting toxicity was defined as any toxicity resulting in a cumulative cisplatin dose of less than $200 \mathrm{mg} / \mathrm{m}^{2}$. This could be because of a chemotherapy dose-reduction of $\geq 50 \%$ (e.g. due to neutropenia or nephrotoxicity) after the first cycle of treatment, a postponement of treatment of $\geq 4$ days (e.g. in the case of bone marrow suppression) resulting in termination of a cycle combined with a dose-reduction, or a definite termination of chemotherapy after the first cycle of therapy. The aim was to complete all three cycles, but if treatment tolerance was perceived to be low, two full cycles of high dose cisplatin was accepted as adequate treatment.

\section{CT image acquisition}

As part of radiotherapy planning, pre-treatment head and neck CT-imaging in radiation mould was performed in all patients. Patients were immobilized in supine treatment position in a custom-made head-and-neck mask. For planning, contrast-enhanced 3-mm slides CT-scan simulation was performed in all patients. All patients were treated with intensity-modulated radiotherapy (IMRT) or volumetric modulate arc therapy (VMAT). The radiation treatment consisted of 46 Gy of elective irradiation to both sides of the neck (level II-IV in case of nodenegative neck and level II-V in case of cervical lymph node metastases), followed by a boost of $24 \mathrm{~Gy}$ in 12 fractions to the primary tumor and the involved nodes in case of node-positive disease, to a total dose $70 \mathrm{~Gy}$.

\section{Image evaluation}

Measurement of SMM was performed at the level of C3 according to a method previously described by Swartz et al. ${ }^{18}$ In brief, a single axial CT-slide at level C3 was selected using a standard procedure: the first slide to completely show the entire vertebral arc when scrolling through the C3 vertebra from caudal to cephalic direction was selected. Skeletal muscle tissue was identified using Hounsfield unit $(\mathrm{HU})$ ranges settings from -29 to $+150 \mathrm{HU}$, to avoid overestimation of skeletal muscle area and to exclude fatty tissue (which has a $\mathrm{HU}$ value below -30). ${ }^{19}$ The outer contours of the sternocleidomastoid and paravertebral muscles were traced manually, as is shown in Figure 1, using the Worldmatch Research Software Package, an in-house software package designed for image evaluation, registration and delineation for radiotherapy. The cross-sectional muscle area (CSMA) at the level of C3 was calculated as the sum of the delineated areas of the paravertebral muscles and both sternocleidomastoid muscles within $\mathrm{HU}$ ranges of -29 to +150 in $\mathrm{cm}^{2}$. All CT slides were analyzed by a single researcher (S.B.). The CSMA at the level of C3 was then normalized for stature to calculate a cervica skeletal muscle index (CSMI). ${ }^{20}$ 
Figure 1: Skeletal muscle area segmentation at the level of C3 using the WorldMatch software program.

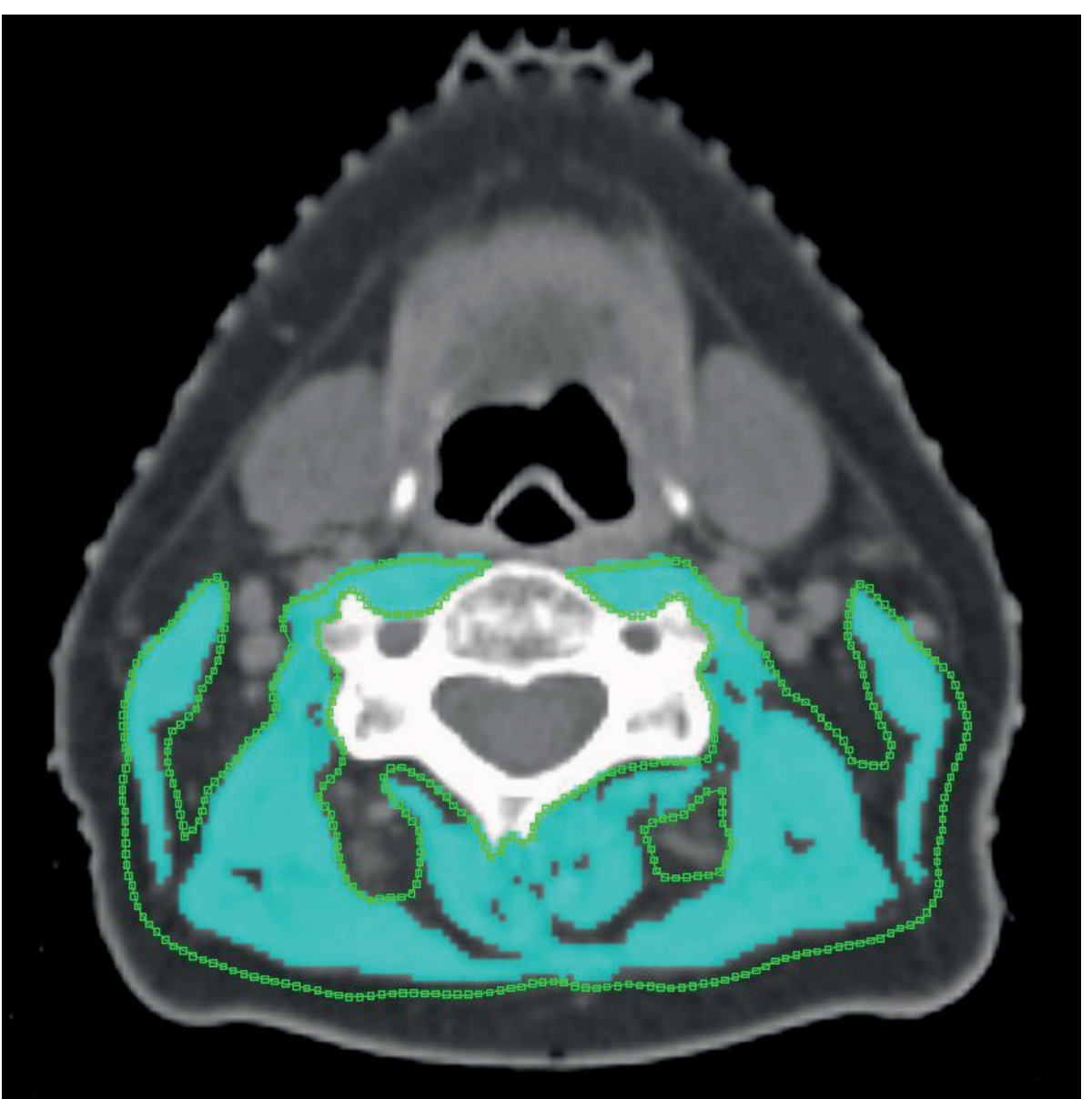

\section{Statistical analysis}

All analyses were performed using SPSS version 25.0 (SPSS Inc. Chicago IL, USA). Continuous data are represented as mean \pm standard deviation (SD). Categorical data are represented as the number and percentage of total. The optimum SMM cut-off value based on CDLT was obtained using the optimal point in a receiver optimum stratification for binary outcomes (in this study, the occurrence of CDLT). The Fisher's exact test, Pearson Chi square test, independent sample t-test and Mann-Whitney $U$ test were used for comparisons between groups where appropriate. The predictive effect of low SMM on CDLT was evaluated using univariate and multivariate logistic regression analysis. Variables with a $p$-value lower than 0.05 in univariate analysis were selected for inclusion in multivariate analysis. Cox proportional hazard regression analysis was used to evaluate the relationship between low SMM and overall survival (OS). Kaplan Meier curves were used to visualize overall survival.

\section{Results}

Of all 279 patients predefined as having an indication for high dose cisplatin 39 patients did not receive any cisplatin and 73 patients were treated with daily cisplatin as part of a clinical study, and were thus excluded. Six patients were treated with induction TPF (docetaxel, cisplatin, fluorouracil), and 4 with weekly cisplatin, and were also excluded. In 4 patients, imaging quality was deemed insufficient. In total, 153 patients who were treated with three-weekly high dose cisplatin were included for analysis. For the overall survival analysis, 41 patients with HPV-positive oropharyngeal cancer were excluded and 112 patients with HPV-negative or unknown status were included.

\section{Patient characteristics}

Patient, disease and outcome characteristics are presented in Table 1. All patients received at least 1 cycle of high dose cisplatin. Patients were predominantly male current smokers and presented with AJCC stage III or IV disease. Of note, almost $50 \%$ of all patients with oropharyngeal cancer had HPV-related oropharyngeal cancer. Approximately half of all patients completed 3 cycles of high dose cisplatin (52.9\%). Two cycles of cisplatin were completed in $22.9 \%$ of patients. In $24.2 \%$ of patients, only 1 cycle of chemotherapy could be completed. CDLT occurred in $24.2 \%$ of patients. The most frequent reason for chemotherapy treatment termination was grade 3 toxicity, being a significant decrease in renal function in $52 \%$, severe nausea in $9 \%$ and infectious disease such as sepsis in $9 \%$ of patients. There were no significant differences in patients characteristics between patient with and without CDLT, apart from a mild renal function impairment prior to start of treatment with an eGFR between 60 and $70(p=0.02)$. 
Table 1. Patient, disease and outcome characteristics.

\begin{tabular}{|c|c|c|c|c|}
\hline Characteristic & $\begin{array}{c}\text { Total } \\
\mathrm{n}=153(\%)\end{array}$ & $\begin{array}{l}\text { With CDLT } \\
\mathrm{n}=37(\%)\end{array}$ & $\begin{array}{c}\text { Without CDLT } \\
\mathrm{n}=116(\%)\end{array}$ & Pvalue \\
\hline \multicolumn{5}{|l|}{ Gender } \\
\hline Men & $112(73.2)$ & $28(75.7)$ & $84(72.4)$ & \multirow[t]{2}{*}{$0.70^{*}$} \\
\hline Women & $41(26.8)$ & $9(24.3)$ & $32(27.6)$ & \\
\hline \multicolumn{5}{|l|}{ Age at diagnosis (years) } \\
\hline Mean (SD) & $59.9(6.7)$ & $61.1(5.9)$ & $59.5(7.0)$ & $0.20^{5}$ \\
\hline \multicolumn{5}{|l|}{ Smoking } \\
\hline $\begin{array}{l}\text { Never } \\
\text { Former }\end{array}$ & $\begin{array}{l}25(16.3) \\
16(10.5)\end{array}$ & $\begin{array}{l}5(13.5) \\
6(16.2)\end{array}$ & $\begin{array}{c}20(17.2) \\
10(8.6)\end{array}$ & \multirow[t]{2}{*}{$0.40^{5}$} \\
\hline Active & $112(73.2)$ & $26(70.3)$ & $86(74.1)$ & \\
\hline \multicolumn{5}{|l|}{ Body mass index } \\
\hline Mean (SD) & $23.7(4.1)$ & $23.6(3.8)$ & $23.8(4.2)$ & $0.82^{5}$ \\
\hline \multicolumn{5}{|l|}{ ACE-27 } \\
\hline 0 & $115(75.2)$ & $28(75.7)$ & $87(75.0)$ & \multirow{3}{*}{$0.20^{\mathrm{s}}$} \\
\hline 1 & $37(24.2)$ & $8(21.6)$ & $29(25.0)$ & \\
\hline 2 & $1(0.7)$ & $1(2.7)$ & $0(0)$ & \\
\hline \multicolumn{5}{|l|}{ Renal function } \\
\hline eGFR $>70$ & $130(85.0)$ & $27(79.4)$ & $103(93.6)$ & \multirow[t]{2}{*}{$0.01^{*}$} \\
\hline eGFR 60-70 & $14(9.2)$ & $7(20.6)$ & $7(6.4)$ & \\
\hline \multicolumn{5}{|l|}{ Tumor site } \\
\hline Oropharynx,HPV+ & $41(26.8)$ & $9(24.3)$ & $32(27.6)$ & \multirow[t]{4}{*}{$0.40^{\sharp}$} \\
\hline Oropharynx, HPV- or unknown & $51(33.3)$ & $12(32.4)$ & $39(33.6)$ & \\
\hline Hypopharynx & $50(32.7)$ & $11(29.7)$ & $39(33.6)$ & \\
\hline Larynx & $11(7.2)$ & $5(13.5)$ & $6(5.2)$ & \\
\hline \multicolumn{5}{|l|}{ T stage } \\
\hline 1 & $15(9.8)$ & $4(10.8)$ & $11(9.5)$ & \multirow[t]{4}{*}{$0.23^{\sharp}$} \\
\hline 2 & $46(30.1)$ & $10(27.0)$ & $36(31.0)$ & \\
\hline 3 & $48(31.4)$ & $12(32.4)$ & $36(31.0)$ & \\
\hline 4 & $44(28.8)$ & $11(29.7)$ & $33(28.4)$ & \\
\hline \multicolumn{5}{|l|}{ N stage } \\
\hline 0 & 19 (12.4) & $3(8.1)$ & $16(13.8)$ & \multirow[t]{6}{*}{$0.53^{\sharp}$} \\
\hline 1 & $17(11.1)$ & $5(13.5)$ & $12(10.3)$ & \\
\hline $2 a$ & $9(5.9)$ & $4(10.8)$ & $5(4.3)$ & \\
\hline $2 b$ & $66(43.1)$ & $13(35.1)$ & $53(45.7)$ & \\
\hline $2 c$ & $35(22.9)$ & $10(27.0)$ & $25(21.6)$ & \\
\hline 3 & $7(4.6)$ & $2(5.4)$ & $5(4.3)$ & \\
\hline
\end{tabular}

\begin{tabular}{lcccc}
\hline AJCC stage & & & & \\
\hline II & $4(2.6)$ & $0(0)$ & $4(3.4)$ & $0.34^{*}$ \\
III & $66(43.1)$ & $14(37.8)$ & $52(44.8)$ & \\
IV & $83(54.2)$ & $23(62.2)$ & $60(51.7)$ & \\
\hline Extracapsular extension & & & & \\
\hline No & $109(71.2)$ & $29(78.4)$ & $80(69.0)$ & $0.27^{*}$ \\
Yes & $44(28.8)$ & $8(21.6)$ & $36(31.0)$ & \\
\hline Number of cisplatin cycles & & & & \\
\hline 1 & $37(24.2)$ & $37(100)$ & - & $n / a$ \\
2 & $35(22.9)$ & - & $35(30.2)$ & \\
3 & $81(52.9)$ & - & $81(69.8)$ & \\
\hline CDLT & & & & \\
\hline Absent & $116(75.8)$ & - & $116(100)$ & \\
Present & $37(24.2)$ & $37(100)$ & & \\
Survival status & & & & \\
Alive & $99(64.7)$ & $21(56.8)$ & $78(67.2)$ & $0.25^{*}$ \\
Deceased & $54(35.3)$ & $16(43.2)$ & $38(32.8)$ & \\
\hline
\end{tabular}

* Fisher's exact test; \# Pearson Chi quare test; $\$$ Independent student's $T$ test

\section{Low SMM as a predictor for CDLT}

A sex-specific cut-off point for low SMM as a predictor for CDLT was formulated using a ROC curve. The AUC of the ROC curve was 0.72 for women (Mann-Whitney $U$ test: $p=0.05$ ) and 0.58 for men (Mann-Whitney $U$ test: $p=0.11$ ). The optimal cut-off value for low SMM was $10.7 \mathrm{~cm}^{2}$ for women and $13.1 \mathrm{~cm}^{2}$ for men. Using this cut-off, $54.9 \%$ of patients had low SMM.

\section{Univariate and multivariate analysis for CDLT}

Table 2 shows patient and disease characteristics of patients with low SMM and normal SMM. Patients with low SMM had a significantly lower BMI $(p<0.01)$ and a higher T stage $(p=0.05)$, and showed a trend towards a higher $\mathrm{N}$ stage $(p=$ 0.09). There were no significant differences in terms of gender or age of patients with and without low SMM. Patients with low SMM experienced CDLT significantly more often than patients with normal SMM $(35.7 \%$ versus $10.1 \% ; p<0.01)$. 
Table 2. Patient characteristics in patients with low and normal SMM

\begin{tabular}{|c|c|c|c|}
\hline All patients & $\begin{array}{l}\text { Patients with low } \\
\text { SMM }\end{array}$ & $\begin{array}{l}\text { Patients with } \\
\text { normal SMMM }\end{array}$ & Pvalue \\
\hline Characteristic & $n=84(\%)$ & $n=69(\%)$ & \\
\hline \multicolumn{4}{|l|}{$\overline{\text { Gender }}$} \\
\hline Men & $64(76.2)$ & $48(69.6)$ & \multirow[t]{2}{*}{$0.37^{*}$} \\
\hline Women & $20(23.8)$ & $21(30.4)$ & \\
\hline \multicolumn{4}{|l|}{ Age at diagnosis (years) } \\
\hline Mean (SD) & $59.9(6.3)$ & $59.8(7.3)$ & $0.95^{5}$ \\
\hline \multicolumn{4}{|l|}{ Smoking } \\
\hline $\begin{array}{l}\text { Never } \\
\text { Former }\end{array}$ & $\begin{array}{l}14(16.7) \\
8(9.5)\end{array}$ & $\begin{array}{l}11(15.9) \\
8(11.6)\end{array}$ & \multirow[t]{2}{*}{$0.92^{\#}$} \\
\hline Active & $62(73.8)$ & $50(72.5)$ & \\
\hline \multicolumn{4}{|l|}{ Body mass index } \\
\hline Mean (SD) & $22.1(3.6)$ & $25.6(3.9)$ & $<0.01^{s}$ \\
\hline \multicolumn{4}{|l|}{ ACE-27 } \\
\hline 0 & $62(73.8)$ & $53(76.8)$ & \multirow{3}{*}{$0.60^{\#}$} \\
\hline 1 & $21(25.0)$ & $16(23.2)$ & \\
\hline 2 & $1(1.2)$ & $0(0)$ & \\
\hline \multicolumn{4}{|l|}{ Renal function } \\
\hline eGFR $>70$ & $73(92.4)$ & $57(87.7)$ & \multirow[t]{2}{*}{$0.40^{*}$} \\
\hline eGFR 60-70 & $6(7.6)$ & $8(12.3)$ & \\
\hline \multicolumn{4}{|l|}{ Tumor site } \\
\hline Oropharynx, HPV+ & $16(19.0)$ & $25(36.2)$ & \multirow[t]{4}{*}{$0.12^{\sharp}$} \\
\hline Oropharynx, HPV- or unknown & $31(36.9)$ & $20(29.0)$ & \\
\hline Hypopharynx & $30(35.7)$ & $20(29.0)$ & \\
\hline Larynx & $7(8.3)$ & $4(5.8)$ & \\
\hline \multicolumn{4}{|l|}{ T stage } \\
\hline 1 & $10(11.9)$ & $5(7.2)$ & \multirow[t]{4}{*}{$0.05^{\#}$} \\
\hline 2 & $18(21.4)$ & $28(40.6)$ & \\
\hline 3 & $27(32.1)$ & $21(30.4)$ & \\
\hline 4 & $29(34.5)$ & $15(21.7)$ & \\
\hline \multicolumn{4}{|l|}{ N stage } \\
\hline 0 & $10(11.9)$ & $9(13.0)$ & \multirow[t]{6}{*}{$0.09^{\sharp}$} \\
\hline 1 & $11(13.1)$ & $6(8.7)$ & \\
\hline $2 a$ & $4(4.8)$ & $5(7.2)$ & \\
\hline $2 b$ & $29(34.5)$ & $37(53.6)$ & \\
\hline $2 c$ & $24(28.6)$ & $11(15.9)$ & \\
\hline 3 & $6(7.1)$ & $1(1.4)$ & \\
\hline
\end{tabular}

\begin{tabular}{lccc}
\hline AJCC stage & & & \\
\hline II & $2(2.4)$ & $2(2.9)$ & $0.11^{\sharp}$ \\
III & $30(35.7)$ & $36(52.2)$ & \\
IV & $52(61.9)$ & $31(44.9)$ & \\
CDLT & & & \\
No & $54(64.3)$ & $62(89.9)$ & $<0.01^{*}$ \\
Yes & $30(35.7)$ & $7(10.1)$ & \\
\hline
\end{tabular}

Bold indicates a significant difference between groups with $p<0.05$. Cursive indicates a $p$ value $<$ 0.10 .

* Fisher's exact test; \# Pearson Chi square test; \$ Independent student's T test

In Table 3, the univariate and multivariate analysis for the occurrence of CDLT are shown. In univariate analysis, only low SMM (OR 3.75 [95\% CI 1.58-8.90], p $<0.01$ ) and a mild renal function impairment with an eGFR of $60-70$ (OR 3.82 [95\% Cl 1.23 - 11.81], $\mathrm{p}=0.02$ ) were associated with the occurrence of CDLT. In multivariate analysis, both low SMM (OR 3.99 [95\% Cl $1.56-10.23], \mathrm{p}=0.01)$ and a mild renal function impairment (OR 5.40 [95\% Cl $1.57-18.65]$, $\mathrm{p}<0.01$ ) remained associated with the occurrence of CDLT.

\section{Survival analysis}

Table 4 shows univariate and multivariate Cox regression analysis for OS in HPVnegative patients or patients with unknown HPV-status $(n=112)$. In univariate Cox regression analysis, by far the most important prognosticator was HPV-status of the tumor; with patients with HPV-related oropharyngeal cancer having a better prognosis than other patients in this cohort (HR $0.07[95 \% \mathrm{Cl} 0.02-0.31], \mathrm{p}<0.01)$. 
Table 3. Univariate and multivariate logistic regression analysis for prediction of CDLT Univariate analysis Multivariate analysis

Odds Ratio $(95 \% \mathrm{Cl}) \quad$ p-value $\quad$ Odds Ratio $(95 \% \mathrm{Cl}) \quad \mathrm{p}$-value

\begin{tabular}{|c|c|c|c|c|}
\hline & Odds Ratio (95\% Cl) & p-value & Odds Ratio (95\% Cl) & p-value \\
\hline Gender & & & & \\
\hline Male & Ref & & & \\
\hline Female & $0.84(0.36-1.98)$ & 0.70 & & \\
\hline Age at diagnosis & & & & \\
\hline (years) & $1.04(0.98-1.10)$ & 0.20 & & \\
\hline BMI at diagnosis & & & & \\
\hline$\left(\mathrm{kg} / \mathrm{m}^{2}\right)$ & $0.99(0.90-1.08)$ & 0.82 & & \\
\hline Tumor site & & & & \\
\hline Oropharynx HPV+ & Ref & & & \\
\hline Oropharynx HPV-/unknown & $1.09(0.41-2.92)$ & 0.86 & & \\
\hline Hypopharynx & & & & \\
\hline Larynx & $2.96(0.72-12.00)$ & 0.13 & & \\
\hline & $1.00(0.37-2.72)$ & 1.00 & & \\
\hline AJCC stage & & & & \\
\hline$\|-\| I I$ & Ref & & & \\
\hline IV & $1.53(0.72-3.27)$ & 0.27 & & \\
\hline Renal function & & & & \\
\hline eGFR $>70$ & Ref & & Ref & \\
\hline eGFR 60-70 & $3.82(1.23-11.81)$ & 0.02 & & $<0.01$ \\
\hline Low SMM & & & Ref & \\
\hline No & Ref & & $3.99(1.56-10.23)$ & \\
\hline Yes & $3.75(1.58-8.90)$ & $<0.01$ & $5.40(1.57-18.65)$ & 0.01 \\
\hline ACE-27 & & & & \\
\hline 0 & Ref & & & \\
\hline 1 or 2 & $0.96(0.41-2.28)$ & 0.94 & & \\
\hline Smoking & & & & \\
\hline No & Ref & & & \\
\hline Former & $2.40(0.59-9.82)$ & 0.22 & & \\
\hline Active & $1.21(0.41-3.54)$ & 0.73 & & \\
\hline
\end{tabular}

Bold indicates a significant difference between groups.
Table 4. Univariate and multivariate analysis for overall survival

\begin{tabular}{|c|c|c|c|c|}
\hline & \multicolumn{2}{|c|}{ Univariate analysis } & \multicolumn{2}{|c|}{ Multivariate analysis } \\
\hline & Hazard Ratio $(95 \% \mathrm{Cl})$ & $p$-value & Hazard Ratio $(95 \% \mathrm{Cl})$ & p-value \\
\hline \multicolumn{5}{|l|}{ Gender } \\
\hline Male & Ref & & & \\
\hline Female & $1.06(0.57-1.98)$ & 0.86 & & \\
\hline \multicolumn{5}{|l|}{ Age at diagnosis } \\
\hline (years) & $1.02(0.97-1.06)$ & 0.58 & & \\
\hline \multicolumn{5}{|l|}{ BMI at diagnosis } \\
\hline$\left(\mathrm{kg} / \mathrm{m}^{2}\right)$ & $0.93(0.87-0.99)$ & 0.03 & $0.94(0.88-1.00)$ & 0.07 \\
\hline \multicolumn{5}{|l|}{ Tumor site } \\
\hline Oropharynn HPV+ & Excluded* & & & \\
\hline Oropharynx HPV-/unknown & Ref & & & \\
\hline Hypopharynx & & & & \\
\hline \multirow{2}{*}{ Larynx } & $1.86(0.74-4.69)$ & 0.19 & & \\
\hline & $1.46(0.81-2.61)$ & 0.21 & & \\
\hline \multicolumn{5}{|l|}{ AJCC stage } \\
\hline 2 and 3 & Ref & & Ref & \\
\hline 4 & $3.57(1.79-7.14)$ & $<0.01$ & $3.40(1.69-6.81)$ & $<0.01$ \\
\hline \multicolumn{5}{|l|}{ CDLT } \\
\hline № & Ref & & Ref & \\
\hline Yes & $2.11(1.15-3.89)$ & 0.02 & $2.10(1.13-3.90)$ & 0.02 \\
\hline \multicolumn{5}{|l|}{ Low SMM } \\
\hline No & Ref & & & \\
\hline Yes & $1.23(0.71-2.16)$ & 0.46 & & \\
\hline \multicolumn{5}{|l|}{ ECE } \\
\hline № & Ref & & & \\
\hline Yes & $1.10(0.55-2.19)$ & 0.80 & & \\
\hline \multicolumn{5}{|l|}{ ACE-27 } \\
\hline . & Ref & & & \\
\hline 1 or 2 & $0.79(0.41-1.53)$ & 0.48 & & \\
\hline
\end{tabular}

Bold indicates a significant difference between groups.

* HPV-related oropharyngeal cancer: HR 0.07 (95\% Cl $0.02-0.311, \mathrm{p}<0.01$

In univariate Cox regression analysis, low SMM was not a significant prognosticato (HR 1.23 [95\% Cl $0.71-2.16], p=0.46$ ) for OS, as visualized in Figure 2. In contrast, the occurrence of CDLT was significantly associated with a decreased OS (HR 2.11 [95\% Cl 1.15 - 3.89], $p=0.02$ ), as visualized in Figure 3. Other significant prognosticators for OS were AJCC stage IV disease (HR $3.57[95 \% \mathrm{Cl} 1.79-7.14), \mathrm{p}<0.01)$ and BM (HR 0.93 [95\% Cl $0.87-0.99], p=0.03)$, with a higher BMI being associated with significantly better OS. In multivariate regression analysis only AJCC stage IV disease and CDLT remained significantly associated with decreased OS. 
Figure 2: Kaplan Meier survival curve for low SMM in HPV negative patients.

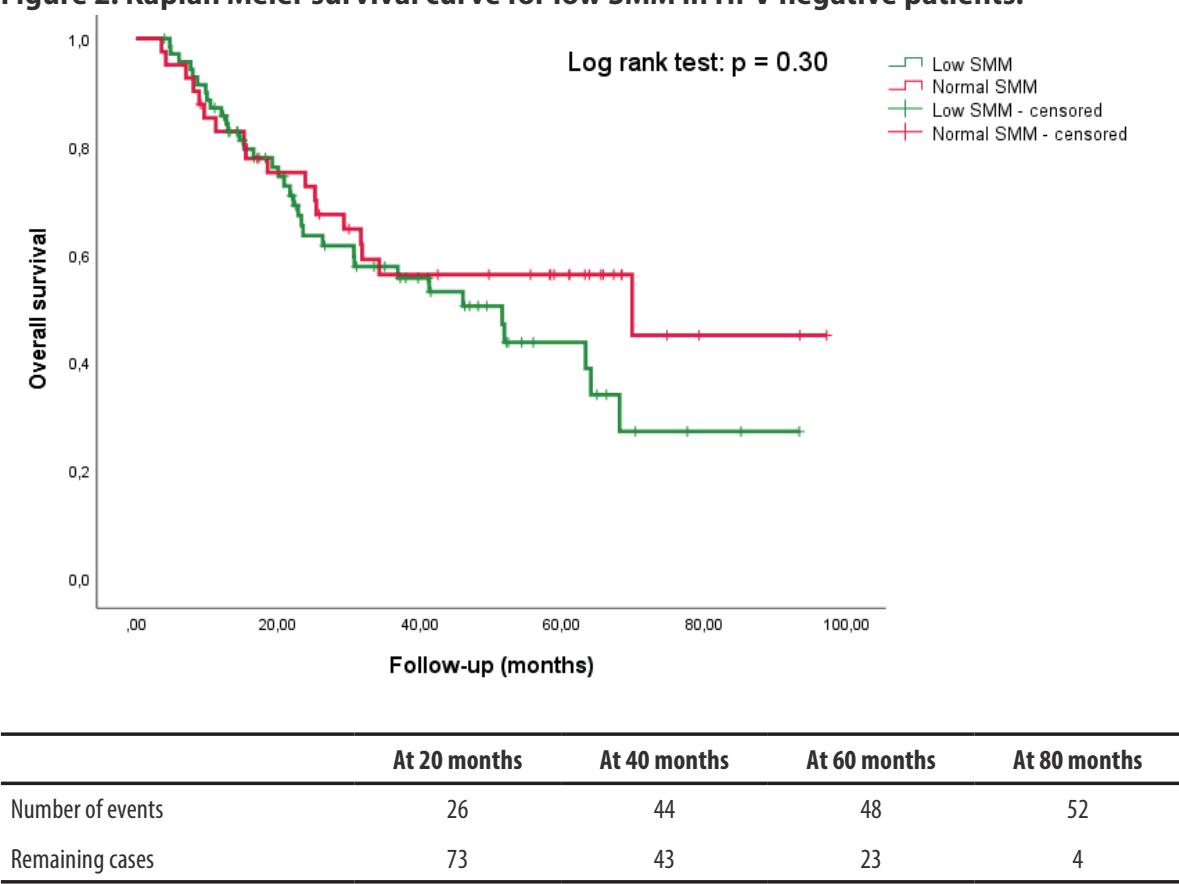

Figure 3: Kaplan Meier survival curve for CDLT in HPV negative patients

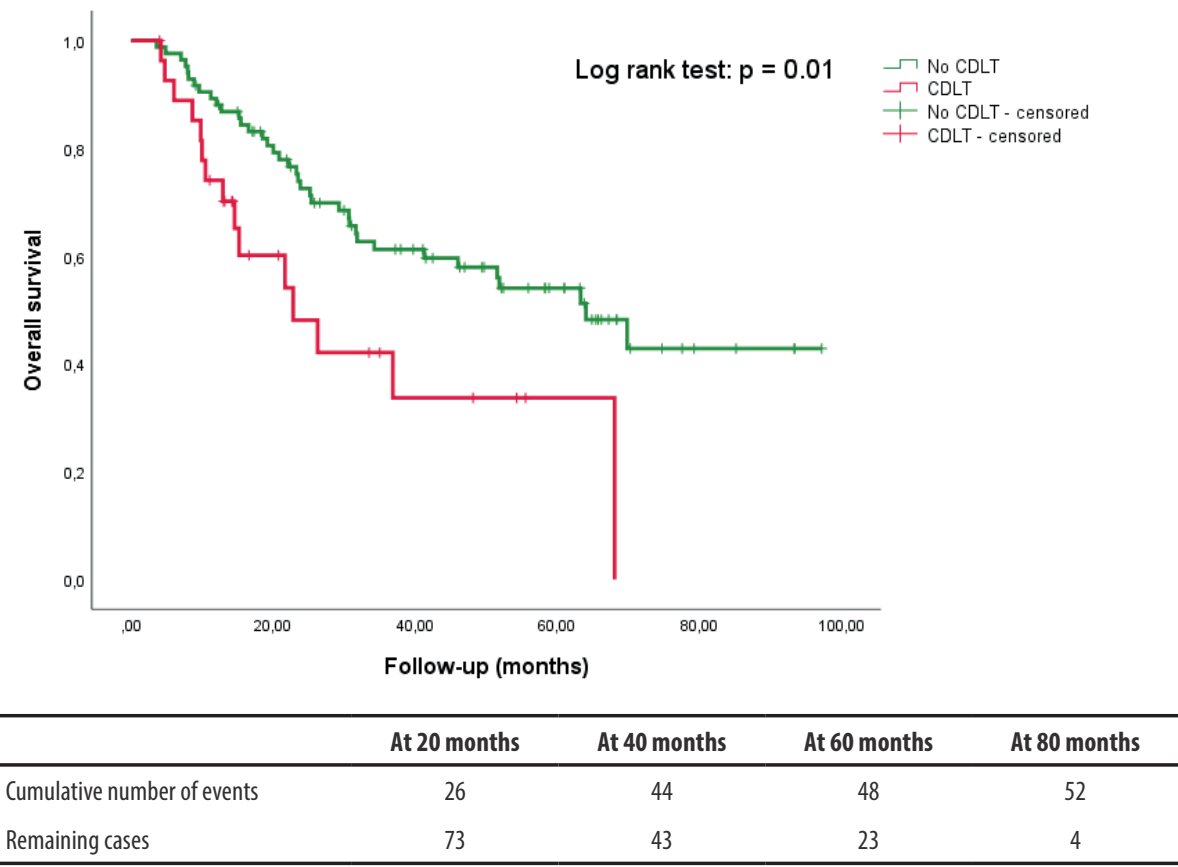

\section{Discussion}

Low SMM is associated with an increase in chemotherapy related toxicity and CDLT in a variety of cancer types. Our study also shows this relationship in HNSCC patients treated with primary CRT with high-dose cisplatin. Patients with low SMM had a trifold risk of experiencing CDLT compared to patients with normal SMM in this study. Although patients with low SMM did not have a decreased OS, patients who experienced CDLT did have a significantly decreased OS. This study adds to the mounting evidence that there is a clear relationship between low SMM and the occurrence of CDLT in HNSCC patients treated with high dose cisplatin. $9,16,21,22$

Platinum-based chemotherapy is routinely used in the curative treatment of LAHNSCC to enhance the antitumor effect of radiation. Several treatment schemes and dosing levels are available for platinum-based chemotherapy in HNSCC. Level 1 evidence is available for the improvement of locoregional control and overall survival with concurrent CRT with three three-weekly cycles of high dose cisplatin at a dose level of $100 \mathrm{mg} / \mathrm{m}^{2}$ BSA. ${ }^{2}$ Despite irrefutable efficacy, the toxicity of treatment with high dose cisplatin is a well-known problem in daily clinical practice. Early chemotherapy termination due to unacceptable toxicity occurs in approximately $30 \%$ of patients, and is associated with a marked decrease in overall survival (52\% versus $72 \%$ in 3 -year survival) as well as increase in longterm morbidity of treatment. In recent years, several large clinical trials have investigated de-escalation strategies with weekly low-dose cisplatin or cetuximab as radiosensitizer in HNSCC, but these trials concluded that concurrent CRT with high-dose cisplatin remains the preferred treatment option with the highest survival benefit. ${ }^{23-25}$

There is an evident clinical need for improved risk assessment in patients planned for high-dose cisplatin treatment. Several risk factors for cisplatin toxicity are already established absolute contra-indications, such as a decreased renal function with an eGFR $<60$, severe hearing loss or poor functional WHO-status. Better knowledge on relative contraindications is needed to identify patients who may benefit from modified treatments. Low SMM is a radiological biomarker that may aid in the identification of those patients at high risk of cisplatin related toxicity that would otherwise not have been identified. ${ }^{26}$

Over the last decade, the body composition of cancer patients has been researched extensively using diagnostic computer tomography (CT) imaging. ${ }^{2}$ Recent retrospective studies in a variety of cancer types have shown an 
association between low SMM, sometimes termed sarcopenia, and the occurrence of chemotherapeutic toxicity and CDLT. ${ }^{10}$ Several hypothesis have been proffered One hypothesis behind this relationship is that most (hydrophilic) chemotherapy, including cisplatin, mainly distributes into the fat-free body mass, of which skeletal muscle mass is the largest contributor. ${ }^{11,28}$ Patients with low SMM and normal or high fat mass may receive a relatively higher dose of chemotherapy than is anticipated using a standard dosing regimen based on BSA. Previous research has shown that drug dosing based on BSA poorly predicts plasma drug concentrations of most cytotoxic drugs in individual patients, including cisplatin. ${ }^{29,30}$ Currently, a prospective study investigating this relationship in HNSCC patients is ongoing.

It may also be that low SMM reflects an overall poorer physical functioning in patients, which is not as distinctly found as using other routinely used risk stratification methods. In recent years, there has been increased interest in the supportive care of cancer patients undergoing chemotherapy, including increased interest in guided exercise and nutritional support during cancer treatment. A randomized controlled trial in breast cancer patients undergoing several physical activity programs showed a positive effect on treatment tolerance and fatigue. ${ }^{31} \mathrm{~A}$ recently published randomized controlled trial in rectal cancer patients undergoing neoadjuvant CRT showed a significant increase in SMM in patients who followed an exercise program during neoadjuvant chemotherapy, compared to patients who did not. ${ }^{32}$ A recent study in breast cancer patients undergoing adjuvant chemotherapy did not show a difference in chemotherapy completion in patients participating in an exercise intervention, but it did show a significant decrease in hospitalization during treatment. ${ }^{33}$ Besides exercise and nutritional support during cancer treatment, 'prehabilitation' with exercise and nutritional support prior to start of treatment are likely to increase treatment tolerance. However, limited time between diagnosis and start of treatment may decrease the ability to effectively implement a prehabilitation program in patients undergoing primary CRT.

Feasibility studies in patients with HNSCC have shown that muscle resistance training programs in patients undergoing chemoradiotherapy or radiotherapy are feasible and show high patient satisfaction. ${ }^{34,35}$ Whether such interventions also provide benefit in terms of overall survival is unknown, but low SMM prior to start of treatment may be an indicator that a patient may benefit from intensified supportive care in terms of physical exercise and nutritional support. Pretreatment low SMM may also be used as an argument for an intended treatment de-escalation choice, such as weekly low-dose cisplatin, to maximize treatment adherence and cumulative cisplatin dose administered.
Several limitations to this study need to be addressed. Due to the retrospective nature of the research, not all relevant research parameters for body composition or nutritional status were measured or documented during normal clinica practice. Because of the academic nature of the tertiary referral center this study was conducted in, a relatively large percentage of patients was excluded because of a trial-based treatment regimen (weekly or daily cisplatin). In the present study CDLT was defined as any toxicity resulting in a cumulative cisplatin dose of less than $200 \mathrm{mg} / \mathrm{m} 2$; it is generally accepted that at least a dose of $200 \mathrm{mg} / \mathrm{m} 2$ should be administered to be sufficiently effective. ${ }^{3,4}$ In the previous study of Wendrich et al, CDLT was defined as any toxicity resulting in any chemotherapy dosereduction of $\geq 50 \%$ (e.g. due to neutropenia or nephrotoxicity), a postponement of treatment of $\geq 4$ days (e.g. in the case of bone marrow suppression) or a definite termination of chemotherapy after the first or second cycle of therapy. Despite slightly different definitions of CDLT, the conclusions of both studies were comparable: a threefold significant higher incidence of CDLT in SMM patients ( $35.7 \%$ vs. $10.1 \%$ and $44.3 \%$ vs. $13.7 \%$ ). In both studies patients experiencing CDLT had a significantly lower overall survival than patients who did not.

In the current study, we decided not to use a previously published multivariate formula to calculate CSMA at the level of L3, but rather use CSMA at the level of C3 directly to assess SMM. This better allowed us to formulate a sex-specific cut-off point for low SMM, as is commonly done in other areas of oncological research rather than use a single cut-off point. It is known that women have less SMM than men. ${ }^{36}$. Sex is part of the previously published prediction formula for translation of CSMA at level of C3 to CSMA at level of L3; as such sex is implicitly already accounted for using this method. This choice does hinder direct comparison to our previous results. It should be noted that the incidence of low SMM as well as the trifold risk of CDLT in patients with low SMM is equal in both our previous ${ }^{9}$ and this current study, and compares to results in other studies.

\section{Conclusion}

This study validates the previous findings that pre-treatment low SMM is significantly associated with CDLT in LA-HNSCC patients treated with primary CRT with high dose cisplatin. Pre-treatment low SMM alone was not a prognostic factor for OS, but CDLT was. Routine SMM assessment may allow for CDLT risk assessment and identification of those patients who may benefit from treatment modifications and from interventions to increase SMM. 


\section{References}

1. Grégoire V, Lefebvre J-L, Licitra L, Felip E. Squamous cell carcinoma of the head and neck: EHNS-ESMO-ESTRO Clinical Practice Guidelines for diagnosis, treatment and follow-up. Ann Oncol. 2010;21:v184-v186.

2. Szturz P, Wouters K, Kiyota N, et al. Low-Dose vs. High-Dose Cisplatin: Lessons Learned From 59 Chemoradiotherapy Trials in Head and Neck Cancer. Front Oncol. 2019;9.

3. Pignon J-P, Maître A le, Maillard E, Bourhis J. Meta-analysis of chemotherapy in head and neck cancer (MACH-NC): An update on 93 randomised trials and 17,346 patients. Radiother Oncol. 2009;92(1):4-14.

4. Strojan P, Vermorken JB, Beitler JJ, et al. Cumulative cisplatin dose in concurrent chemoradiotherapy for head and neck cancer: A systematic review. Eisele DW, ed. Head Neck. 2016;38(S1):E2151-E2158.

5. Spreafico A, Huang $\mathrm{SH}, \mathrm{Xu} \mathrm{W}$, et al. Impact of cisplatin dose intensity on human papillomavirusrelated and -unrelated locally advanced head and neck squamous cell carcinoma. Eur J Cancer. 2016;67:174-182.

6. Al-Mamgani A, de Ridder M, Navran A, Klop WM, de Boer JP, Tesselaar ME. The impact of cumulative dose of cisplatin on outcome of patients with head and neck squamous cell carcinoma. Eur Arch Oto-Rhino-Laryngology. 2017;274(10):3757-3765.

7. Ang K, Zhang Q, Wheeler RH, et al. A phase III trial (RTOG 0129) of two radiation-cisplatin regimens for head and neck carcinomas (HNC): Impact of radiation and cisplatin intensity on outcome. J Clin Oncol. 2010;28(15_suppl):5507-5507.

8. Forastiere AA, Zhang Q, Weber RS, et al. Long-Term Results of RTOG 91-11: A Comparison of Three Nonsurgical Treatment Strategies to Preserve the Larynx in Patients With Locally Advanced Larynx Cancer. J Clin Oncol. 2013;31(7):845-852.

9. Wendrich AW, Swartz JE, Bril Sl, et al. Low skeletal muscle mass is a predictive factor for chemotherapy dose-limiting toxicity in patients with locally advanced head and neck cancer. Oral Oncol. 2017;71:26-33.

10. Rier HN, Jager A, Sleijfer S, Maier AB, Levin M. The Prevalence and Prognostic Value of Low Muscle Mass in Cancer Patients: A Review of the Literature. Oncologist. 2016;21 (11):1396-1409.

1. Hopkins JJ, Sawyer MB. Interactions of lean soft-tissue and chemotherapy toxicities in patients receiving anti-cancer treatments. Cancer Chemother Pharmacol. 2018;82(1):1-29.

12. Cruz-Jentoft AJ, Baeyens JP, Bauer JM, et al. Sarcopenia: European consensus on definition and A Barcopenia in Older People. Age Ageing. 2010;39(4):412-423.

13. Bril SI, Pezier TF, Tijink BM, Janssen LM, Braunius WW, Bree R. Preoperative low skeletal muscle mass as a risk factor for phar mass as a

14. Stone L, Olson B, Mowery A, et al. Association Between Sarcopenia and Mortality in Patients Undergoing Surgical Excision of Head and Neck Cancer. JAMA Otolaryngol Neck Surg. 2019;145(7):647.

15. Zwart AT, Hoorn A, Ooijen PMA, Steenbakkers RJHM, Bock GH, Halmos GB. CT-measured skeletal muscle mass used to assess frailty in patients with head and neck cancer. J Cachexia Sarcopenia Muscle. 2019;10(5):1060-1069.

16. Sealy MJ, Dechaphunkul T, van der Schans CP, et al. Low muscle mass is associated with early termination of chemotherapy related to toxicity in patients with head and neck cancer. Clin Nutr. 2020;39(2):501-509.

17. Paleri V, Wight RG, Silver CE, et al. Comorbidity in head and neck cancer: a critical appraisal and recommendations for practice. Oral Oncol. 2010;46(10):712-719.

18. Swartz JE, Pothen AJ, Wegner I, et al. Feasibility of using head and neck CT imaging to assess skeletal muscle mass in head and neck cancer patients. Oral Oncol. 2016;62:28-33.

19. B. Heymsfield S, Wang Z, Baumgartner RN, Ross R. Human Body Composition: Advances in Models and Methods. Annu Rev Nutr. 1997;17(1):527-558.
20. Prado $\mathrm{CM}$, Lieffers JR, McCargar LJ, et al. Prevalence and clinical implications of sarcopenic obesity in patients with solid tumours of the respiratory and gastrointestinal tracts: obesity in patients with solid tumours of the respirator

21. Shodo R, Yamazaki K, Ueki Y Takahashi T, Horii A Sarcopenia predicts a poor treatment outcome in patients with head and neck squamous cell carcinoma receiving concurrent chemoradiotherapy. Eur Arch Oto-Rhino-Laryngology. Published online August 8, 2020.

22. Ganju RG, Morse R, Hoover A, TenNapel M, Lominska CE. The impact of sarcopenia on tolerance of radiation and outcome in patients with head and neck cancer receiving chemoradiation. Radiother Oncol. 2019;137:117-124.

23. Mehanna H, Robinson M, Hartley A, et al. Radiotherapy plus cisplatin or cetuximab in lowrisk human papillomavirus-positive oropharyngeal cancer (De-ESCALaTE HPV): an open-labe randomised controlled phase 3 trial. Lancet. 2019:393(10166):51-60.

24. Gillison ML, Trotti AM, Harris J, et al. Radiotherapy plus cetuximab or cisplatin in human papillomavirus-positive oropharyngeal cancer (NRG Oncology RTOG 1016): a randomised multicentre, non-inferiority trial. Lancet. 2019;393(10166):40-50.

25. Gebre-Medhin M, Brun E, Engström P, et al. ARTSCAN III: A Randomized Phase III Study Comparin Chemoradiotherapy With Cisplatin Versus Cetuximab in Patients With Locoregionally Advanced Head and Neck Squarmous Cell Cancersus Cetuximab in Patients With Locoregto

26. Economopoulou P de Bree R, Kotsantis I, Psyrri A. Diagnostic Tumor Markers in Head and Neck Squmer

27. Prado CM Cushen SJ, Orsso CE Ryan AM Sarcopenia and cachexia in the era of obesity: clinica Prado CM, Cushen SJ, Orsso CE, Ryan AM. Sarcopenia and cach
and nutritional impact. Proc Nutr Soc. 2016;75(2):188-198.

28. Urien $S$, Lokiec $F$. Population pharmacokinetics of total and unbound plasma cisplatin in adult patients. Br J Clin Pharmacol. 2004;57(6):756-763.

29. Felici A, Verweij J, Sparreboom A. Dosing strategies for anticancer drugs: the good, the bad and body-surface area. Eur J Cancer. 2002;38(13):1677-1684. doi:10.1016/S0959-8049(02)00151-X

30. Mathijssen RHJ, de Jong FA, Loos WJ, van der Bol JM, Verweij J, Sparreboom A. Flat-Fixed Dosing Versus Body Surface Area-Based Dosing of Anticancer Drugs in Adults: Does It Make Difference? Oncologist. 2007;12(8):913-923.

31. van Waart $\mathrm{H}$, Stuiver MM, van Harten WH, et al. Effect of Low-Intensity Physical Activity and Moderate- to High-Intensity Physical Exercise Durng Adjuvant Chemotherapy on Physica Fitness, Fatigue, and Chemotherapy Completion Rates: Results of the PACES Randomized Clinical Trial. J Clin Oncol. 2015;33(17):1918-1927.

32. Moug SJ, Barry SJE, Maguire $\mathrm{S}$, et al. Does prehabilitation modify muscle mass in patients with rectal cancer undergoing neoadjuvant therapy? A subanalysis from the REx randomised controlled trial. Tech Coloproctol. 2020;24(9):959-964.

33. Mijwel S, Bolam KA, Gerrevall J, Foukakis T, Wengström Y, Rundqvist H. Effects of Exercise on Chemotherapy Completion and Hospitalization Rates: The OptiTrain Breast Cancer Trial. Oncologist. 2020;25(1):23-32.

34. Sandmael JA, Bye A, Solheim TS, et al. Feasibility and preliminary effects of resistance training and nutritional supplements during versus after radiotherapy in patients with head and neck cancer: A pilot randomized trial. Cancer. 2017;123(22):4440-4448.

35. Brown TE, Banks MD, Hughes BGM, Lin CY, Kenny LM, Bauer JD. Randomised controlled trial of early prophylactic feeding vs standard care in patients with head and neck cancer $\mathrm{Br} J \mathrm{Cancer}$ 2017;117(1):15-24

36. Janssen I, Heymsfield SB, Wang Z, Ross R. Skeletal muscle mass and distribution in 468 men and women aged 18-88 yr. J Appl Physiol. 2000:89(1):81-88. 


\section{Chapter 6}

Preoperative low skeletal muscle mass as a risk factor for pharyngocutaneous fistula and decreased overall survival in patients undergoing laryngectomy

Sandra I. Bril, Thomas F. Pezier, Bernard M. Tijink, Luuk M. Janssen, Weibel W. Braunius, Remco de Bree

Head Neck. 2019 Jun;41(6):1745-1755 


\section{Abstract}

Background Low skeletal muscle mass (SMM) is associated with postoperative complications, prolonged hospital stay, and short overall survival (OS) in surgical oncology. We aimed to investigate this association in patients undergoing total laryngectomy (TL).

Methods A retrospective study was performed of patients undergoing TL. SMM was measured using CT or MRI scans at the level of the third cervical vertebra (C3).

Results In all, 235 patients were included. Low SMM was observed in 109 patients (46.4\%). Patients with low SMM had more pharyngocutaneous fistulas (PCFs) than patients with normal SMM (34.9\% vs $20.6 \% ; \mathrm{P}=002$ ) and prolonged hospital stay (median, 17 vs 14 days; $\mathrm{P}<0.001$ ). In multivariate analysis, low SMM (hazard ratio, 1.849; $95 \%$ confidence interval, 1.202-2.843) and high N stage were significant prognosticators of decreased OS.

Conclusion Low SMM is associated with PCF and prolonged hospital stay in patients undergoing TL. Low SMM is an independent prognostic factor for shorter OS.

\section{Introduction}

Total laryngectomy with or without (partial) pharyngectomy (TL), often followed by postoperative (chemo)radiotherapy is a curative treatment option for patients with advanced stage primary laryngeal or hypopharyngeal cancer. Total laryngectomy can also be used to salvage patients with recurrent disease after failure of initial organ preserving treatment with (chemo)radiotherapy or in patients without (current) cancer but with a dysfunctional larynx., ${ }^{1,2}$ Total laryngectomy is an invasive surgical procedure and is associated with significant morbidity and mortality, as well as a reduced quality of life after surgery. ${ }^{3}$

Postoperative complications including the occurrence of a pharyngocutaneous fistula (PCF) are common and difficult to treat problems after TL. ${ }^{1-4}$ Up to thirty percent of patients develop PCF, which may require additional surgery, prolong feeding tube dependency and increases hospital stay. ${ }^{5}$ Previously described risk factors for the occurrence of a PCF include prior chemoradiotherapy with platinum-based chemotherapy, hypopharyngeal cancer, extensive pharyngeal resection and reconstruction, additional neck dissection and low BMI. The occurrence of a PCF may also cause delay of postoperative (chemo)radiotherapy, thus jeopardizing optimal oncological treatment. 6,7

The radiological assessment of body composition has increasingly gained attention in oncological research over the last decade..$^{8,9}$ Specifically a low skeletal muscle mass (SMM), also termed sarcopenia, has been related to negative outcomes in variety of tumour types and treatments. In geriatric patients, sarcopenia is defined as a geriatric syndrome characterized by the age-related loss of muscle mass and/ or muscle function or decreased physical status. ${ }^{10}$ In oncological patients, often only SMM is assessed as muscle function is rarely measured during routine clinical practice. ${ }^{11}$

In oncological patients, skeletal muscle mass is most commonly assessed on abdominal computed tomography (CT) imaging at the level of the third lumbar vertebra (L3). ${ }^{12,13}$ Abdominal CT is routinely performed during diagnostic work-up and follow-up of many cancer patients, and thus imaging is routinely available for analysis without any extra burden for the patient or healthcare-related costs.

Low SMM has been related to more postoperative complications, prolonged hospital stay and decreased survival in surgical oncology. ${ }^{14,15}$ In head and neck cancer, the predictive and prognostic value of low SMM has not yet been 
researched as thoroughly. Abdominal CT imaging is not routinely performed in head and neck cancer patients, and is often only available in a preselected patient group with advanced disease and high risk features for distant metastasis. Recently, a novel SMM assessment method at the level of the third cervical vertebra (C3) was published. ${ }^{16}$ Imaging at the level of C 3 is almost always available in head and neck cancer patients, allowing for the routine assessment of SMM.

In this paper, we aim to investigate whether preoperative low SMM, as measured using $\mathrm{CT}$ or $\mathrm{MRI}$ imaging at the level of $\mathrm{C} 3$, is a significant predictor of postoperative complications including PCF, prolonged hospital stay and decreased overal survival in a large consecutive cohort of patients undergoing TL for any indication.

\section{Methods}

The design of this study was approved by the Medical Ethical Research Committee of the University Medical Center Utrecht (ID 17-365/C). The research was conducted in accordance with the Declaration of Helsinki.

\section{Patient and study design}

A retrospective case note review was performed of all consecutive patients who had undergone TL between January 2008 and May 2017 at the University Medical Center, Utrecht, The Netherlands, a tertiary referral center for head and neck cancer patients. All patients were discussed in the local multidisciplinary tumour board prior to and after surgery. Patients without recent CT or MRI scans (less than 3 months) of the head and neck area prior to TL were excluded. Patients who had severe dental artifacts at the level of $\mathrm{C} 3$ that impeded accurate assessment of SMM were also excluded.

Patients' demographic, staging, treatment and outcome data were collected using electronic patient records. Both versions 6 and 7 of the AJCC manual were used for staging as the study period straddled the change in 2009. ${ }^{17}$ All patients were discussed in local tumour board meetings and underwent TL with or without (partial) pharyngectomy and with or without additional lymph node dissection either as a primary treatment, a salvage treatment or a functional treatment for a dysfunctional larynx.

Five dedicated head and neck surgical oncologists performed all tota laryngectomies during the time period. Operating records were checked for details of the surgery, neck dissection and primary pharyngeal closure or flap reconstruction of the pharynx. Prior treatment with radiotherapy or chemoradiotherapy for head and neck cancer was recorded. Postoperative adjuvant treatment was also recorded. The American Society of Anesthesiologist's physical status classification was recorded as a surrogate marker for comorbidities. ${ }^{18}$ Postoperative complications were graded according to the Clavien-Dindo classification of Surgica Complications. ${ }^{19}$ Severe complications were defined as Clavien-Dindo grade 3A or higher. Of specific interest was the occurrence of PCF which was scored separately to the other postoperative complications. The occurrence of PCF was defined as a clinical fistula requiring any form of conservative or surgical treatment. Duration of hospital stay was recorded as the time in days between the date of $T L$ and date of first hospital discharge.

Follow-up/survival data was retrieved up until the $31^{\text {st }}$ of August, 2017. Overal survival (OS) was defined as the time elapsed between the date of total laryngectomy and the date of death. Disease-specific survival (DSS) was defined as any patient who had died as a result of the current head and neck cancer diagnosis or as a result of the surgical procedure. Survival status was checked in the patient medical records in our hospital. Patients are routinely contacted during the first five years of follow-up after TL.

\section{Skeletal muscle mass measurement}

Skeletal muscle mass was measured on pre-treatment CT or MRI scans of the head and neck area at the level of C3 using a previously published method. Whenever possible, CT imaging was used instead of MRI. In brief, the first slide at the level of $\mathrm{C} 3$ when scrolling from caudal to cranial direction to show both transverse processes and the entire vertebral arc was selected for segmentation of skeletal muscle (SM) tissue. For CT imaging, SM area was defined as the pixe area within a radiodensity between -29 and +150 Hounsfield Units (HU), which is specific for SM tissue. ${ }^{20}$ For MRI, SM tissue was carefully segmented and any intramuscular fatty tissue was manually excluded. Segmentation of SM tissue was manually performed by a single researcher (S.B.) using the commercially available software package SliceOmatic (Tomovision, Canada). An example of SM tissue segmentation at the level of $\mathrm{C} 3$ is shown in Figure 1. After a learning period, the measurement of SMM requires 5 to 10 minutes per CT scan, and up to 15 minutes per MRI scan. 
Figure 1. Example of segmentation of skeletal muscle tissue at the level of $\mathrm{C3}$.

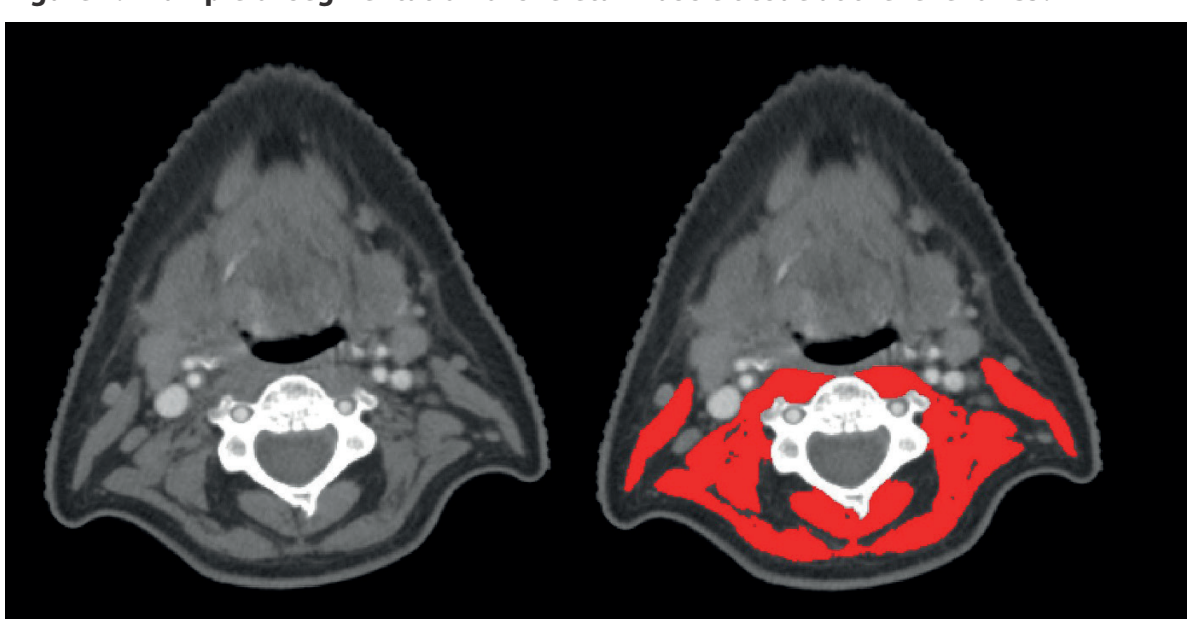

Two identical axial CT-slides at the level of the third cervical vertebra (C3); the left CT slide shows the skeletal muscle tissue unsegmented, while the right image shows the paravertebral muscles and both sternocleidomastoid muscles segmented in red, using SliceOmatic software. Hounsfield unit (HU) ranges are set at -29 to $+150 \mathrm{HU}$ for optimal identification of skeletal muscle tissue.

From SM area at C3, SM area at the level of L3 was predicted using the previously published Formula $1 .{ }^{16}$ The SM area at L3 was then normalized for height to calculate the lumbar skeletal muscle index (lumbar SMI), as shown in Formula $2 .{ }^{12}$ Low SMM was defined as a lumbar SMI lower than $43.2 \mathrm{~cm}^{2} / \mathrm{m}^{2}$. This recently published cutoff value was established in a separate cohort of head and neck cancer patients. $^{2}$

\section{Formula 1:}

CSMA at L3 $\left(\mathrm{cm}^{2}\right)=27.304+1.363 *$ CSMA at C3 $\left(\mathrm{cm}^{2}\right)+0.640 *$ Weight $(\mathrm{kg})+$ $26.442 *$ Gender (Gender $=1$ for female, 2 for male) $-0.671 *$ Age (years)

\section{Formula 2:}

Lumbar SMI $\left(\mathrm{cm}^{2} / \mathrm{m}^{2}\right)=$ CMSA at L3 $/\left(\right.$ height $\left.^{2}\right)$

\section{Statistical analysis}

Categorical data are represented as a number and percentage of the total. All statistical analyses were performed using the IBM SPSS Statistics version 21.0 software package (Chicago, Illinois, USA). A test for normality (KolmogorovSmirnoff test) and histograms were used to assess whether continuous variables were normally distributed. Continuous data are represented as mean \pm standard deviation (SD) if normally distributed, and median \pm interquartile range (IQR) if skewed. Fisher's exact tests, Pearson Chi square tests, independent sample t-tests and Mann-Whitney $U$ tests were used to assess group differences. Binary logistic regression analysis was used to assess the association between low SMM and the occurrence of PCF. Kaplan Meier estimates were used to visualize the relationship between low SMM and survival outcomes. Univariate and multivariate Cox proportional hazard models were used to assess the association between low SMM and overall survival. Parameters entered as covariates in regression analysis were chosen based on known or expected association with outcomes. All analyses were two-sided and $p \leq 0.05$ was considered significant.

\section{Results}

Between January 2008 and June 2017, 245 patients underwent TL at ou institution. Of these 245 patients, 235 (95.9\%) had appropriate imaging available and were included in this study. Mean interval between imaging and TL was 3 weeks; maximum was 3 months. Four patients without recent imaging available that were excluded from this study were diagnosed with a dysfunctional larynx and underwent functional laryngectomy, 2 patients had severe dental artefacts on the imaging impeding accurate SMM assessment at the level of $\mathrm{C} 3$, and 4 patients had irretrievable scans.

\section{Patient demographics}

Patient, disease and surgical characteristics are presented in Table 1, and outcomes after surgery in Table 2. During the study period, 108 patients underwent primary $\mathrm{TL}, 114$ patients underwent salvage TL and 13 patients underwent a functional TL. A complication of any grade occurred in $64.3 \%$ of patients after TL. The most commonly noted complication was a Clavien Dindo grade I transient hypocalcaemia after surgery, necessitating short-term calcium monitoring and supplementation. Severe complications occurred in 69 patients (29.4\%). A PCF occurred in 64 patients (27.2\%), which required surgical closure in 40 patients (17.0\%). Mean duration of hospital stay after TL was 21 days. There were 4 postoperative deaths (1.7\%). At the time of concluding this study, with median time of 62.7 months after TL, 134 (57.0\%) patients had died of any cause and 101 (43.0\%) were alive. 
Table 1: Patient, disease and surgical characteristics

\begin{tabular}{|c|c|c|c|c|}
\hline Characteristic & $\begin{array}{l}\text { All patients } \\
(\mathrm{n}=235)\end{array}$ & $\begin{array}{l}\text { Patients with low } \\
\text { SMM }(n=109) \\
\end{array}$ & $\begin{array}{l}\text { Patients with normal SMM } \\
(\mathrm{n}=126)\end{array}$ & p-value \\
\hline \multicolumn{5}{|l|}{ Sex } \\
\hline $\begin{array}{l}\text { Male } \\
\text { Female }\end{array}$ & $\begin{array}{l}193(82.1) \\
42(17.9)\end{array}$ & $\begin{array}{c}72(66.1) \\
37(33.9 \%)\end{array}$ & $\begin{array}{l}121(96.0) \\
5(4.0)\end{array}$ & $<0.001^{c}$ \\
\hline Age & $64.7(9.1)$ & $65.6(8.9)$ & $63.8(9.3)$ & $0.132^{d}$ \\
\hline BMI & $23.9(5.0)$ & $21.1(3.8)$ & $26.4(4.6)$ & $<0.001^{d}$ \\
\hline \multicolumn{5}{|l|}{ Smoking } \\
\hline Never & $6(2.6)$ & $4(3.7)$ & $2(1.6)$ & $0.043^{e}$ \\
\hline Current & $112(47.6)$ & $60(55.0)$ & $52(41.3)$ & \\
\hline Former & $117(49.8)$ & $45(41.3)$ & $72(57.1)$ & \\
\hline \multicolumn{5}{|l|}{ Alcohol abuse } \\
\hline No & $159(67.7)$ & $72(66.0)$ & $87(69.0)$ & $0.718^{e}$ \\
\hline Yes, current & $26(11.1)$ & $14(12.8)$ & $12(9.5)$ & \\
\hline Yes, former & $50(21.3)$ & $23(21.1)$ & $27(21.4)$ & \\
\hline \multicolumn{5}{|l|}{ ASA classification } \\
\hline 1 & $19(8.1)$ & $7(6.4)$ & $12(9.5)$ & $0.225^{\mathrm{e}}$ \\
\hline$\|$ & $109(46.4)$ & $46(42.2)$ & $63(50.0)$ & \\
\hline III & $107(45.5)$ & $56(51.4)$ & $51(40.5)$ & \\
\hline \multicolumn{5}{|l|}{ Localisation tumour } \\
\hline Larynx & $175(74.2)$ & $75(68.8)$ & $99(78.6)$ & $0.089^{e}$ \\
\hline Hypopharynx & $61(25.8)$ & $34(31.2)$ & $27(21.4)$ & \\
\hline \multicolumn{5}{|l|}{ T classification } \\
\hline TO & $13(5.5)$ & $10(9.2)$ & $3(3.2)$ & $0.008^{e}$ \\
\hline Т1-2 & $75(31.9)$ & 2623.9) & $49(38.9)$ & \\
\hline T 3-4 & $147(62.6)$ & $73(67.0)$ & $74(57.9)$ & \\
\hline \multicolumn{5}{|l|}{$\mathrm{N}$ classification } \\
\hline No & $152(64.7)$ & $64(58.7)$ & $88(69.8)$ & $0.197^{\mathrm{e}}$ \\
\hline N1 & $17(7.2)$ & $7(6.4)$ & $10(7.9)$ & \\
\hline N2 & $64(27.2)$ & $37(33.9)$ & $27(21.4)$ & \\
\hline N3 & $2(0.9)$ & $1(0.9)$ & $1(0.8)$ & \\
\hline \multicolumn{5}{|l|}{ AJCC-stage $^{a}$} \\
\hline 0 & $13(6.0)$ & $10(9.2)$ & $3(2.4)$ & $0.009 e^{e}$ \\
\hline 1 & $28(11.9)$ & $6(5.5)$ & $22(17.5)$ & \\
\hline$\|$ & $35(14.9)$ & $16(14.7)$ & $19(15.1)$ & \\
\hline III & $35(14.9)$ & $14(12.8)$ & $21(16.7)$ & \\
\hline IV & $124(52.8)$ & $63(57.8)$ & $61(48.4)$ & \\
\hline \multicolumn{5}{|l|}{ Indication for TL } \\
\hline Primary HNC & $108(46.0)$ & $57(52.3)$ & $51(40.5)$ & $0.004^{e}$ \\
\hline Recurrent/residual HNC & $114(48.5)$ & $42(38.5)$ & $72(57.1)$ & \\
\hline Dysfunctional larynx & $13(5.5)$ & $10(9.2)$ & $3(2.4)$ & \\
\hline \multicolumn{5}{|l|}{ Prior treatment } \\
\hline None & $106(45.1)$ & $55(50.5)$ & $51(40.5)$ & $0.304^{e}$ \\
\hline Radiotherapy & $106(45.1)$ & $44(40.4)$ & $62(49.2)$ & \\
\hline Chemoradiotherapy & $23(9.8)$ & $10(9.2)$ & $13(10.3)$ & \\
\hline \multicolumn{5}{|l|}{ Type of resection } \\
\hline Laryngectomy (LE) & $159(67.6)$ & $66(60.6)$ & $93(73.8)$ & $0.036^{\mathrm{e}}$ \\
\hline LE + pharyngectomy & $76(32.4)$ & $43(39.4)$ & $33(26.2)$ & \\
\hline
\end{tabular}

\begin{tabular}{lcccc}
\hline $\begin{array}{l}\text { Closure of neopharynx } \\
\text { Vertical }\end{array}$ & $129(54.9)$ & $49(45.0)$ & $80(63.5)$ & $0.117^{\mathrm{e}}$ \\
$\quad$ T-closure & $21(8.9)$ & $9(8.3)$ & $12(9.5)$ & \\
$\quad$ Flap closure & $85(36.2)$ & $51(46.8)$ & $34(27.0)$ & \\
\hline Lymph node dissection & & & & \\
$\quad$ None & $99(42.1)$ & $45(41.3)$ & $54(42.9)$ & $0.457^{\mathrm{e}}$ \\
Unilateral & $88(37.1)$ & $38(34.9)$ & $50(39.7)$ & \\
Bilateral & $48(20.4)$ & $26(23.9)$ & $22(17.5)$ & \\
\hline Primary flap reconstruction & & & & \\
$\quad$ No & $150(63.8)$ & $58(53.2)$ & $92(73.0)$ & 0.002 \\
Yes & $85(36.2)$ & $51(46.8)$ & $34(27.0)$ & \\
&
\end{tabular}

Continuous variables are represented as mean (standard deviation) and categorical variables are represented as number (percentage of total). Abbreviations: SMM = skeletal muscle mass; $\mathrm{BMI}=$ body mass index; ASA = American Society of Anesthesiologist's physical status classification; $\mathrm{TL}=$ total laryngectomy; $\mathrm{HNC}=$ head and neck cancer.

Before 2009: according to the 6th AJCC staging manual. After 2009: according to the $7^{\text {th }}$ AJCC staging manual.

Two patients with primary HNC underwent prior radiotherapy for non-Hodgkin lymphoma.

c Fisher's exact test.

Independent sample t-test.

Pearson Chi squared test.

\section{Body composition}

Skeletal muscle mass measurement at the level of C3 was successful in all 235 patients. The lumbar SMI was calculated from the SM area at C3 as described in the 'methods' section. Using the lumbar SMI cut-off point of $<43.2 \mathrm{~cm}^{2} / \mathrm{m}^{2}, 109$ patients (46.4\% of total) had low SMM. Patients with low SMM had a tendency to be female, had a lower BMI, had a larger primary tumour and AJCC stage, were more likely to have undergone primary $\mathrm{TL}$, and more frequently had flap reconstruction of the pharynx.

\section{Association between low SMM, postoperative complications, PCF}

\section{and hospital stay}

Table 2 shows the associations between low SMM and outcomes after TL. Patients with low SMM had more severe complications than patients with normal SMM ( $34.9 \%$ versus $24.6 \%$, difference not statistically significant, $p=0.114)$. All 4 patients who died in-hospital had low SMM ( $p=0.045)$. PCF occurred significantly more often in patients with low SMM than in patients with normal SMM $(p=0.019)$, and surgical treatment of the PCF was more often necessary $(p=0.050)$. Hospital stay was significantly longer in patients with low SMM (median 17 days versus 14 days, $\mathrm{p}<0.001)$. 
Table 2: Short- and long-term outcomes after TL

\begin{tabular}{|c|c|c|c|c|}
\hline Outcome & $\begin{array}{l}\text { All patients } \\
(\mathrm{n}=235)\end{array}$ & $\begin{array}{l}\text { Patients with low } \\
\text { SMM }(n=109)\end{array}$ & $\begin{array}{l}\text { Patients with normal SMM } \\
\qquad(\mathrm{n}=126)\end{array}$ & $\begin{array}{l}\text { P-value } \\
\end{array}$ \\
\hline All grade complications & $151(64.3)$ & $74(67.9)$ & $77(61.1)$ & $0.377^{\mathrm{b}}$ \\
\hline Severe complications ${ }^{a}$ & $69(29.4)$ & $38(34.9)$ & 31 (24.6) & $0.114^{b}$ \\
\hline Postoperative mortality & $4(1.7)$ & $4(3.7)$ & $0(0)$ & $0.045^{b}$ \\
\hline PCF & $64(27.2)$ & $38(34.9)$ & $26(20.6)$ & $0.019^{b}$ \\
\hline \multicolumn{5}{|l|}{ Treatment PCF } \\
\hline Conservative & $24(10.2)$ & $14(12.8)$ & $10(7.9)$ & $0.050 \mathrm{c}$ \\
\hline Surgical & $40(17.0)$ & $24(22.0)$ & $16(12.7)$ & \\
\hline Duration of hospital stay in days & $14[13-21]$ & $17[13-28]$ & $14[12-17]$ & $<0.001^{\mathrm{d}}$ \\
\hline \multicolumn{5}{|l|}{ Overall survival after TL } \\
\hline Alive & $101(43.0)$ & $30(27.5)$ & $71(56.3)$ & $<0.001^{b}$ \\
\hline Deceased & $134(57.0)$ & $79(72.5)$ & $55(43.7)$ & \\
\hline
\end{tabular}

Duration of hospital stay was skewed and is represented as median [interquartile range (IQR)] Categorical variables are represented as number and percentage of total.

aSevere complications: Clavien-Dindo Classification of Surgical Complications grade 3A or higher

Fisher's exact test

'Pearson Chi squared test

Mann-Whitney U test

Logistic regression analysis was performed to identify predictors of the occurrence of PCF. Variables were selected for logistic regression based on known or hypothesized association with PCF. T-stage and AJCC stage were not entered in logistic regression analysis because of interaction with the indication for TL. Results of the univariate and multivariate logistic regression analysis are shown in Table 3. In univariate logistic regression analysis, localisation of the tumour (hypopharynx), the type of resection (laryngectomy + pharyngectomy), flap closure of the pharynx, low SMM and a dysfunctional larynx as the indication for TL were significant predictors for PCF. In multivariate logistic regression analysis, only a hypopharyngeal tumour (OR 3.348, 95\% Cl $1.740-6.443$ ), low SMM (OR $1.950,95 \% \mathrm{Cl} 1.038$ - 3.664) and a dysfunctional larynx (OR 4.881, 95\% Cl 1.375 17.325) remained significant predictors of PCF.
Table 3: Univariate \& multivariate logistic regression analysis for the occurrence of PCF

\begin{tabular}{|c|c|c|c|c|}
\hline \multirow{2}{*}{$\begin{array}{l}\text { Risk factor } \\
\text { nat }\end{array}$} & \multicolumn{2}{|l|}{ Univariate analysis ${ }^{\mathrm{a}}$} & \multicolumn{2}{|c|}{ Multivariate analysis ${ }^{b}$} \\
\hline & Odds ratio (95\% Cl) & $p$-value & Odds ratio (95\% Cl) & $\mathrm{p}$-value \\
\hline \multicolumn{5}{|l|}{ Sex } \\
\hline Male & 1.00 [reference] & & & \\
\hline Female & $1.633(0.803-3.319)$ & 0.176 & $1.091(4.72-2.521)$ & 0.839 \\
\hline \multicolumn{5}{|l|}{ Smoking } \\
\hline Never & 1.00 & & & \\
\hline Current & $1.747(0.196-15.583)$ & 0.520 & & \\
\hline Former & $2.048(0.231-18.188)$ & 0.617 & & \\
\hline \multicolumn{5}{|l|}{ Alcohol abuse } \\
\hline Never & 1.00 & & & \\
\hline Yes, current & $1.322(0.534-3.274)$ & 0.546 & & \\
\hline Yes, former & $1.400(0.700-2.802)$ & 0.342 & & \\
\hline \multicolumn{5}{|l|}{ Prior treatment } \\
\hline None & 1.00 & & & \\
\hline Radiotherapy & $1.330(0.725-2.473)$ & 0.351 & & \\
\hline Chemoradiotherapy & $1.728(0.656-4.550)$ & 0.268 & & \\
\hline \multicolumn{5}{|l|}{ Localisation tumour } \\
\hline Larynx & 1.00 & & & \\
\hline Hypopharynx & $3.276(1.757-6.107)$ & & $3.348(1.740-6.443)$ & $<0.001$ \\
\hline \multicolumn{5}{|l|}{ Type of resection } \\
\hline Laryngectomy (LE) & 1.00 & & & \\
\hline $\mathrm{LE}+$ pharyngectomy & $3.003(1.646-5.478)$ & $<0.001$ & $1.799(0.852-3.798)$ & 0.123 \\
\hline \multicolumn{5}{|l|}{ Closure of neopharynx } \\
\hline Vertical & 1.00 & & & \\
\hline T-closure & $1.492(0.496-4.489)$ & 0.181 & $1.581(0.511-4.896)$ & 0.427 \\
\hline Flap closure & $3.238(1.734-6.047)$ & 0.001 & $1.580(0.525-4.753)$ & 0.416 \\
\hline \multicolumn{5}{|l|}{ LND } \\
\hline None & 1.00 & & & \\
\hline Unilateral & $1.458(0.767-2.772)$ & 0.250 & & \\
\hline Bilateral & $1.042(0.469-2.316)$ & 0.920 & & \\
\hline \multicolumn{5}{|l|}{ Indication for TL } \\
\hline Primary HNC & 1.00 & & & \\
\hline Recurrent/residual HNC & $1.126(0.614-2.067)$ & 0.701 & $1.560(0.809-3.011)$ & 0.185 \\
\hline Dysfunctional larynx & $5.046(1.518-16.775)$ & 0.008 & $4.881(1.375-17.325)$ & 0.014 \\
\hline \multicolumn{5}{|l|}{ Low BMI } \\
\hline$<18.5$ & $1.191(0.373-3.801)$ & 0,768 & & \\
\hline $18.5-25.0$ & $0.935(0.374-2.340)$ & 0,886 & & \\
\hline $25.0-30.0$ & $0.479(0.163-1.405)$ & 0.180 & & \\
\hline$>30.0$ & 1.00 & & & \\
\hline Low SMM & $2.059(1.148-3.692)$ & 0.015 & $1.950(1.038-3.664)$ & 0.038 \\
\hline
\end{tabular}

Univariate binary logistic regression analysis

Multivariate binary logistic regression (Backward Wald selection model)

LND = lymph node dissection 


\section{Survival analysis}

On univariate analysis, OS at the end of follow-up was significantly lower in patients with low SMM than in patients with normal SMM (median OS was 18.5 months in patients with low SMM versus 30.1 months in patients with normal SMM, $p<0.001$ ), which is visualized in Figure 2. Disease specific survival was significantly lower in patients with low SMM as well as shown in Figure $\mathbf{3}$

Table 4 shows the results of the univariate and multivariate regression analysis for OS. In univariate Cox proportional hazard regression analysis, high $\mathrm{N}$-stage (N2 and N3) and low SMM were significant prognosticators for OS. A higher BM appeared to be associated with longer OS. In multivariate Cox regression analysis, N2 nodal status (OR 1.494, 95\% Cl $1.023-2.182, \mathrm{p}=0.038)$, N3 nodal status (OR $16.040,95 \% \mathrm{Cl} 3.691-69.710, \mathrm{p}<0.001)$ and low SMM (OR 1.849 (1.202-2.843) $p=0.005)$ remained independent prognosticators for decreased OS.

\begin{tabular}{|c|c|c|c|c|}
\hline \multirow[t]{2}{*}{ Risk factor } & \multirow{2}{*}{$\begin{array}{l}\text { Univariate analysis } \\
\text { Odds ratio }(95 \% \mathrm{Cl})\end{array}$} & \multicolumn{3}{|c|}{ Multivariate analysis $^{b}$} \\
\hline & & p-value & Odds ratio $(95 \% \mathrm{Cl})$ & $p$-value \\
\hline \multicolumn{5}{|l|}{ Sex } \\
\hline Male & 1.00 & & & \\
\hline Female & $0.940(0.603-1.465)$ & 0.784 & & \\
\hline \multicolumn{5}{|l|}{ Indication for TL } \\
\hline Primary HNC & 1.00 [reference] & & & \\
\hline Recurrent/residual HNC & $1.308(0.923-1.855)$ & 0.131 & & \\
\hline Dysfunctional larynx & $1.017(0.439-2.355)$ & 0.969 & & \\
\hline \multicolumn{5}{|l|}{$N$ classification ${ }^{c}$} \\
\hline No & 1.00 & & & \\
\hline N1 & $1.771(0.979-3.201)$ & 0.059 & $1.452(0.783-2.692)$ & 0.237 \\
\hline N2 & $1.763(1.214-2.560)$ & 0.004 & $1.313(0.892-1.932)$ & 0.167 \\
\hline N3 & $17.091(3.939-74.153)$ & $<0.001$ & $17.4170(3.919-77.410)$ & $<0.001$ \\
\hline \multicolumn{5}{|l|}{ Postoperative treatment } \\
\hline None & 1.00 & & & \\
\hline Radiotherapy & $0.756(0.524-1.092)$ & 0.136 & & \\
\hline Chemoradiotherapy & $0.834(0.461-1.509)$ & 0.549 & & \\
\hline \multicolumn{5}{|l|}{ ASA classification } \\
\hline 1 & 1.00 & & & \\
\hline ॥ & $1.286(0.638-2.590)$ & 0.482 & & \\
\hline III & $1.746(0.868-3.511)$ & 0.118 & & \\
\hline \multicolumn{5}{|l|}{ Low BMI } \\
\hline$<18.5$ & $1.237(0.605-2.528)$ & 0.560 & $0.710(0.332-1.568)$ & 0.397 \\
\hline $18.5-25.0$ & $1.193(0.662-2.150)$ & 0.556 & $0.904(0.487-1.679)$ & 0.750 \\
\hline $25.0-30.0$ & $0.494(0.246-0.989)$ & 0.047 & $0.538(0.267-1.081)$ & 0.082 \\
\hline$>30.0$ & 1.00 & & & \\
\hline Low SMM & $2.217(1.566-3.137)$ & $<0.001$ & $1.849(1.202-2.843)$ & 0.005 \\
\hline
\end{tabular}

Univariate Cox survival regression analysis

Multivariate Cox survival regression analysis (Backward Wald selection model)

Patients with $\mathrm{N} 3$ nodal status: $\mathrm{n}=2$.
Figure 2: Overall survival after TL
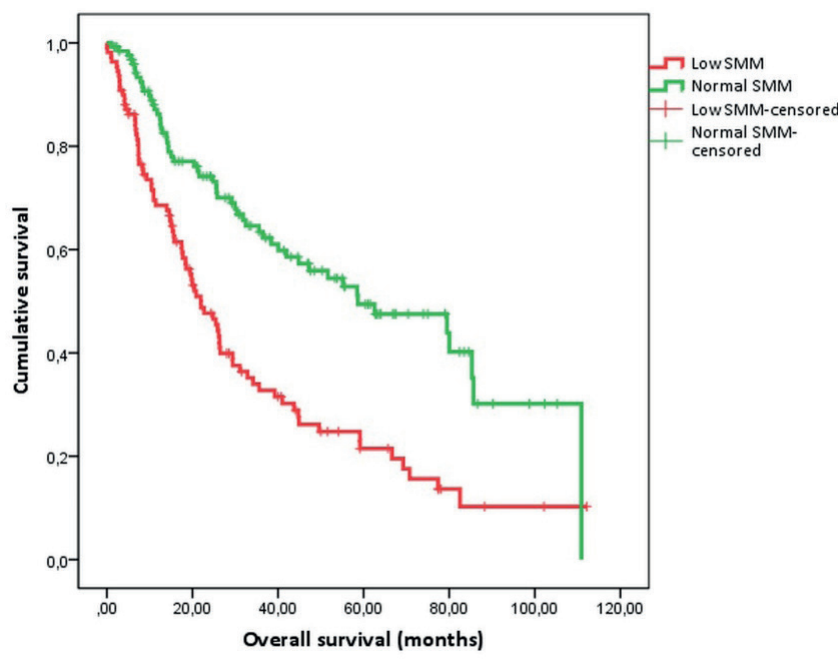

Figure 3: Disease specific survival after TL
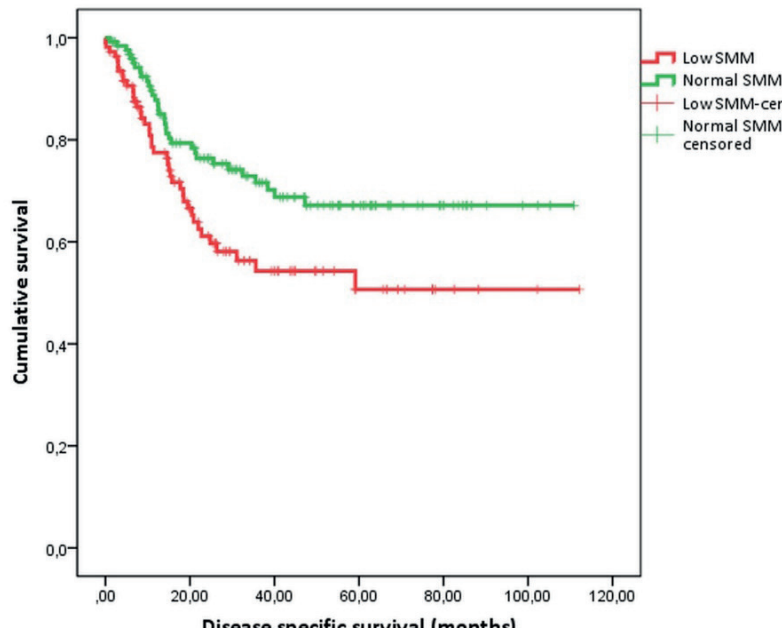
LowSMM-censored
Normal SMM-
censored

Kaplan Meier survival curve showing overall survival in patients with low and normal SMM. The median OS for patients with low SMM after laryngectomy was 18.5 months, compared to 30.1 months in patients with normal SMM $(p<0.001)$. Overall survival at 5 -years after TL was $32.1 \%$ for patients with low SMM versus $61.1 \%$ for patients with normal SMM (log rank test: $p<0.001)$. 
Kaplan Meier survival curves showing disease specific survival in patients with low and normal SMM. Disease specific survival (DSS) and 5-year DSS were equal; DSS was $63.3 \%$ for patients with low SMM versus $73.8 \%$ for patients with normal SMM (log rank test: $\mathrm{p}=0.019$ ).

\section{Discussion}

It can be anticipated that low SMM has a high prevalence in head and neck cance patients, at least partly due to the location of the tumor and/or poor physical condition of head and neck cancer patients. ${ }^{22,23}$ Many head and neck cancer patients experience dysphagia and odynophagia, especially after initial (chemo) radiotherapy, leading to malnutrition at diagnosis. ${ }^{24,25} \mathrm{~A}$ recent study in patients with advanced stage head and neck squamous cell carcinoma undergoing primary chemoradiotherapy, in which SMM was measured at the level of C3, found that $54.5 \%$ of patients had low SMM prior to start of treatment. ${ }^{21}$ Another study, in which SMM was measured at the level of L3, found that $77 \%$ of patients undergoing TL had low SMM. ${ }^{26}$ In the latter study, low SMM was a significant independent predictor for the occurrence of PCF. ${ }^{26}$ One shortcoming of this study was that abdominal imaging was available in only $57 \%$ of patients and consequently only in these patients SMM could be assessed. Because it might be expected that whole body imaging is only routinely performed in selected patients, e.g. with advanced stage disease and at high risk of distant metastases, a substantial risk of bias has been introduced.

This study is the largest to date in head and neck cancer patients to show that low SMM is a powerful negative prognostic factor in patients undergoing primary salvage or functional TL. Patients with low SMM are at increased risk of developing PCF after TL compared to patients with normal SMM, and the overall survival of patients with low SMM is only half of the overall survival of patients with normal SMM. Our data suggest that a simple preoperative measurement of SMM may aid in identifying patients at risk for severe complications, in particular of PCF, and prolonged hospital stay. It may also function as a strong negative prognostic parameter for overall survival after TL.

Our study is the first to use routinely performed CT or MRI imaging of the head and neck area to assess preoperative muscle status in patients undergoing TL, and the largest to do so in head and neck cancer patients. The main benefit of SMM measurement at the level of $\mathrm{C} 3$, compared to SMM measurement at the level of
L3, is that almost all patients have appropriate imaging available. In this current study, only 10 out of 245 (4.1\%) patients had to be excluded. ${ }^{26}$

The relationship between low SMM and negative short-term outcomes and prolonged hospital stay is consistent with previous publications in surgical oncology. In abdominal surgery, a recent systematic review and meta-analysis showed that low SMM is a significant risk factor for major postoperative complications, postoperative mortality and shorter $1-, 3-$, and 5-year survival. ${ }^{15}$ In hepatopancreatobiliary cancer, low SMM is associated with reduced overall survival. ${ }^{14}$ In patients with resectable early stage non-small cell lung cancer, low SMM is associated with poor short- and long-term outcomes after surgery. 27,28 Low SMM has also been associated with prolonged hospital stay and increased healthcare related costs. A recent study in 452 patients undergoing abdominal surgery for cancer of the alimentary tract showed that patients with low SMM had a significantly longer hospital stay and significantly higher hospital costs than patients with normal SMM..$^{29}$ In our study, patients with low SMM had a significantly longer hospital stay than patients with normal SMM. Although not investigated, it can be anticipated from our results that healthcare related costs have been higher in patients with low SMM as well. Our study also showed a clear relationship with poor overall survival and disease-specific survival in patients with low SMM. This is in line with previous publications in other types of cancer. ${ }^{3}$ This may be due to patients not being fit enough to receive adjuvant treatment or severe postoperative complications resulting in mortality or delaying necessary adjuvant treatment.

In our study, a cut-off value to define low SMM that has been developed in a separate cohort of Dutch head and neck cancer patients was used. ${ }^{21}$ Although exact definitions and cut-off values for low SMM differ between studies, patient groups and ethnicities, it does appear that low SMM is consistently associated with adverse short-term and long-term outcomes in surgical oncology. A potential hypothesis for this may be that patients with low SMM have a decreased capability for recovery after major cancer surgery, for instance due to an altered protein metabolism or a decreased physiological reserve to deal with surgical stress. ${ }^{31,32}$ Another hypothesis is that low SMM indirectly reflects an overall poorer physical functioning in patients, which is not as distinctly found using other routinely used risk stratification methods.

Future research should be aimed at proactive interventions to improve patient's physical and nutritional status, to clarify whether the adverse effects 
of low SMM are prognostic only, or if they can be overturned by intensive preoperative optimization and postoperative rehabilitation with physical therapy and nutritional support. Feasibility studies in head and neck cancer patients have shown that resistance training programs in patients undergoing chemoradiotherapy or radiotherapy are feasible and show high patient satisfaction. ${ }^{33,34} \mathrm{~A}$ randomized controlled trial in lung cancer patients undergoing short-term intensive rehabilitation prior to radical surgery showed positive results, with a significant decrease in hospital stay after surgery. ${ }^{35}$ However, most current trials are small; larger randomized controlled trials should clarify whether a multimodal rehabilitation program can reduce the negative effects of low SMM in patients undergoing TL. Alternatively, treatment planning could take the low SMM into account. For example, this could lead the head and neck surgeon to more frequently use overlay pectoralis major flaps for reinforcement in order to decrease the risk of PCF in patients at high risk of PCF. ${ }^{36}$ In our cohort, an overlay pectoralis major flap was only used in 9 patients; as such, no conclusions could be drawn of its protective effect.

There are some limitations of our study to discuss. The cut-off value that we used to define low SMM in patients is not a sex-specific cut-off value. We do believe that a sex-specific cut-off value would be superior to the cut-off value currently used. However, particularly in female patients we currently do not have enough data to reliably formulate sex-specific cut-offs for head and neck cancer patients. We also evaluated using other published, sex-specific and BMI-specific cutoffs for low SMM, which have been formulated in large cohorts of patients with different types of cancer. ${ }^{12,37}$ Using these cut-offs, approximately $80 \%$ of patients are classified as having low SMM. Thus, these cut-offs seem to lose their clinical discriminative power in head and neck cancer patients.

We found some discrepancies between the results of the recent Dutch Head and Neck Society Audit for risk factors of PCF after TL, and our current research. ${ }^{7}$ For instance, previous chemoradiotherapy was a risk factors for PCF occurrence after TL in the Dutch Head and Neck Society Audit, and it was not a significant predictor for PCF in our cohort. This may be explained by the fact that relatively few patients in our cohort had had chemoradiotherapy before TL $(9.8 \%$ in our cohort compared to $15.6 \%$ in the Dutch Head and Neck Society cohort). As we did find a slightly higher albeit non-significant risk of PCF in patients who had had chemoradiotherapy prior to $\mathrm{TL}$, we believe that our analysis was underpowered for this risk factor.
Due to the retrospective nature of the research, all relevant research parameters for body composition or nutritional status may not have been documented or measured during normal clinical practice. In this study, this was particularly true for the plasma albumin level, which was often not measured in our cohort of patients undergoing TL. Also, the ASA classification for physical status was used as a surrogate marker for representing comorbidity. Unfortunately, more specific comorbidity scales could not be determined in this retrospective study because of missing information, particularly in the first 3 years of the study period. Muscle function was not assessed in this study, as muscle function is not tested during routine clinical practice in our institution. Possibly, a measurement of muscle mass and muscle function and/or physical condition (following the geriatric definition of sarcopenia ${ }^{10}$ ) may provide a more accurate risk profile of muscular status in patients undergoing TL than CT or MRI measured muscle mass alone.

Concerning the imaging techniques used to assess SMM, we decided to include both CT scans and MRI scans of the head and neck area to assess SMM, in order to maximize the number of patients that could be included. Whenever available, we used CT imaging instead of MRI because most research on SMM in cancer patients is performed using CT imaging. However, the CT measurement method for SMM was formulated on MRI-based research. ${ }^{12,13}$ Theoretically there is no difference in SMM between CT imaging and MRI, as both methods are very accurate for SMM assessment. Therefore, we believe it is acceptable to use MRI for SMM measurement when CT imaging is not available. Also, a measurement of SM density, which is the mean HU value of the SM area, was not included in this analysis. A recent study showed that SM area does not differ significantly between CT with and without contrast, but SM density does differ significantly. ${ }^{38}$ As we used both CT with and without contrast for measurement of SMM, we decided not to include SM density in this analysis.

\section{Conclusion}

In this study, we found that preoperative low SMM is statistically associated with more frequent $\mathrm{PCF}$, prolonged hospital stay and reduced overall survival in patients undergoing TL for any indication. A measurement of SMM at the level of 3 allows for routine SMM assessment in diagnostic imaging, without the need for additional abdominal imaging. Our results advocate a preoperative assessment of SMM in patients undergoing TL to identify high risk patients. Possibly, intensive prehabilitation strategies aimed at increasing SMM may reduce these negative outcomes, or wider use of flap reconstruction in patients with low SMM may prevent complications from occurring. Prospective research is needed to evaluate this. 


\section{References}

1. van der Putten L, de Bree R, Kuik DJ, Rietveld DH, Buter J, Eerenstein SE, et al. Salvage laryngectomy: oncological and functional outcome. Oral Oncol. 2011;47(4):296-301.

2. Theunissen EA, Timmermans AJ, Zuur CL, Hamming-Vrieze O, Paul de Boer J, Hilgers FJ, et al. Total laryngectomy for a dysfunctional larynx after (chemo)radiotherapy. Arch Otolaryngo Head Neck Surg. 2012;138(6):548-55.

3. Paydarfar JA, Birkmeyer NJ. Complications in head and neck surgery: a meta-analysis of postlaryngectomy pharyngocutaneous fistula. Arch Otolaryngol Head Neck Surg. 2006;132(1):67-72.

4. Goepfert RP, Hutcheson KA, Lewin JS, Desai NG, Zafereo ME, Hessel AC, et al. Complications, hospital length of stay, and readmission after total laryngectomy. Cancer. 2017;123(10):1760-7. Hasan Z, Dwivedi RC, Gunaratne DA, Virk SA, Palme CE, Riffat F. Systematic review and metaanalysis of the complications of salvage total laryngectomy. Eur J Surg Oncol. 2017;43(1):42-51 Ganly I, Patel S, Matsuo J, Singh B, Kraus D, Boyle J, et al. Postoperative complications of salvage total laryngectomy. Cancer. 2005;103(10):2073-81.

7. Lansaat L, van der Noort V, Bernard SE, Eerenstein SEJ, Plaat BEC, Langeveld T, et al. Predictive factors for pharyngocutaneous fistulization after total laryngectomy: a Dutch Head and Neck Society audit. Eur Arch Otorhinolaryngol 2018 Mar;275(3):783-794

8. Carneiro IP, Mazurak VC, Prado CM. Clinical Implications of Sarcopenic Obesity in Cancer. Curr Oncol Rep. 2016;18(10):62.

9. Ryan AM, Power DG, Daly L, Cushen SJ, Ni Bhuachalla E, Prado CM. Cancer-associated malnutrition, cachexia and sarcopenia: the skeleton in the hospital closet 40 years later. Proc Nutr Soc. 2016;75(2):199-211

10. Cruz-Jentoft AJ, Baeyens JP, Bauer JM, Boirie Y, Cederholm T, Landi F, et al. Sarcopenia: European consensus on definition and diagnosis: Report of the European Working Group on Sarcopenia in Older People. Age and ageing. 2010;39(4):412-23.

11. Rier HN, Jager A, Sleijfer S, Maier AB, Levin MD. The Prevalence and Prognostic Value of Low Muscle Mass in Cancer Patients: A Review of the Literature. Oncologist. 2016 Nov:21(11):13961409. 12. Prado CM, Lieffers JR, McCargar LJ, Reiman T, Sawyer MB, Martin L, et al. Prevalence and clinical implications of sarcopenic obesity in patients with solid tumours of the respiratory and gastrointestinal tracts: a population-based study. Lancet Oncol. 2008;9(7):629-35.

13. Shen W, Punyanitya M, Wang Z, Gallagher D, St-Onge MP, Albu J, et al. Total body skeleta muscle and adipose tissue volumes: estimation from a single abdominal cross-sectional image. muscle and ad (1985). 2004:97(6):2333-8.

14. Levolger S, van Vugt JL, de Bruin RW, IJzermans JNM. Systematic review of sarcopenia in patients operated on for gastrointestinal and hepatopancreatobiliary malignancies. Br I Surg. 2015;102(12):1448-58.

15. Jones K, Gordon-Weeks A, Coleman C, Silva M. Radiologically Determined Sarcopenia Predicts Morbidity and Mortality Following Abdominal Surgery: A Systematic Review and MetaAnalysis. World J Surg. 2017 Sep;41(9):2266-2279.

16. Swartz JE, Pothen AJ, Wegner I, Smid EJ, Swart KM, de Bree R, et al. Feasibility of using head and neck CT imaging to assess skeletal muscle mass in head and neck cancer patients. Oral Oncol. 2016;62:28-33.

17. Edge SB, Compton CC. The American Joint Committee on Cancer: the 7th edition of the AJCC cancer staging manual and the future of TNM. Ann Surg Oncol. 2010 Jun;17(6):1471-4.

18. Hurwitz EE, Simon M, Vinta SR, Zehm CF, Shabot SM, Minhajuddin A, et al. Adding Examples to the ASA-Physical Status Classification Improves Correct Assignment to Patients. Anesthesiology. 2017 Apr;126(4):614-622.

19. Dindo D, Demartines N, Clavien PA. Classification of surgical complications: a new proposal with evaluation in a cohort of 6336 patients and results of a survey. Ann Surg. 2004 Aug;240(2):20513.

20. Heymsfield SB, Wang Z, Baumgartner RN, Ross R. Human body composition: advances in models and methods. Annu Rev Nutr. 1997; 17:527-58.
21. Wendrich AW, Swartz JE, Bril SI, Wegner I, de Graeff A, Smid EJ, et al. Low skeletal muscle mass is a predictive factor for chemotherapy dose-limiting toxicity in patients with locally advanced head and neck cancer. Oral Oncol. 2017 Aug:71:26-33.

22. Argiris A, Karamouzis MV, Raben D, Ferris RL. Head and neck cancer. Lancet. 2008 May 17:371(9625):1695-709.

23. Hebuterne X, Lemarie E, Michallet M, de Montreuil CB, Schneider SM, Goldwasser F. Prevalence of malnutrition and current use of nutrition support in patients with cancer. JPEN J Parenter Enteral Nutr. $2014 \mathrm{Feb} ; 38(2): 196-204$.

24. Jager-Wittenaar H, Dijkstra PU, Dijkstra G, Bijzet J, Langendijk JA, van der Laan B, et al. High prevalence of cachexia in newly diagnosed head and neck cancer patients: An exploratory study. Nutrition. 2017 Mar;35:114-118.

25. Jager-Wittenaar $\mathrm{H}$, Dijkstra PU, Vissink A, van Oort RP, van der Laan BF, Roodenburg JL Malnutrition in patients treated for oral or oropharyngeal cancer--prevalence and relationsh with oral symptoms: an explorative study. Support Care Cancer. 2011 Oct;19(10):1675-83.

26. Achim V, Bash J, Mowery A, Guimaraes AR, Li R, Schindler J, et al. Prognostic Indication of Sarcopenia for Wound Complication After Total Laryngectomy. JAMA Otolaryngol Head Neck Surg. 2017 Dec 1;143(12):1159-1165.

27. Suzuki Y, Okamoto T, Fujishita T, Katsura M, Akamine T, Takamori S, et al. Clinical implication of sarcopenia in patients undergoing complete resection for early non-small cell lung cancer. Lung Cancer. 2016 Nov; 101:92-97.

28. Tsukioka T, Nishiyama N, Izumi N, Mizuguchi S, Komatsu H, Okada S, et al. Sarcopenia is a nove poor prognostic factor in male patients with pathological Stage I non-small cell lung cancer. Jpn J Clin Oncol. 2017 Apr 1;47(4):363-368.

29 van Vugt JLA, Buettner S, Levolger S, Coebergh van den Braak RRJ, Suker M, Gaspersz MP, et al. Low skeletal muscle mass is associated with increased hospital expenditure in patients undergoing cancer surgery of the alimentary tract. PLoS One. 2017 Oct 31;12(10):e0186547.

30 Shachar SS, Williams GR, Muss HB, Nishijima TF. Prognostic value of sarcopenia in adults with solid tumours: A meta-analysis and systematic review. Eur J Cancer. 2016 Apr;57:58-67.

31 Tew GA, Ayyash R, Durrand J, Danjoux GR. Clinical guideline and recommendations on preoperative exercise training in patients awaiting major non-cardiac surgery. Anaesthesia. 2018 Jun; 73(6):750-768

32. Buford TW, Anton SD, Judge AR, Marzetti E, Wohlgemuth SE, Carter CS, et al. Models of accelerated sarcopenia: critical pieces for solving the puzzle of age-related muscle atrophy. Ageing Res Rev. 2010 Oct;9(4):369-83.

33. Sandmael JA, Bye A, Solheim TS, Stene GB, Thorsen L, Kaasa S, et al. Feasibility and preliminary effects of resistance training and nutritional supplements during versus after radiotherapy in patients with head and neck cancer: A pilot randomized trial. Cancer. 2017 Nov 15;123(22):44404448 .

34. Brown TE, Banks MD, Hughes BGM, Lin CY, Kenny LM, Bauer JD. Randomised controlled trial of early prophylactic feeding vs standard care in patients with head and neck cancer. Br J Cancer. 2017 Jun 27;117(1):15-24

35. Huang J, Lai Y, Zhou X, Li S, Su J, Yang M, et al. Short-term high-intensity rehabilitation in radically treated lung cancer: a three-armed randomized controlled trial. J Thorac Dis. 2017 Jul; $9(7): 1919-1929$.

36. Paleri V, Drinnan M, van den Brekel MW, Hinni ML, Bradley PJ, Wolf GT, et al. Vascularized tissue o reduce fistula following salvage total laryngectomy: a systematic review. Laryngoscope. 2014 Aug;124(8):1848-53.

37. Martin L, Birdsell L, Macdonald N, Reiman T, Clandinin MT, McCargar LJ, et al. Cancer cachexia in the age of obesity: skeletal muscle depletion is a powerful prognostic factor independent of body mass index. J Clin Oncol. 2013 Apr 20:31(12):1539-47.

38. Van Vugt JLA, Coebergh van der Braak RRJ, Schippers HJW, Veen KM, Levolger S, de Bruin RWF, t al. Contrastenhancement influences skeletal muscle density but not skeletal muscle mas, measurements on computed tomography. Clin Nutr. 2017. S0261-5614(17)30246-7. 


\section{Chapter 7}

Sarcopenia is a prognostic factor for overall survival in elderly patients with headand-neck cancer

Najiba Chargi, Sandra I. Bril, Marielle H. Emmelot-Vonk, Remco de Bree 


\section{Abstract}

Objectives Sarcopenia is known as a geriatric syndrome associated with increased disability and decreased survival in elderly patients. In oncological patients, pretreatment low skeletal muscle mass (SMM), sometimes referred to as sarcopenia, is an emerging negative prognostic factor. Commonly, only SMM is assessed in cancer patients. Sarcopenia is defined as the combination of low SMM and low muscle function (MF). We investigated the relation between SMM, MF, sarcopenia (SMM and MF combined), and overall survival (OS) in a group of elderly patients with head-and-neck squamous cell carcinoma (HNSCC).

Methods A retrospective study in elderly HNSCC patients treated between 2015 and 2018 was performed. The prognostic value of SMM and MF separately, and sarcopenia was investigated.

Results Eighty-five patients were included of whom $48.2 \%$ had sarcopenia. The median OS was significantly worse for patients treated with curative inten with sarcopenia (12.07 months; IQR 3.64-21.82) compared to patients without sarcopenia (13.60 months; IQR 5.98-27.00) (HR 2.80; 95\% Cl 1.14-6.88; $\mathrm{p}=0.03$ ). SMM and MF seperately were not significant predictors of OS.

Conclusion Sarcopenia is associated with impaired OS in elderly HNSCC patients. Sarcopenia, defined as the combination of low SMM and low MF, appears to be a better predictor of OS than low SMM or low MF separately.

\section{Introduction}

Research into the field of body composition and specifically low skeletal muscle mass (SMM), sometimes also referred to as sarcopenia, has increasingly gained interest over the last decade in the field of oncology. In geriatrics, sarcopenia is known as an age-related syndrome with a multifactorial etiology, characterized by generalized loss of SMM and loss of muscle strength. ${ }^{1}$

Risk factors for the presence of sarcopenia are malnutrition, immobilization and illness. Sarcopenia is a risk factor for various adverse outcomes including physical disability, decreased quality of life, and ultimately death. ' In human aging, muscle wasting is an imminent process. It is estimated that the prevalence of sarcopenia in the general population is $5-13 \%$ for people aged $60-70$ years, and up to $50 \%$ for those aged 80 years or above. ${ }^{2}$ Independent of age, sarcopenia is impaired in various diseases due to inflammation, malnutrition and immobilization.

The majority of research within the oncological community has defined sarcopenia as radiologically assessed low SMM and/or low skeletal muscle quality. Previous research in elderly people showed that the correlation between SMM and muscle strength is moderate to weak and the relationship between muscle strength and SMM is not linear. ${ }^{3,4}$ For this reason, the European working group on sarcopenia in older people (EWGSOP) recommended diagnosing sarcopenia based on the presence of both low SMM and low muscle function (MF; strength or performance).

Within the field of oncology, radiologically assessed low SMM appears to be a negative predictive and prognostic factor for various outcomes including disease progression and survival in a variety of cancer types. ${ }^{5}$ For example, radiologically assessed low SMM is associated with chemotherapy dose-limiting toxicity in patients with head and neck cancer ${ }^{6}$, breast cancer ${ }^{7}$ and renal cell carcinoma ${ }^{8}$; increased incidence of postoperative complications in patients with head and neck cancer9,10, esophageal squamous cell carcinoma ${ }^{11}$ and colorectal cancer ${ }^{12}$; and decreased survival in patients with head and neck cancer ${ }^{10,13}$, colorectal cancer ${ }^{14}$ and pancreatic adenocarcinoma ${ }^{15}$.

In the majority of studies on the effect of sarcopenia on survival of cancer patients, and in all studies regarding head and neck cancer patients, only radiologically assessed low SMM was used to define sarcopenia. There are very few studies available in cancer patients that assess the prognostic value of sarcopenia as 
defined by the combination of low SMM and low MF. One study performed with gastric cancer patients who underwent gastrectomy showed that patients with sarcopenia, as defined by the combination of low SMM and low MF, showed significantly higher complication rate compared to patients without sarcopenia. ${ }^{16}$ In head and neck cancer, no studies are available on the relationship between sarcopenia, as defined by the combination of low SMM and low MF, and adverse outcomes. The aim of this study is to explore the relationship between sarcopenia and overall survival in elderly patients with head and neck cancer.

\section{Methods}

\section{Patients and study design}

This study was designed as a single-center retrospective study. We reviewed elderly patients ( $\geq 70$-year old) with pathologically proven head and neck squamous cell carcinoma (HNSCC) who had a geriatric assessment during their diagnostic workup between April 2015 and February 2018. In our center elderly HNSCC patients are offered geriatric assessment, but patients may refuse. Histologic tumor types other than squamous cell carcinoma were excluded. The design of this retrospective study was approved by the Medical Ethical Research Committee of our center (approval ID 17-365/C).

Factors with known or suspected relation with HNSCC treatment outcomes were collected: age, sex, body mass index (BMI), weight loss in the past six months, risk of malnutrition assessed with the malnutrition universal screening tool (MUST), smoking status, alcohol use, comorbidity expressed as a Charlson Comorbidity ndex (CCl) score, tumor type (primary, second primary or recurrence), tumor site tumor-node-metastasis (TNM) stage and treatment intention.

\section{Definition of sarcopenia}

Sarcopenia was defined as the combination of low SMM and low MF, as determined by muscle strength or physical performance measurements.

\section{Skeletal muscle mass}

Skeletal muscle mass was measured as muscle cross-sectional muscle area (CSA) on pre-treatment $\mathrm{CT}$ or MRI imaging of the head and neck area at the level of the third cervical vertebrae (C3). The axial slide of the imaging which showed both transverse processes and the entire vertebral arc was selected for segmentation of muscle tissue. For $\mathrm{CT}$ imaging, muscle area was defined as the pixel area between the radiodensity range of -29 and +150 Hounsfield Units (HU), which is specific for muscle tissue ${ }^{17}$ For MRI, muscle area was manually segmented, and fatty tissue was manually excluded.

Segmentation of muscle tissue was manually performed using the commercially available software package SliceOmatic (Tomovision, Canada). Cross-sectiona muscle area at the level of C3 was converted to CSA at the level of L3 using previously published formula. ${ }^{18}$ The lumbar skeletal muscle index (SMI) was calculated by correcting SMM at the level of L3 for height. Patients had a low SM if this value was below $43.2 \mathrm{~cm}^{2} / \mathrm{m}^{2}$; this cutoff value was established in a separate cohort of head and neck cancer patients. ${ }^{6}$

\section{Muscle strength}

sometric handgrip strength (HGS) is strongly related with overall muscle strength. ${ }^{19}$ Handgrip strength was measured using a Jamar Hydraulic Handheld Dynamometer according to the recommendations of the American society of hand therapist's (ASHT) and expressed in kilograms (kg). Patients were asked to squeeze maximally with each hand. The average score of the left and right hand was used for analysis. Patients had low HGS if the HGS was below $30 \mathrm{~kg}$ (men) or below $20 \mathrm{~kg}$ (women).'

\section{Muscle performance}

The four-meter gait speed is a reflection of individual's lower limb muscle function. It is a widely accepted way to assess muscle performance. ${ }^{20}$ Gait speed was measured as the average speed during a four-meter walking test. The time measured to complete a four-meter walk was measured. Patients had low muscle performance if the four-meter gait speed was below $0.8 \mathrm{~m} / \mathrm{s}$.

\section{Statistical analysis}

Data analyses was performed using IBM SPSS statistics 25. Descriptive statistics for continuous variables with a normal distribution were presented as mean with standard deviation (SD). Variables with a skewed distribution were presented as median with interquartile range (IQR). Categorical variables were presented as frequencies and percentages. Pearson's correlation was used to assess the correlation between SMM, MF parameters, age and BMI. Survival was visualized using Kaplan Meier survival curves. A cox proportional hazard regression mode was used for univariate and multivariate analysis of survival. Covariates used in the multivariate analysis were selected based on clinical significance or selected based on statistical significance $(p<0.05)$ in univariate cox regression analysis. Statistical significance was evaluated at the 0.05 level using 2 -sides tests. 


\section{Results}

\section{Patient characteristics}

Descriptive data are described in Table 1. A total of 85 patients were included with a mean age of 81.5 years (SD 6.5). The majority of patients were female $(55.3 \%)$ with a mean BMI of $26.9 \mathrm{~kg} / \mathrm{m}^{2}$ (SD 4.8). Most patients were forme smokers (54.1\%) with mean pack years of 21-40 years. Most patients had multiple comorbidities, as represented by a high Charlson Comorbidity Index score (CCI). Most patients underwent treatment with curative intent (83.5\%). The median follow-up time was 11.14 months (IQR 3.64-21.83 months); 33 patients (38.8\%) died during the study period.

Of the 85 included patients; 69 patients (81.2\%) had low SMI, 50 patients $(58.8 \%)$ had low HGS and 58 patients (68.2\%) had low gait speed. Forty-one patient (48.2\%) were classified as sarcopenic; of these patients 31 patients (75.6\%) had low SMI in combination with low HGS and low gait speed, 6 patients (14.6\%) had low SMI in combination with low gait speed and normal HGS, and 4 patients (9.8\%) had low SMI in combination with low HGS and normal gait speed.

\section{Table 1. Patient characteristics}

\begin{tabular}{|c|c|c|}
\hline Characteristics & Mean (SD) & Frequencies $\mathrm{n},(\%)$ \\
\hline \multicolumn{3}{|l|}{ Sex } \\
\hline Female & & $47(55.3)$ \\
\hline Male & & $38(44.7)$ \\
\hline Age (years) & $81.5(6.5)$ & \\
\hline BMI $\left(\mathrm{kg} / \mathrm{m}^{2}\right)$ & $26.9(4.8)$ & \\
\hline \multicolumn{3}{|l|}{ Smoking status } \\
\hline Never & & $30(35.3)$ \\
\hline Former & & $46(54.1)$ \\
\hline Current & & $9(10.6)$ \\
\hline \multicolumn{3}{|l|}{ Pack-years } \\
\hline $1-20$ & & $8(9.4)$ \\
\hline $21-40$ & & $10(11.8)$ \\
\hline $41-60$ & & $4(4.7)$ \\
\hline$\geq 61$ & & $7(8.2)$ \\
\hline \multicolumn{3}{|l|}{ Alcohol use } \\
\hline Never & & $28(32.9)$ \\
\hline Former & & $8(9.4)$ \\
\hline Current & & $49(57.6)$ \\
\hline \multicolumn{3}{|c|}{ Alcohol intake (units/day) } \\
\hline$<2$ & & $37(43.5)$ \\
\hline $2-4$ & & $12(14.1)$ \\
\hline$\geq 5$ & & 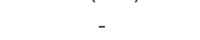 \\
\hline
\end{tabular}

\begin{tabular}{lc}
\hline Charlson comorbidity index & \\
Mild (0-3) & $4(4.7)$ \\
Moderate (4-5) & $10(11.8)$ \\
Severe ( $\geq 6)$ & $71(83.5)$ \\
\hline Weight loss in the past six months & \\
None & \\
$<10 \%$ & $56(65.9)$ \\
$\geq 10 \%$ & $23(27.1)$ \\
& $6(7.1)$ \\
\hline MUST-score & \\
$<2$ & $66(77.6)$ \\
$\geq 2$ & $19(22.4)$ \\
\hline TNM-stage & \\
I & $11(12.9)$ \\
II & $19(22.4)$ \\
III & $16(18.8)$ \\
IV & $39(45.9)$ \\
\hline Tumor type & \\
Primary & $65(76.5)$ \\
Second primary & $6(7.1)$ \\
Recurrent & $14(16.5)$ \\
\hline Tumor site & \\
Oral cavity & $51(60.0)$ \\
Nasopharynx & $2(2.4)$ \\
Oropharynx & $5(5.9)$ \\
Hypopharynx & $3(3.5)$ \\
Larynx & $8(9.4)$ \\
Skin & $11(12.9)$ \\
Salivary glands & $3(3.5)$ \\
Paranasal sinuses & $2(2.4)$ \\
\hline Treatment intention & \\
Curative & $71(83.5)$ \\
Palliative & $14(16.5)$ \\
\hline & \\
&
\end{tabular}

Results from the correlation analyses are shown in Table 2. Significant low to moderately strong correlation coefficients are seen for SMI and BMI $(r=0.49)$, SM and age $(r=-0.37)$, HGS and age $(r=-0.46)$, gait speed and age $(r=0.28)$ and for gait speed and HGS $(r=-0.39)$. 
Table 2. Pearson correlation analysis for variables associated with sarcopenia

\begin{tabular}{lccccc}
\hline Measures & SMI & HGS & Gait speed & Age & BMI \\
\hline SMI & - & 0.16 & -0.15 & $-0.37^{* *}$ & $0.49^{* *}$ \\
\hline HGS & 0.16 & - & $-0.39^{* *}$ & $-0.46^{* *}$ & -0.04 \\
\hline Gait speed & -0.15 & $-0.39^{* *}$ & - & $0.28^{*}$ & 0.05 \\
\hline Age & $-0.37^{* *}$ & $-0.46^{* *}$ & $0.28^{*}$ & - & -0.02 \\
\hline BMI & $0.49^{* *}$ & -0.04 & 0.05 & -0.02 & -
\end{tabular}

** Correlation is significant at the 0.01 level (2-tailed)

* Correlation is significant at the 0.05 level (2-tailed)

\section{Survival analysis}

Results from the Kaplan Meier survival analysis are shown in Figures 1-4. As seen in Figures 1-3, the median overall survival appears to be shorter for patients with low SMI compared with patients with high SMI, but this difference is not significant ( 11.24 versus 7.62 months; log rank test $p=0.40$ ), but this difference was not statistically significant. The differences in OS between patients with low HGS compared with patients with high HGS ( 7.52 versus 12.65 months; log rank test $\mathrm{p}=0.22$ ) and for patients with low gait speed compared with patients with high gait speed ( 7.89 versus 14.16 months; log rank test $p=0.22$ ) were not significant either. The median overall survival was significantly shorter for patients with sarcopenia compared to patients without sarcopenia (7.36 versus 13.22 months; log rank test $\mathrm{p}=0.02$ ), as is illustrated in Figure 4

Results from the univariate and multivariate cox regression analysis for overall survival are shown in Table 3. Sarcopenia (HR 2.28; $95 \% \mathrm{Cl}$ 1.12-4.65; $\mathrm{p}=0.02$ ) and TNM-stage ( $\mathrm{HR} 3.18 ; 95 \% \mathrm{Cl} 1.90-5.31 ; \mathrm{p}<0.01)$ were significant prognostic factors for overall survival in univariate cox regression analysis. In multivariate cox regression analysis; model 1 shows that sarcopenia remained a significant prognostic factor for overall survival independent of comorbidity, age and BMI. However, sarcopenia did not remain a significant prognostic factor when TNMstage was included (model 2). TNM-stage was a significant prognostic factor for overall survival in multivariate cox regression analysis, (HR 3.00; 95\% Cl 1.78-5.06; $\mathrm{p}<0.01)$

A subgroup analyses according to TNM-stage and treatment intention was performed, of which the results are shown in Table 4. Sarcopenia was a statistically significant prognostic factor for overall survival in patients with TNM-stage I-II and curative treatment intention ( $\mathrm{HR} 9.19 ; 95 \% \mathrm{Cl} 1.07-78.7 ; \mathrm{p}=0.04)$ and in all patients with a curative treatment intention (HR 2.80;95\% Cl 1.14-6.88; $\mathrm{p}=0.03$ ). However, sarcopenia was not a statistically significant prognostic factor for overal survival in patients with TNM-stage IV or palliative treatment intention (HR 1.16; $95 \% 0.53-2.57 ; \mathrm{p}=0.71$ ) and in all patients with a palliative treatment intention (HR $1.99 ; 95 \% \mathrm{Cl} 0.49-8.13 ; \mathrm{p}=0.34$ )

Figure 1. Kaplan-Meier overall survival curves and number at risk table for patients with low SMI and high SMI showed no statistically significant difference (Log rank ChiSquare $0.72 ; p=0.40$

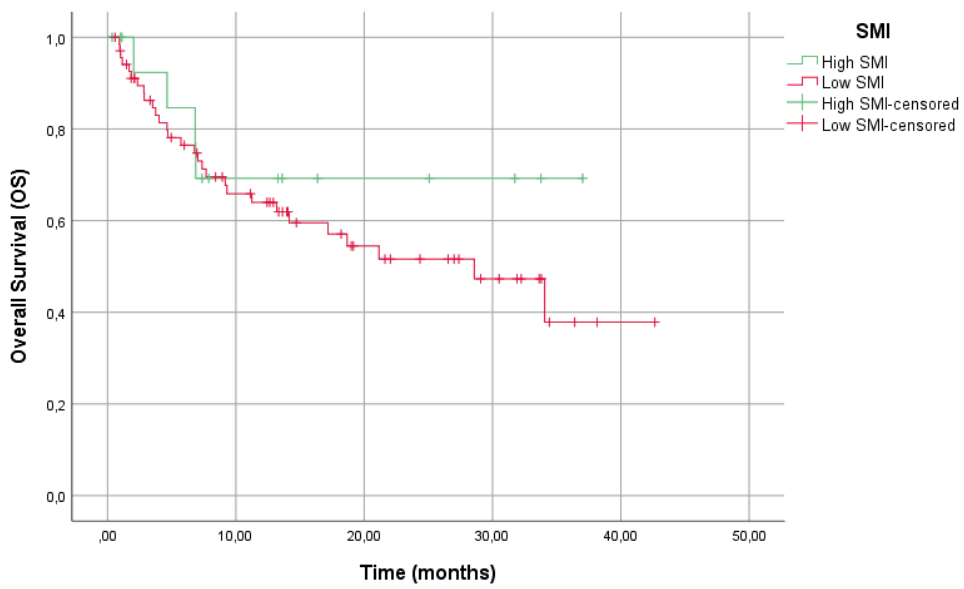

\begin{tabular}{lccccc}
\hline & $\mathrm{T}=\mathbf{0}$ & $\mathrm{T}=\mathbf{2 0}$ & $\mathrm{T}=\mathbf{4 0}$ & $\mathrm{T}=\mathbf{6 0}$ & $\mathrm{T}=\mathbf{8 0}$ \\
\hline Low SMI & 69 & 19 & 1 & - & - \\
High SMI & 16 & 4 & - & - & - \\
\hline
\end{tabular}


Figure 2. Kaplan-Meier overall survival curves and number at risk table for patients with low HGS and high HGS showed no statistically significant difference (Log rank ChiSquare 1.54; $p=0.21$ )



\begin{tabular}{lccccc}
\hline & $\mathrm{T}=\mathbf{0}$ & $\mathrm{T}=\mathbf{2 0}$ & $\mathrm{T}=\mathbf{4 0}$ & $\mathrm{T}=\mathbf{6 0}$ & $\mathrm{T}=\mathbf{8 0}$ \\
\hline Low HGS & 50 & 13 & 1 & - & - \\
High HGS & 35 & 10 & - & - & - \\
\hline
\end{tabular}

Figure 3. Kaplan-Meier overall survival curves and number at risk table for patients with low gait speed and high gait speed showed no statistically significant difference (Log rank Chi-Square 1.501; $p=0.22$ )

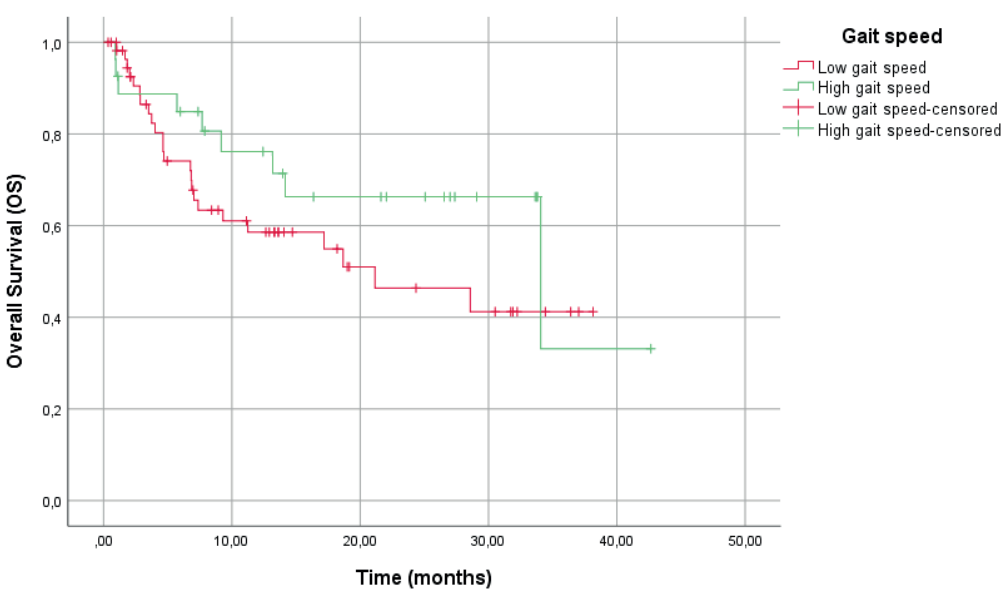

\begin{tabular}{lccccc}
\hline & $\mathrm{T}=\mathbf{0}$ & $\mathrm{T}=\mathbf{2 0}$ & $\mathrm{T}=\mathbf{4 0}$ & $\mathrm{T}=60$ & $\mathrm{~T}=\mathbf{8 0}$ \\
\hline Low gait speed & 58 & 11 & - & - & - \\
High gait speed & 27 & 12 & 1 & - & - \\
\hline
\end{tabular}


Figure 4. Kaplan-Meier overall survival curves and number at risk table for patients with and without sarcopenia showed statistically significant difference (Log rank Ch

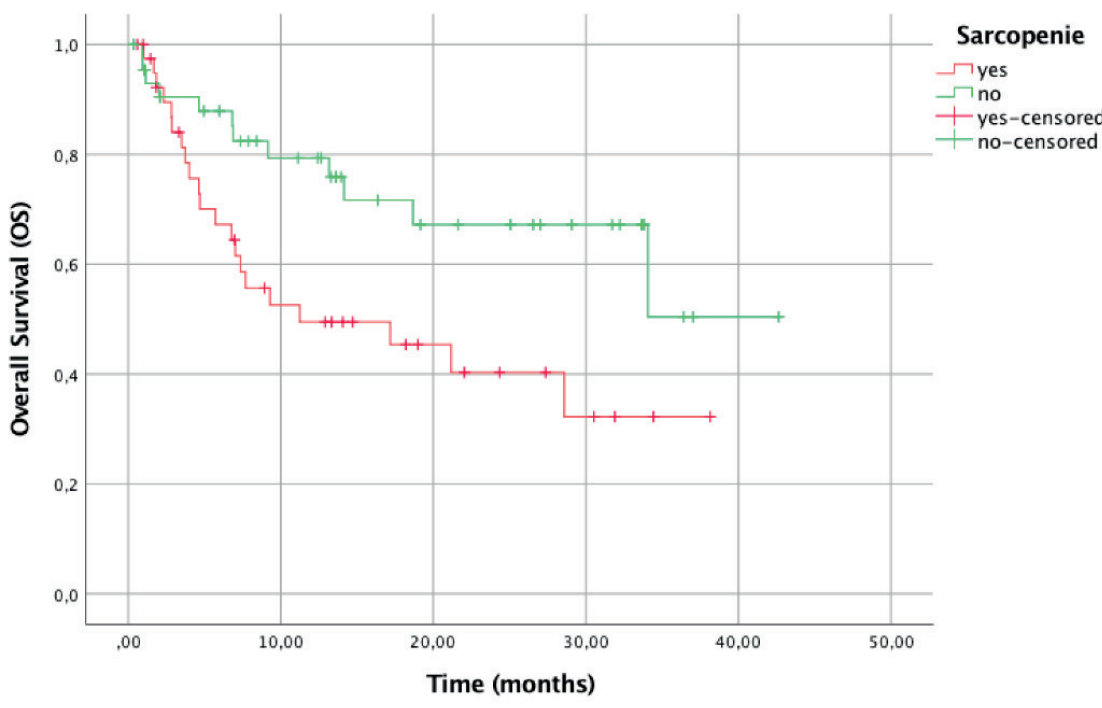

\begin{tabular}{lccccc}
\hline & $\mathrm{T}=\mathbf{0}$ & $\mathrm{T}=\mathbf{2 0}$ & $\mathbf{T}=\mathbf{4 0}$ & $\mathbf{T}=\mathbf{6 0}$ & $\mathbf{T}=\mathbf{8 0}$ \\
\hline Sarcopenia & 41 & 9 & 1 & - & - \\
Without sarcopenia & 44 & 14 & 1 & - & - \\
\hline
\end{tabular}

Table 3. Univariate and multivariate analysis of the hazard ratios for sarcopenia, age, $\mathrm{BMI}, \mathrm{CCl}$ and TNM stage as independent prognostic factors for overall survival.

\begin{tabular}{|c|c|c|c|c|c|c|c|c|c|}
\hline \multirow[t]{4}{*}{ Variable } & \multicolumn{9}{|c|}{ Overall survival } \\
\hline & \multicolumn{3}{|c|}{ Univariate analysis } & \multicolumn{6}{|c|}{ Multivariate analysis (*) } \\
\hline & \multirow[b]{2}{*}{ HR } & \multirow[b]{2}{*}{$95 \% \mathrm{Cl}$} & \multirow[b]{2}{*}{ P-value } & \multicolumn{3}{|c|}{ Model 1} & \multicolumn{3}{|c|}{ Model 2} \\
\hline & & & & HR & $95 \% \mathrm{Cl}$ & P-value & HR & $95 \% \mathrm{Cl}$ & P-value \\
\hline Sarcopenia & 2.28 & $1.12-4.65$ & $0.02^{*}$ & 2.32 & $1.06-5.07$ & $0.04^{*}$ & 1.86 & $0.85-4.01$ & 0.12 \\
\hline Age & 1.01 & $0.96-1.07$ & 0.72 & 1.0 & $0.95-1.06$ & 0.88 & 1.02 & $0.97-1.08$ & 0.45 \\
\hline \multicolumn{10}{|l|}{ BMI } \\
\hline$<18.5$ & 2.96 & $0.35-24.74$ & 0.32 & 3.69 & $0.43-37.38$ & 0.23 & 2.64 & $0.30-22.92$ & 0.38 \\
\hline $18.5-25$ & 2.14 & $0.84-5.43$ & 0.11 & 1.73 & $0.67-4.47$ & 0.26 & 0.97 & $0.35-2.68$ & 0.95 \\
\hline $25-30$ & 1.07 & $0.38-3.02$ & 0.90 & 0.79 & $0.27-2.31$ & 0.67 & 0.53 & $0.17-1.63$ & 0.27 \\
\hline$\geq 30$ & Ref. & & & Ref. & & & Ref. & & \\
\hline \multicolumn{10}{|l|}{$\mathrm{CCl}$} \\
\hline$<6$ & Ref. & & & Ref. & & & Ref. & & \\
\hline$\geq 6$ & 1.39 & $0.67-2.88$ & 0.37 & 1.16 & $0.54-2.53$ & 0.70 & 1.49 & $0.72-3.15$ & 0.29 \\
\hline TNM stage & 3.18 & $1.90-5.31$ & $<0.01^{* *}$ & - & - & - & 3.00 & $1.78-5.06$ & $<0.01^{* *}$ \\
\hline
\end{tabular}

** Correlation is significant at the 0.01 level (2-tailed)

Correlation is significant at the 0.05 level (2-tailed)

(*) Model 1 includes the variables; sarcopenia, age, BMI and CCI. Model 2 includes all the selected variables.

Table 4. Subgroup analyses according to TNM-stage, treatment intention and sarcopenia showed sarcopenia as a statistically significant prognostic factor for overall survival in patients with TNM-stage I-III with a curative treatment intention (HR 9.19; 95\% $\mathrm{Cl}$ 1.07-78.74; $p=0.04$ ) and in all patients with curative treatment intention (HR 2.80 $95 \% \mathrm{Cl} 1.14-6.88 ; \mathrm{p}=0.03$ )

\begin{tabular}{lcccc}
\hline \multirow{2}{*}{ Subgroup } & \multicolumn{4}{c}{ Overall survival } \\
\cline { 2 - 5 } & \multicolumn{4}{c}{ Sarcopenia } \\
\cline { 2 - 5 } & Frequency & HR & $95 \% \mathrm{Cl}$ & P-value \\
\hline TNM-stage I-III and curative treatment intention & 41 & 9.19 & $1.07-78.7$ & $0.04^{*}$ \\
\hline TNM-stage IV or palliative treatment intention & 44 & 1.16 & $0.53-2.57$ & 0.71 \\
\hline Curative treatment intention & 71 & 2.80 & $1.14-6.88$ & $0.03^{*}$ \\
\hline Palliative treatment intention & 14 & 1.99 & $0.49-8.13$ & 0.34 \\
\hline * Correlation
\end{tabular}

Correlation is significant at the 0.05 level (2-tailed) 


\section{Discussion}

Sarcopenia is a common and highly prevalent clinical problem in the elderly patient. Literature showed that sarcopenia is associated with several negative outcomes, however literature mainly focuses on radiologically assessed low SMM rather than the combination of SMM and $\mathrm{MF}^{5 .-15}$ In addition, no studies report on the impact of sarcopenia on survival in the elderly head and neck cancer patient. Identification of the impact of low SMM and low MF on prognosis in the elderly head and neck cancer patient will stimulate the development of novel interventions to gain SMM and MF which may improve the prognosis of these patients. Regardless of the success of an intervention, information on prognosis can be used for patient counseling and treatment decision making.

In this study, we included 85 patients of whom 41 patients (48.2\%) were classified as sarcopenic. This number is in accordance with recent medical literature which estimated the prevalence of sarcopenia in elderly patients diagnosed with different types of cancer between 14\%-78.7\%. ${ }^{21}$ The prevalence estimates of sarcopenia in the elderly non-cancer patients are lower, ranging between $5-50 \%$. Sarcopenia is prevailing in elderly cancer patients because of the frequent weight loss caused by low food intake, increased catabolic pathways, increased inflammation, increased lipolysis and increased proteolysis associated with both old age and malignancy. ${ }^{21}$

This study shows that SMM, muscle strength and physical functioning separately had no significant prognostic value for overall survival. A combination of muscle mass and muscle strength or muscle performance did show a significant prognostic value for overall survival in elderly patients with head and neck cancer. This is in accordance with previous studies in other tumor types, which have demonstrated that not only SMM but also MF is related with several health outcomes. ${ }^{21-23}$ Previous studies in patients with esophageal cancer did not show a significant prognostic value of sarcopenia on overall survival, however sarcopenia was defined as low radiologically assessed SMM only rather than a combination of low SMM and low MF.24-27 Our study highlights the importance of defining sarcopenia as a combination of SMM and MF.

In multivariate analysis including the covariates age, BMI and comorbidity; sarcopenia remained a statistically significant prognostic factor for overall survival. When including TNM-stage in the multivariate analysis, sarcopenia did not remain a statistically significant prognostic factor for overall survival.
Subgroup analyses according to TNM-stage and, treatment intention shows that sarcopenia is a statistically significant prognostic factor for overall survival in patients with TNM-stage I-III and in patients with curative treatment intention. In patients with TNM-stage IV or in patients with palliative treatment intention, sarcopenia is not a statistically significant prognostic factor for overall survival. In this study, 39 patients (45.9\%) had an TNM-stage IV and 14 patients (16.5\%) had a palliative treatment intention. It is possible that sarcopenia did not remain a significant prognostic factor in model 2 of the multivariate analysis because of the high number of patients with TNM-stage IV and palliative treatment intention. This finding is in accordance with a previous study performed in patients with gastric cancer which showed that sarcopenia is a significant prognostic factor for overall survival in patients with TNM-stage $\mathrm{II}-\mathrm{III}{ }^{28}$ It is also in accordance with a recent systematic review, which showed that sarcopenia is a significant prognostic factor for overall survival in different types of cancers independent of TNM-stage. ${ }^{29}$

The existing literature on sarcopenia in patients with head and neck cancer is scarce and focuses mainly on low SMM in patients who receive (chemo)radiotherapy ${ }^{6}$ or patients who undergo a total laryngectomy..$^{910}$ To our knowledge, our study is the first to investigate the impact of sarcopenia, defined as a combination of SMM and MF, in elderly (70-year old) head and neck cancer patients.

This study has some limitations. It was designed as a retrospective singlecenter study, which increases the risk for systemic errors. It had limited number of included patients which may have led to type II errors. Only patients with available data on SMM and MF were included in the study. As it is more likely that MF parameters were examined for frail patients than for fit patients, this may have resulted in a biased study population in which it is probably more difficult to show the prognostic value of sarcopenia. Therefore, sarcopenia as combination of SMM and MF should be further evaluated as a prognostic factor for overall survival in elderly patients with head and neck cancer. In retrospective studies, data on MF will probably rarely be available, whereas $\mathrm{CT}$ or MRI is often routinely performed in head and neck cancer patients. We propose to conduct further prospective studies for the measurement of both MF and SMM and to perform routine handgrip strength measurements in every newly diagnosed head and neck cancer patient. Concerning the imaging techniques used to assess SMM, we decided to include both CT scans and MRI scans of the head and neck area to assess SMM in order to maximize the number of patients that could be included. Whenever available, we used CT imaging instead of MRI because most research on SMM in cancer patients is performed using CT imaging. However, the CT measurement 
method for SMM was formulated on MRI-based research. ${ }^{30,31}$ Theoretically there is no difference in SMM between CT imaging and MRI, as both methods are very accurate for SMM assessment. Therefore, we believe it is acceptable to use MRI for SMM measurement when CT imaging is not available. Research should be conducted to investigate this further.

In conclusion, sarcopenia is present in half of the elderly HNSCC patients. Skeletal muscle mass index and muscle function, as determined by muscle strength or physical performance measurements, were not prognostic separately in elderly HNSCC patients, but the combination of both was prognostic for overall survival. Therefore, it may be preferable to define sarcopenia as the combination of low skeletal muscle mass and low muscle function and not by radiologically assessed skeletal muscle mass alone.

\section{References}

. Cruz-Jentoft AJ, Baeyens JP, Bauer JM, Boirie Y, Cederholm T, Landi F, Martin FC, Michel JP, Rolland Y, Schneider SM, Topinkova E, Vandewoude M, Zamboni M. Sarcopenia: European consensus on definition and diagnosis: Report of the European Working Group on Sarcopenia in Older People. Age and ageing. 2010;39(4):412-23.

2. von Haehling S, Morley JE, Anker SD. An overview of sarcopenia: facts and numbers on prevalence and clinical impact. Journal of cachexia, sarcopenia and muscle. 2010;1 (2):129-33,

3. Goodpaster BH, Park SW, Harris TB, Kritchevsky SB, Nevitt M, Schwartz AV, Simonsick EM, Tylavsky FA, Visser M, Newman AB. The loss of skeletal muscle strength, mass, and quality in older adults: the health, aging and body composition study. J Gerontol A Biol Sci Med Sci. 2006 Oct;61(10):1059-64.

4. Janssen I, Baumgartner RN, Ross R, Rosenberg IH, Roubenoff R. Skeletal muscle cutpoints associated with elevated physical disability risk in older men and women. American journal of epidemiology. 2004;159(4):413-21.

5. Shachar SS, Williams GR, Muss HB, Nishijima TF. Prognostic value of sarcopenia in adults with solid tumours: A meta-analysis and systematic review. Eur J Cancer. 2016 Apr;57:58-67.

6. Wendrich AW, Swartz JE, Bril SI, Wegner I, de Graeff A, Smid EJ, de Bree R, Pothen AJ. Low skeletal muscle mass is a predictive factor for chemotherapy dose-limiting toxicity in patients with locally advanced head and neck cancer. Oral Oncol. 2017 Aug; 71:26-33.

7. Prado CM, Baracos VE, McCargar LJ, Reiman T, Mourtzakis M, Tonkin K, Mackey JR, Koski S, Pituskin E, Sawyer MB. Sarcopenia as a determinant of chemotherapy toxicity and time to tumor progression in metastastic breast cancer patients receiving capecitabine treatment. Clin Cancer Res. 2009 Apr 15;15(8):2920-6.

8. Antoun S, Baracos VE, Birdsell, Escudier B, Sawyer MB. Low body mass index and sarcopenia associated with dose-limiting toxicity of sorafenib in patients with renal cell carcinoma. Ann Oncol. 2010; 21:1594-1598.

9. Achim V, Bash J, Mowery A, Guimaraes AR, Li R, Schindler J, Wax M, Andersen P, Clayburgh D. Prognostic Indication of Sarcopenia for Wound Complication After Total Laryngectomy. JAMA Otolaryngol Head Neck Surg. 2017 Dec 1;143(12):1159-1165.

10. Bril SI, Pezier TF, Tijink BM, Janssen LM, Braunius WW, de Bree R (2018) Preoperative low skeleta muscle mass as a risk factor for pharyngocutaneous fistula and decreased overall survival in patients undergoing total laryngectomy. Head Neck. 2019 Jun;41(6):1745-1755.

11. Ida S, Watanabe M, Yoshida N, Baba Y, Umezaki N, Harada K, Karashima R, Imamura Y, Iwagam S, Baba H. Sarcopenia is a predictor of postoperative respiratory complications in patients with esophageal cancer. Ann Surg Oncol. 2015; 22(13):4432-7.

12. van Vugt IL, Braam HJ, van Oudheusden TR, Vestering A, Bollen TL, Wiezer MJ, de Hingh IH, van Ramshorst B, Boerma D. Skeletal muscle depletion is associated with severe postoperative complications in patients undergoing cytoreductive surgery with hyperthermic intraperitotive chemothera for peris

13. Nishikawa D, Hanai N, Suzuki H, Koide Y, Beppu S, Hasegawa Y. The Impact of Skeletal Muscle Depletion on Head and Neck Squamous Cell Carcinoma. ORL J Otorhinolaryngol Relat Spec. 2018; 80:1-9.

14. Miyamoto Y, Baba Y, Sakamoto Y, Ohuchi M, Tokunaga R, Kurashige J, Hiyoshi Y, Iwagami S, Yoshida N, Yoshida M, Watanabe $\mathrm{M}, \mathrm{Baba}$. Sarcopenia is a negative prognostic factor after curative resection of colorectal cancer. Ann Surg Oncol. 2015; 22:2663-2668.

15. Okumura S, Kaido T, Hamaguchi Y, Fujimoto Y, Masui T, Mizumoto M, Hammad A, Mori A, Takaori $\mathrm{K}$, Uemoto $\mathrm{S}$. Impact of preoperative quality as well as quantity of skeletal muscle on survival after resection of pancreatic cancer. Surgery. 2015; 157(6):1088-98.

16. Fukuda $Y$, Yamamoto $K$, Hirao $M$ et al. Sarcopenia is associated with severe postoperative complications in elderly gastric patients undergoing gastrectomy. Gastric Cancer. 2016 19(3):986-93. 
17. Heymsfield SB, Wang Z, Baumgartner RN, Ross R. Human body composition: advances in models and methods. Annu Rev Nutr. 1997; 17:527-58.

18. Swartz JE, Pothen AJ, Wegner I, Smid EJ, Swart KM, de Bree R, Leenen LP, Grolman W.. Feasibility of using head and neck CT imaging to assess skeletal muscle mass in head and neck cancer 62:28-33.

19. Lauretani F, Russo CR, Bandinelli S, Cavazzini C, Di lorio A, Corsi AM, Rantanen T, Guralnik $J M$, Ferrucci L. Age-associated changes in skeletal muscles and their effect on mobility: an pertion physiology. 2003; 95(5):1851-60.

20. Studenski S, Perera S, Wallace D, Chandler JM, Duncan PW, Rooney E, Fox M, Guralnik JM. Physical performance measures in the clinical setting. J Am Geriatr Soc. 2003; 51(3): 314-22.

Phys Med Rehabil 2016, 4:3.

N.N. Hairi, R.G. Cumming, V. Naganathan, Handelsman DJ, Le Couteur DG, Creasey H, Waite LM, Seibel MJ, Sambrook PN. Loss of muscle strength, mass (sarcopenia), and quality (specific force) and its relationship with functional limitation and physical disability. The Concord Health and

23. E. Zoico, V. Di Francesco, J. Guralnik, Mazzali G, Bortolani A, Guariento S, Sergi G, Bosello O, Zamboni M. Physical disability and muscular strength in relation to obesity and different body composition indexes in a sample of healthy elderly women. Int J Obes Relat Metab Disord. 2004; 28(2): 234-41

24. Grotenhuis BA, Shapiro J, van Adrichem S, de Vries M, Koek M, Wijnhoven BPL, van Lanscho J. Sarcopenia/muscle mass is not a prognostic factor for short- and long-term outcome after esophagectomy for cancer. World J Surg. 2016;40(11):2698-2704.

25. Awad S, Tan BH, Cui H, Bhalla A, Fearon KC, Pearsons SL, Catton JA, Lobo DN. Marked changes in body composition following neoadjuvant chemotherapy for oesophagogastric cancer. Clin Nutr. 2012; 31:74-77.

26. Yip C, Goh V, Davies A, Gossage J, Mitchell-Hay R, Hynes O, Maisey N, Ross P, Gaya A, Landau DB, Cook GJ, Griffin N, Mason R. Assessment of sarcopenia and changes in body composition after neoadjuvant chemotherapy and associations with clinical outcomes in oesophageal cancer. Eur Radiol. 2014; 24:998-1005.

27. Sheetz KH, Zhao L, Holcombe SA, Wang SC, Reddt RM, Lin J, Orringer MB, Chang AC. Decreased core muscle size is associated with worse patient survival following esophagectomy for cancer. Dis Esophagus. 2013; 26:716-722.

28. F. Pamoukdjian, T. Bouillet, V. Levy, M. Soussan, L. Zelek, E. Paillaud. Prevalence and predictive value of pre-therapeutic sarcopenia in cancer patients: A systematic review. Clinical Nutrition; 2018; 37(4):1101-1113. DOI: 10.1016/j.clnu.2017.07.010

29. Zhuang CL, Huang DD, Pang WY, Zhou CJ, Wang SL, Lou N, Ma LL, Yu Z, Shen X. Sarcopenia is an Independent Predictor of Severe Postoperative Complications and Long-Term Survival After Radical Gastrectomy for Gastric Cancer: Analysis from a Large-Scale Cohort. Medicine. 2016 ;95(13): e3164.

30. Prado CM, Lieffers JR, McCargar LJ, Reiman T, Sawyer MB, Martin L, et al. Prevalence and clinical implications of sarcopenic obesity in patients with solid tumours of the respiratory and gastrointestinal tracts: a population-based study. Lancet Oncol. 2008;9(7):629-35.

31. Shen W, Punyanitya M, Wang Z, Gallagher D, St-Onge MP, Albu J, et al. Total body skeleta muscle and adipose tissue volumes: estimation from a single abdominal cross-sectional image. J Appl Physiol (1985). 2004:97(6):2333-8. 


\section{Chapter 8}

Skeletal muscle mass is an imaging biomarker for decreased survival in patients with oropharyngeal squamous cell carcinoma

Najiba Chargi, Sandra I. Bril, Justin E. Swartz, Inge Wegner, Stefan M. Willems, Remco de Bree 


\section{Abstract}

Objectives Low skeletal muscle mass (SMM) and sarcopenic obesity (co-presence of low SMM and obesity) are emerging prognosticators in oncology, but the prevalence and prognostic value in oropharyngeal squamous cell carcinoma (OPSCC) is not yet known.

Methods Patients with OPSCC, curative treatment intention and pre-treatment diagnostic imaging of the head and neck area were included. Patients with unknown HPV-status, palliative treatment intention or unavailable imaging were excluded. Relevant demographic and clinical characteristics were collected between 2009-2016. Patients were stratified into a low-, intermediate-, and highrisk group according to HPV-status, amount of pack-years, tumor and nodal stage. SMM was radiologically measured and cutoff values were determined by optimal stratification. The prognostic value of low SMM and sarcopenic obesity for overal survival (OS) and disease-free survival (DFS) was determined by Cox regression analysis and Kaplan Meier survival curves.

Results In 216 patients, low SMM and sarcopenic obesity were present in 140 (64.8\%) and 13 (6.0\%) patients, respectively. On multivariate analysis, stratification into a high-risk group (HPV-negative status with $\geq 10$-pack-years or T4-stage) was a prognostic factor for OS and DFS (HR 2.93, p<0.01) (HR 4.66, p<0.01). Of specific interest, sarcopenic obesity was a strong negative prognostic factor for OS and DFS (HR 4.42, p<0.01) and (HR 3.90, p<0.05), independent from other well-known prognostic factors such as HPV-status.

Conclusion Low skeletal muscle mass is highly prevalent in OPSCC patients. Sarcopenic obesity is a novel pretreatment prognosticator for OS and DFS in OPSCC and should therefore be considered in clinical decision making.

\section{Introduction}

Head and neck cancers (HNCs) are among the most frequent tumors in the world with an estimated 835.000 new cases and 428.000 deaths in $2018 .^{1}$ The vast majority of HNCs are head and neck squamous cell carcinomas (HNSCCs), and most are related to alcohol consumption and/or smoking. ${ }^{23}$ There has been a rise in the incidence of HNSCC over the past decade, in particular, the incidence of oropharyngeal squamous cell carcinoma (OPSCC). This is largely due to a specific increase in incidence of a particular subset of OPSCC, which is driven by high risk Human Papilloma Virus (HPV) infection. ${ }^{4}{ }^{5}$ In general, HPV-positive OPSCC has a better prognosis than HPV-negative OPSCC. ${ }^{6}$ Other known prognostic factors in OPSCC include age, tumor stage, nodal stage, and comorbidities. 78 For HPV-related OPSCC, several risk models have been published in medical literature in order to gain more precise prognostic information for this specific subset of HNSCCs patients, which may allow for development of treatment deintensification approaches for HPV-associated OPSCC. ${ }^{9-12}$ Ang et al. were the first to propose a risk stratification model for OPSCC, which stratified patients according to HPV-status, smoking status, tumor and nodal stage into a low, intermediate and high risk of death. ${ }^{9}$ Although previously reported risk models included a variety of known prognostic factors in OPSCC, none included body composition as a possible interacting variable.

Over the last decade, the radiological assessment of individual body composition has increasingly gained interest in oncological patients. ${ }^{13}$ Sarcopenia, sometimes also termed low skeletal muscle mass (SMM) or low lean body mass, is traditionally described as a geriatric syndrome consisting of both the specific loss of SMM and the decrease of skeletal muscle function. ${ }^{14}$ Sarcopenia is a multifactorial syndrome risk factors include malnutrition, immobility and illness. ${ }^{14}$ In oncological studies, sarcopenia is often defined as low SMM only, because skeletal muscle function tests are not commonly performed in routine clinical practice. Sarcopenic obesity is described as the co-presence of both low SMM and obesity. ${ }^{15} 16$

In several retrospective studies, low SMM and sarcopenic obesity have been associated with increased rates of postoperative complications, chemotherapyrelated toxicity, and decreased survival rates in colon cancer, breast cancer, lung cancer, and pancreatic cancer, amongst others. ${ }^{17} 1819$ Research on the prevalence, predictive value and prognostic impact of low SMM and sarcopenic obesity in HNC patients has more recently been initiated. In patients with locally advanced HNSCC undergoing chemoradiotherapy, sarcopenia was associated with a trifold risk of 
chemotherapy dose-limiting toxicity. ${ }^{20}$ Pre-treatment low SMM was associated with an increased incidence of pharyngocutaneous fistula and decreased overall survival in HNSCC patients undergoing a total laryngectomy. ${ }^{21} 22$

A recent study in OPSCC patients showed an association between low SMM and decreased overall survival, independent of HPV-status. ${ }^{23}$ In this study, only patients with advanced OPSCC were included and SMM was assessed on a pre-treatment PET-CT scan of the abdomen. Even though measurement of SMM on a CT scan of the abdomen at the level of the third lumbar vertebra (L3) is very common in oncological research ${ }^{17}$, this may lead to an inclusion bias in HNC patients because not all patients will undergo a PET-CT during the diagnostic work-up. ${ }^{24}$ Recently, a novel SMM assessment method at the level of the third cervical vertebra (C3) was published. ${ }^{25}{ }^{26}$ Imaging at the level of $\mathrm{C} 3$ is almost always available in HNC patients because diagnostic imaging of the head and the neck area is used for staging, which allows the routine assessment of SMM.

In this study, we aim to investigate the prevalence and prognostic value of pretreatment low SMM and sarcopenic obesity as measured at the level of C3 in a large cohort of OPSCC patients corrected for known prognostic factors including age, weight loss 6 months prior to diagnosis, comorbidities, HPV-status, and TNM-stage.

\section{Material and methods}

\section{Ethical approva}

The design of this study was approved by the Medical Ethical Research Committee of the University Medical Center Utrecht (approval ID 17-365/C). All procedures in this study were in accordance with the ethical standards of the institutional and/or national research committee and with the 1964 Helsinki declaration and its later amendments or comparable ethical standards. All data were handled according to general data protection regulation (GDPR)

\section{Patients and study design}

A retrospective cohort study was conducted in 241 OPSCC patients diagnosed and treated with curative intent at the University Medical Center Utrecht, Utrecht, The Netherlands, between 2009 and 2016. Patients with OPSCC were included if they had recent ( $\leq 1$ months prior) pre-treatment imaging scans (CT or MRI) of the head and neck at the level of the third cervical vertebrae (C3). The median time between imaging and treatment was 0.79 month (IQR 0.56-1.12). Patients were excluded if they had a palliative treatment intent $(n=17)$ or if diagnostic imaging was of poor quality which impaired measurements of SMM $(n=8)$. In total, 216 OPSCC patients were included.

Relevant demographic and clinical variables were collected from patients' medica record. Demographic variables included; sex and age at diagnosis. Clinica variables included; length and weight at diagnosis, body-mass-index (BMI) percentage of weight loss in the six months prior to diagnosis, smoking status, amount of pack-years, alcohol intake, comorbidities as expressed by the Adult Comorbidity Evaluation-27 (ACE-27) score, tumor localization, date of histologic diagnosis, HPV-status, tumor staging according to the tumor-node-metastasis (TNM) 7th Edition IUCC manual, treatment modality (chemotherapy, radiotherapy, surgery or a combination) and survival data.

HPV-status was determined using the algorithm described by Smeets et al: a p16 staining was performed. In case of a positive result this was followed by a PCR on HPV. ${ }^{27}$ Patients with known HPV-status were stratified into risk groups as described by Ang et al: HPV-positive patients with less than 10 pack-years or with more than 10-pack-years but a NO-N2a nodal stage were considered to be at low risk, whereas HPV-positive patients with more than 10 pack-years and a N2b-N3 nodal stage and HPV-negative patients with less than 10 pack-years and a T1-3 tumor stage were considered to be at a intermediate risk, HPV-negative patients with more than 10 pack-years or with less than 10 pack-years but a T4 tumor stage were considered to be at high risk. ${ }^{9}$

Overall survival (OS) was defined as the time between the date of histologic diagnosis and death, or date of last follow-up. Disease-free survival (DFS) was defined as the time between the date of histologic diagnosis and the date of pathologic confirmed recurrence or date of last follow-up, whichever occurred first. Whenever possible, 5-year OS and DFS rates were calculated.

\section{Body composition measurement}

SMM was measured as muscle cross-sectional muscle area (CSA) on pre-treatment CT or MRI imaging of the head and neck area at the level of the third cervica vertebrae (C3). The axial slide of the imaging which showed both transverse processes and the entire vertebral arc was selected for segmentation of muscle tissue. For CT imaging, muscle area was defined as the pixel area between the radiodensity range of -29 and +150 Hounsfield Units (HU), which is specific for 
muscle tissue. ${ }^{28}$ For MRI, muscle area was manually segmented, and fatty tissue was manually excluded. The overall intraclass correlation coefficient for the muscle CSA obtained by CT and MRI is excellent $(0.97, \mathrm{p}<0.01)$ (unpublished data).

The CSA was calculated as the sum of the delineated areas of the paravertebral muscles and both sternocleidomastoideus muscles. An example of segmentation at the level of $\mathrm{C} 3$ is shown in Figure 1.

\section{Figure 1. Example of segmentation of skeletal muscle tissue at the level of the third} cervical vertebra (C3)

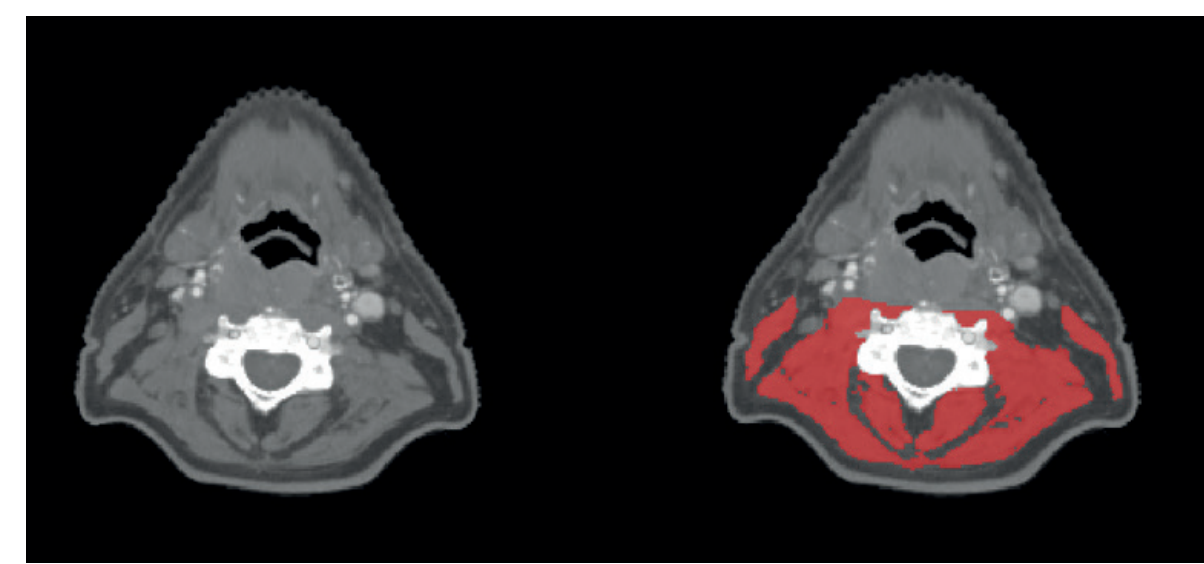

Figure shows two identical axial CT-slides at the level of C3; left shows the muscle tissue unsegmented, right shows both sternocleidomastoideus and paravertebral muscles segmented in red.

Segmentation of muscle tissue was manually performed using the commercially available software package SliceOmatic (Tomovision, Canada) by a single researcher (N.C). CSA at the level of C3 was converted to CSA at the level of L3 using a previously published formula, as shown in formula $1 .{ }^{25}$ The lumbar skeletal muscle index (LSMI) was calculated by correcting SMM at the level of L3 for squared height, as shown in formula 2. Sarcopenic obesity was defined as the combination of low SMM in combination with a $\mathrm{BMI} \geq 27 \mathrm{~kg} / \mathrm{m}^{2}$.

Formula 1: CSMA at L3 $\left(\mathrm{cm}^{2}\right)=27.304+1.363 * \mathrm{CSMA}$ at C3 $\left(\mathrm{cm}^{2}\right)+0.640 *$ Weight $(\mathrm{kg})+26.442 *$ Gender (Gender $=1$ for female, 2 for male) $-0.671 *$ Age (years)
Formula 2: Lumbar SMI $\left(\mathrm{cm}^{2} / \mathrm{m}^{2}\right)=\mathrm{CMSA}$ at L3 $/\left(\right.$ height $\left.^{2}\right)$

\section{Statistical methods}

The optimal stratification method was used to determine cohort specific cutoff values of the SMM. This method is the preferred method in literature and is based on log rank statistics to find the most significant cutoff value for SMM with respect to overall and disease-free survival. ${ }^{29}$ End-points (OS and DFS) specific cutoff values were determined for the lumbar SMM index and these were used to categorize patients into patients with low SMM and without low SMM for each endpoint.

Data analysis was performed using IBM SPSS statistics 25. Descriptive statistics for continuous variables with a normal distribution were presented as mean with standard deviation (SD). Normality was investigated using the KolmogorovSmirnov test. The variables age at diagnosis and units of alcohol intake per day were not normally distributed. Variables with a skewed distribution were presented as median with interquartile range (IQR). Categorical variables were presented as frequencies and percentages. Chi-square statistics were used for analyzing differences between the frequencies of each categorical variable with the presence or absence of low SMM. Independent sample student's $t$-tests were used for comparing the means of the normally distributed continuous variables with the presence or absence of low SMM. Statistical significance was evaluated at the 0.05 level using 2 -tailed tests.

Survival was visualized using Kaplan Meier survival curves and number at risk tables. Survival analysis was performed for the subset of patients with known HPV status, patients with missing HPV-status $(n=42)$ were excluded. A Cox proportiona hazard regression model was used for univariate and multivariate analysis of overall and disease-free survival. Covariates used in the univariate analysis were selected based on clinical relevance based on literature. Covariates used in the multivariate analysis were selected based on statistical significance $(p<0.05)$ in univariate cox regression analysis. In multivariate Cox regression analysis, two models were constructed, each examining the role of low SMM and sarcopenic obesity separately. Statistical significance was evaluated the 0.05 level using 2-tailed tests. 


\section{Results}

A total of 216 OPSCC patients with curative treatment intent and adequate pretreatment imaging of the head and neck area at the level of $\mathrm{C} 3$ were included Of these patients, 174 patients were identified with known HPV-status. Patient, disease and outcome characteristics are shown in Table 1.

Table 1. Demographic and clinical characteristics of patients with and without low SMM

\begin{tabular}{|c|c|c|c|c|}
\hline & Low SMM & Without low SMM & & \\
\hline Variables & $\mathrm{N}=140$ & $\mathrm{~N}=76$ & $x^{2}$ & p-value \\
\hline HPV-status $(\mathbf{n}, \%)$ & 236 & 36 & 1288 & ก $007 * *$ \\
\hline
\end{tabular}

\begin{tabular}{lcccc} 
Positive & 33 & 23.6 & 36 & 47.4 \\
Negative & 77 & 55 & 28 & 36.8 \\
Unknown & 30 & 21.4 & 12 & 15.8 \\
\hline
\end{tabular}

\begin{tabular}{llllll} 
Unknown & 30 & 21.4 & 12 & 15.8 & \\
\hline HPV risk group $(\mathbf{n}, \%)$ & & & & & $\mathbf{0 . 0 0 1 * *}$
\end{tabular}

$\begin{array}{llllll}\text { Low } & 23 & 13.2 & 28 & 16.1 & 11.68\end{array}$

Intermediate

$\begin{array}{llll}19 & 10.9 & 12 & 6.9 \\ 68 & 39.1 & 24 & 13.8\end{array}$

High

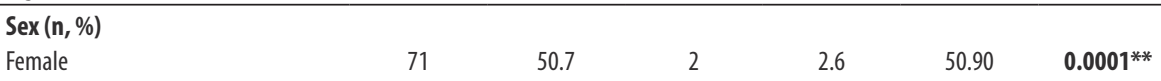

\begin{tabular}{|c|c|c|c|c|c|c|}
\hline Female & 71 & 50.7 & 2 & 2.6 & 50.90 & $0.0001^{* *}$ \\
\hline Male & 69 & 49.3 & 74 & 97.4 & & \\
\hline Age (years) (M, SD) & 63.6 & 9.6 & 60.3 & 9.7 & NA & $0.02^{*}$ \\
\hline
\end{tabular}

\section{$\operatorname{BMI}\left(\mathbf{k g} / \mathbf{m}^{2}\right)(\mathbf{n}, \%)$}

$\begin{array}{lc}\text { BMI }\left(\mathbf{k g} / \mathbf{m}^{2}\right)(\mathbf{n}, \%) & 40 \\ <20 & 76 \\ 20-24.9 & 19 \\ 25-29.9 & 5\end{array}$

\section{Weight loss 6 months prior to} diagnosis $(n, \%)$

Non

$<10 \%$

$\geq 10 \%$

\begin{tabular}{lcccccc}
\hline Smoker $(\mathbf{n}, \%)$ & & & & & & \\
No & 13 & 9.3 & 15 & 19.7 & 4.86 & 0.09 \\
Former & 45 & 32.1 & 23 & 30.3 & & \\
Current & 82 & 58.6 & 38 & 50 & & \\
\hline Pack-years (n, \%) & & & & & & \\
0 & 13 & 9.4 & 15 & 20.8 & 10.11 & $\mathbf{0 . 0 4}$ \\
$1-15$ & 25 & 18 & 13 & 18.1 & & \\
$16-25$ & 15 & 10.8 & 11 & 15.3 & & \\
$26-40$ & 30 & 21.6 & 17 & 23.6 & & \\
$\geq 41$ & 56 & 40.3 & 16 & 22.2 & & \\
\hline Alcohol use $(\mathbf{n}, \%)$ & & & & & & \\
No & 19 & 13.6 & 13 & 17.1 & 2.13 & 0.36 \\
Yes & 100 & 71.4 & 47 & 61.8 & & \\
Former & 21 & 15 & 16 & 21.1 & & \\
\hline
\end{tabular}

\begin{tabular}{|c|c|c|c|c|c|c|}
\hline $\begin{array}{l}\text { Alcohol units/day } \\
\text { (M, SD) }\end{array}$ & 3.3 & 2.7 & 4.4 & 3.9 & NA & 0.11 \\
\hline \multicolumn{7}{|l|}{ ACE-27 score $(n, \%)$} \\
\hline Non & 38 & 27.1 & 19 & 25 & 1.35 & 0.72 \\
\hline Mild & 34 & 24.3 & 19 & 25 & & \\
\hline Moderate & 41 & 29.3 & 27 & 35.5 & & \\
\hline Severe & 27 & 19.3 & 11 & 14.5 & & \\
\hline \multicolumn{7}{|l|}{ Localization $(\mathbf{n}, \%)$} \\
\hline Tonsil & 33 & 23.6 & 24 & 31.6 & 2.02 & 0.57 \\
\hline Base of tongue & 6 & 11.4 & 6 & 7.9 & & \\
\hline Soft palate & 7 & 5 & 3 & 3.9 & & \\
\hline Oropharynx n.o.s & 84 & 60 & 43 & 56.6 & & \\
\hline \multicolumn{7}{|l|}{ Tumor stage $(n, \%)$} \\
\hline $\mathrm{T} 1$ & 30 & 21.4 & 16 & 21.1 & 2.03 & 0.74 \\
\hline T2 & 52 & 37.1 & 31 & 40.8 & & \\
\hline $\mathrm{T} 3$ & 26 & 18.6 & 15 & 19.7 & & \\
\hline T4a & 25 & 17.9 & 13 & 17.1 & & \\
\hline $\mathrm{T} 4 \mathrm{~b}$ & 7 & 5 & 1 & 1.3 & & \\
\hline \multicolumn{7}{|l|}{ Nodal stage $(n, \%)$} \\
\hline No & 66 & 47.1 & 24 & 31.6 & 9.73 & 0.08 \\
\hline $\mathrm{N} 1$ & 23 & 16.4 & 15 & 19.7 & & \\
\hline N2a & 4 & 2.9 & 6 & 7.9 & & \\
\hline $\mathrm{N} 2 \mathrm{~b}$ & 28 & 20 & 23 & 30.3 & & \\
\hline $\mathrm{N} 2 \mathrm{C}$ & 16 & 11.4 & 8 & 10.5 & & \\
\hline N3 & 3 & 2.1 & 0 & 0 & & \\
\hline \multicolumn{7}{|l|}{ TNM stage $(n, \%)$} \\
\hline 1 & 20 & 14.3 & 7 & 9.2 & 2.38 & 0.51 \\
\hline$\|$ & 29 & 20.7 & 14 & 18.4 & & \\
\hline III & 26 & 18.6 & 12 & 15.8 & & \\
\hline IV & 65 & 46.4 & 43 & 56.6 & & \\
\hline \multicolumn{7}{|l|}{ Chemotherapy $(n, \%)$} \\
\hline No & 95 & 67.9 & 41 & 53.9 & 4.86 & 0.09 \\
\hline Primary & 40 & 28.6 & 33 & 43.4 & & \\
\hline Adjuvant & 5 & 3.6 & 2 & 2.6 & & \\
\hline \multicolumn{7}{|l|}{ Radiotherapy (n, \%) } \\
\hline No & 18 & 12.9 & 5 & 6.6 & 2.41 & 0.29 \\
\hline Primary & 113 & 80.7 & 64 & 84.2 & & \\
\hline Adjuvant & 9 & 6.4 & 7 & 9.2 & & \\
\hline \multicolumn{7}{|l|}{ Surgery $(\mathrm{n}, \%)$} \\
\hline No & 110 & 78.6 & 61 & 80.3 & 0.06 & 0.86 \\
\hline Yes & 30 & 21.4 & 15 & 19.7 & & \\
\hline
\end{tabular}




\section{Risk-stratification according to HPV-status}

Figure 2 shows the Kaplan Meier curves and number at risk tables of overall survival and disease-free survival for patients stratified into risk groups. Patients within the high-risk group had a statistically significant worse median OS and DFS (27 months; IQR 12-50 and 20 months; IQR 9-46 respectively) compared to patients within an intermediate risk group (47 months; IQR 38-63 and 47 months; IQR 2663 respectively) and low risk group (45 months; IQR 22-62 and 44 months; IQR 16-62 respectively) (high versus intermediate risk: Log Rank $x^{2}=20.02 ; p<0.01$ and high versus low risk: $\left.\log \operatorname{Rank} x^{2}=16.61 ; p<0.01\right)$. Patients within a high-risk group had a significantly worse 5 -year OS and DFS rate (32\% and $50 \%$, respectively) compared to patients within a intermediate risk group ( $74 \%$ and $80 \%$, respectively) $(p<0.01)$ and low risk group $(72 \%$ and $87 \%$, respectively) $(p<0.01)$.

As shown in Table 2 and Table 3; univariate Cox regression analysis showed that stratification into a high-risk group was statistically significant associated with a decreased OS (HR 3.11; 95\% Cl 1.61-6.01, $\mathrm{p}<0.01$ ) and DFS (HR 4.85; 95\% Cl 1.89$12.42, \mathrm{p}<0.01)$. When corrected for multiple, potentially interacting variables by multivariate Cox regression analysis; stratification into a high-risk group remained of significant negative prognostic value for OS (HR 2.31; 95\% Cl 1.14-4.68, $\mathrm{p}<0.05$ ) and DFS (HR 4.06; 95\% Cl 1.52-10.84, $\mathrm{p}<0.01$ ).

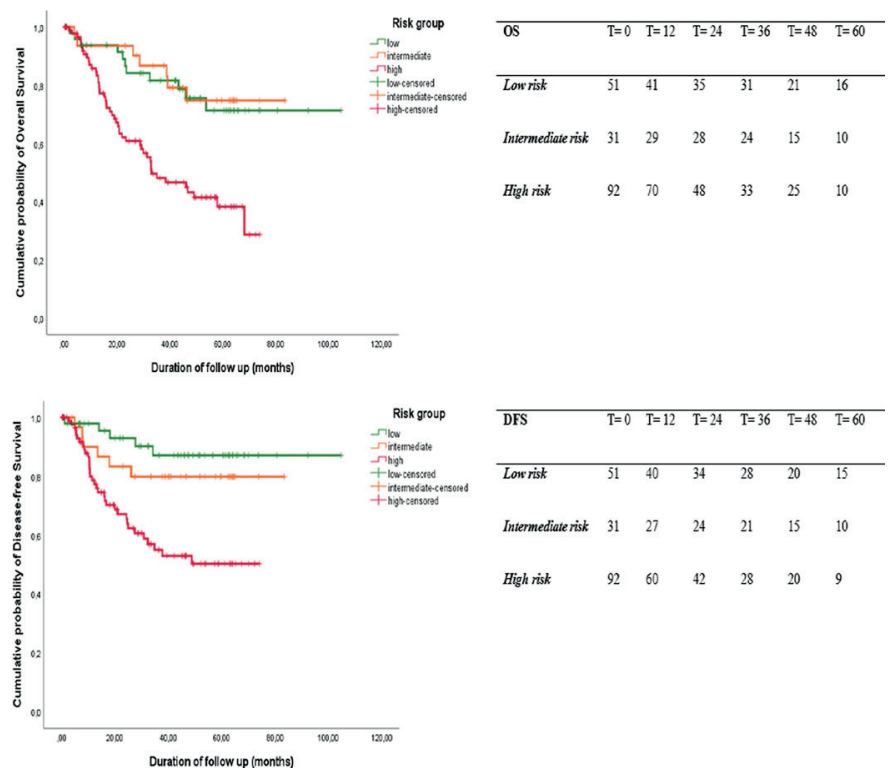

Figure 2. Kaplan Meier curves and number at risk tables for overall survival (top) and disease-free survival (bottom) in OPSCC patients stratified to low, intermediate and high-risk groups shows significant difference in OS (Log Rank X2 $=20.02, p<0.01)$ and DFS (Log Rank $x 2=16.61, p<0.01)$.
Table 2. Univariate and multivariate Cox regression analysis for analyzing variables associated with overall survival in OPSCC patients

\begin{tabular}{|c|c|c|c|c|c|c|c|c|c|}
\hline \multirow[t]{3}{*}{ Variable } & \multicolumn{3}{|c|}{ Univariate analysis } & \multicolumn{6}{|c|}{ Multivariate analysis $(*)$} \\
\hline & \multirow[b]{2}{*}{ HR } & \multirow[b]{2}{*}{$95 \% \mathrm{Cl}$} & \multirow[b]{2}{*}{ P-value } & \multicolumn{3}{|c|}{ Model 1} & \multicolumn{3}{|c|}{ Model 2} \\
\hline & & & & HR & $95 \% \mathrm{Cl}$ & $\begin{array}{l}\text { P-value } \\
\end{array}$ & HR & $95 \% \mathrm{Cl}$ & $\begin{array}{l}\text { P-value } \\
\end{array}$ \\
\hline Low SMM & 1.76 & $1.02-3.04$ & $0.04^{*}$ & 1.28 & $0.68-2.22$ & 0.50 & - & - & - \\
\hline Sarcopenic obesity & 2.44 & $1.05-5.69$ & $0.04^{*}$ & - & - & - & 3.16 & $1.31-7.63$ & $0.01^{*}$ \\
\hline \multicolumn{10}{|l|}{ Risk group } \\
\hline Low & Ref. & & & Ref. & & & Ref. & & \\
\hline Intermediate & 0.90 & $0.35-2.31$ & 0.82 & 0.81 & $0.31-2.11$ & 0.66 & 0.882 .56 & $0.34-2.30$ & 0.79 \\
\hline High & 3.11 & $1.61-6.01$ & $0.001^{* *}$ & 2.31 & $1.14-4.68$ & $0.02^{*}$ & & $1.30-5.06$ & $0.007^{* *}$ \\
\hline Age (years) & 1.02 & $0.99-1.04$ & 0.17 & & & & & & \\
\hline \multicolumn{10}{|l|}{ BMI $\left(\mathbf{k g} / \mathbf{m}^{2}\right)$} \\
\hline $20-24.9$ & Ref. & & & & & & & & \\
\hline$<20$ & 1.36 & $0.73-2.54$ & 0.33 & & & & & & \\
\hline $25-30$ & 0.56 & $0.29-1.09$ & 0.09 & & & & & & \\
\hline$\geq 30$ & 0.77 & $0.35-1.68$ & 0.51 & & & & & & \\
\hline \multicolumn{10}{|c|}{$\begin{array}{l}\text { Weight loss } 6 \text { months prior to } \\
\text { diagnosis }(\%)\end{array}$} \\
\hline$<10 \%$ & Ref. & & & Ref. & & & Ref. & & \\
\hline \multirow[t]{2}{*}{$\geq 10 \%$} & 1.56 & $0.85-2.85$ & 0.15 & 1.43 & $0.76-2.69$ & 0.27 & 1.62 & $0.86-3.05$ & 0.14 \\
\hline & 3.28 & $1.76-6.11$ & $0.0001^{* *}$ & 2.62 & $1.38-4.98$ & $0.003^{* *}$ & 3.05 & $1.61-5.78$ & $0.001^{* *}$ \\
\hline \multicolumn{10}{|l|}{ ACE-27 score } \\
\hline Non & Ref. & & & Ref. & & & Ref. & & \\
\hline Mild & 2.08 & $0.93-4.68$ & 0.08 & 1.97 & $0.87-4.45$ & 0.10 & 1.95 & $0.86-4.41$ & 0.11 \\
\hline Moderate & 2.97 & $1.38-6.36$ & $0.005^{* *}$ & 2.43 & $1.12-5.29$ & $0.03^{*}$ & 2.27 & $1,04-4.92$ & $0.04^{*}$ \\
\hline Severe & 4.74 & $2.05-10.98$ & $0.0001^{* *}$ & 4.08 & 1.75-9.52 & $0.001 * *$ & 3.86 & $1.65-9.06$ & $0.002^{* *}$ \\
\hline \multicolumn{10}{|l|}{ TNM stage } \\
\hline 1 & Ref. & & & & & & & & \\
\hline$\|$ & 0.94 & $0.28-3.21$ & 0.92 & & & & & & \\
\hline III & 2.39 & $0.79-7.25$ & 0.13 & & & & & & \\
\hline IV & 2.10 & $0.75-5.86$ & 0.16 & & & & & & \\
\hline
\end{tabular}

** Correlation is significant at the 0.01 level (2-tailed)

* Correlation is significant at the 0.05 level (2-tailed)

(*) Model 1 includes the variables; low SMM (SMM), HPV-related risk group, age, BMI, percentage weight loss 6 months prior to diagnosis, ACE-27 score and TNM-stage. Model 2 includes sarcopenic obesity, HPV-related risk group, age, BMI, percentage weight 6 months prior to diagnosis, ACE-27 score and TNM-stage. 
Table 3. Univariate and multivariate Cox regression analysis: disease-free survival

\begin{tabular}{|c|c|c|c|c|c|c|c|c|c|}
\hline \multirow[t]{3}{*}{ Variable } & \multicolumn{3}{|c|}{ Univariate analysis } & \multicolumn{6}{|c|}{ Multivariate analysis ( $*$ ) } \\
\hline & \multirow[b]{2}{*}{ HR } & \multirow[b]{2}{*}{$95 \% \mathrm{Cl}$} & \multirow[b]{2}{*}{ P-value } & \multicolumn{3}{|c|}{ Model 1} & \multicolumn{3}{|c|}{ Model 2} \\
\hline & & & & HR & $95 \% \mathrm{Cl}$ & P-value & HR & $95 \% \mathrm{Cl}$ & $\begin{array}{l}\text { P-value } \\
\end{array}$ \\
\hline Low SMM & 1.81 & $0.95-3.45$ & 0.07 & 1.41 & $0.60-3.33$ & 0.44 & - & - & - \\
\hline Sarcopenic obesity & 2.03 & $0.73-5.68$ & 0.18 & - & - & - & 3.49 & $1.08-11.27$ & $0.037^{*}$ \\
\hline \multicolumn{10}{|l|}{ HPV risk group } \\
\hline & Ref. & & & Ref. & & & Ref. & & \\
\hline Intermediate & 1.76 & $0.54-5.77$ & 0.35 & 1.71 & $0.51-5.72$ & 0.38 & 1.93 & $0.59-6.36$ & $0.280 .003^{* *}$ \\
\hline High & 4.85 & $1.89-12.42$ & $0.001 * *$ & 4.06 & $1.52-10.84$ & $0.005 * *$ & 4.32 & $1.64-11.38$ & \\
\hline Age (years) & 0.99 & $0.96-1.02$ & 0.57 & & & & & & \\
\hline \multicolumn{10}{|l|}{ BMI (kg/m²) } \\
\hline $20-24.9$ & Ref. & & & Ref. & & & Ref. & & \\
\hline$<20$ & 1.05 & $0.48-2.29$ & 0.90 & 0.75 & $0.34-1.64$ & 0.75 & 0.78 & $0.36-1.72$ & 0.54 \\
\hline $25-30$ & 0.36 & $0.14-0.88$ & $0.03^{*}$ & 0.541 .30 & $0.21-1.42$ & 0.21 & 0.39 & $0.14-1.05$ & 0.06 \\
\hline$\geq 30$ & 0.96 & $0.43-2.15$ & 0.92 & & $0.47-3.56$ & 0.62 & 0.80 & 0.33-1.94 & 0.62 \\
\hline \multicolumn{10}{|c|}{$\begin{array}{l}\text { Weight loss } 6 \text { months prior } \\
\text { to diagnosis (\%) }\end{array}$} \\
\hline \multicolumn{10}{|l|}{0} \\
\hline$<10 \%$ & Ref. & & & & & & & & \\
\hline \multirow[t]{2}{*}{$>10 \%$} & 1.46 & $0.73-2.91$ & 0.29 & & & & & & \\
\hline & 1.33 & $0.52-3.45$ & 0.55 & & & & & & \\
\hline \multicolumn{10}{|l|}{ ACE-27 score } \\
\hline Non & Ref. & & & & & & & & \\
\hline Mild & 1.02 & $0.45-2.31$ & 0.97 & & & & & & \\
\hline Moderate & 1.42 & $0.68-2.97$ & 0.36 & & & & & & \\
\hline Severe & 1.14 & $0.40-3.25$ & 0.80 & & & & & & \\
\hline \multicolumn{10}{|l|}{ TNM stage } \\
\hline I & Ref. & & & & & & & & \\
\hline ॥ & 0.70 & $0.21-2.28$ & 0.55 & & & & & & \\
\hline III & 1.19 & $0.39-3.64$ & 0.76 & & & & & & \\
\hline IV & 1.13 & $0.43-2.94$ & 0.81 & & & & & & \\
\hline \multicolumn{10}{|c|}{ ** Correlation is significant at the 0.01 level (2-tailed) } \\
\hline \multicolumn{10}{|c|}{ * Correlation is significant at the 0.05 level (2-tailed) } \\
\hline \multirow{2}{*}{\multicolumn{10}{|c|}{$\begin{array}{l}\text { (*) Model } 1 \text { includes the variables; low SMM, HPV-related risk group, age, BMI, percentage weight } \\
\text { loss } 6 \text { months prior to diagnosis, ACE-27 score and TNM-stage. Model } 2 \text { includes sarcopenic obesity, }\end{array}$}} \\
\hline & & & & & & & & & \\
\hline HPV-related $\mathrm{ri}$ & 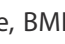 & centage & ight lo & s6 mor & prior to & agno & $\mathrm{ACE}$ & score & \\
\hline
\end{tabular}

Clinical and demographic characteristics of patients with and without low SMM are listed in Table 1. Statistically significant differences between patients with and without low SMM were seen in sex, age at diagnosis, percentage of weight loss within six months prior to diagnosis, body-mass-index, amount of packyears, HPV-status and HPV risk group. Patients with low SMM were more likely to be female $(50.7 \%$ versus $2.6 \% ; \mathrm{p}<0.01)$, to be older of age at diagnosis (mean 63.6 years versus 60.3 years; $p<0.05$ ), to have $10 \%$ or more weight loss in the six months prior to diagnosis ( $15 \%$ versus $7.9 \%, \mathrm{p}<0.05)$, to have a BMI that is less than $20 \mathrm{~kg} / \mathrm{m}^{2}$ (28.6\% versus $\left.2.6 \% ; \mathrm{p}<0.01\right)$, to have smoked for more than 41 packyears $(40.3 \%$ versus $22.2 \% ; \mathrm{p}<0.05)$, to have a HPV-negative related tumor $(55 \%$ versus $36.8 \% ; p<0.01)$ and to be stratified within a high-risk group $(39.1 \%$ versus $13.8 \% ; p<0.01)$

Figure 3 shows the Kaplan Meier survival curves and number at risk tables of overall and disease-free survival for OPSCC patients with and without low SMM Patients with low SMM had a significant lower median OS (32.74; IQR 12.72-53.70) compared to patients without low SMM (42.05; IQR 23.61-62.32) (Log rank X2= 4.30; $p=0.04$ ) with a 5 -year OS rate of $43 \%$ versus $66 \%$. Patients with low SMM showed a statistical trend towards lower median DFS rate (24.72 months; IQR 9.4251.78) compared to patients without low SMM (35.25 months; IQR 15.93-62.10) (Log rank $\mathrm{x} 2=3.35, \mathrm{p}=0.07$ ) with a 5 -year DFS rate of $61 \%$ versus $76 \%$.

Figure 4 shows the Kaplan Meier survival curves and number at risk tables of overall and disease-free survival for OPSCC patients with and without sarcopenic obesity. Patients with sarcopenic obesity had a significant lower median OS (23.03; IQR 4.90-32.82) compared to patients without sarcopenic obesity (38.74; IQR 16.00-57.92) (Log rank $x 2=4.60 ; p=0.03$ ) with a 3 -years OS rate of $39 \%$ versus $60 \%$. Patients with sarcopenic obesity showed lower median DFS rate (23.66 months; IQR 5.48-33.35) compared to patients without sarcopenic obesity (32.35 months; IQR 10.60-57.08) (Log rank $X 2=1.90, p=0.17)$ with a 3 -year DFS rate of $51 \%$ versus $70 \%$.

\section{Body composition}

Endpoint-specific cutoff values for $\mathrm{L} 3$ muscle mass indices were determined at $43.0 \mathrm{~cm}^{2} / \mathrm{m}^{2}$ for OS and $43.2 \mathrm{~cm}^{2} / \mathrm{m}^{2}$ for DFS. These cut-off values are comparably with previous cutoff values established in a separate cohort of head and neck cancer patients. ${ }^{20}$ Using these cutoff values, 140 patients (64.8\%) were identified with low SMM and 13 patients (6\%) were identified with sarcopenic obesity. 

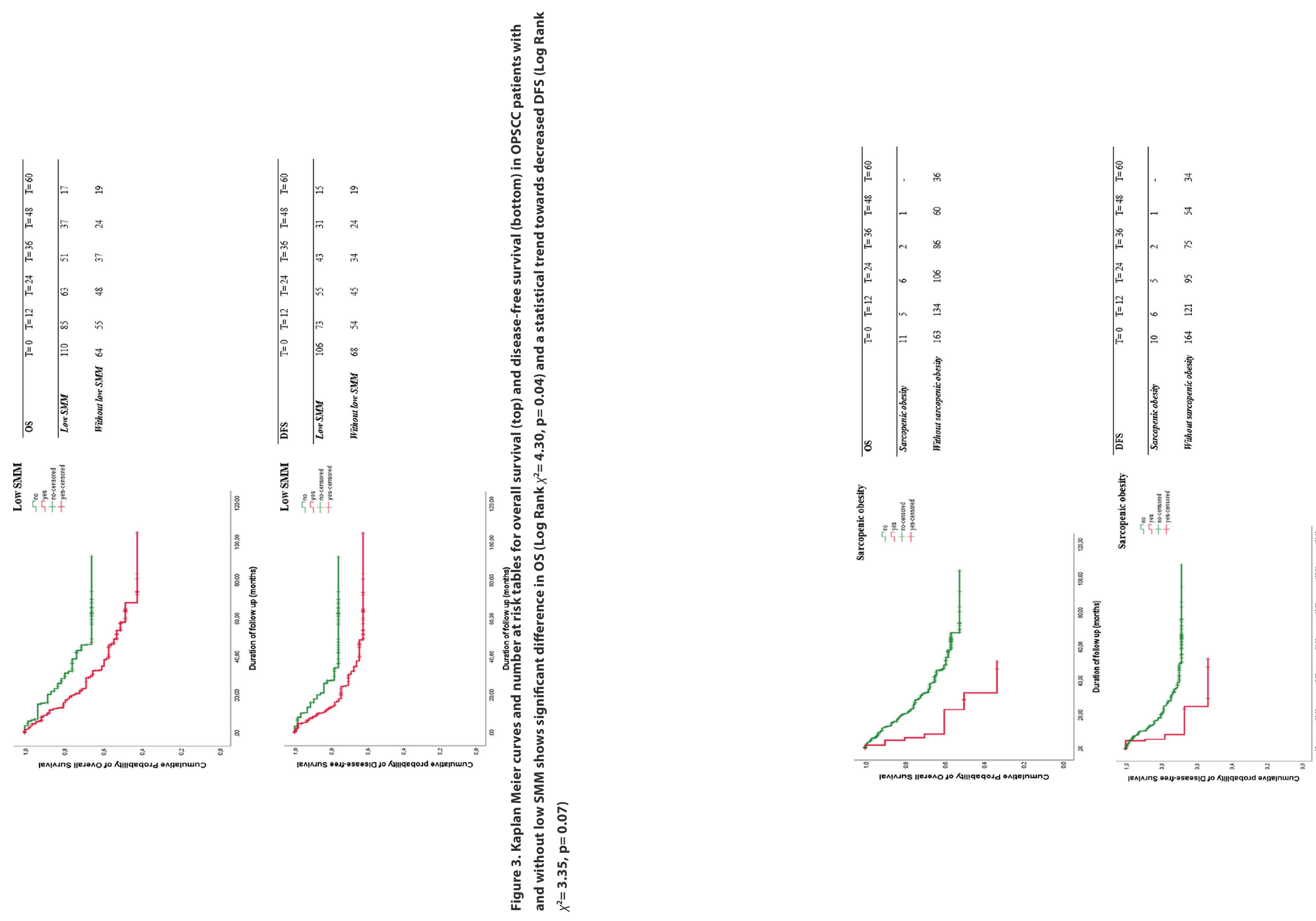

焉

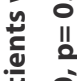

产

능

흘

है

을 
As shown in Table 2 and Table 3, univariate Cox regression analysis showed that low SMM and sarcopenic obesity were statistically significant associated with a decreased OS (HR 1.76; 95\% Cl 1.02-3.04, $\mathrm{p}=0.04$ and HR 2.44; 95\% Cl 1.05-5.69, $\mathrm{p}=0.04$, respectively), but not with a decreased DFS (HR 1.81; 95\% Cl 0.95-3.45, $\mathrm{p}=0.07$ and $\mathrm{HR} 2.03 ; 95 \% \mathrm{Cl} 0.73-5.68, \mathrm{p}=0.18$, respectively). In multivariate Cox regression analysis, sarcopenic obesity remained a significant negative prognostic factor for OS (HR 3.16; 95\% Cl 1.31-7.63, $\mathrm{p}<0.05$ ) and became a significant negative prognostic for DFS (HR 3.49; $95 \% \mathrm{Cl} 1.08-11.27, \mathrm{p}<0.05$ ). For OS, this result was independent of HPV-related risk group, percentage of weight loss 6 months prior to diagnosis and comorbidity as assessed by the ACE-27 score and for DFS this result was independent of HPV-related risk group and BMI.

\section{Discussion}

The worldwide incidence of OPSCC is increasing, as is the prevalence of HPVpositive status in OPSCC. The most important prognostic factor in OPSCC is HPVstatus; patients with HPV-positive OPSCC have a vastly better prognosis than patients with HPV-negative disease. Over the last decade, low SMM and sarcopenic obesity have emerged as negative prognostic factors in a variety of cancer types and stages. ${ }^{17} 1530$

This study shows that low SMM, sarcopenic obesity and stratification into a highrisk group are associated with impaired survival rates in patients with OPSCC; sarcopenic obesity especially is a negative prognostic factor for overall and diseasefree survival in OPSCC, independent from HPV-status and other factors such as age, $\mathrm{BMI}$, percentage of weight loss 6 months prior to diagnosis, comorbidities and TNMstage. Pre-treatment low SMM is highly prevalent in patients with OPSCC with an incidence of $64.8 \%$. In contrast, sarcopenic obesity is rare, and occurs in only $6.0 \%$ of patients with OPSCC.

The individual body composition of cancer patients is increasingly recognized as an important predictive factor for treatment tolerance and for survival after treatment. Specifically, an abnormal body composition with a deficit of SMM with or without a surplus of fat mass (sarcopenia and sarcopenic obesity), is associated with adverse outcomes in oncological patients ${ }^{31}$. Studies in patients with gastrointestinal cancer ${ }^{32}$ , lung cancer ${ }^{33}$, breast cancer ${ }^{34}$ and pancreatic cancer have shown that patients with sarcopenia or sarcopenic obesity appear to be more prone to experience toxicity of chemotherapeutical treatment and to suffer from complications after surgery.
In head and neck cancer patients, recent studies have shown that there is an association between pre-treatment low SMM and chemotherapy dose-limiting toxicity ${ }^{20}$, complications and pharyngocutaneous fistula after total laryngectomy ${ }^{21}$ ${ }^{22}$, and decreased overall survival. ${ }^{35}$ Regarding chemotherapy related toxicity, a hypothesis for this relationship is that patients with low SMM and sarcopenic obesity have a different distribution of chemotherapeutical agents in the body. In terms of complications after surgery, it is hypothesized that patients with sarcopenia may have a decreased capability for recovery, for instance due to an altered protein metabolism or a decreased physiological reserve to deal with surgical stress.

In a recent study in advanced oropharyngeal cancer patients, pre-treatment low SMM as a negative prognostic factor in patients with HPV-positive and HPVnegative oropharyngeal cancer showed a trend towards statistical significance. ${ }^{23}$ Our study in a larger unselected cohort of OPSCC patients concurs with these results and adds information on the prevalence and prognostic value of sarcopenic obesity in relation to a previous published HPV-related risk stratification model in OPSCC patients. It shows that sarcopenia is highly prevalent in OPSCC patients prior to start of treatment, possibly because oropharyngeal tumors have a high risk of causing dysphagia. ${ }^{30}$

The exact mechanisms of sarcopenia and its relationship with adverse outcomes are currently unknown. It is also unknown to which extent the negative effect of sarcopenia can be overturned by improving a patient's physical condition and nutritional status before and during treatment. Future research is needed to clarify these mechanisms. Treatment strategies may be personalized to the patient's specific body composition to decrease the risk of severe toxicity and adverse outcomes, while still maintaining optimal efficacy.

A limitation of this study is the retrospective design which increases the risk of systemic errors and missing data. For example, HPV-status was not available in all patients in this cohort; thus, for survival analysis, patients without a known HPV-status were excluded. Another limitation is that low SMM was not defined according to sex-specific cut-offs, which may result in an overrepresentation of women in the low SMM group. When more data of female HNSCC patients is available, we aim to define sex-specific cut-offs for low SMM in HNSCC patients. Another limitation is that survival was not measured by treatment modality due to heterogeneity and variations between- and within treatment modalities. Further research is needed to investigate the role of low SMM on survival in patients treated with different treatment modalities. 
Our recently published measurement method for SMM at the level of C3 allows for the routine evaluation of sarcopenia in almost all head and neck cancer patients. In the future, this tool may be used as a screening tool for patients at risk of severe toxicity or complications from treatment.

This study has examined the prevalence and prognostic value of low SMM and sarcopenic obesity, while adjusting for a variety of known confounders (e.g. comorbidity, weight loss, BMI, HPV-status, TNM-stage), in a large cohort of OPSCC patients. The findings in this study highlight the potential usefulness of determining pre-treatment SMM in HNC patients and contributes to a growing knowledge of low SMM and sarcopenic obesity in HNSCC patients. This knowledge can be used for the development of new interventions, patient management and treatment decision making. In addition, this information can be used for the development of improved risk stratification models in OPSCC patients and deintensification approaches in HPV-related OPSCC.

\section{Conclusion}

Pre-treatment low skeletal muscle mass is highly prevalent in patients with oropharyngeal squamous cell carcinoma. The simultaneous presence of low skeletal muscle mass and obesity, sarcopenic obesity, has a statistically significant association with decreased overall and disease-free survival, independent from other well-known prognostic factors such as HPV-status. Therefore, skeletal muscle mass should be considered as a pre-treatment prognostic factor in clinical decision making.

\section{References}

1. Bray F, Ferlay J, Soerjomataram I, Siegel RL, Torre LA, Jemal A. Global cancer statistics 2018 GLOBOCAN estimates of incidence and mortality worldwide for 36 cancers in 185 countries. CA Cancer J Clin. 2018;68(6):394-424.

2. Argiris A, Eng C. Epidemiology, staging, and screening of head and neck cancer. Cancer Treat Res. 2003; 114:15-60.

3. Hashibe M, Brennan P, Chuang SC, et al. Interaction between tobacco and alcohol use and the risk of head and neck cancer: Pooled analysis in the international head and neck cancer epidemiology consortium. Cancer Epidemiol Biomarkers Prev. 2009;18(2):541-550.

4. Mehanna H, Beech T, Nicholson T, et al. Prevalence of human papillomavirus in oropharyngeal and nonoropharyngeal head and neck cancer--systematic review and meta-analysis of trends by time and region. Head Neck. 2013;35(5):747-755.

5. Windon MJ, D'Souza G, Rettig EM, et al. Increasing prevalence of human papillomaviruspositive oropharyngeal cancers among older adults. Cancer. 2018;124(14):2993-2999.

6. Anantharaman D, Billot A, Waterboer T, et al. Predictors of oropharyngeal cancer survival in europe. Oral Oncol. 2018;81:89-94.

7. Habbous S, Harland LT, La Delfa A, et al. Comorbidity and prognosis in head and neck cancers: Differences by subsite, stage, and human papillomavirus status. Head Neck. 2014;36(6):802810.

8. Le Tourneau C, Velten M, Jung GM, Bronner G, Flesch H, Borel C. Prognostic indicators for survival in head and neck squamous cell carcinomas: Analysis of a series of 621 cases. Head Neck. 2005;27(9):801-808.

9. Ang KK, Harris J, Wheeler R, et al. Human papillomavirus and survival of patients with oropharyngeal cancer. N Engl J Med. 2010;363(1):24-35.

10. Dahlstrom KR, Garden AS, William WN,Jr, Lim MY, Sturgis EM. Proposed staging system for patients with HPV-related oropharyngeal cancer based on nasopharyngeal cancer $N$ categories. J Clin Oncol. 2016;34(16):1848-1854.

11. Huang $\mathrm{SH}, \mathrm{Xu} \mathrm{W}$, Waldron J, et al. Refining american joint committee on cancer/union for international cancer control TNM stage and prognostic groups for human papillomavirusrelated oropharyngeal carcinomas. J Clin Oncol. 2015;33(8):836-845.

12. O'Sullivan B1, Huang SH, Siu LL, Waldron J, Zhao H, Perez-Ordonez B, Weinreb I, Kim J, Ringash J, Bayley A, Dawson LA, Hope A, Cho J, Irish J, Gilbert R, Gullane P, Hui A, Liu FF, Chen E, Xu $W$. Deintensification candidate subgroups in human papillomavirus-related oropharyngea cancer according to minimal risk of distant metastasis. J Clin Oncol. $2013 \mathrm{Feb}$ 10:31(5):543-50

Daly LE Prado CM Ryan AM. A window beneath the skin: How computed tomography assessment of body composition can assist in the identifcation of hidden wasting conditions

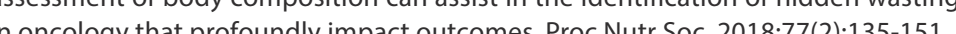

14. Cruz-Jentoft AJ, Baeyens JP, Bauer JM, et al. Sarcopenia: European consensus on definition and diagnosis: Report of the european working group on sarcopenia in older people. Age Ageing 2010;39(4):412-423.

15. Prado $\mathrm{CM}$, Lieffers JR, McCargar $\mathrm{LJ}$, et al. Prevalence and clinical implications of sarcopenic obesity in patients with solid tumours of the respiratory and gastrointestinal tracts: A population-based study. Lancet Oncol. 2008;9(7):629-635.

16. Carneiro IP, Mazurak VC, Prado CM. Clinical implications of sarcopenic obesity in cancer. Curr Oncol Rep. 2016;18(10):5.

17. Rier $H N$, Jager $A$, Sleijfer $S$, Maier AB, Levin MD. The prevalence and prognostic value of low muscle mass in cancer patients: A review of the literature. Oncologist. 2016;21(11):1396-1409. 8. Levolger $S$, van Vugt $J L$, de Bruin RW, IJzermans JN. Systematic review of sarcopenia in patients operated on for gastrointestinal and hepatopancreatobiliary malignancies. Br J Surg 2015;102(12):1448-1458.

19. Pamoukdjian F, Bouillet T, Levy V, Soussan M, Zelek L, Paillaud E. Prevalence and predictive value of pre-therapeutic sarcopenia in cancer patients: A systematic review. Clin Nutr. 2018;37(4):1101-1113. 
20. Wendrich AW, Swartz JE, Bril Sl, et al. Low SMM is a predictive factor for chemotherapy dose-limiting toxicity in patients with locally advanced head and neck cancer. Oral Oncol.

21. Achim V, Bash J, Mowery A, et al. Prognostic indication of sarcopenia for wound complication after total laryngectomy. JAMA Otolaryngol Head Neck Surg. 2017;143(12):1159-1165.

22. Bril Sl, Pezier TF, Tijink BM, Janssen LM, Braunius WW, de Bree R. Preoperative low skeletal muscle mass as a risk factor for pharyngocutaneous fistula and decreased overall survival in

23. Tamaki A, Manzoor NF, Babajanian E, Ascha M, Rezaee R, Zender CA. Clinical significance of sarcopenia among patients with advanced oropharyngeal cancer. Otolaryngol Head Neck Surg. 2018:194599818793857.

24. Gregoire V, Lefebvre JL, Licitra L, Felip E, EHNS-ESMO-ESTRO Guidelines Working Group. Squamous cell carcinoma of the head and neck: EHNS-ESMO-ESTRO clinical practice guidelines for diagnosis, treatment and follow-up. Ann Oncol. 2010;21 Suppl 5:184.

25. Swartz JE, Pothen AJ, Wegner $\mathrm{l}$, et al. Feasibility of using head and neck CT imaging to assess skeletal muscle mass in head and neck cancer patients. Oral Oncol. 2016;62:28-33,

26. Bril Sl, Wendrich AW, Swartz JE, et al. Interobserver agreement of skeletal muscle mass measurement on head and neck CT imaging at the level of the third cervical vertebra. Eur Arch Otorhinolaryngol. 2019,

27. Smeets SJ, Hesselnk AT, Speel EJ, et al. A novel algorithm for reliable detection of human papillomavirus in paraffin embedded head and neck cancer specimen. Int J Cancer. 2007;121(11):2465-2472.

28. Heymsfield SB, Wang Z, Baumgartner RN, Ross R. Human body composition: Advances in models and methods. Annu Rev Nutr. 1997;17:527-558.

29. Williams BA, Mandrekar JA, Mandrekar SJ, Cha SS, Furth AF. Finding optimal cutpoints for continuous covariates with binary and time-to-event outcomes. Mayo Foundation technica report series \#79. 2006.

30. Carneiro IP, Mazurak Ve, Prado CM. Clinical implications of sarcopenic obesity in cancer. Curr Oncol Rep. 2016;18(10):5.

31. Baracos VE, Arribas L. Sarcopenic obesity: Hidden muscle wasting and its impact for survival and complications of cancer therapy. Ann Oncol. 2018;29(suppl_2):iig.

32. Palmela C, Velho S, Agostinho L, et al. Body composition as a prognostic factor of neoadjuvant chemotherapy toxicity and outcome in patients with locally advanced gastric cancer. J Gastric Cancer. 2017;17(1):74-87.

33. Sjoblom B, Benth JS, Gronberg BH, et al. Drug dose per kilogram lean body mass predicts hematologic toxicity from carboplatin-doublet chemotherapy in advanced non-small-cell ung cancer. Clin Lung Cancer. 2017;18(2):e136.

34. Prado CM, Baracos VE, McCargar LJ, et al. Sarcopenia as a determinant of chemotherapy toxicity and time to tumor progression in metastatic breast cancer patients receiving capecitabine treatment. Clin Cancer Res. 2009;15(8):2920-2926.

35. Chargi N, Bril Sl, Emmelot-Vonk MH, de Bree R. Sarcopenia is a prognostic factor for overall survival in elderly patients with head-and-neck cancer. Eur Arch Otorhinolaryngol. 2019;276(5):1475-1486. 


\section{Chapter 9}

Arterial calcification on preoperative $\mathrm{CT}$ imaging is a risk factor for pharyngocutaneous fistula formation after total laryngectomy

Sandra I. Bril, Najiba Chargi, Thomas F. Pezier, Bernard M. Tijink, Weibel W. Braunius, Ernst J. Smid, Pim A. de Jong, Remco de Bree 


\section{Abstract}

Background Research in oesophageal surgery showed that CT assessed arterial calcification $(A C)$ is associated with postoperative complications. We investigated the association between $\mathrm{AC}$ and pharyngocutaneous fistula (PCF) formation after laryngectomy.

Methods This was a retrospective cohort study of patients undergoing laryngectomy. AC was scored on CT imaging, blinded for PCF occurrence. Association with PCF was investigated using logistic regression.

Results 224 patients were included; 62 (27.7\%) developed a PCF. 7.1\% of patients had at most mild AC, of whom 1 experienced a PCF $(p=0.05)$. Moderate to severe $A C$ of the descending aorta, origins of brachiocephalic arteries and left carotid siphon was associated with PCF (OR 2.07-2.83; all $\mathrm{p}<0.05)$. A higher cumulative calcification score was associated with PCF (adjusted OR 1.06-1.08; $p<0.05$ ).

Conclusion $\mathrm{AC}$ is widespread in patients undergoing laryngectomy and its burden is associated with PCF. Extensive AC on preoperative imaging may be considered a risk factor for $P C F$

\section{Introduction}

Total laryngectomy (TL) is a definitive treatment for patients with advanced stage laryngeal or pharyngeal cancer. It is also a salvage treatment option for patients with recurrent disease after (chemo)radiotherapy, and can be used to treat a dysfunctional larynx., It is an invasive surgical procedure and associated with frequent postoperative complications resulting in significant morbidity and mortality, compromising survival and quality of life..$^{3-5}$

Postoperative complications, including wound healing problems and the occurrence of a pharyngocutaneous fistula (PCF), are common and difficult to treat. Approximately $30 \%$ of patients develops a PCF after TL, which often requires additional surgery, flap reconstruction, prolonged hospital stay and feeding tube dependency. ${ }^{6,7}$ Known risk factors for PCF are prior chemoradiotherapy, hypopharyngeal cancer, extensive pharyngeal resection and reconstruction, neck dissection, and low body mass index (BMI). Recently, radiologically assessed sarcopenia or low skeletal muscle mass has been identified as a novel risk factor for PCF in patients undergoing total laryngectomy. ${ }^{8,9}$

Research shows that routinely performed imaging, such as computed tomography (CT), can be used to extract additional information on patient's body composition as a biomarker of functional and biological status, next to cancer specific features and risk factors. ${ }^{10-12}$ The radiological assessment of sarcopenia is an example of this. Routinely performed CT imaging can also be used to assess arteria calcification, as evidence of the presence of atherosclerosis. ${ }^{13,14}$ In head and neck cancer patients, head and neck CT imaging is commonly performed during the diagnostic work-up, on which the carotid arteries and vertebral arteries are shown. Additionally, thoracic CT imaging and/or whole body FDG-PET/CT imaging may be performed, depending on local diagnostic protocols, which provides additional imaging of the heart and aorta. Smoking, a known etiological factor for atherosclerosis, is common in head and neck cancer patients. ${ }^{15}$ Another etiological factor for atherosclerosis is a low-level persistent systemic inflammation, which is also commonly observed in cancer patients. ${ }^{16,17}$ It may be hypothesized that atherosclerosis is a risk factor for wound healing in surgery due to inadequate perfusion at the site of surgery and microvascular dysfunction Recent studies in patients with oesophageal cancer undergoing esophagectomy showed that locoregional and generalized cardiovascular disease as identified on routine CT imaging was predictive of cervical anastomotic leakage. ${ }^{18,19}$ Another study in patients with rectal cancer showed that calcification of the iliac arteries 
was associated with anastomotic leakage after colorectal surgery with recta anastomosis. $^{20}$

The purpose of this study was to explore the extent of arterial calcifications present in patients undergoing $\mathrm{TL}$, investigate whether the presence and burden of regional and generalized atherosclerotic calcification, as visualized on preoperative $\mathrm{CT}$ imaging is a risk factor for PCF in patients undergoing TL.

\section{Patients and methods}

This study is a retrospective cohort study on which we previously reported. ${ }^{8}$ The design of this study was approved by the Medical Ethical Research Committee of the University Medical Center Utrecht (ID 17-365/C). The research was conducted in accordance with the Declaration of Helsinki.

\section{Patient and study design}

All patients who had undergone TL between January 2008 and May 2017 at our institution were considered for inclusion. Patients were discussed in the local tumor board meeting, and all patients who were included underwent TL with or without (partial) pharyngectomy and with or without additional lymph node dissection; either as primary treatment, as salvage treatment for recurrent or residual tumor after prior (chemo)radiotherapy treatment, or as functional treatment for a dysfunctional larynx after prior (chemo)radiotherapy, where no vital tumor was found. Five dedicated head and neck surgical oncologists performed all TL. Exclusion criteria for this analysis included insufficient quality $\mathrm{CT}$ imaging as determined by an experienced radiologist or the absence of $\mathrm{CT}$ imaging (e.g. only MRI imaging performed).

Patients' demographic, tumor stage, treatment, and outcome data were collected using electronic patient records. Operating records were checked for details of the surgery, neck dissection, and primary pharyngeal closure or flap reconstruction of the pharynx. The occurrence of PCF was defined as a clinical fistula requiring conservative or surgical treatment. In patients who had surgery for a dysfunctiona larynx, the tumor site for which the patient received prior treatment was documented. Follow-up data were retrieved up until August 31, 2017.

The presence of sarcopenia was assessed on preoperative CT imaging using a previously specified protocol. In brief, the cross-sectional skeletal muscle area at the level of C3 was measured on a single transversal CT slice at the level of the third cervical vertebra (C3) ${ }^{8,12}$ The cross-sectional muscle area was normalized for height to calculate the skeletal muscle index. A skeletal muscle index of below $43.2 \mathrm{~cm}^{2} / \mathrm{m}^{2}$ was deemed to be sarcopenia. ${ }^{2}$

\section{Image acquisition}

All CT imaging was routinely performed at our hospital. Patients underwent contrast-enhanced CT scanning of the head and neck area on a Philips scanner with 64 detector rows or more (Philips Healthcare, Best, The Netherlands) at our institution. All routine diagnostic CT protocols include thin slices $(<1-\mathrm{mm})$ and reconstruction at $3-5 \mathrm{~mm}$.

\section{Image evaluation}

Images were typically analyzed in multiple directions by one reader (P.A.d.J), a radiologist with $>10$ years of experience in $\mathrm{CT}$ evaluation and a specific research interest in arterial calcification. The reader was blinded for patient and treatment related factors, as well as for study outcomes (e.g. formation of PCF).

A visual grading system was used similar to previous studies in order to consistently score CT images on arterial calcification at 10 different anatomica locations. ${ }^{18,22}$ The selected locations include large aortic structures (ascending orta, aortic arch, descending aorta and origins of the brachiocephalic arteries), carotid structures (left and right extracranial carotid artery, left and right carotid siphon), and left and right vertebral arteries. Scores of 0, 1,2 and 3 were assigned for all locations except the origins of the brachiocephalic arteries corresponding with the absence of calcifications (score 0 ), mild calcification defined as one or wo dots of calcium smaller than $1 \mathrm{~cm}$ (score 1), moderate calcification defined as one calcification larger than $1 \mathrm{~cm}$ (score 2), and severe calcification defined as circular calcification or a large calcification combined with smaller dots or $>2$ dots (score 3), respectively. For the origins of the brachiocephalic arteries, a score of 0 corresponds with no calcification present, a score of 1 with the calcification of one origin of a brachiocephalic artery, a score of 2 with the calcification of two brachiocephalic arteries and a score of 3 with the calcification of all three brachiocephalic arteries. A cumulative calcification score was calculated of arteria calcification scores at all anatomical sites resulting in a score between 0 and 30 fo total arterial calcification. Table 1 shows the distribution of arterial calcification at the selected anatomical locations. Examples of arterial calcification on CT imaging are presented in Figure 1. 
Table 1: Distribution of arterial calcification on preoperative CT images

\begin{tabular}{|c|c|c|c|c|c|c|}
\hline \multirow[t]{3}{*}{ Anatomical location } & \multirow{2}{*}{\multicolumn{4}{|c|}{$\begin{array}{c}\text { Calcification scores } \\
\text { n (\% of total) }\end{array}$}} & \multirow{2}{*}{\multicolumn{2}{|c|}{$\begin{array}{l}\text { Missing } \\
\text { (\% of total) }\end{array}$}} \\
\hline & & & & & & \\
\hline & 0 -Absent & 1-Mild & 2-Moderate & 3-Severe & & \\
\hline Ascending aorta & $78(34.8)$ & $49(21.9)$ & $20(8.9)$ & $30(13.4)$ & & $(21.0)$ \\
\hline Aortic arch & $37(16.5)$ & $28(12.5)$ & $56(25.0)$ & $78(34.8)$ & & (11.2) \\
\hline Descending aorta & $43(19.2)$ & $27(12.1)$ & $27(12.1)$ & $77(34.4)$ & & (22.3) \\
\hline Origins of the brachiocephalic arteries & $31(13.8)$ & 30 (13.4) & $36(16.1)$ & $117(52.2)$ & & $0(4.5)$ \\
\hline Left extracranial carotid artery & $37(16.5)$ & $24(10.7)$ & $46(20.5)$ & $116(51.8)$ & & $(0.4)$ \\
\hline Right extracranial carotid artery & $39(17.4)$ & 30 (13.4) & $45(20.1)$ & $109(48.7)$ & 1 & $(0.4)$ \\
\hline Left vertebral artery & $176(78.6)$ & $18(8.0)$ & $13(5.8)$ & $13(5.8)$ & 4 & $(1.8)$ \\
\hline Right vertebral artery & $181(80.8)$ & $17(7.6)$ & $13(5.8)$ & $9(4.0)$ & 4 & $(1.8)$ \\
\hline Left carotid siphon & $33(14.7)$ & $31(13.8)$ & $46(20.5)$ & $104(46.4)$ & & $0(4.5)$ \\
\hline Right carotid siphon & $35(15.6)$ & $32(14.3)$ & $40(17.9)$ & $107(47.6)$ & & $0(4.5)$ \\
\hline
\end{tabular}

Figure 1. Examples of preoperative CT images of arterial calcification in patients planned for laryngectomy. A white arrow indicates a moderate to severe calcification (score $\mathbf{2}$ or 3) whereas a black arrow indicates minor calcification (score 1).
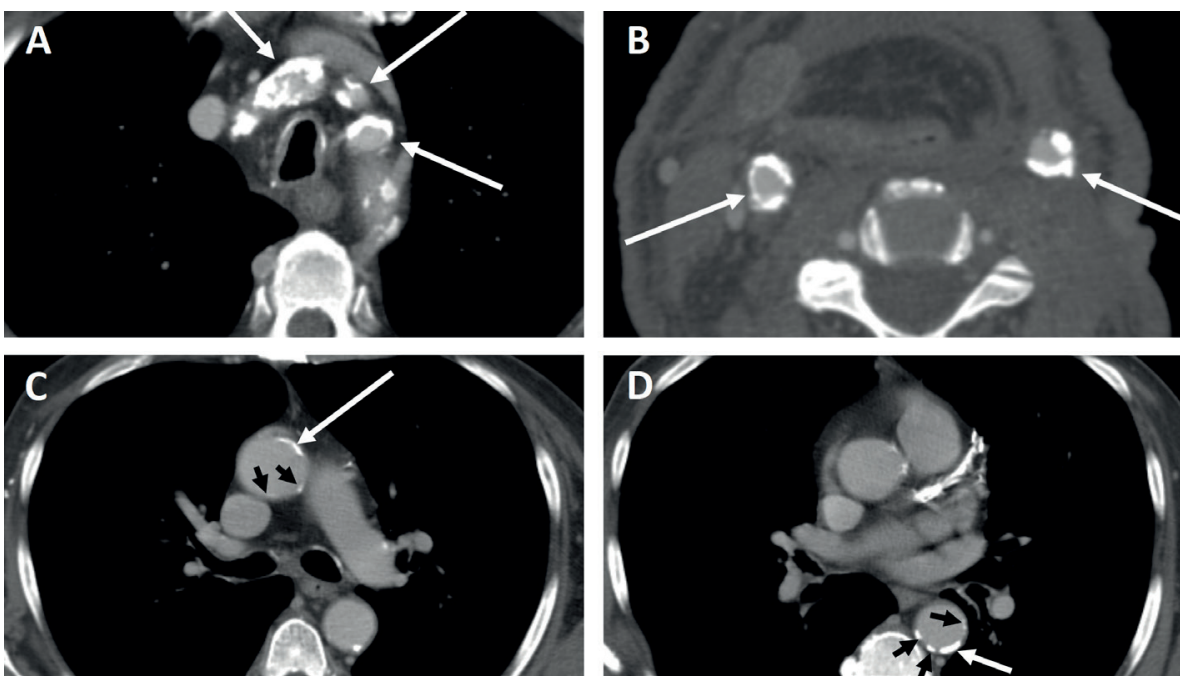

A: Calcification of the origins of all three brachiocephalic arteries, resulting in a score of 3 for calcification of the origins of the brachiocephalic arteries.

B: Calcification of the left and right external carotid arteries, resulting in a score of 3 for calcification of the external carotid arteries.

C: Calcification of the ascending aorta with two minor specs of calcification and one severe calcification, resulting in a score of 2 for calcification of the ascending aorta.

D: Multiple calcified foci of the descending aorta with several minor specs of calcification and one larger segment, resulting in a score of 2 for calcification of the descending aorta.

\section{Statistical analysis}

Categorical data are represented as a number and percentage of the total. A test for normality (Kolmogorov-Smirnoff test) was used to assess whether continuous variables were normally distributed. Continuous data are represented as mean \pm SD if normally distributed, and median \pm interquartile range if skewed. Fisher's exact tests, Pearson Chi square tests, independent sample t tests, and Mann-Whitney $\mathrm{U}$ tests were used to assess group differences where appropriate. Univariable and multivariable backward stepwise logistic regression analysis was used to examine the association between calcifications and PCF. Covariates in regression analysis were chosen based on known or expected association with PCF. Not all anatomical locations could be assessed in all patients, most commonly when CT imaging of the thoracic area was not available or because of dental artefacts. The missing data were considered missing at random. Multiple imputation of these missing sites was applied to replace the missing values for logistic regression analysis, using the median of 20 imputated datasets. ${ }^{23,24}$ Statistical analyses was performed using the IBM SPSS Statistics version 25.0 software package (Chicago, Illinois, USA). All analyses were two-sided and $p \leq 0.05$ was considered significant.

\section{Results}

Between January 2008 and June 2017, 245 patients underwent TL at our institution. Of these 245, 17 patients were excluded because there was no CT imaging available, and 4 patients were excluded because of inadequate quality of imaging. Therefore, 224 patients were included in this analysis. Median interval between imaging and TL was 27 days.

\section{Patient and treatment characteristics}

The 224 patients that were included for analysis had a mean age of 64.8 years. Patients were predominantly male (82.1\%). During the study period, 105 patients 46.9\%) underwent primary TL, 108 patients (48.2\%) underwent salvage TL, and 11 patients (4.9\%) underwent a functional TL. Prior to total laryngectomy, 98 patients (43.8\%) had undergone radiotherapy and 21 patients (9.4\%) had undergone chemoradiotherapy.

A PCF occurred in 62 patients (27.7\%), which required surgical closure in 40 patients (64.5\% of all PCF). Patient, disease and treatment related characteristics, and their relationship with the occurrence of a PCF are presented in Table 2. To summarize, patients who had a PCF more often had hypopharyngeal cancer, a dysfunctional 
Table 2: Patient, disease and treatment characteristics

\begin{tabular}{|c|c|c|c|c|c|}
\hline \multirow{2}{*}{$\begin{array}{l}\text { Characteristic } \\
\text { Sex (male) }\end{array}$} & \multicolumn{2}{|c|}{$\begin{array}{c}\text { With } \\
\text { pharyngocutaneous fistula } \\
\mathrm{n}=62 \text { (\% of total) }\end{array}$} & \multicolumn{2}{|c|}{$\begin{array}{c}\text { Without } \\
\text { pharyngocutaneous fistula } \\
\mathrm{n}=162 \text { (\% of total) }\end{array}$} & \multirow{2}{*}{\begin{tabular}{|c|} 
Pvalue \\
$0.25^{\mathrm{a}}$
\end{tabular}} \\
\hline & 48 & $(77.4)$ & 136 & $(84.0)$ & \\
\hline Age at diagnosis in years (SD) & 64.0 & $(9.2)$ & 65.1 & $(9.1)$ & $0.43^{\mathrm{b}}$ \\
\hline Body mass index (SD) & 23.4 & $(4.8)$ & 24.2 & $(5.1)$ & $0.32^{\mathrm{b}}$ \\
\hline Smoking (current) & 30 & (48.4) & 82 & $\begin{array}{l}(50.6) \\
\end{array}$ & $0.33^{a}$ \\
\hline \multicolumn{6}{|l|}{ Alcohol abuse $^{d}$} \\
\hline Never & 37 & $(59.7)$ & 111 & (68.5) & $0.44 c$ \\
\hline Former & 17 & (27.4) & 33 & (20.4) & \\
\hline Current & 8 & (12.9) & 18 & (11.1) & \\
\hline \multicolumn{6}{|l|}{ ASA classification } \\
\hline 1 & 28 & $(45.2)$ & 80 & (49.4) & 0.84 \\
\hline$\|$ & 18 & $(29.0)$ & 42 & (25.9) & \\
\hline III & 16 & (25.8) & 40 & (24.7) & \\
\hline Presence of sarcopenia & 35 & $(56.5)$ & 67 & $(41.4)$ & $0.04^{\mathrm{a}}$ \\
\hline Localization tumor & & & & & $<0.01^{\mathrm{a}}$ \\
\hline Larynx & 34 & $(54.8)$ & 132 & (81.5) & \\
\hline Hypopharynx & 28 & $(45.2)$ & 30 & (18.5) & \\
\hline \multicolumn{6}{|l|}{ AJCC stage } \\
\hline 0 & 7 & (11.3) & 4 & (2.5) & 0.01 \\
\hline I & 3 & $(4.8)$ & 22 & (13.6) & \\
\hline$\|$ & 11 & $(17.7)$ & 21 & $(13.0)$ & \\
\hline III & 6 & $(9.7)$ & 28 & (17.3) & \\
\hline IV & 35 & $(56.5)$ & 87 & (53.7) & \\
\hline \multicolumn{6}{|l|}{ Indication for TL } \\
\hline Primary HNC & 26 & $(41.9)$ & 79 & (48.8) & $0.02^{c}$ \\
\hline Recurrent HNC & 29 & $(46.7)$ & 79 & (48.8) & \\
\hline Dysfunctional larynx & 7 & $(11.3)$ & 4 & (2.5) & \\
\hline \multicolumn{6}{|l|}{ Prior treatment } \\
\hline None & 26 & $(41.9)$ & 79 & $(48.8)$ & $0.62^{c}$ \\
\hline Radiotherapy & 29 & $(46.8)$ & 69 & (42.6) & \\
\hline Chemo-radiotherapy & 7 & (11.3) & 14 & $(8.6)$ & \\
\hline \multicolumn{6}{|l|}{ Type resection } \\
\hline Laryngectomy & 31 & $(50.0)$ & 120 & (74.1) & $<0.01^{c}$ \\
\hline $\mathrm{LE}+$ partial & 25 & $(40.3)$ & 28 & (17.3) & \\
\hline $\begin{array}{l}\mathrm{LE}+\text { total } \\
\text { pharyngectomy }\end{array}$ & 6 & (9.7) & 14 & (8.6) & \\
\hline \multicolumn{6}{|l|}{ Closure of neopharynx } \\
\hline Vertical & 28 & $(45.2)$ & 110 & (67.9) & $<0.01 c$ \\
\hline T-closure & 5 & $(8.1)$ & 13 & $(8.0)$ & \\
\hline Flap closure & 29 & $(46.8)$ & 39 & (24.1) & \\
\hline \multicolumn{6}{|l|}{ Lymph node dissection } \\
\hline None & 22 & $(35.5)$ & 73 & (45.1) & $0.25^{c}$ \\
\hline Unilateral & 28 & $(45.2)$ & 54 & (33.3) & \\
\hline Bilateral & 12 & $(19.4)$ & 35 & (21.6) & \\
\hline
\end{tabular}

Fisher's exact test. ${ }^{b}$ Independent sample $t$ test. c Pearson Chi squared test. ${ }^{d}$ Defined as the consumption of 5 of more units per day for men and 4 or more units per day for women; or a medical history note including alcohol abuse. larynx after treatment, sarcopenia, laryngectomy with pharyngectomy and flap closure of the neopharynx. Additional lymph node dissection and prior treatment for head and neck cancer not resulting in laryngeal dysfunction was not more common in patients with a PCF. Patients with a PCF were not significantly older and did not have a significantly lower BMI.

\section{Arterial calcification and univariable analysis}

Distribution of arterial calcifications is shown in Table 1. In the majority of patients, presence of arterial calcifications was found. Arterial calcifications of the aortic artery and carotid branches were a common finding. In contrast, arterial calcifications in the vertebral arteries were rare. Only 3 patients $(1.3 \%)$ had no arterial calcification. In 16 patients ( $7.1 \%$ of total), at most mild calcifications were seen. Of those 16 patients, only one patient had a PCF (Pearson Chi square test $p=0.05$ ). For subsequent analysis calcification scores were divided in two groups; none to mild calcifications, and moderate to severe calcifications.

Distribution of arterial calcification among patients with and without PCF and univariable odds ratios are shown in Table 3. Arterial calcifications in all anatomica locations apart from the vertebral arteries were more frequently observed in patients who had a PCF, which is shown in Table 3. Arterial calcification of the descending aorta (OR 2.32, 95\% Cl $1.25-4.29, \mathrm{p}=0.01)$, originges of the brachiocephalic arteries (OR 2.14,95\% Cl $1.05-4.35, \mathrm{p}=0.04$ ), right extracrania carotid artery (OR 2.05, 95\% Cl $1.03-4.09, \mathrm{p}=0.04)$ and left carotid siphon (OR $2.26,95 \% \mathrm{Cl} 1.12-4.59, \mathrm{p}=0.02$ ) were significantly associated with $\mathrm{PC}$ formation. A higher total arterial calcification score was significantly associated with PCF formation (OR 1.06, 95\% Cl $1.01-1.11, \mathrm{p}=0.03)$.

\section{Multivariable logistic regression analysis}

The calcification scores were entered per location into two multivariable logistic regression models, see Table 4 . The first model includes the patient-related variables: age, BMI, sarcopenia, smoking, alcohol abuse, and ASA classification as a surrogate for comorbidities. The second model includes additional known preoperative risk factors for the occurrence of a PCF: localization of tumor (larynx versus hypopharynx), indication for TL (primary, salvage or dysfunctional larynx), additional lymph node dissection, extent of pharyngeal resection and closure method of the neopharynx. 
Table 3: Distribution of arterial calcification in patients with and without

\begin{tabular}{|c|c|c|c|c|c|}
\hline $\begin{array}{l}\text { Anatomical location of arterial } \\
\text { calcification }\end{array}$ & Score ${ }^{a}$ & $\begin{array}{c}\text { With } \\
\text { pharyngocutaneous fistula } \\
\mathrm{n}=62 \text { (\% of total) }\end{array}$ & $\begin{array}{c}\text { Without } \\
\text { pharyngocutaneous fistula } \\
(\mathrm{n}=162) \text { (\% of total) }\end{array}$ & $\begin{array}{l}\text { Unadjusted OR } \\
\quad(95 \% \mathrm{Cl})\end{array}$ & Pvalue \\
\hline Ascending aorta & $\begin{array}{l}0 \\
1\end{array}$ & $\begin{array}{l}31(50.0) \\
31(50.0)\end{array}$ & $\begin{array}{l}101(63.2) \\
61(37.7) \\
\end{array}$ & $\begin{array}{c}1.66 \\
(0.92-2.90)\end{array}$ & 0.09 \\
\hline Aortic arch & & $\begin{array}{l}20(32.3) \\
42(67.7)\end{array}$ & $\begin{array}{l}56(34.6) \\
106(65.4)\end{array}$ & $\begin{array}{c}1.11 \\
(0.60-2.07)\end{array}$ & 0.74 \\
\hline Descending aorta & $\begin{array}{l}0 \\
1 \\
\end{array}$ & $\begin{array}{l}20(32.3) \\
42(67.7) \\
\end{array}$ & $\begin{array}{l}85(52.5) \\
77(47.5) \\
\end{array}$ & $\begin{array}{c}2.32 \\
(1.25-4.29) \\
\end{array}$ & 0.01 \\
\hline $\begin{array}{l}\text { Origins of the brachiocephalic } \\
\text { arteries }\end{array}$ & $\begin{array}{l}0 \\
1 \\
\end{array}$ & $\begin{array}{l}12(19.4) \\
50(80.6) \\
\end{array}$ & $\begin{array}{l}55(34.0) \\
107(66.0) \\
\end{array}$ & $\begin{array}{c}2.14 \\
(1.05-4.35) \\
\end{array}$ & 0.04 \\
\hline Left extracranial carotid artery & $\begin{array}{l}0 \\
1 \\
\end{array}$ & $\begin{array}{l}16(25.8) \\
46(74.2) \\
\end{array}$ & $\begin{array}{l}46(28.4) \\
116(71.6) \\
\end{array}$ & $\begin{array}{c}1.14 \\
(0.59-2.21) \\
\end{array}$ & 0.70 \\
\hline Right extracranial carotid artery & $\begin{array}{l}0 \\
1 \\
\end{array}$ & $\begin{array}{l}13(21.0) \\
49(79.0) \\
\end{array}$ & $\begin{array}{l}57(35.2) \\
105(64.8) \\
\end{array}$ & $\begin{array}{c}2.05 \\
(1.03-4.09)\end{array}$ & 0.04 \\
\hline Left vertebral artery & $\begin{array}{l}0 \\
1 \\
\end{array}$ & $\begin{array}{c}58(93.5) \\
4(6.5) \\
\end{array}$ & $\begin{array}{l}136(84.0) \\
26(16.0) \\
\end{array}$ & $\begin{array}{c}0.36 \\
(0.12-1.08) \\
\end{array}$ & 0.07 \\
\hline Right vertebral artery & $\begin{array}{l}0 \\
1 \\
\end{array}$ & $\begin{array}{c}57(91.9) \\
5(8.1) \\
\end{array}$ & $\begin{array}{l}141(87.0) \\
21(13.0) \\
\end{array}$ & $\begin{array}{c}0.59 \\
(0.21-1.64) \\
\end{array}$ & 0.31 \\
\hline Left carotid siphon & $\begin{array}{l}0 \\
1\end{array}$ & $\begin{array}{l}12(19.4) \\
50(80.6)\end{array}$ & $\begin{array}{l}57(35.2) \\
105(64.8)\end{array}$ & $\begin{array}{c}2.26 \\
(1.12-4.59) \\
\end{array}$ & 0.02 \\
\hline Right carotid siphon & $\begin{array}{l}0 \\
1 \\
\end{array}$ & $\begin{array}{l}15(24.2) \\
47(75.8) \\
\end{array}$ & $\begin{array}{l}60(37.0) \\
102(63.0) \\
\end{array}$ & $\begin{array}{c}1.84 \\
(0.95-3.58) \\
\end{array}$ & 0.07 \\
\hline Total arterial calcification scorec & $\begin{array}{c}\text { Median } \\
\text { IQRd }\end{array}$ & $\begin{array}{c}18.0 \\
12.8-22.0\end{array}$ & $\begin{array}{c}16.0 \\
10.0-21.0\end{array}$ & $\begin{array}{c}1.06 \\
(1.01-1.11)\end{array}$ & 0.03 \\
\hline
\end{tabular}

Numbers in bold: significant at the level of $p \leq 0.05$

Score: 0 - none to mild; 1 - moderate to severe. ${ }^{b}$ Univariable logistic regression analysis.

Continuous; score between 0 and $30 .{ }^{\mathrm{d}}$ Interquartile range

In the first model, arterial calcification of the descending aorta (OR $2.80,95 \% \mathrm{C}$ $1.38-5.66, \mathrm{p}<0.01$ ), origins of the brachiocephalic arteries (OR 2.09, 95\% Cl 1.02 $-4.27, \mathrm{p}=0.04)$, right extracranial carotid artery (OR $2.17,95 \% \mathrm{Cl} 1.08-4.39, \mathrm{p}$ $=0.03$ ) and left carotid siphon (OR 2.83,95\% Cl $1.32-6.08, p=0.01$ ) remained significantly associated with the occurrence of a pharyngocutaneous fistula. In the second model, arterial calcification of the ascending aorta (OR 2.27,95\% Cl 1.16 $4.46, p=0.02)$, descending aorta ( $O R 2.07,95 \% \mathrm{Cl} 1.07-3.99, \mathrm{p}=0.03)$, origins of the brachiocephalic arteries (OR $2.28,95 \% \mathrm{Cl} 1.05-4.92, \mathrm{p}=0.04$ ) and left carotid siphon (OR $2.21,95 \% \mathrm{Cl} 1.04-4.69, \mathrm{p}=0.04$ ) were significantly associated with the occurrence of a pharyngocutaneous fistula. A higher total arterial calcification score was significantly associated with PCF formation in the first (OR 1.08, (\% $\mathrm{Cl} 1.02-1.15, \mathrm{p}=0.01)$ and the second (OR 1.06, 95\% Cl $1.01-1.12, \mathrm{p}=0.03$ ) multivariable model.
Table 4: Multivariable logistic regression analysis for arterial calcification as a risk factor for pharyngocutaneous fistula

\begin{tabular}{|c|c|c|c|c|c|}
\hline $\begin{array}{l}\text { Anatomical location of arterial } \\
\text { calcification }\end{array}$ & Score $\mathrm{a}^{\mathrm{a}}$ & $\begin{array}{c}\text { Adjusted OR 1 }^{\mathrm{b}} \\
(95 \% \mathrm{Cl}) \\
\end{array}$ & $\begin{array}{l}\text { Pvalue } \\
\text { Pval }\end{array}$ & $\begin{array}{c}\text { Adjusted OR 2 }{ }^{c} \\
(95 \% \mathrm{Cl}) \\
\end{array}$ & $\begin{array}{l}\text { Pvalue } \\
\end{array}$ \\
\hline Ascending aorta & $\begin{array}{l}0 \\
1\end{array}$ & $\begin{array}{c}\text { Ref } \\
1.66(0.91-3.02)\end{array}$ & & $\begin{array}{c}\text { Ref } \\
2.27(1.16-4.46)\end{array}$ & 0.02 \\
\hline Aortic arch & $\begin{array}{l}0 \\
1\end{array}$ & $\begin{array}{c}\text { Ref } \\
1.21(0.61-2.41)\end{array}$ & 0.58 & $\begin{array}{c}\text { Ref } \\
1.21(0.61-2.41)\end{array}$ & 0.59 \\
\hline Descending aorta & $\begin{array}{l}0 \\
1\end{array}$ & $\begin{array}{c}\text { Ref } \\
2.80(1.38-5.66)\end{array}$ & $<0.01$ & $\begin{array}{c}\text { Ref } \\
2.07(1.07-3.99)\end{array}$ & 0.03 \\
\hline Origins of the brachiocephalic arteries & $\begin{array}{l}0 \\
1 \\
\end{array}$ & $\begin{array}{c}\text { Ref } \\
2.09(1.02-4.27)\end{array}$ & & $\begin{array}{c}\operatorname{Ref} \\
2.28(1.05-4.92)\end{array}$ & 0.04 \\
\hline Left extracranial carotid artery & $\begin{array}{l}0 \\
1\end{array}$ & $\begin{array}{c}\operatorname{Ref} \\
1.19(0.57-2.48)\end{array}$ & 0.64 & $\begin{array}{c}\operatorname{Ref} \\
0.92(0.45-1.89)\end{array}$ & 0.82 \\
\hline Right extracranial carotid artery & $\begin{array}{l}0 \\
1\end{array}$ & $\begin{array}{c}\text { Ref } \\
2.17(1.08-4.39)\end{array}$ & 0.03 & $\begin{array}{c}\text { Ref } \\
1.84(0.87-3.89)\end{array}$ & 0.11 \\
\hline Left vertebral artery & $\begin{array}{l}0 \\
1\end{array}$ & $\begin{array}{c}\operatorname{Ref} \\
0.33(0.11-1.01)\end{array}$ & 0.05 & $\begin{array}{c}\operatorname{Ref} \\
0.40(0.13-1.25)\end{array}$ & 0.12 \\
\hline Right vertebral artery & $\begin{array}{l}0 \\
1\end{array}$ & $\begin{array}{c}\operatorname{Ref} \\
0.56(0.20-1.59)\end{array}$ & 0.28 & $\begin{array}{c}\operatorname{Ref} \\
0.69(0.23-2.03)\end{array}$ & 0.50 \\
\hline Left carotid siphon & $\begin{array}{l}0 \\
1\end{array}$ & $\begin{array}{c}\text { Ref } \\
2.83(1.32-6.08)\end{array}$ & 0.01 & $\begin{array}{c}\text { Ref } \\
2.21(1.04-4.69)\end{array}$ & 0.04 \\
\hline Right carotid siphon & $\begin{array}{l}0 \\
1 \\
\end{array}$ & $\begin{array}{c}\text { Ref } \\
1.83(0.94-3.57)\end{array}$ & 0.08 & $\begin{array}{c}\text { Ref } \\
1.82(0.90-3.69)\end{array}$ & 0.10 \\
\hline Total arterial calcificat & Cont. ${ }^{d}$ & $1.08(1.02-1.15)$ & 0.01 & $1.06(1.01-1.12)$ & 0.03 \\
\hline
\end{tabular}

Numbers in bold: significant at the level of $p \leq 0.05$

a Score: 0 - none to mild; 1 - moderate to severe

${ }^{\mathrm{b}}$ Multivariate analysis 1: Corrected for: age at diagnosis, BMI, sarcopenia, smoking, alcohol abuse and ASA classification as a surrogate for comorbidities

'Multivariate analysis 2: Corrected for preoperative risk factors: localization of tumor, indication for total laryngectomy (primary, salvage or dysfunctional larynx), additional lymph node dissection extent of pharyngeal resection and closing method of neopharynx

Continuous; score between 0 and 30

\section{Arterial calcification and sarcopenia}

As there may be a shared etiological factor in atherosclerosis and sarcopenia, the occurrence of arterial calcification in patients with and without sarcopenia was explored. Data are shown in Supplementary Data Table 1. Moderate to severe arterial calcification at the location of the descending aorta was significantly more often present in patients with sarcopenia as compared to patients withou sarcopenia (Pearson Chi square test: $p<0.01$ ). At the other locations, no significant difference was observed. The association between arterial calcification and sarcopenia as independent risk factors for PCF formation is shown in Supplementary Data Table 2. In multivariable logistic regression analysis, both 
the total arterial calcification score (adjusted OR 1.05, 95\% CI 1.00-1.10, $\mathrm{p}=0.04$ ) and sarcopenia (adjusted OR 1.86, 95\% Cl $1.02-3.39, \mathrm{p}=0.04$ ) are independently associated with PCF formation.

\section{Discussion}

This retrospective cohort study of patients undergoing laryngectomy shows that arterial calcification is widespread in patients undergoing laryngectomy, and is associated with PCF formation. Moderate to severe arterial calcifications of the descending aorta, origins of the brachiocephalic arteries and left carotid siphon were significantly associated with developing a PCF in univariable and two multivariable regression models. A higher cumulative arterial calcification score (range $0-30$ ) was significantly associated with the occurrence of PCF: the relative risk of PCF increased by 6-8\% per point increase in total arterial calcification score.

Our results are concurrent with recent studies in oesophageal and colorecta surgery. Recent studies in patients undergoing esophagectomy showed that locoregional and generalized cardiovascular disease as identified by visual grading on preoperative imaging was a risk factor for wound healing problems and anastomotic leakage. ${ }^{18,19}$ Another study in patients undergoing colorectal surgery showed that visually graded calcification of the abdominal aorta was associated with increased morbidity after surgery. ${ }^{25}$ It is hypothesized that both locoregional and generalized arterial vascular disease may have a detrimental effect on wound and anastomosis healing due to low flow or hypoperfusion of the surgical area, leading to ischemia. ${ }^{19,26}$

The occurrence of a PCF after TL is a severe complication. It is associated with prolonged hospital stay and feeding tube dependency, as well as decreased quality of life, and it negatively affects survival. Recently, radiologically assessed sarcopenia was identified as a preoperative risk factor for PCF and wound complications in head and neck cancer patients. ${ }^{8,9,27}$ There may be a link between the presence of arterial calcifications and sarcopenia, as systemic inflammation may be a shared etiological factor. The copresence of sarcopenia and arterial calcification was often observed. In multivariable regression analysis, the presence of sarcopenia and arterial calcifications were both independent predictors of PCF.

Routinely performed CT imaging provides additional information on patients functional and biological status, and may aid in the identification of high risk patients for the occurrence of adverse outcomes. Accurate identification of high risk patients for PCF may provide an opportunity for preoperative interventions to decrease the risk. It seems impossible to decrease the amount of arteria calcifications in the preoperative period, but preoperative optimization of general cardiovascular status or other risk factors associated with PCF which coexist might decrease the risk of a PCF. ${ }^{28-30}$ Arterial calcifications as evidence for cardiovascular disease may warrant further examination and intervention prior to surgery. A surgical solution to decrease the risk of PCF in high risk patients may be to use a pectoralis major overlay flap to reinforce the suture line of the neopharynx by covering it with healthy muscle. ${ }^{31}$ In reconstructive microsurgery, radiological evidence of atherosclerosis may also aid in choosing the optimal flap for recontruction. ${ }^{32}$

There are several limitations that need to be addressed. Relevant clinical data such as known cardiovascular disease and diabetes was missing in our database due to missing information in particular in the earlier years of the study period. Also, some traditional cardiovascular risk factors such as serum cholesterol are missing, because these are not routinely measured at our clinic. Smoking and age was included in analysis, and the ASA classification was used as a surrogate for comorbidities, but we acknowledge that this provides limited information on specific comorbidities. ${ }^{33}$ Recent studies suggest that coronary arterial calcification scores or peripheral arterial calcification scores derived from CT imaging are reliable assessment methods for cardiovascular disease, and can identify patients at high risk that would not have been identified using traditional cardiovascula risk factors. ${ }^{14,34,35}$ Second, a visual grading system for arterial calcification as opposed to calcium scores may lead to an observer bias and necessitates a learning curve. Automatic calcium scoring systems are not available using head and neck contrast enhanced $C T$ imaging, but research into automatic arteria calcification scoring on contrast-enhanced CT imaging is ongoing, and this may in the future be available. ${ }^{36,37}$ Machine learning and radiomics using CT features, e.g. skeletal muscle mass, skeletal muscle quality and arterial calcification, from routinely performed head and neck $\mathrm{CT}$ imaging may aid in identifying patients at high risk for fistula formation after laryngectomy in the future. In this study, al calcification scoring was performed by one observer; an experienced radiologist with a research interest in and extensive experience with arterial calcification on $\mathrm{CT}$ imaging. The inter- and intraobserver variability was not researched in this study, but previously found to be good in several studies also in less experienced observers. ${ }^{18,38}$ 
Acknowledging these limitations, we believe that this study provides a relevant novel application of routinely performed, readily available CT imaging for optimization of the identification process of patients undergoing TL at high risk of developing a PCF. More research into the method of quantification of arterial calcification in head and neck cancer patients is warranted and clarification of its relevance for fistula prevention is needed.

\section{Conclusion}

Arterial calcification is widespread in patients undergoing laryngectomy and is associated with pharyngocutaneous fistula formation. Extensive arterial calcification on preoperative $\mathrm{CT}$ imaging may be taken into consideration as a preoperative risk factor for pharyngocutaneous fistula in patients undergoing laryngectomy.

\section{References}

. van der Putten $L$, de Bree R, Kuik DJ, et al. Salvage laryngectomy: Oncological and functiona outcome. Oral Oncol. 2011;47(4):296-301.

2. Theunissen EAR, Timmermans AJ, Zuur CL, et al. Total Laryngectomy for a Dysfunctional Laryn After (Chemo)Radiotherapy. Arch Otolaryngol Neck Surg. 2012;138(6):548.

3. Paydarfar JA, Birkmeyer NJ. Complications in Head and Neck Surgery. Arch Otolaryngol Neck Surg. 2006;132(1):67.

4. Goepfert RP, Hutcheson KA, Lewin JS, et al. Complications, hospital length of stay, and readmission after total laryngectomy. Cancer. 2017;123(10):1760-1767.

5. Hasan Z, Dwivedi RC, Gunaratne DA, Virk SA, Palme CE, Riffat F. Systematic review and metaanalysis of the complications of salvage total laryngectomy. Eur J Surg Oncol. 2017;43(1):42-51. 6. Timmermans AJ, Lansaat L, Theunissen EAR, Hamming-Vrieze O, Hilgers FJM, van den Breke MWM. Predictive Factors for Pharyngocutaneous Fistulization After Total Laryngectomy. Ann Otol Rhinol Laryngol. 2014;123(3):153-161.

7. Lansaat L, van der Noort V, Bernard SE, et al. Predictive factors for pharyngocutaneous fistulization after total laryngectomy: a Dutch Head and Neck Society audit. Eur Arch OtoRhino-Laryngology. 2018;275(3):783-794

8. Bril SI, Pezier TF, Tijink BM, Janssen LM, Braunius WW, Bree R. Preoperative low skeletal muscle mass as a risk factor for pharyngocutaneous fistula and decreased overall survival in patient undergoing total laryngectomy. Head Neck. 2019;41(6):1745-1755.

9. Achim V, Bash J, Mowery A, et al. Prognostic Indication of Sarcopenia for Wound Complication After Total Laryngectomy. JAMA Otolaryngol Neck Surg. 2017;143(12):1159.

10. Thawani R, McLane M, Beig N, et al. Radiomics and radiogenomics in lung cancer: A review for the clinician. Lung Cancer. 2018;115:34-41.

11. Weston AD, Korfiatis P, Kline TL, et al. Automated Abdominal Segmentation of CT Scans for Body Composition Analysis Using Deep Learning. Radiology. 2019;290(3):669-679.

12. Swartz JE, Pothen AJ, Wegner I, et al. Feasibility of using head and neck CT imaging to assess skeletal muscle mass in head and neck cancer patients. Oral Oncol. 2016;62:28-33.

13. Gondrie MJA, Mali WPTM, Jacobs PC, Oen AL, van der Graaf Y. Cardiovascular Disease: Prediction with Ancillary Aortic Findings on Chest CT Scans in Routine Practice. Radiology. 2010;257(2):549-559.

14. Gernaat SAM, Is̆gum I, de Vos BD, et al. Automatic Coronary Artery Calcium Scoring on Radiotherapy Planning CT Scans of Breast Cancer Patients: Reproducibility and Association with Traditional Cardiovascular Risk Factors. Zirlik A, ed. PLoS One. 2016;11(12):e0167925.

15. Siasos G, Tsigkou V, Kokkou E, et al. Smoking and Atherosclerosis: Mechanisms of Disease and New Therapeutic Approaches. Curr Med Chem. 2014;21(34):3936-3948.

16. Hansson GK. Inflammation, Atherosclerosis, and Coronary Artery Disease. N Engl J Med. 2005;352(16):1685-1695.

17. Dalle S, Rossmeislova L, Koppo K. The Role of Inflammation in Age-Related Sarcopenia. Front Physiol. 2017;8.

18. Borggreve AS, Goense L, van Rossum PSN, van Hillegersberg R, de Jong PA, Ruurda JP. Generalized cardiovascular disease on a preoperative CT scan is predictive for anastomotic leakage after esophagectomy. Eur J Surg Oncol. 2018;44(5):587-593.

19. Goense L, van Rossum PSN, Weijs TJ, et al. Aortic Calcification Increases the Risk of Anastomotic Leakage After Ivor-Lewis Esophagectomy. Ann Thorac Surg. 2016;102(1):247-252.

20. Pochhammer $J$, Tröster $F$, Blumenstock $G$, et al. Calcification of the iliac arteries: a marker for leakage risk in rectal anastomosis—a blinded clinical trial. Int J Colorectal Dis. 2018;33(2):163170.

21. Wendrich AW, Swartz JE, Bril SI, et al. Low skeletal muscle mass is a predictive factor for chemotherapy dose-limiting toxicity in patients with locally advanced head and neck cancer. Oral Oncol. 2017;71:26-33. 
22. Jairam PM, de Jong PA, Mali WPTM, Isgum I, van der Graaf Y. Cardiovascular disease prediction do pulmonary disease-related chest CT features have added value? Eur Radiol. 2015;25(6):16461654.

23. Sterne JAC, White IR, Carlin JB, et al. Multiple imputation for missing data in epidemiological and clinical research: potential and pitfalls. BMJ. 2009;338(jun29 1):b2393-b2393.

24. Siew ED, Peterson JF, Eden SK, Moons KG, Ikizler TA, Matheny ME. Use of Multiple Imputation Method to Improve Estimation of Missing Baseline Serum Creatinine in Acute Kidney Injury Research. Clin J Am Soc Nephrol. 2013;8(1):10-18.

25. Eveno C, Latrasse V, Gayat É, Lo Dico R, Dohan A, Pocard M. Colorectal anastomotic leakage can be predicted by abdominal aortic calcification on preoperative CT scans: A pilot study. J Visc (Pug, $2016,153(4): 253-257$

26. Pham TH, Perry KA, Enestvedt CK, et al. Decreased Conduit Perfusion Measured by Spectroscopy Is Associated With Anastomotic Complications. Ann Thorac Surg. 2011;91(2):380-385.

7. Ansari E, Chargi N, van Gemert JTM, et al. Low skeletal muscle mass is a strong predictive factor for surgical complications and a prognostic factor in oral cancer patients undergoin mandibular reconstruction with a free fibula flap.

28. Tew GA, Ayyash R, Durrand J, Danjoux GR. Clinical guideline and recommendations on preoperative exercise training in patients awaiting major non-cardiac surgery. Anaesthesia. 2018;73(6):750-768

29. Huang J, Lai Y, Zhou X, et al. Short-term high-intensity rehabilitation in radically treated lung cancer: a three-armed randomized controlled trial. J Thorac Dis. 2017;9(7):1919-1929.

30. Minnella EM, Awasthi R, Loiselle S-E, Agnihotram R V., Ferri LE, Carli F. Effect of Exercise and Nutrition Prehabilitation on Functional Capacity in Esophagogastric Cancer Surgery. JAMA Surg. 2018;153(12):1081.

31. Gil Z, Gupta A, Kummer B, et al. The Role of Pectoralis Major Muscle Flap in Salvage Tota Laryngectomy. Arch Otolaryngol Neck Surg. 2009;135(10):1019.

32. Bouaoud J, Honart J-F, Bennis Y, Leymarie N. How to manage calcified vessels for head and neck microsurgical reconstruction. J Stomatol Oral Maxillofac Surg. 2020;121(4):439-441.

33. Hurwitz EE, Simon M, Vinta SR, et al. Adding Examples to the ASA-Physical Status Classification Improves Correct Assignment to Patients. Anesthesiology. 2017;126(4):614-622.

34. Tatami $Y$, Yasuda $Y$, Suzuki $S$, et al. Impact of abdominal aortic calcification on longterm cardiovascular outcomes in patients with chronic kidney disease. Atherosclerosis. 2015;243(2):349-355.

35. Peeters MJ, van den Brand JA, van Zuilen AD, et al. Abdominal aortic calcification in patients with CKD. J Nephrol. 2017;30(1):109-118. doi:10.1007/s40620-015-0260-7

36. van Velzen SGM, Lessmann N, Velthuis BK, et al. Deep Learning for Automatic Calcium Scoring in CT:Validation Using Multiple Cardiac CT and Chest CT Protocols. Radiology. 2020;295(1):66-

37. Isgum I, Prokop M, Niemeijer M, Viergever MA, van Ginneken B. Automatic Coronary Calcium Scoring in Low-Dose Chest Computed Tomography. IEEE Trans Med Imaging. 2012;31(12):23222334.

38. Jacobs PCA, Prokop M, Oen AL, van der Graaf Y, Grobbee DE, Mali WPTM. Semiquantitative Assessment of Cardiovascular Disease Markers in Multislice Computed Tomography of the Chest. J Comput Assist Tomogr. 2010;34(2):279-284.

\section{Supplementary Data}

Table 1: presence of arterial calcification and sarcopenia

\begin{tabular}{|c|c|c|c|c|}
\hline Anatomical location of arterial calcification & Score ${ }^{a}$ & $\begin{array}{c}\text { Sarcopenia } \\
(\mathrm{n}=103)\end{array}$ & $\begin{array}{c}\text { Normal skeletal muscle mass } \\
(n=121)\end{array}$ & Pvalue \\
\hline Ascending aorta & $\begin{array}{l}0 \\
1\end{array}$ & $\begin{array}{l}60 \\
43\end{array}$ & $\begin{array}{l}72 \\
49\end{array}$ & $0.85^{b}$ \\
\hline Aortic arch & $\begin{array}{l}0 \\
1\end{array}$ & $\begin{array}{l}32 \\
71\end{array}$ & $\begin{array}{l}44 \\
77\end{array}$ & $0.40^{\mathrm{b}}$ \\
\hline Descending aorta & $\begin{array}{l}0 \\
1\end{array}$ & $\begin{array}{l}36 \\
67\end{array}$ & $\begin{array}{l}69 \\
52\end{array}$ & $<0.01^{\natural}$ \\
\hline Origins of the brachiocephalic arteries & $\begin{array}{l}0 \\
1\end{array}$ & $\begin{array}{l}28 \\
75\end{array}$ & $\begin{array}{l}39 \\
82\end{array}$ & $0.41^{b}$ \\
\hline Left extracranial carotid artery & $\begin{array}{l}0 \\
1\end{array}$ & $\begin{array}{l}28 \\
75\end{array}$ & $\begin{array}{l}34 \\
87\end{array}$ & $0.88^{b}$ \\
\hline Right extracranial carotid artery & $\begin{array}{l}0 \\
1\end{array}$ & $\begin{array}{l}35 \\
68\end{array}$ & $\begin{array}{l}35 \\
86\end{array}$ & $0.42^{b}$ \\
\hline Left vertebral artery & $\begin{array}{l}0 \\
1\end{array}$ & $\begin{array}{l}87 \\
16\end{array}$ & $\begin{array}{l}107 \\
14\end{array}$ & $0.39^{b}$ \\
\hline Right vertebral artery & $\begin{array}{l}0 \\
1\end{array}$ & $\begin{array}{l}90 \\
13\end{array}$ & $\begin{array}{l}108 \\
13\end{array}$ & $0.66^{b}$ \\
\hline Left carotid siphon & $\begin{array}{l}0 \\
1\end{array}$ & $\begin{array}{l}30 \\
73\end{array}$ & $\begin{array}{l}39 \\
82\end{array}$ & $0.62^{b}$ \\
\hline Right carotid siphon & $\begin{array}{l}0 \\
1\end{array}$ & $\begin{array}{l}33 \\
70\end{array}$ & $\begin{array}{l}42 \\
79\end{array}$ & $0.67^{b}$ \\
\hline Total arterial calcification score ${ }^{d}$ & $\begin{array}{c}\text { Mean } \\
\text { SD }\end{array}$ & $\begin{array}{c}16.5 \\
7.4\end{array}$ & $\begin{array}{c}15.1 \\
6.8\end{array}$ & $0.09 c$ \\
\hline
\end{tabular}

Numbers in bold: significant at the level of $p \leq 0.05$

a Score: 0 - none to mild; 1 - moderate to severe

bPearson Chi square test

'Mann-Whitney U test

Continuous; score between 0 and 30

Table 2: Sarcopenia and arterial calcification as predictors of pharyngocutaneous

fistula

\begin{tabular}{llllll}
\hline & Value & Unadjusted $0 R^{\mathrm{b}}(95 \% \mathrm{Cl})$ & P value & Adjusted $0 \mathrm{R}^{\mathrm{c}}(\mathbf{9 5} \% \mathrm{Cl})$ & P value \\
\hline $\begin{array}{l}\text { Total arterial } \\
\text { calcification score }\end{array}$ & Cont. & $1.06(1.01-1.11)$ & 0.03 & $1.05(1.00-1.10)$ & 0.04 \\
Sarcopenia & & & & & \\
& No & Ref & 0.03 & $1.86(1.02-3.39)$ & 0.04 \\
\hline
\end{tabular}

Numbers in bold: significant at the level of $p \leq 0.05$

a Continuous; score between 0 and 30

Univariable regression analysis

Multivariable regression analysis using a backward stepwise selection 


\section{Part IV}

Future perspectives 


\section{Chapter 10}

A study protocol for an observational study investigating the importance of skeletal muscle mass for cisplatin pharmacokinetics in patients with head and neck cancer: the PLATISMA study

Sandra I. Bril, Najiba C. Chargi, Geert W.J. Frederix, Lot A. Devriese, Alwin D.R

Huitema, Remco de Bree

Summarized from KWF grant submission 2017-II 


\section{Abstract}

Background Treatment of advanced stage head and neck squamous cell carcinoma (HNSCC) with chemoradiotherapy with cisplatin is associated with severe toxicity, requiring treatment de-escalation or termination of chemotherapy (dose-limiting toxicity) in at least $30 \%$ of patients. Chemotherapy dose limiting toxicity negatively affects the survival of patients. In recent years, a relationship between low skeletal muscle mass (SMM) and toxicity has been described, which may be related to altered cisplatin distribution and binding into the fat-free body mass, of which SMM is the largest contributor. Nevertheless, most cytotoxic agents, including cisplatin, are dosed based on body surface area (BSA), which does not take individual body composition into account. Data on the relationship between body composition and pharmacokinetic characteristics of cisplatin is not available. This study aims to investigate the association between cisplatin pharmacokinetics, SMM and BSA in HNSCC patients treated with chemoradiotherapy with cisplatin.

Study design This study is designed as a prospective observational study in HNSCC patients treated with chemoradiotherapy with cisplatin. All patients will receive standard-of-care BSA based chemoradiotherapy with three cycles of cisplatin, at a dose level of $100 \mathrm{mg} / \mathrm{m} 2$ per cycle. Quantitative data on SMM and cisplatin pharmacokinetics (total and unbound plasma concentration) will be collected, as well as data on toxicity and quality of life. Cisplatin related toxicity will be scored as treatment de-escalation or termination, and as clinically relevant toxicity. The primary outcome of this study is the relationship between cisplatin pharmacokinetics, SMM and BSA. Secondary outcomes are cisplatin related toxicity and dose limiting toxicity, quality of life, hospital costs and biomarkers associated with low SMM.

Discussion This observational study will provide evidence for the relationship between body composition and cisplatin pharmacokinetics in HNSCC patients. Cisplatin dosing based on individual body composition instead of body surface area may result in less toxicity while maintaining efficacy and may contribute to precision medicine. If the hypothesis of this study is correct, the next step will be to test a novel cisplatin dosing scheme based on body composition in a randomized controlled trial. The results of this study may also be used as a proofof-principle in research in other types of cancer and cytotoxic drugs.

\section{Background}

Head and neck cancers (HNCs) are among the most frequent tumors in the world with an estimated 835.000 new cases and 428.000 deaths in 2018. ${ }^{1}$ There has been a significant increase in the global incidence of HNC over the past decade. ${ }^{1,2}$ In the Netherlands, 3000 patients are diagnosed with HNC yearly, and the incidence of HNC has increased from 16.35 in 2005 to 18.1 per 100,000 person-years in 2015. ${ }^{3}$ More than $90 \%$ of all HNCs are head and neck squamous cell carcinomas (HNSCC). ${ }^{4}$ Prolonged tobacco use and alcohol consumption are the main risk factors associated with HNSCC.5,6 In increasing numbers, a specific subset of HNSCC located in the oropharynx is caused by human papillomaviruses (HPV), most specifically HPV 16 and 18.,8 Two-thirds of HNSCC patients present with advanced disease at diagnosis (large tumor, regional metastasis, and/or distant metastasis). Distant metastasis at diagnosis is uncommon, so the treatment of advanced stage HNSCC is most often with curative intent. ${ }^{5}$ Standard treatment of advanced stage HNSCC is chemoradiotherapy (CRT). Commonly, CRT with curative intent consists of radiotherapy with 66 to 70 Gray total radiation dose, combined with 3 cycles high dose cisplatin at $100 \mathrm{mg} / \mathrm{m}^{2}$ body surface area per cycle. ${ }^{10}$

Due to the addition of cisplatin to primary radiation treatment in HNSCC, disease specific survival has improved with approximately $8 \% .{ }^{11}$ Primary CRT also offers the opportunity of organ preservation (e.g. larynx) in advanced stage tumors with roughly similar survival as primary surgery. ${ }^{12}$ However, the addition of cisplatin to radiation treatment can cause severe side effects, such as nausea, nephrotoxicity, bone marrow depression and ototoxicity. ${ }^{13,14}$ It is currently difficult to predict which patients are at risk of developing severe cisplatin related toxicity. Acute toxicity is a common and serious problem in HNSCC patients, and results in dose-reductions, treatment delay or treatment termination (chemotherapy dose limiting toxicity, CDLT) in at least $30 \%$ of the patients. ${ }^{13,15,16}$

It can be anticipated that CDLT has a negative effect on the survival of HNSCC patients, because patients receive a suboptimal treatment. A retrospective cohort study has shown that a cumulative cisplatin dose of less than $200 \mathrm{mg} / \mathrm{m}^{2}$ is associated with a significantly lower overall survival in HNSCC patients treated with CRT. ${ }^{15}$ Treatment-related toxicity itself can also have a negative effect on short-term and long-term survival. ${ }^{17}$ The occurrence of significant toxicity may also have a negative effect on the quality of life of patients and may result in higher healthcare related costs due to, for instance, readmission to the hospital. 
In the last decade, the body composition of cancer patients has been investigated extensively using standard diagnostic CT imaging. ${ }^{18}$ The different body compartments, such as skeletal muscle mass (SMM) and adipose tissue mass, can be accurately quantified using this method. ${ }^{19,20}$ Specifically, a low skeletal muscle mass, sometimes termed sarcopenia, has been related to negative outcomes in variety of tumor types and treatments. ${ }^{21-23}$ Risk factors for low SMM are, amongst others, old age, malnutrition, immobility and illness; factors which are common in HNSCC patients. ${ }^{24,25}$

In recent years a relationship between radiologically assessed low SMM and chemotherapy related toxicity has been described in a variety of tumor types, including $\mathrm{HNSCC} .{ }^{23,26,27}$ A recent retrospective cohort study in patients with $\mathrm{HNSCC}$ undergoing primary CRT with cisplatin showed that patients with low SMM had a 3 -fold higher risk of experiencing CDLT (44.3\% vs. $13.7 \%){ }^{26}$ Patients with low SMM received a significantly higher cisplatin dose per kilogram of estimated total lean body mass than patients with normal skeletal muscle mass $(4.6 \mathrm{mg} / \mathrm{kg}$ versus $3.8 \mathrm{mg} / \mathrm{kg}$ estimated lean body mass). Patients who had experienced CDLT had a significantly shorter overall survival than patients who were able to complete CRT. ${ }^{26}$ The results in HNSCC are in accordance with results of retrospective cohort studies in other types of cancer and with other types of chemotherapy. ${ }^{28}$

Patients most at risk of chemotherapy related toxicity appear to be those with both low SMM and obesity, a phenomenon sometimes termed sarcopenic obesity. ${ }^{29}$ An explanation for this relationship is that hydrophilic cytotoxic drugs, including cisplatin, mainly distribute into the fat-free body mass. Skeletal muscle mass is the largest contributor to the fat-free body mass. ${ }^{30}$ Cisplatin dose is currently calculated using an estimated body surface area (BSA), which does not take into account the individual body composition. It has been described previously that BSA is a poor predictor of systemic exposure to anticancer drugs, including cisplatin, but a better alternative is not available yet. ${ }^{31-33} \mathrm{~A}$ possible explanation for this phenomenon may be that patients with a disproportionally low fat-free mass and high fat mass may actually receive a relative overdose when chemotherapy is dosed based on BSA due to the distributional properties of the drug and the individual body composition of the patient. Also, cisplatin binds irreversibly to proteins and water-containing tissue. The reservoir of protein and water-containing tissue is lower in patients with low SMM and normal or high fat mass compared to patients with normal SMM, which may contribute to the increased incidence of toxicity. ${ }^{34,35}$
Thus, HNSCC patients with low SMM and normal or high body weight may actually receive a relative overdose of cisplatin when the cisplatin dose is calculated using BSA. Direct evidence to prove this hypothesis is currently not available because pharmacokinetic (PK) parameters of cisplatin and SMM are not routinely measured in HNSCC patients. In other types of cancer and anticancer drugs, prospective research to prove this hypothesis is not available either. ${ }^{28}$

The primary aim of this prospective study is to investigate the relationship between SMM and PK parameters of cisplatin (total and free plasma concentrations) in HNSCC patients treated with conventional CRT with cisplatin. The hypothesis is that cisplatin PK is better associated with SMM than with BSA, which would explain why patients with low SMM are more prone to experience cisplatin related toxicity. Possibly, the current cisplatin dosing method using BSA insufficiently takes into account individual differences in body composition, which would result in a relative overdose in cisplatin in patients with low SMM. The results of this study may be used in a future prospective randomized controlled trial investigating an adapted dosing scheme for cisplatin based on the individual body composition of a patient. This may lead to personalized cisplatin dosing in HNSCC patients, with less acute toxicity and improved efficacy of treatment.

\section{Methods}

\section{Design}

This study is designed as a monocenter prospective observational cohort study in HNSCC patients receiving primary CRT. The study is expected to include 50 patients in approximately 1.5 years at our tertiary referral center specialized in head and neck cancer treatment. The Medical Research Ethics Committee (METC) of the University Medical Center Utrecht has reviewed the study in accordance with the Dutch Medical Research Involving Human Subjects Act (WMO) and other applicable Dutch and European regulations, and has issued and approval of this study in June 2018 (METC 18-225/D). Yearly, approximately 50 patients with HNSCC are treated with CRT at our center. HNSCC patients who are scheduled for chemoradiotherapy with cisplatin will be recruited by their head and neck surgical oncologist or medical oncologist. Patients will be asked to give informed consent for participation in this study. Patients will be asked to provide blood samples before start of CRT for biomarker assessment and during the first cycle of cisplatin for pharmacokinetics. Patients will be asked to complete quality of life questionnaires before start of CRT, after the first cisplatin cycle, and after the end 


\section{Inclusion- and exclusion criteria}

In order to be eligible to participate in this study, a subject must meet all of the following criteria:

- Diagnosed with HNSCC (histologically or cytology proven).

- Scheduled for CRT with high dose cisplatin.

- Eighteen years of age or older, and able to exercise their free will.

- Sufficient understanding of the Dutch language to give informed consent.

A potential subject who meets any of the following criteria will be excluded from participation in this study if the patient has:

- Major CT artefacts, impeding accurate muscle identification on CT imaging.

- Synchronous tumor(s) outside of the head and neck region, e.g. concurrent non-small cell lung cancer, for which the patient will receive concurrent treatment.

\section{Aims of this study}

The primary aim of this study is to investigate the relationship between PK parameters of cisplatin, SMM and BSA. A secondary aim of this study is to investigate the relationship between PK parameters of cisplatin and cisplatin related toxicity. Explorative aims of this study are: to investigate the relationship between low SMM and quality of life; between cisplatin related toxicity and quality of life; to investigate the healthcare related costs of patients undergoing CRT and its relationship with low SMM; and to investigate the relationship between low SMM and biomarkers for systemic inflammation and cancer cachexia in patients with HNSCC undergoing CRT.

\section{Clinical characteristics}

Patient characteristics will be recorded at diagnosis, start of treatment and during treatment according to standardized protocols. Amongst others, gender, age, ethnicity, height, body weight, tumor type, tumor site, TNM classification, HPV status, smoking history, alcohol intake, weight loss, comorbidities and renal function will be recorded.

\section{Skeletal muscle mass}

Skeletal muscle mass will be measured on pre-treatment CT imaging of the head and neck area at the level of $\mathrm{C} 3$, and on pre-treatment CT imaging of the abdominal area at the level of L3. For SMM measurement at the level of C3, the first slide when scrolling from caudal to cranial direction to show both transverse processes and the entire vertebral arc will be selected for segmentation of muscle tissue. ${ }^{36}$ The paravertebral muscles (PVM) and both sternocleidomastoid (SCM) muscles will be manually segmented. For SMM measurement at the level of L3, the first CT slide when scrolling from caudal to cranial direction showing both transverse processes and the spinous process will be selected for segmentation. The psoas muscles, paraspinal muscles and muscles of the abdominal wall will be manually segmented. ${ }^{20}$ Muscle area will be defined as the segmented pixel area on the CT slide with a radiodensity between -29 and +150 Hounsfield Unit (HU), which is specific for muscle tissue..$^{37}$ Segmentation of muscle tissue will be manually performed using the commercially available software package SliceOmatic (Tomovision, Canada).

\section{Bioanalysis and pharmacokinetics of cisplatin}

Pharmacokinetic parameters of cisplatin will be measured in plasma samples collected from patients at different time point (pre-dose, end of cisplatin infusion and 1 hour, 3 hours, 7 hours and 24 hours after start of infusion) during the first cycle of cisplatin. Both total plasma and free plasma concentrations of cisplatin will be measured by inductively couple plasma - mass spectrometry (ICP-MS) by a previously described method. ${ }^{38}$

\section{Treatment related toxicity}

Clinically relevant treatment related toxicity parameters, including specific toxicity that results in significant (grade 3 or 4) toxicity, and any grade toxicity that results in treatment de-escalation or termination will be recorded by the treating medical oncologist. Toxicity will be scored according to the Common Terminology Criteria for Adverse Events (CTCAE) guidelines, version 4. Any toxicity causing unplanned hospital visits or hospital admission will also be recorded.

\section{Quality of life}

Quality of life will be measured by means of the EORTC QLQ-C30, QLQ-H\&N35 and EQ-5D-5L questionnaires and with the USD-head neck, a head and neck specific measurement tool for patient burden used in our hospital to assess patient burden in daily practice. Quality of life measurements are performed at 3 time points: before start of treatment, after the first cycle of cisplatin (when patients return for 
the second cycle of cisplatin), and after the end of CRT, at the final follow up visit at the medical oncologist.

\section{Biomarkers}

Free cisplatin, which is considered the pharmacologically active fraction, is cleared through the renal system, and an impaired renal function may result in decreased cisplatin clearance and increased systemic cisplatin exposure. During standard of care treatment work-up, renal function will be assessed by an estimation of glomerular filtration (eGFR) by measuring creatinine levels in blood. As blood creatinine levels may provide an overestimation of renal function in patients with low SMM, renal function will also be assessed directly by measuring 24-hours creatinine clearance in urine, and by measurement of cystatin $C$ in a pre-treatment blood sample. Cystatin $\mathrm{C}$ is a novel biomarker for renal function with promising results. ${ }^{39,40}$ Biomarkers for poor nutritional status and systemic inflammation, such as low albumin or an increased neutrophil-to-lymphocyte ratio, have been associated with low SMM in medical literature. ${ }^{41,42}$ These biomarkers are not routinely measured in HNSCC patients. In this prospective study the biomarkers will be systematically measured in all patients in order to gain more insight in the nutritional and inflammatory status of the patients. Biomarkers (CRP, BSE, neutrophil-to-lymphocyte ratio, albumin, interleukin 1 (IL-1), interleukin 2 (IL2), interleukin 6 (IL-6), interleukin 10 (IL-10), tumor necrosis factor a (TNF-a) and interferon $\gamma(\mathrm{IFN}-\gamma)$ ) will be measured once in the same pre-treatment blood sample that is taken for cystatin $\mathrm{C}$ measurement.

\section{Healthcare related costs}

Research in patients with gastrointestinal cancers undergoing surgery has shown that low SMM is associated with increased hospital costs. ${ }^{43}$ In this study, the cost analysis will focus on direct medical costs from the hospital's perspective. Costs of treatment and follow-up are based on the total clinical consumption of all evaluable patients including planned and unplanned hospital visits and admissions. The decrease in healthcare related costs and the gains in QoL and QALY's when dosing cisplatin based on skeletal muscle mass will be estimated in a complete economic evaluation in which costs and effects will be linked.

\section{Sample size}

The primary aim of this study is to determine the association between SMM and cisplatin pharmacokinetics, and BSA and cisplatin pharmacokinetics. A clinically representative locally advanced HNSCC patient dataset with BSA values and lean body mass values was simulated using previously published clinical patient characteristics. ${ }^{26,35}$ To estimate the required sample size a, clinical trial simulation $(n=200)$ was performed based on a previously published pharmacokinetics (PK) model on free cisplatin concentrations in plasma. ${ }^{44} \mathrm{An}$ allometric relationship between skeletal muscle mass and cisplatin clearance was assumed. Patient characteristics (SMM and BSA) were simulated to be in accordance to clinical practice. It was estimated that data from 45 patients was sufficient to find a significant relationship between cisplatin pharmacokinetics and SMM with a power of $>80 \%$. As pharmacokinetics models with SMM and BSA are nonhierarchical, the difference between the two models cannot be statistically tested. However, in approximately $70 \%$ of the trials, this relationship showed better goodness-of-fit than a BSA-based relationship. Finally, the allometric exponent could be estimated with acceptable precision (approximately $28 \%$ relative standard error) with a sample size of 45 patients. To account for a drop-out rate of $10 \%$, we aim for a total inclusion of 50 patients.

\section{Statistical analysis}

A non-linear mixed effects model will be used for analysis for cisplatin pharmacokinetics. Skeletal muscle mass will be analyzed as a continuous variable. The relationship between cisplatin pharmacokinetics, SMM, and BSA will be analyzed by introduction of these covariates into the pharmacokinetic model using standard model building techniques. Allometric relationships will be employed. Statistical significance will be assessed with the likelihood ratio test. Both statistical and graphical goodness-of-fit evaluations will be performed including visual predictive checks. An alternative cisplatin dosing scheme will be formulated if the results of the analysis allow for this. Association between cisplatin pharmacokinetics and treatment related toxicity and dose-limiting toxicity will be analyzed using multivariate logistic regression analysis. All statistical analyses will be performed using IBM SPSS Statistics version 21.0 software package (Chicago, Illinois, USA) or equivalent. Results will be considered statistically significant if the $p$-value was less than 0.05 . Continuous data will be represented as mean \pm standard deviation (SD) if normally distributed, and median \pm interquartile range (IQR) if skewed. Categorical data will be represented as a number and percentage of the total. Missing data will be simulated using multiple imputation to prevent possible bias due to missing data; however, we do not expect missing data to occur due to the nature and scale of the study. 


\section{Discussion}

Toxicity of cytotoxic agents is a common problem in medical oncology. In patients with advanced stage HNSCC treated with CRT with cisplatin, cisplatin related toxicity and dose limiting toxicity can results in decreased survival rates, as well as decreased quality of life and increased healthcare related costs. With many cytotoxic agents, it is difficult to predict which patients will experience severe toxicity. Over the last decade, many studies have been performed investigating the relationship between body composition and adverse outcomes in cancer patients. Specifically low SMM appears to be related with negative outcomes. Retrospective studies in patients with colorectal cancer, breast cancer, lung cancer and head and neck cancer, amongst others, have shown a relationship between low SMM before start of treatment and increased incidence of chemotherapy related toxicity and dose limiting toxicity. ${ }^{23,27,45}$

An explanation for this relationship that has been hypothesized, is that some cytotoxic drugs that are dosed based on the BSA of a patient only distribute into the fat-free body mass. Patients with low SMM and normal or high total body mass may actually receive a relatively high dose of these agents due to the limited distribution. This may explain the high incidence of chemotherapy related toxicity and doselimiting toxicity in patients with low SMM. On the other side, patients with high SMM and normal to low fat mass may also actually receive a relatively low cisplatin dose when the dose is calculated using BSA. Potentially, dose individualization based on the individual body composition of a patient may result in less severe toxicity and increased accuracy of the chemotherapy drug dose. However, the pharmacokinetic data that can prove this hypothesis are currently not available.

Our study will investigate this theory in a group of HNSCC patients treated with CRT with high dose cisplatin, a group of patients which often suffers from severe cisplatin related toxicity frequently resulting in treatment de-escalation. This study should provide results for the relationship between cisplatin pharmacokinetics, SMM and BSA in this patient group. All patients will receive standard-of-care CRT (three threeweekly cycles of cisplatin at $100 \mathrm{mg} / \mathrm{m}^{2} \mathrm{BSA}$ ) according to the standardized national treatment guidelines. Since this is an observational study, there will be no immediate reduction of toxicity and no direct benefit in terms of survival or quality of life in this study. Future gains may be significant in the treatment of advanced HNSCC patients in terms of survival benefit and quality of life. The results of this study may also be used in research in other types of cancer and chemotherapeutical drugs as a proofof-principle of the relationship between body composition and systemic exposure to chemotherapeutical drugs.

\section{References}

1. Bray F, Ferlay J, Soerjomataram I, Siegel RL, Torre LA, Jemal A. Global cancer statistics 2018: GLOBOCAN estimates of incidence and mortality worldwide for 36 cancers in 185 countries. CA Cancer J Clin. 2018;68(6):394-424.

2. Ferlay J, Soerjomataram I, Dikshit R, Eser S, Mathers C, Rebelo M, et al. Cancer incidence and mortality worldwide: sources, methods and major patterns in GLOBOCAN 2012. Int J Cancer. 2015;136(5):E359-86.

3. Nederlandse Kankerregistratie (NKR), IKNL [Dutch Cancer Registration, Netherlands Comprehensive Cancer Organisation] 2015 [10-01-2019]. Available from: https://www. cijfersoverkanker.nl/.

4. Argiris A, Eng C. Epidemiology, staging, and screening of head and neck cancer. Cancer Treat Res. 2003;114:15-60.

5. Argiris A, Karamouzis MV, Raben D, Ferris RL. Head and neck cancer. Lancet. 2008;371(9625):1695709.

6. Hashibe M, Brennan P, Chuang SC, Boccia S, Castellsague X, Chen C, et al. Interaction between tobacco and alcohol use and the risk of head and neck cancer: pooled analysis in the International Head and Neck Cancer Epidemiology Consortium. Cancer Epidemiol Biomarkers Prev. 2009;18(2):541-50.

7. Pezzuto F, Buonaguro L, Caponigro F, Ionna F, Starita N, Annunziata C, et al. Update on Head and Neck Cancer: Current Knowledge on Epidemiology, Risk Factors, Molecular Features and Novel Therapies. Oncology. 2015;89(3):125-36.

8. van Monsjou HS, Schaapveld M, van den Brekel MW, Balm AJ. The epidemiology of head and neck squamous cell carcinoma in The Netherlands during the era of HPV-related oropharyngea squamous cell carcinoma. Is there really evidence for a change? Oral Oncol 2015:51(10):901-7. Pignon JP, le Matre A, Maillard E Bourhis J, Group M-NC. Meta-analysis of chemotherapy in Cod 3 . Radiother Oncol. 2009:92(1):4-14.

10. Gregoire V, Lefebvre JL, Licitra L, Felip E, Group E-E-EGW. Squamous cell carcinoma of the head and neck: EHNS-ESMO-ESTRO Clinical Practice Guidelines for diagnosis, treatment and followup. Ann Oncol. 2010;21 Suppl 5:v184-6.

11. Blanchard P, Baujat B, Holostenco V, Bourredjem A, Baey C, Bourhis J, et al. Meta-analysis of chemotherapy in head and neck cancer (MACH-NC): a comprehensive analysis by tumour site. Radiother Oncol. 2011;100(1):33-40

12. Timme DW, Jonnalagadda S, Patel R, Rao K, Robbins KT. Treatment Selection for T3/T4a Laryngeal Cancer: Chemoradiation Versus Primary Surgery. Ann Otol Rhinol Laryngol. 2015;124(11):845-51.

13. Beijer YJ, Koopman M, Terhaard CH, Braunius WW, van Es RJ, de Graeff A. Outcome and toxicity of radiotherapy combined with chemotherapy or cetuximab for head and neck cancer: our experience in one hundred and twenty-five patients. Clin Otolaryngol. 2013;38(1):69-74.

14. de Jongh FE, van Veen RN, Veltman SJ, de Wit R, van der Burg ME, van den Bent MJ, et al. Weekly high-dose cisplatin is a feasible treatment option: analysis on prognostic factors for toxicity in 400 patients. Br J Cancer. 2003;88(8):1199-206.

15. Al-Mamgani A, de Ridder M, Navran A, Klop WM, de Boer JP, Tesselaar ME. The impact of cumulative dose of cisplatin on outcome of patients with head and neck squamous cell carcinoma. Eur Arch Otorhinolaryngol. 2017;274(10):3757-65.

16. Mehanna H, Robinson M, Hartley A, Kong A, Foran B, Fulton-Lieuw T, et al. Radiotherapy plus cisplatin or cetuximab in low-risk human papillomavirus-positive oropharyngeal cancer (DeESCALaTE HPV): an open-label randomised controlled phase 3 trial. Lancet. 2019;393(10166):51-60.

17. Taberna $\mathrm{M}$, Rullan AJ, Hierro C, Navarro V, Vazquez $\mathrm{S}$, Lozano A, et al. Late toxicity after radical treatment for locally advanced head and neck cancer. Oral Oncol. 2015;51(8):795-9.

18. Prado CM, Cushen SJ, Orsso CE, Ryan AM. Sarcopenia and cachexia in the era of obesity: clinical and nutritional impact. Proc Nutr Soc. 2016;75(2):188-98. 
19. Shen W, Punyanitya M, Wang Z, Gallagher D, St-Onge MP, Albu J, et al. Total body skeletal muscle and adipose tissue volumes: estimation from a single abdominal cross-sectional image. J Appl Physiol (1985). 2004;97(6):2333-8.

20. Prado CM, Lieffers JR, McCargar LJ, Reiman T, Sawyer MB, Martin L, et al. Prevalence and clinical implications of sarcopenic obesity in patients with solid tumours of the respiratory and gastrointestinal tracts: a population-based study. Lancet Oncol. 2008;9(7):629-35.

21. Levolger S, van Vugt JL, de Bruin RW, JN IJ. Systematic review of sarcopenia in patients operated on for gastrointestinal and hepatopancreatobiliary malignancies. Br J Surg. 2015;102(12):144858.

22. Pamoukdjian F, Bouillet T, Levy V, Soussan M, Zelek L, Paillaud E. Prevalence and predictive value of pre-therapeutic sarcopenia in cancer patients: A systematic review. Clin Nutr.

23. Rier HN, Jager A, Sleijfer S, Maier AB, Levin MD. The Prevalence and Prognostic Value of Low Muscle Mass in Cancer Patients: A Review of the Literature. Oncologist. 2016;21(11):1396-409.

24. Cruz-Jentoft AJ, Baeyens JP, Bauer JM, Boirie Y, Cederholm T, Landi F, et al. Sarcopenia: European consensus on definition and diagnosis: Report of the European Working Group on Sarcopenia in Older People. Age Ageing. 2010;39(4):412-23.

25. Hebuterne X, Lemarie E, Michallet M, de Montreuil CB, Schneider SM, Goldwasser F. Prevalence of malnutrition and current use of nutrition support in patients with cancer. JPEN J Parenter Enteral Nutr. 2014;38(2):196-204.

26. Wendrich AW, Swartz JE, Bril SI, Wegner I, de Graeff A, Smid EJ, et al. Low skeletal muscle mass is a predictive factor for chemotherapy dose-limiting toxicity in patients with locally advanced head and neck cancer. Oral Oncol. 2017;71:26-33.

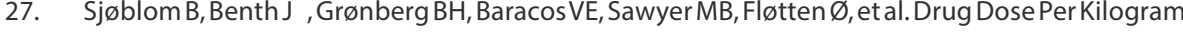
Lean Body Mass Predicts Hematologic Toxicity From Carboplatin-Doublet Chemotherapy in Advanced Non-Small-Cell Lung Cancer. Clin Lung Cancer. 2017 Mar;18(2):129-136.

28. Hopkins J, Sawyer MB. A review of body composition and pharmacokinetics in oncology. Expert Rev Clin Pharmacol. 2017;10(9):947-56.

29. Carneiro IP, Mazurak VC, Prado CM. Clinical Implications of Sarcopenic Obesity in Cancer. Curr Oncol Rep. 2016;18(10):62.

30. Prado CM, Heymsfield SB. Lean tissue imaging: a new era for nutritional assessment and intervention. JPEN J Parenter Enteral Nutr. 2014;38(8):940-53.

31. Felici A, Verweij J, Sparreboom A. Dosing strategies for anticancer drugs: the good, the bad and body-surface area. Eur J Cancer. 2002;38(13):1677-84.

32. Mathijssen RH, de Jong FA, Loos WJ, van der Bol JM, Verweij J, Sparreboom A. Flat-fixed dosing versus body surface area based dosing of anticancer drugs in adults: does it make a difference? Oncologist. 2007;12(8):913-23.

33. de Jongh FE, Verweij J, Loos WJ, de Wit R, de Jonge MJ, Planting AS, et al. Body-surface areabased dosing does not increase accuracy of predicting cisplatin exposure. J Clin Oncol. 2001;19(17):3733-9. DOI: 10.1200/JCO.2001.19.17.3733

34. Farris FF, Dedrick RL, King FG. Cisplatin pharmacokinetics: applications of a physiological model. Toxicol Lett. 1988 Oct;43(1-3):117-37.

35. Go RS, Adjei AA. Review of the comparative pharmacology and clinical activity of cisplatin and carboplatin. J Clin Oncol. 1999;17(1):409-22.

36. Swartz JE, Pothen AJ, Wegner I, Smid EJ, Swart KM, de Bree R, et al. Feasibility of using head and neck CT imaging to assess skeletal muscle mass in head and neck cancer patients. Oral Oncol. 2016;62:28-33.

37. Heymsfield SB, Wang Z, Baumgartner RN, Ross R. Human body composition: advances in models and methods. Annu Rev Nutr. 1997; 17:527-58.

38. Brouwers $E$ E Tibben MM, Rosing $H_{1}$ Hillebrand $M J$, Joerger $M$, Schellens $J H$ et al. Sensitive inductively coupled plasma mass spectrometry assay for the determination of platinum originating from cisplatin, carboplatin, and oxaliplatin in human plasma ultrafiltrate. I Mass
39. Bolke E, Schieren G, Gripp S, Steinbach G, Peiper M, Orth K, et al. Cystatin C - a fast and reliable Onkologie : Organ der Deutschen Rontgengesellschaft [et al]. 2011;187(3):191-201.

40. Inker LA, Schmid $\mathrm{CH}$, Tighiouart $\mathrm{H}$, Eckfeldt JH, Feldman $\mathrm{HI}$, Greene $\mathrm{T}$, et al. Estimating glomerular filtration rate from serum creatinine and cystatin C. N Engl J Med. 2012;367(1):20-9.

41. Feliciano EMC, Kroenke CH, Meyerhardt JA, Prado CM, Bradshaw PT, Kwan ML, et al. Association of Systemic Inflammation and Sarcopenia With Survival in Nonmetastatic Colorectal Cancer: Results From the C SCANS Study. JAMA Oncol. 2017;3(12):e172319.

42. Curcio F, Ferro G Basile $C$, Liguori I, Parrella P, Pirozzi $F$, et al. Biomarkers in sarcopenia: $A$ multifactorial approach. Exp Gerontol. 2016;85:1-8.

43. van Vugt JLA, Buettner $S$, Levolger S, Coebergh van den Braak RRJ, Suker M, Gaspersz MP, et al. Low skelat muscle mass is associated with increased hospital expenditure in patients undergoing cancer surgery of the alimentary tract. PLoS One. 2017;12(10):e0186547.

40. Go RS, Adjei AA Review of the comparative pharmacology and clinical activity of cisplatin and carboplatin. J Clin Oncol. 1999;17(1):409-22.

44. Urien S, Lokiec F. Population pharmacokinetics of total and unbound plasma cisplatin in adult patients. Br J Clin Pharmacol. 2004;57(6):756-63.

45. Hilmi M, Jouinot A, Burns R, Pigneur F, Mounier R, Gondin J, et al Body composition and 作, Pharmacol Ther. 2018:S0163-7258(18) 30219-5. 


\section{Chapter 11}

Summarizing discussion 
The aims of this thesis were to investigate the use of diagnostically performed imaging of the head and neck area to assess total body composition, and to evaluate the clinical relevance of these features in head and neck cancer patients on treatment outcome and survival. We predominantly focused on radiologically assessed skeletal muscle mass prior to treatment, which is proposed as a biomarker for biological and functional fitness. We also included the radiological assessment of arterial calcification formation as an indicator of vascular status. The results of this thesis may allow for personalized medicine in terms of upfront treatment adaptation and optimization of the risk assessment on adverse outcomes of treatment, in a patient population known to be at high risk of adverse clinical outcomes.

In oncological research, the body composition and specifically skeletal muscle mass of cancer patients is commonly assessed on abdominal computed tomography (CT) imaging at the level of the third lumbar vertebra (L3). ${ }^{1,2}$ Abdominal imaging has proven to be both accurate in terms of relationship with whole body skeletal muscle mass, as well as easily applicable in daily practice; in most cancer patients, abdominal CT imaging is routinely performed for diagnostic purposes. ${ }^{3,4}$ In head and neck cancer, abdominal CT imaging is often not performed; thus, this assessment method for body composition is not applicable in head and neck cancer patients without additional imaging of the abdomen. In a study by Swartz et al, measurement of skeletal muscle mass at the level of the third cervical vertebra (C3) was postulated, using routinely performed imaging of the head and neck area.

In Part I of this thesis, a general introduction on the history and relevance of body composition research in oncology is proffered, and the rationale for the other parts of this thesis is outlined.

Part II of this thesis was aimed to further investigate the validity of the measurement method of skeletal muscle mass at the level of $\mathrm{C} 3$ on routinely performed CT imaging or magnetic resonance imaging (MRI) of the head and neck area, in terms of accuracy, robustness and interobserver variability.

In Chapter 2, the correlation between skeletal muscle mass at the level of C3 and L3 was reexamined, to validate the results found in the study by Swartz et al. The results of this study show that the correlation between cross-sectional muscle area at the level of $\mathrm{C} 3$ and $\mathrm{L} 3$ was good $(r=0.75)$. Using the multivariable prediction formula for cross-sectional muscle area at the level of $L 3$ from cross-sectional muscle area at $\mathrm{C}_{3}$, the agreement was excellent $(r=0.82)$. There was some difference in the identification of patients with low skeletal muscle mass based on the estimated lumbar skeletal muscle index and the actual lumbar skeletal muscle index (Cohen's k: 0.57 ), although the sensitivity of a score of low skeletal muscle mass using the estimated lumbar skeletal muscle index was high (84.4\%).

In Chapter 3, the interobserver agreement for skeletal muscle mass measurement at the level of $\mathrm{C} 3$ was investigated. The results of this study show that there is excellent intraclass correlation between observers for skeletal muscle area at the level of C3 (intraclass correlation coefficients $0.76-0.97$; all $p<0.01$ ). There was some difference in specific $C T$ slice selection (Fleiss' $\mathrm{k}: 0.61$ ), but the agreement in vertebra selection was excellent (Fleiss' K: 0.96); the differences in single slice selection were deemed clinically insignificant. The interobserver agreement for skeletal muscle mass measurement at the level of $\mathrm{C} 3$ was excellent.

In Chapter 4, the relationship between whole body skeletal muscle volume as measured on whole body MRI and cross-sectional skeletal muscle area at the level of C3 was investigated using a previously established database of healthy volunteers who underwent whole body MRI for research purposes. ${ }^{4}$ The usage of the cross-sectional muscle area at the level of L3 is based on whole body MRI databases, where a single transversal slice at the level of $\mathrm{L} 3$ has proven to provide the best correlation with whole body skeletal muscle volumes. Although the cross-sectional muscle area at the level of $\mathrm{C} 3$ has shown to correlate well with cross-sectional muscle area at the level of $\mathrm{L} 3$, its relationship with whole body skeletal muscle volume as measured with whole body MRI was not yet established. The results of this study provide evidence that there is an excellent correlation between cross-sectional muscle area at the level of $\mathrm{C} 3$ and whole body skeletal muscle volume $(r=0.89)$. We formulated a multivariable prediction formula for whole body skeletal muscle volume based on cross-sectional muscle area at the level of C3 with a high accuracy ( $r=0.94$, standard error 2.2 liters).

From these studies in Part II of this thesis we conclude that a measurement of skeletal muscle mass at the level of C3 is robust and easy to use, using routinely performed CT imaging of the head and neck area. Skeletal muscle mass at the leve of $\mathrm{C} 3$ can be used as an estimation of whole body skeletal muscle mass . It allows for retrospective and prospective research into the predictive and prognostic value of low skeletal muscle mass in the vast majority of head and neck cancer patients, as well as use in possible future trials. 
In Part III of this thesis, the relationship between radiologically assessed body composition and short- and long-term clinical outcomes was explored in patient with head and neck squamous cell carcinoma at different localizations and undergoing different types of treatment. The treatment of locally advanced head and neck cancer is often with curative intent, but at the expensive of significant treatment related toxicity and morbidity. ${ }^{6}$ In patients undergoing primary chemoradiotherapy, approximately $30 \%$ of patients experience chemotherapy dose-limiting toxicity. ${ }^{78}$ In patients undergoing total laryngectomy, up to 30\% of patients experience a pharyngocutaneous fistula after treatment, associated with significant morbidity and mortality, as well as increased hospital stay and decreased quality of life. ${ }^{9-11}$ Despite current risk assessment strategies, novel risk factors for the accurate identification of patients at high risk of adverse outcomes are wanted.

In several other studies in cancer patients, including studies in patients with breast cancer, colorectal cancer and lung cancer, a relationship between low skeletal muscle mass and chemotherapy related toxicity and dose-limiting toxicity is systematically found. ${ }^{12-14}$ In head and neck cancer patients undergoing chemoradiotherapy with platinum-based chemotherapy, a study by Wendrich et al showed that patients with low skeletal muscle mass as measured at the level of C3 had a trifold incidence of chemotherapy dose-limiting toxicity compared to patients with normal skeletal muscle mass. ${ }^{15}$ In Chapter 5, the relationship between low skeletal muscle mass and chemotherapy dose-limiting toxicity was investigated in a different cohort of patients with locally advanced head and neck cancer undergoing primary chemoradiotherapy with cisplatin. In this study, 54.9\% of patients had low skeletal muscle mass. The results showed that patients with low skeletal muscle mass significantly more often experienced chemotherapy dose-limiting toxicity; in fact, the incidence of chemotherapy dose-limiting toxicity again was trifold in patients with low skeletal muscle mass compared to patients with normal skeletal muscle mass ( $35.7 \%$ versus $10.1 \%, p<0.01$ for patients with low and normal skeletal muscle mass, respectively); the odds ratio for occurrence of chemotherapy dose limiting toxicity in patients with low skeletal muscle mass was 3.99 (95\% confidence interval $1.56-10.23, p=0.01)$ in multivariable analysis. In terms of survival, we did not find a relationship between low skeletal muscle mass and decreased overall survival. However, we did find that chemotherapy dose-limiting toxicity was significantly associated with a decreased overall survival in patients with HPV-negative head and neck cancer (hazard ratio $2.10,95 \%$ confidence interval 1.13 - 3.90) in multivariable analysis.
In Chapter 6, the relationship between low skeletal muscle mass and postoperative complications including pharyngocutaneous fistula formation as well as overall survival, in patients undergoing total laryngectomy for any reason was investigated. In surgical oncology, a plethora of evidence has been published relating a low skeletal muscle mass with an increased incidence of postoperative complications. ${ }^{13,16,17}$ In our current study, $46.4 \%$ of patients undergoing laryngectomy had low skeletal muscle mass. The difference in overall postoperative complications was not significant between patients with low and normal skeletal muscle mass (all grade complications: $67.9 \%$ versus $61.1 \%$ for patients with low skeletal muscle mass and normal skeletal muscle mass, respectively). Patients with low skeletal muscle mass did significantly more often experience pharyngocutaneous fistula formation $(34.9 \%$ vs $20.6 \%$; $p=0.02)$; low skeletal muscle mass was a significant predictor for pharyngocutaneous fistula formation (odds ratio 1.95, 95\% confidence interval 1.04 - 3.66) in multivariable analysis. These results are in concurrence with a study by Achim et al, who also showed that low skeletal muscle mass as measured at the level of $L 3$ was predictive of wound complications including pharyngocutaneous fistula. ${ }^{18}$ Low skeleta muscle mass was significantly associated with a decreased overall survival (hazard ratio $1.85,95 \%$ confidence interval $1.20-2.84$ ). A study in patients undergoing surgery for abdominal cancer showed that hospital stay was significantly longer and healthcare expenditures significantly higher in patients with low skeletal muscle mass. ${ }^{19}$ We found that patients with low skeletal muscle mass had significantly longer duration of hospital stay than patients with normal skeletal muscle mass (median 17 days versus 14 days for patients with low and norma skeletal muscle mass, respectively; $\mathrm{p}<0.01$ ); it can be anticipated that healthcare related expenditures were also higher in patients with low skeletal muscle mass.

In geriatrics, sarcopenia is known as an age-related syndrome with a multifactorial etiology, characterized by generalized loss of skeletal muscle mass and loss of muscle strength or function. According to the EWGSOP, sarcopenia was therefore defined as a coexistence of low skeletal muscle mass and low muscle function. In geriatric research, sarcopenia is associated with decreased quality of life and early death. ${ }^{20} \mathrm{~A}$ study in gastric cancer patients undergoing surgery showed that sarcopenia, as defined by both low skeletal muscle mass and low muscle function was associated with a higher complication rate after surgery. ${ }^{21}$ Chapter 7 nvestigated the relationship between sarcopenia and survival in elderly patients with head and neck cancer. In this study, $81.2 \%$ of patients had low skeletal muscle mass; $48.2 \%$ of patients were defined as having sarcopenia (defined as the combination of low skeletal muscle mass and low muscle function). The 
results showed that overall survival for patients treated with curative intent was significantly worse in those who had sarcopenia, versus in those who did not have sarcopenia (median survival 12.1 months versus 13.6 months; hazard ratio 2.80 , $95 \%$ confidence interval $1.14-6.88, p=0.03$ ). Low skeletal muscle mass only and low muscle function only were not associated with overall survival separate from each other.

Low skeletal muscle mass has widely been described as a negative prognostic factor in oncology. In Chapter 8, the prognostic value of low skeletal muscle mass in patients with oropharyngeal cancer treated with curative intent is investigated Also investigated was the relevance of a state of 'sarcopenic obesity', in which both low skeletal muscle mass and obesity are present. ${ }^{2}$ In literature, the presence of sarcopenic obesity has been associated with a markedly increased risk of severe complications and decreased survival. In our study, low skeletal muscle mass was present in $64.8 \%$ of patients and sarcopenic obesity was present in $6 \%$ of patients. Low skeletal muscle mass and sarcopenic obesity were both associated with a decreased overall survival. Particularly in sarcopenic obesity, this effect was pronounced, even when corrected for known other prognosticators including high risk HPV-status.

Recent studies in patients with esophageal cancer have shown that arteria calcification as assessed on routinely performed CT imaging was associated with postoperative wound complications. ${ }^{22,23}$ Research has shown that the presence of vascular calcium on CT imaging performed for another purpose may be used as a biomarker of cardiovascular disease..$^{24}$ In Chapter 9, the incidence of CT assessed arterial calcifications at ten different anatomical locations in patients undergoing laryngectomy is investigated, and its predictive significance for pharyngocutaneous fistula formation is evaluated. The results of this study showed that arterial calcifications are a common finding in patients undergoing laryngectomy, with only $1.3 \%$ percent of patients having no arterial calcification present and $7.1 \%$ of patients having at most mild arterial calcifications present. Arterial calcifications at several locations, most notably of the descending aorta and origo of the brachiocephalic arteries, were significantly associated with pharyngocutaneous fistula formation. A higher total arterial calcification score was also significantly associated with pharyngocutaneous fistula formation.

In Part IV of this thesis, a study is proposed to investigate the mechanisms as to why low skeletal muscle mass is associated with the occurrence of chemotherapy dose-limiting toxicity in patients undergoing primary chemoradiotherapy with cisplatin. A hypothesis for this phenomenon is that the pharmacokinetics of cisplatin is altered with respect to the distributional volume in patients with a low skeletal muscle mass and normal to high adipose tissue mass. Cisplatin is a hydrophilic chemotherapeutical agent, and mainly distributes into the fat-free body mass, of which skeletal muscle mass is the largest component. ${ }^{25-27}$ Cisplatin is dosed using the body surface area of a patient, and does not take into account individual body composition. ${ }^{28,29}$ It is therefore hypothesized that patients with low skeletal muscle mass and normal or high adipose tissue mass may actually receive a relatively high dose of cisplatin. Data on the relationship between body composition and pharmacokinetic characteristics of cisplatin is not yet available.

In Chapter 10, we propose at study to investigate the association between cisplatin pharmacokinetics, skeletal muscle mass and body surface area in head and neck cancer patients treated with chemoradiotherapy with cisplatin. The results of this study will provide clarity into the relationship between low skeleta muscle mass, cisplatin pharmacokinetics and cisplatin toxicity. The findings may be used to individualize cisplatin treatment in head and neck cancer patients based on the body composition of an individual patient. The results of this study may also be used as a proof-of-principle in research in other types of cancer and chemotherapeutical agents. 


\section{General discussion}

In recent years, it has been shown that routinely performed imaging, such as computed tomography (CT) scans, can be used to extract additional information on patient's functional and biological status, which may be used to identify patients at increased risk of adverse outcomes during and after treatment. Low skeletal muscle mass is the most known example of this application; research has shown that modern imaging such as CT or MRI can be used to accurately assess skeletal muscle quantity. Our research shows that the quantity of skeletal muscle mass can easily and reliably be assessed on CT imaging of the head and neck area. Two recent studies showed that head and neck MRI can also be used to accurately measure skeletal muscle mass at the level of $\mathrm{C} 3 .^{30,31}$ Low skeletal muscle mass, as identified on routinely performed CT and MRI of the head and neck area, is associated with increased chemotherapy dose-limiting toxicity, increased pharyngocutaneous fistula formation after total laryngectomy and decreased overall survival in several head and neck patient categories. These results are in concurrence with results in other types of cancer. Routinely performed imaging can also be used to assess the presence of arterial calcification, as a proxy for cardiovascular disease.

Concluding, routinely performed imaging does not only provide easily accessible and clinically relevant information on disease status, but also holds valuable information on body composition of a patient, which may be used in individualized risk stratification, treatment adaptation and patient optimization strategies to ultimately decrease short-term adverse outcomes and increase survival.

\section{Future perspectives}

Future studies should be aimed at three main research themes. First, it should be explored if and to what extent it is possible to adapt head and neck cancer treatment to a patient's individual body composition, with the aim that negative outcomes associated with body composition are decreased and the given treatment is still at least as effective as current standard-of-care. In patients undergoing chemoradiotherapy with cisplatin, the results of the study proposed in Chapter 10 should provide the information whether a chemotherapy dose modification based on skeletal muscle mass may result in less chemotherapy related toxicity and dose-limiting toxicity, and maximize cumulative cisplatin dose administered.
An alternative may be that low skeletal muscle mass is used as an argument for an intended treatment de-escalation choice, such as weekly low-dose cisplatin to maximize treatment adherence and cumulative cisplatin dose administered by decreasing the risk of dose-limiting toxicity occurring. In surgically treated patients, such as patients undergoing laryngectomy, preemptive supportive tissue flaps such as an overlay pectoralis major flap may be used in patients with low skeletal muscle mass, to decrease the risk of fistulation. ${ }^{32}$ Randomized controlled trials should be performed to provide the evidence that treatment adaptation leads to decreased adverse outcomes with equal or better efficacy.

Secondly, it should be explored whether interventions are able to improve body composition in head and neck cancer patients with low skeletal muscle mass. The effect of these interventions should be investigated before and during treatment There is a need for clinical research to clarify whether low skeletal muscle mass is prognostic only, or that improving a patient's body composition will decrease the risk of adverse outcomes. Treatment may be offered to increase and adapt skeletal muscle mass and overall body composition before or during treatment and to increase overall physical fitness. This approach should be multimodal, focusing on physical therapy as well as nutritional support. A complicating factor is that the pre-treatment time window is typically short, with about 4 weeks tota time from first referral to the outpatient clinic to start of treatment. In surgically treated patients, the pre-treatment optimization of physical and nutritiona condition, sometimes termed prehabilitation, has become a point of interest. In patients with hepatobiliary, colorectal and upper gastrointestinal cancer treated with surgery, a recent meta-analysis found that duration of hospital stay was significantly shorter in patients participating in a prehabilitation program which generally consisted of a prehabilitation time period of less than 4 weeks. ${ }^{33}$ While no significant difference in complication rate or survival rate was found, other studies have noted a trend towards less complications. ${ }^{34}$ In other types of cancer, including rectal cancer and breast cancer, randomized controlled trials showed that exercise interventions during chemotherapy show a positive effect on treatment tolerance and fatigue, and may increase skeletal muscle mass..$^{35-37}$ Feasibility studies in patients with head and neck cancer patients have shown that muscle resistance training programs in patients undergoing chemoradiotherapy or radiotherapy are feasible and show high patient satisfaction. ${ }^{38,39}$ Whether such interventions provide benefit in terms of overall survival is unknown, but low skeletal muscle mass prior to start of treatment may be an indicator that a patient may benefit from intensified supportive care in terms of physical exercise and nutritional support. Implementation of additional supportive care in daily clinical 
practice may contribute to patient fitness and overall well-being, may decrease length of hospital stay, complications of surgery and toxicities of treatment and might ultimately also contribute to increased survival.

MRI and CT imaging is regularly performed during diagnosis and follow up of cancer patients to assess tumor status and anatomic boundaries but research shows that it also harbors a considerable amount of information on a patient's biological and functional status; such as, but not limited to, skeletal muscle mass and quality. This information is currently not used to its full extent and the patient does not benefit from this knowledge that could be available. So thirdly, to allow the clinician to routinely use this information to optimize a patient's treatment or risk assessment, software programs should become available to process the additional information present on our routinely performed imaging The development of automatic body composition analysis software based on CT or MRI should be pursued. Research into this subject using deep-learning neura networks is currently ongoing for automatic skeletal muscle mass assessment on abdominal CT imaging, and for calcium scoring on thoracic CT imaging. ${ }^{40-42}$ For head and neck cancer patients, research should focus on how to optimally use head and neck imaging to extract additional information on a patient's body composition from the imaging that is available.

\section{References}

. Prado CM, Lieffers JR, McCargar LJ, et al. Prevalence and clinical implications of sarcopenic obesity in patients with solid tumours of the respiratory and gastrointestinal tracts: population-based study. Lancet Oncol. 2008;9(7):629-635.

2. Prado CM, Cushen SJ, Orsso CE, Ryan AM. Sarcopenia and cachexia in the era of obesity: clinica and nutritional impact. Proc Nutr Soc. 2016;75(2):188-198.

3. Shen W, Punyanitya M, Wang Z, et al. Total body skeletal muscle and adipose tissue volumes: estimation from a single abdominal cross-sectional image. J Appl Physiol. 2004;97(6):23332338. to assess whole-body skeletal muscle and adipose tissue volumes in healthy adults?1. Am Clin Nutr. 2015;102(1):58-65.

5. SWartz JE, Pothen AJ, Wegner l, et al. Feasibility of using head and neck CT imaging to assess skeletal muscle mass in head and neck cancer patients. Oral Oncol. 2016;62:28-33.

6. Grégoire V, Lefebvre J-L, Licitra L, Felip E. Squamous cell carcinoma of the head and neck: EHNSXESMOXESTRO Clinical Practice Guidelines for diagnosis, treatment and follow-up. Ann Oncol. 2010;21:v184-v186.

7. Szturz P, Wouters K, Kiyota N, et al. Low-Dose vs. High-Dose Cisplatin: Lessons Learned From 59 Chemoradiotherapy Trials in Head and Neck Cancer. Front Oncol. 2019;9.

8. Forastiere AA, Zhang Q, Weber RS, et al. Long-Term Results of RTOG 91-11: A Comparison of Three Nonsurgical Treatment Strategies to Preserve the Larynx in Patients With Locally Advanced Larynx Cancer. J Clin Oncol. 2013;31(7):845-852.

9. Lansaat $\mathrm{L}$, van der Noort $\mathrm{V}$, Bernard $\mathrm{SE}_{\mathrm{B}}$ et al. Predictive factors for pharyngocutaneous fistulization after total laryngectomy: a Dutch Head and Neck Society audit. Eur Arch OtoRhino-Laryngology. 2018;275(3):783-794.

10. Dedivitis RA, Aires FT, Cernea CR, Brandão LG. Pharyngocutaneous fistula after total laryngectomy: Systematic review of risk factors. Head Neck. 2015 Nov;37(11):1691-7.

11. Hasan Z, Dwivedi RC, Gunaratne DA, Virk SA, Palme CE, Riffat F. Systematic review and metaanalysis of the complications of salvage total laryngectomy. Eur J Surg Oncol. 2017;43(1):42-51.

Rier HN, Jager A, Sleijfer S, Maier AB, Levin M. The Prevalence and Prognostic Value of Low Muscle Mass in Cancer Patients: A Review of the Literature Oncologist. 2016-21(11):1396-1409.

3. Levolger $S$, van Vugt JLA, de Bruin RWF, IJzermans JNM. Systematic review of sarcopenia in patients operated on for gastrointestinal and hepatopancreatobiliary malignancies. Br I Surg. 2015;102(12):1448-1458.

14. Pamoukdjian F, Bouillet T, Lévy V, Soussan M, Zelek L, Paillaud E. Prevalence and predictive value of pre-therapeutic sarco sonia in cancer patients: A systematic review. Clin Nutr. 101-1113.

15. Wendrich AW, Swartz JE, Bril SI, et al. Low skeletal muscle mass is a predictive factor for chemotherapy dose-limiting toxicity in patients with locally advanced head and neck cancer. Oral Oncol. 2017;71:26-33.

16. Weerink LBM, van der Hoorn A, van Leeuwen BL, de Bock GH. Low skeletal muscle mass and postoperative morbidity in surgical oncology: a systematic review and meta-analysis. Cachexia Sarcopenia Muscle. 2020 Jun;11(3):636-649.

17. Jones K, Gordon-Weeks A, Coleman C, Silva M. Radiologically Determined Sarcopenia Predicts Morbidity and Mortality Following Abdominal Surgery: A Systematic Review and MetaAnalysis. World J Surg. 2017;41(9):2266-2279.

18. Achim V, Bash J, Mowery A, et al. Prognostic Indication of Sarcopenia for Wound Complication After Total Laryngectomy. JAMA Otolaryngol Neck Surg. 2017;143(12):1159.

19. van Vugt JLA, Buettner $S$, Levolger $S$, et al. Low skeletal muscle mass is associated with increased hospital expenditure in patients undergoing cancer surgery of the alimentary tract. Bianchi $C$ ed. PLoS One. 2017;12(10):e0186547. 
20. Cruz-Jentoft AJ, Baeyens JP, Bauer JM, et al. Sarcopenia: European consensus on definition and diagnosis: Report of the European Working Group on Sarcopenia in Older People. Age Ageing 2010;39(4):412-423.

21. Fukuda $Y$, Yamamoto K, Hirao M, et al. Sarcopenia is associated with severe postoperative complications in elderly gastric cancer patients undergoing gastrectomy. Gastric Cancer. Published online 2016

22. Borggreve AS, Goense L, van Rossum PSN, van Hillegersberg R, de Jong PA, Ruurda JP. Generalized cardiovascular disease on a preoperative $C T$ scan is predictive for anastomotic leakage after esophagectomy. Eur J Surg Oncol. 2018;44(5):587-593.

23. Goense L, van Rossum PSN, Weijs TJ, et al. Aortic Calcification Increases the Risk of Anastomotic Leakage After Ivor-Lewis Esophagectomy. Ann Thorac Surg. 2016;102(1):247-252.

24. Waltz J, Kocher M, Kahn J, Dirr M, Burt JR. The Future of Concurrent Automated Coronary Artery Calcium Scoring on Screening Low-Dose Computed Tomography. Cureus. 2020 Ju 12;12(6):e8574.

25. Prado CMM, Heymsfield SB. Lean Tissue Imaging. J Parenter Enter Nutr. 2014;38(8):940-953.

6. Farris FF, Dedrick RL, King FG. Cisplatin pharmacokinetics: applications of a physiologica model. Toxicol Lett. 1988 Oct; $43(1-3): 117-37$.

27. Go RS, Adjei AA. Review of the comparative pharmacology and clinical activity of cisplatin and carboplatin. J Clin Oncol. 1999 Jan;17(1):409-22

28. De Jongh FE, Verweij J, Loos WJ, et al. Body-surface area-based dosing does not increase accuracy of predicting cisplatin exposure. J Clin Oncol. 2001 Sep 1;19(17):3733-9.

9. Urien S, Lokiec F. Population pharmacokinetics of total and unbound plasma cisplatin in adult patients. Br J Clin Pharmacol. 2004;57(6):756-763.

30. Chargi N, Ansari E, Huiskamp LFJ, Bol G, de Bree R. Agreement between skeletal muscle mass measurements using computed tomography imaging and magnetic resonance imaging in head and neck cancer patients. Oral Oncol. 2019;99:104341.

31. Zwart AT, Becker J-N, Lamers MJ, et al. Skeletal muscle mass and sarcopenia can be determined with 1.5-T and 3-T neck MRI scans, in the event that no neck CT scan is performed. Eur Radiol. 2021;31(6):4053-4062.

32. Gil Z, Gupta A, Kummer B, et al. The Role of Pectoralis Major Muscle Flap in Salvage Tota Laryngectomy. Arch Otolaryngol Neck Surg. 2009;135(10):1019.

33. van Waart $\mathrm{H}$, Stuiver MM, van Harten WH, et al. Effect of Low-Intensity Physical Activity and Moderate- to High-Intensity Physical Exercise During Adjuvant Chemotherapy on Physica Fitness, Fatigue, and Chemotherapy Completion Rates: Results of the PACES Randomized Clinical Trial. J Clin Oncol. 2015;33(17):1918-1927.

34. Lambert JE, Hayes LD, Keegan TJ, et al. The Impact of Prehabilitation on Patient Outcomes in Hepatobiliary, Colorectal, and Upper Gastrointestinal Cancer Surgery: A PRISMA-Accordant Meta-analysis. Ann. Surg. 2021;274(1):70-77

35. Dewulf M, Verrips $M$, Coolsen MME, et al. The effect of prehabilitation on postoperative complications and postoperative hospital stay in hepatopancreatobiliary surgery a systematic review. HPB (Oxford). 2021;S1365-182X(21)00131-3.

36. Moug SJ, Barry SJE, Maguire $\mathrm{S}$, et al. Does prehabilitation modify muscle mass in patients with rectal cancer undergoing neoadjuvant therapy? A subanalysis from the REx randomised controlled trial. Tech Coloproctol. 2020;24(9):959-964.

37. Mijwel S, Bolam KA, Gerrevall J, Foukakis T, Wengström Y, Rundqvist H. Effects of Exercise on Chemotherapy Completion and Hospitalization Rates: The OptiTrain Breast Cancer Trial. Oncologist. 2020;25(1):23-32.

38. Sandmael JA, Bye A, Solheim TS, et al. Feasibility and preliminary effects of resistance training and nutritional supplements during versus after radiotherapy in patients with head and neck cancer: A pilot randomized trial. Cancer. 2017;123(22):4440-4448.

39. Brown TE, Banks MD, Hughes BGM, Lin CY, Kenny LM, Bauer JD. Randomised controlled trial of early prophylactic feeding vs standard care in patients with head and neck cancer. Br $S C$ 2017;117(1):15-24.
40. Weston AD, Korfiatis $P$, Kline $T L$, et al. Automated Abdominal Segmentation of CT Scans for Body Composition Analysis Using Deep Learning. Radiology. 2019;290(3):669-679.

41. Koitka S, Kroll L, Malamutmann E, Oezcelik A, Nensa F. Fully-automated body composition analysis in routine ct imaging using $3 \mathrm{~d}$ semantic segmentation convolutional neural networks. ur Radiol. 2021 Apr;31(4):1795-1804.

42. Gernaat SAM, Išgum I, de Vos BD, et al. Automatic Coronary Artery Calcium Scoring on Radiotherapy Planning CT Scans of Breast Cancer Patients: Reproducibility and Association with Traditional Cardiovascular Risk Factors. Zirlik A, ed. PLoS One. 2016;11(12):e0167925. 


\section{APPENDICES}




\section{Chapter 12}

Dutch summary -

Nederlandse samenvatting 


\section{Skeletspiermassa bij hoofd-halskankerpatiënten: radiologische beoordeling en relatie met klinische uitkomsten}

Het hoofd-halsplaveiselcelcarcinoom (HHPCC) is een verzameling van tumoren uitgaande van verschillende lokalisaties in het hoofd-halsgebied met voor elke lokalisatie specifieke symptomen, diagnostische onderzoeken en behandelingen. Wereldwijd is HHPCC de 8e meest voorkomende soort van kanker. ${ }^{1}$ De incidentie van HHPCC in Nederland is jaarlijks ongeveer 3000, en ruim 900 mensen overlijden jaarlijks hieraan. De behandeling van HHPCC is mede afhankelijk van de locatie van het HHPCC en het stadium waarin de ziekte zich presenteert, maar is vaak multimodaal en kan chirurgie, chemotherapie en/of radiotherapie bevatten. Gemetastaseerde ziekte bij diagnose komt weinig voor, dus de behandeling is vaak met curatieve intentie. De behandeling gaat helaas ook vaak gepaard met complicaties en toxiciteit. Bij patiënten die voor een larynxcarcinoom een totale laryngectomie ondergaan, is een faryngocutaan fistel een gevreesde en frequent voorkomende postoperatieve complicatie. ${ }^{2}$ De behandeling van locoregionaal gevorderd HHPCC met chemotherapie en radiotherapie kent veel bijwerkingen, waardoor patiënten soms voortijdig moeten stoppen met de behandeling. ${ }^{3}$

Het laatste decennium is er in toenemende mate aandacht voor de lichaamssamenstelling van patiënten, en de verhouding tussen skeletspiermassa en vetmassa. Een veelbelovende biomarker voor het voorspellen van nadelige uitkomsten zoals postoperatieve complicaties en chemotherapie gerelateerde toxiciteit, als ook een slechtere overleving, is een lage skeletspiermassa. ${ }^{4}$ In de oncologische literatuur wordt frequent gebruik gemaakt van een meting van skeletspieroppervlakte op een enkele CT coupe op het niveau van lumbale wervel $L 3$, als indicator van totale lichaamssamenstelling. ${ }^{5}$ In eerder onderzoek waarbij gebruikt werd gemaakt van MRI van het hele lichaam (whole body MRI), werd gevonden dat skeletspieroppervlakte op een enkele coupe ter hoogte van L3 de beste relatie had met totale lichaam skeletspiervolume zoals gemeten met whole body MRI. ${ }^{6}$ Bij HHPCC patiënten is er vaak geen CT beeldvorming van L3 aanwezig. Door Swartz et al werd een methode beschreven om skeletspiermassa te meten op CT beeldvorming van het hoofd-hals gebied, ter hoogte van de derde cervicale wervel C3. ${ }^{7}$ Deze wervel staat afgebeeld op routinematig vervaardigde beeldvorming bij HHPCC patiënten. Wendrich et al gebruikten deze methode om een afkapwaarde voor lage skeletspiermassa bij HHPCC patiënten te formuleren, en om de relatie tussen lage skeletspiermassa en chemotherapie dosis-limiterende toxiciteit te onderzoeken. ${ }^{8}$ In dit onderzoek bleek lage skeletspiermassa voorspellend voor het optreden van chemotherapie dosislimiterende toxiciteit.

In dit proefschrift wordt onderzoek beschreven naar het meten van skeletspiermassa bij HHPCC patiënten op CT beeldvorming op het niveau van wervel C3, en de associatie van lage skeletspiermassa gemeten op niveau C3 met negatieve behandeluitkomsten. De resultaten van dit proefschrift dragen bij aan een verbeterde risicostratificatie bij HHPCC patiënten voorafgaand aan behandeling en een verbeterde inschatting van de prognose. Daarnaast kunnen deze gebruikt worden om de behandeling te optimaliseren voor de individuele lichaamssamenstelling van een patiënt.

In Deel I van dit proefschrift wordt een algemene introductie gegeven over HHPCC en de behandeling hiervan, en een introductie over het onderwerp lichaamssamenstelling en skeletspiermassa. Hoofdstuk 1 geeft een korte historische achtergrond over onderzoek naar lichaamssamenstelling en skeletspiermassa, veelvoorkomende meetmethodes worden beschreven, en de huidige literatuur over de relatie tussen lage skeletspiermassa en nadelige behandeluitkomsten wordt uiteengezet. Tot slot worden de rationale en de doelen van dit proefschrift beschreven.

In Deel II van dit proefschrift wordt onderzoek beschreven naar de meetmethode voor skeletspiermassa bij HHPCC patiënten. In Hoofdstuk 2 wordt een validatiestudie beschreven van een eerder gepubliceerde meetmethode voor lage skeletspiermassa bij HHPCC patiënten. De methode van Swartz et al, waarbij skeletspiermassa wordt gemeten op het niveau van cervicale wervel C3, wordt gecorreleerd met een meting van skeletspiermassa op het niveau van lumbale wervel L3, wat in de algemene oncologische literatuur de meest beschreven methode is. De resultaten van deze studie laten zien dat er een goede correlatie is tussen skeletspieroppervlakte op niveau C3 en niveau L3 $(r=0.75$, $\mathrm{p}<0.01$ ). Wanneer de multivariabele predictie formule van Swartz et al wordt gebruikt om skeletspieroppervlakte op niveau van $L 3$ te voorspellen op basis van skeletspieroppervlakte op niveau van $\mathrm{C} 3$, is de correlatie tussen voorspelde skeletspieroppervlakte op niveau L3 en gemeten skeletspieroppervlakte op niveau L3 $0.82(p<0.01)$. De voorspelde en gemeten skeletspieroppervlakte op het niveau van $L 3$ werden genormaliseerd voor de lengte van de patiënt, om de lumbale skeletspiermassa index te bepalen (lumbale $\mathrm{SMI}$, in $\mathrm{cm}^{2} / \mathrm{m}^{2}$ ). Wanneer er gebruikt gemaakt werd van een door Wendrich et al gepubliceerde afkapwaarde voor lage skeletspiermassa van $<43.2 \mathrm{~cm}^{2} / \mathrm{m}^{2}$, was er een redelijke overeenkomst 
in de identificatie van patiënten met lage skeletspiermassa tussen de voorspelde lumbale SMI en gemeten lumbale SMI (Cohen's $\mathrm{k}=0.57$ ). De sensitiviteit van de voorspelde lumbale SMI om patiënten met een lage skeletspiermassa te identificeren bleef ondanks dit wel hoog (84.4\%). Deze studie laat zien dat een meting van skeletspiermassa op het niveau van C3 om patiënten met een lage skeletspiermassa te identificeren gebruikt kan worden als een goed alternatief voor een meting van skeletspiermassa op het niveau van L3. Hoofdstuk 3 behandelt een de interobservervariabiliteit van een meting van skeletspiermassa op het niveau van C3 op hoofd-hals CT beeldvorming. Voor dit onderzoek werden er CT scans van 54 HHPCC patiënten gebruikt om skeletspieroppervlakte op het niveau van $\mathrm{C} 3$ in te tekenen door 6 onafhankelijke observers, waarvan 3 artsonderzoekers, 1 hoofd-halschirurg, 1 hoofd-halsradiotherapeut en 1 hoofdhalsradioloog. De resultaten van dit onderzoek laten zien dat er een uitstekende overeenkomst is tussen de selectie van de juiste wervel $(\kappa 0.96)$ en redelijke overeenkomst bij de selectie van de specifieke CT slice (к 0.62). De correlatie tussen skeletspieroppervlakte bij alle observers was uitstekend (intraclass correlation coefficient $r=0.97, p<0.01$ ). Dit onderzoek laat zien dat de interobserver variabiliteit tussen een meting van skeletspiermassa tussen verschillende observers klein en klinisch verwaarloosbaar is. Hoofdstuk 4 presenteert een onderzoek naar de relatie tussen skeletspieroppervlakte gemeten op een enkele coupe ter hoogte van C3 en het totale lichaam skeletspiervolume zoals gemeten met whole body MRI. Dit onderzoek is een samenwerking met de Universiteit van Kiel in Duitsland, waar eerder onderzoek is verricht naar lichaamssamenstelling zoals gemeten met whole body MRI. ${ }^{9}$ Bij dit onderzoek werd gebruik gemaakt van whole body MRI's van gezonde vrijwilligers. De resultaten van dit onderzoek laten zien dat er een uitstekende correlatie is tussen skeletspieroppervlakte op niveau C3 en totale lichaam skeletspiervolume $(r=0.89, p<0.01)$. Een multivariabele predictie formule met daarin spieroppervlakte ter hoogte van C3, lengte, gewicht en geslacht, kon het totale lichaam skeletspiervolume zeer betrouwbaar worden geschat $(r=0.94, R 2=0.88 ; p<0.01)$. Dit onderzoek laat zien dat er een uitstekende correlatie is tussen skeletspieroppervlakte op niveau C3 en totale lichaam skeletspiervolume. Skeletspieroppervlakte op niveau C3 kan gebruikt worden om het totale lichaam skeletspiervolume te schatten.

Deel III van dit proefschrift beschrijft de predictieve en prognostische waarde van lage skeletspiermassa, gemeten op CT beeldvorming van het hoofdhals gebied op niveau C3, bij patiënten met HHPCC. In Hoofdstuk 5 wordt een onderzoek beschreven naar de predictieve en prognostische waarde van lage skeletspiermassa bij patiënten $(n=153)$ met lokaal gevorderd HHPCC die worden behandeld met primaire chemoradiatie met hoge dosis cisplatin, te weten $100 \mathrm{mg} / \mathrm{m}^{2}$ in 3 driewekelijkse kuren gecombineerd met 70 Gy radiotherapie in 35 fracties. In dit onderzoek hadden $54.9 \%$ van de patiënten een lage skeletspiermassa. Patiënten met een lage skeletspiermassa hadden significant vaker dosis-limiterende toxiciteit dan patiënten met een normale skeletspiermassa $(35.7 \%$ versus $10.1 \%, p<0.01)$. Lage skeletspiermassa en een mild verlaagde nierfunctie met een gemeten eGFR tussen de 60 en 70 waren beide onafhankelijke voorspellers voor het optreden van dosis-limiterende toxiciteit. Lage skeletspiermassa was geen significante voorspeller van een slechtere overleving ( $\mathrm{HR}$ 1.23, $\mathrm{p}=0.46)$. Het optreden van dosis-limiterende toxiciteit was wel voorspellend voor een slechtere overleving ( $H R$ 2.11, $p=0.02$ ). Hoofdstuk 6 presenteert een onderzoek bij 235 patiënten die een totale laryngectomie hebben ondergaan als behandeling van een larynx- of hypofarynxcarcinoom, of vanwege een afunctionele larynx na eerdere behandeling van hoofd-halskanker. In dit onderzoek werd gekeken naar de associatie tussen lage skeletspiermassa en het optreden van een faryngocutaan fistel, als ook lengte van ziekenhuisopname en overleving na totale laryngectomie. Er was bij $46.4 \%$ van de patiënten sprake van lage skeletspiermassa. De resultaten van dit onderzoek laten zien dat bij patiënten met preoperatieve lage skeletspiermassa vaker een faryngocutaan fistel optreedt dan patiënten met normale skeletspiermassa (34.9\% versus $20.6 \%)$. In multivariaat analyse was een lage skeletspiermassa een significante voorspeller voor het optreden van een faryngocutaan fistel (HR 1.95, $p=0.04$ ). Patiënten met lage skeletspiermassa verbleven significant langer in het ziekenhuis dan patiënten met normale skeletspiermassa (mediaan 17 versus 14 dagen, $p<0.01$ ). De totale overleving na totale laryngectomie was significant slechter bij patiënten met een lage skeletspiermassa dan bij patiënten met een normale skeletspiermassa (5-jaarsoverleving $32.1 \%$ versus $61.1 \%, \mathrm{p}<0.01)$. In Hoofdstuk 7 wordt een onderzoek gepresenteerd naar de overleving van oudere HHPCC patiënten met en zonder sarcopenie. Sarcopenie is een geriatrisch syndroom waarbij er sprake is van een lage skeletspiermassa én lage spierfunctie of spierkracht. Eerder onderzoek bij oudere patiënten laat zien dat het verlies van skeletspiermassa en spierfunctie en spierkracht niet lineair is, maar dat de combinatie hiervan voorspellend is voor slechter functioneren en sneller overlijden in de algehele geriatrische populatie. In dit onderzoek wordt sarcopenie gedefinieerd als een lage skeletspiermassa zoals gemeten op $\mathrm{CT}$ beeldvorming ter hoogte van $\mathrm{C} 3$, en de aanwezigheid van lage spierkrach gemeten met handknijpkracht of lage spierfunctie gemeten met een 4 meter looptest, zoals ook eerder gedefinieerd door de European working group on sarcopenia in older people (EWGSOP). ${ }^{10}$ In dit onderzoek is een heterogene groep 
van oudere (leeftijd $>70$ jaar) HHPCC patiënten geïncludeerd $(n=85)$, waarvan 71 patiënten een behandeling ondergingen met curatieve intentie. Van de 85 patiënten had $81.2 \%$ lage skeletspiermassa, 58.8\% lage spierkracht en $68.2 \%$ lage spierfunctie. In totaal hadden $48.2 \%$ patiënten sarcopenie. Lage skeletspiermassa, spierkracht en spierfunctie waren afzonderlijk niet voorspellend voor een kortere overleving. Sarcopenie was wel voorspellend voor een kortere overleving in patiënten met een curatief behandeltraject (HR 2.80, $p=0.03$ ). Hoofdstuk 8 beschrijft een onderzoek naar de prognostische waarde van lage skeletspiermassa en $\bigotimes$ sarcopene obesitas $\bigotimes$ bij patiënten met een orofarynxcarcinoom. Sarcopene obesitas is de combinatie van een lage skeletspiermassa en overgewicht. Het orofarynxcarcinoom is een van de meest voorkomende vormen van het HHPCC, waarbij met name de hoog-risico HPV-geassocieerde orofarynxcarcinomen steeds vaker voorkomen. De HPV-geassocieerde orofarynxncarcinomen hebben een veel betere prognose van de niet HPV-geassocieerde orofarynxcarcinomen. In dit onderzoek zijn 216 patiënten geïncludeerd, waarvan 92 met hoog-risico HPV. In $64.8 \%$ van de patiënten was er sprake van lage skeletspiermassa; maar $6 \%$ van de patiënten had sarcopene obesitas. Lage skeletspiermassa en sarcopene obesitas waren prognostisch voor een kortere algehele overleving (HR 1.76, $p=0.04$ en HR 2.44, $p=0.04$ respectievelijk). Sarcopene obesitas was onafhankelijk van HPVstatus voorspellend voor een kortere algehele overleving en ziektevrije overleving (HR 3.16, $p=0.01$ en HR 3.49, $p=0.04$ )

In de voorgaande hoofdstukken is gefocust op de relatie tussen lage skeletspiermassa zoals gemeten op routine vervaardigde CT beeldvorming en negatieve behandeluitkomsten. Er zijn echter nog meer applicaties te bedenken waar routine vervaardigde CT beeldvorming gebruikt kan worden om aanvullende informatie te verkrijgen over een patiënt. Een van deze applicaties is het in kaart brengen van de arteriële calcificaties die zichtbaar zijn op CT, als indirecte maat voor algeheel cardiovasculair lijden. In onderzoeken bij patiënten met slokdarmkanker en colorectaal kanker waren arteriële calcificaties op CT beeldvorming voorspellend voor het optreden van postoperatieve complicaties."

Hoofdstuk 9 beschrijft een onderzoek naar de relatie tussen locoregionale arteriële calcificaties op CT beeldvorming en faryngocutaan fistelvorming bij 224 patiënten die een totale laryngectomie hebben ondergaan. Arteriële calcificaties werden in kaart gebracht op 10 anatomische locaties in het hoofd-halsgebied en de bovenste thoracale regio. Dit onderzoek laat zien dat arteriële calcificaties zeer frequent voorkomen bij patiënten die een totale laryngectomie ondergaan; bij maar 1.3\% van de patiënten werden geen arteriële calcificaties waargenomen en bij $7.3 \%$ werden hoogstens milde calcificaties gezien. Een hogere cumulatieve calcificatie score op een schaal van 0 tot 30 was significant geassocieerd met het optreden van een faryngocutaan fistel (OR 1.06, $p=0.03$ )

In Deel IV van dit proefschrift wordt gekeken naar mogelijke toepassingen van lage skeletspiermassa in de klinische praktijk om de behandeling van HHPCC patiënten te verbeteren. In Hoofdstuk 10 wordt een studie protocol beschreven voor een prospectieve studie om het verband tussen lage skeletspiermassa en het optreden van cisplatin dosis-limiterende toxiciteit bij HHPCC patiënten te verklaren. Cisplatin is een chemotherapeuticum wat zich verdeelt in de waterhoudende weefsels in het lichaam en wordt gedoseerd op basis van het lichaamsoppervlakte (body surface area, BSA) van een patiënt. Patiënten met een lage skeletspiermassa en normale of hoge vetmassa hebben hierbij mogelijk een kleiner verdelingsvolume voor de cisplatin dan op basis van BSA wordt berekend. Reeds bekend is dat er een matige correlatie is tussen BSA en cisplatin farmacokinetiek. ${ }^{12}$ De hypothese voor het vaker voorkomen van cisplatin dosislimiterende toxiciteit bij patiënten met lage skeletspiermassa, is dat patiënten met een lage skeletspiermassa een relatieve overdosering cisplatin krijgen. Deze hypothese is nog niet getoetst. Als de hypothese klopt, kunnen de resultaten van dit onderzoek gebruikt worden om een alternatief doseringsregime voor cisplatin te formuleren, met als doel minder dosis-limiterende toxiciteit, waardoor meer patiënten de volledige behandeling met cisplatin kunnen voltooien. Tot slot wordt er in Hoofdstuk 11 een samenvatting gegeven van de bovengenoemde onderzoeken in dit proefschrift, en wordt er een kader geschetst voor huidige en toekomstige onderzoeken en toepassingen van lage skeletspiermassa bij de behandeling van patiënten met HHPCC. 


\section{Referenties}

1. Sung H, Ferlay J, Siegel RL, et al. Global Cancer Statistics 2020: GLOBOCAN Estimates of Incidence and Mortality Worldwide for 36 Cancers in 185 Countries. CA Cancer J Clin. 2021;71 (3):209-249. doi:10.3322/caac.21660

2. Paydarfar JA, Birkmeyer NJ. Complications in Head and Neck Surgery. Arch Otolaryngol Neck Surg. 2006;132(1):67. doi:10.1001/archotol.132.1.67

3. Szturz P, Wouters K, Kiyota N, et al. Low-Dose vs. High-Dose Cisplatin: Lessons Learned From 59 Chemoradiotherapy Trials in Head and Neck Cancer. Front Oncol. 2019;9. doi:10.3389/ fonc.2019.00086

4. Rier HN, Jager A, Sleijfer S, Maier AB, Levin M. The Prevalence and Prognostic Value of Low Muscle Mass in Cancer Patients: A Review of the Literature. Oncologist. 2016;21 (11):1396-1409. doi:10.1634/theoncologist.2016-0066

5. Prado $\mathrm{CM}$, Lieffers JR, McCargar LJ, et al. Prevalence and clinical implications of sarcopenic obesity in patients with solid tumours of the respiratory and gastrointestinal tracts: a population-based study. Lancet Oncol. 2008;9(7):629-635. doi:10.1016/S1470-2045(08)701530

6. Shen W, Punyanitya M, Wang Z, et al. Total body skeletal muscle and adipose tissue volumes: estimation from a single abdominal cross-sectional image. J Appl Physiol. 2004;97(6):23332338. doi:10.1152/japplphysiol.00744.2004

7. Swartz JE, Pothen AJ, Wegner I, et al. Feasibility of using head and neck CT imaging to assess skeletal muscle mass in head and neck cancer patients. Oral Oncol. 2016;62:28-33. doi:10.1016/j. oraloncology.2016.09.006

8. Wendrich AW, Swartz JE, Bril Sl, et al. Low skeletal muscle mass is a predictive factor for chemotherapy dose-limiting toxicity in patients with locally advanced head and neck cancer. Oral Oncol. 2017;71:26-33. doi:10.1016/j.oraloncology.2017.05.012

9. Schweitzer L, Geisler C, Pourhassan M, et al. What is the best reference site for a single MRI slice to assess whole-body skeletal muscle and adipose tissue volumes in healthy adults?1. Am J Clin Nutr. 2015;102(1):58-65. doi:10.3945/ajcn.115.111203

10. Cruz-Jentoft AJ, Bahat G, Bauer J, et al. Sarcopenia: revised European consensus on definition and diagnosis. Age Ageing. 2019:48(1):16-31. doi:10.1093/ageing/afy169

11. Borggreve AS, Goense L, van Rossum PSN, van Hillegersberg R, de Jong PA, Ruurda JP. Generalized cardiovascular disease on a preoperative CT scan is predictive for anastomotic leakage after esophagectomy. Eur J Surg Oncol. 2018;44(5):587-593. doi:10.1016/j.ejso.2018.01.225

12. De Jongh FE, Verweij J, Loos WJ, et al. Body-surface area-based dosing does not increase accuracy of predicting cisplatin exposure. J Clin Oncol. Published online 2001. doi:10.1200/ JCO.2001.19.17.3733 
Chapter 13

Curriculum Vitae about the author 


\section{Curriculum Vitae - about the author}

Sandra Isabella Bril was born the $19^{\text {th }}$ of October 1989 in Vries, a small village in northern Drenthe, The Netherlands. After graduating from secondary school in 2009, she started medical school at the Erasmus University in Rotterdam. Throughout her study, she worked as a medical assistant at the department of haemato-oncology at the Erasmus MC Cancer Center. After receiving her Bachelor's degree, she took part in a research project at the department of medical oncology. This sparked a research and clinical interest in oncology.

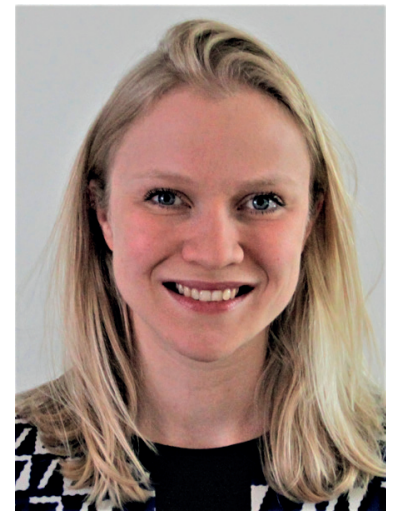

After graduating medical school in August 2016, she started as a PhD-student at the department of Head and Neck Surgical Oncology under the supervision of prof. dr. R. de Bree and dr. L.A. Devriese. She focused her research on the measurement method for skeletal muscle mass in head and neck cancer patients, using routinely performed imaging to extract additional data. She also focused on the relationship between radiologically assessed skeletal muscle mass and clinical outcomes in head and neck cancer patients. She presented her research at several national and international congresses, and she spent several weeks at the University of Kiel, Germany for a collaborative research project supervised by dr. C. Geisler. A highlight of her PhD career was setting up a prospective study to investigate the relationship between low skeletal muscle mass and chemotherapy dose limiting toxicity in head and neck cancer patients. From February 2018 onwards, she combined her PhD research with clinical work as a resident not in training at the department of Medical Oncology at the University Medical Center Utrecht.

In February 2019, she started as a resident not in training at the department of internal medicine at the Elisabeth-Tweesteden Ziekenhuis in Tilburg. In January 2021, she started her residency training in internal medicine at the ElisabethTweesteden Ziekenhuis under the supervision of dr. M.E.E. van Kasteren (ElisabethTweesteden Ziekenhuis) and dr. A.M. van Zandbergen (Erasmus Medical Center). 


\section{Chapter 14}

List of publications 


\section{List of publications}

SI Bril, AJ Pothen, R de Bree. "Sarcopenie bij patiënten met hoofd-halskanker." Nederlands Tijdschrift voor Oncologie. 2017; 14(2).

AW Wendrich, JE Swartz, SI Bril, I Wegner, A de Graeff, EJ Smid, R de Bree, AJ Pothen. "Low skeletal muscle mass is a predictive factor for chemotherapy doselimiting toxicity in patients with locally advanced head and neck cancer." Oral Oncology 2017 Aug;71:26-33.

SI Bril, AW Wendrich, JE Swartz, I Wegner, F Pameijer, EJ Smid, GH Bol, AJ Pothen, R de Bree. Interobserver agreement of skeletal muscle mass measurement on head and neck CT imaging at the level of the third cervical vertebra. European Archives of Oto-Rhino-Laryngology 2019 Apr;276(4):1175-1182.

JF Petersen, TF Pezier, JM van Dieren, V van der Noort, T van Putten, SI Bril, LM Janssen, R Dirven, MWM van den Brekel, R de Bree. Dilation after laryngectomy: Incidence, risk factors and complications. Oral Oncology 2019 Apr;91:107-112.

N Chargi, SI Bril, MH Emmelot-Vonk, R de Bree. Sarcopenia is a prognostic factor for overall survival in elderly patients with head-and-neck cancer. European Archives of Oto-Rhino Laryngology 2019 May;276(5):1475-1486.

SI Bril, TF Pezier, BM Tijink, LM Janssen, WW Braunius, R de Bree. Preoperative low skeletal muscle mass as a risk factor for pharyngocutaneous fistula and decreased overall survival in patients undergoing total laryngectomy. Head Neck 2019 Jun;41(6):1745-1755.

N Chargi, SI Bril, PA de Jong, MH Emmelot-Vonk, R de Bree. Reply to comment on "Sarcopenia is a prognostic factor for overall survival in elderly patients with head and neck Cancer". European Archives of Oto-Rhino-Laryngology 2019 Sep;276(9):2631-2633. Letter to the editor

RT Karsten, A Al-Mamgani, SI Bril, S Tjon-A-Joe, L van der Molen, JP de Boer, FJM Hilgers, LE Smeele, MWM van den Brekel, MM Stuiver. Sarcopenia, a strong determinant for prolonged feeding tube dependency after chemoradiotherapy for head and neck cancer. Head Neck 2019 Nov;41(11):4000-4008.
N Chargi, SI Bril, JE Swartz, I Wegner, SM Willems, R de Bree. Skeletal muscle mass is an imaging biomarker for decreased survival in patients with oropharyngeal squamous cell carcinoma. Oral Oncology 2020 Feb;101:104519.

R de Bree, JE Swartz, SI Bril, N Chargi, I Wegner, EJ Smid. Skeletal muscle mass measurements using head and neck CT imaging in head and neck cancer patients. Radiotherapy and Oncology. 2021 Aug;161:72-73. Letter to the editor

SI Bril, MA van Beers, N Chargi, N Carrillo, EJ Smid, JW Dankbaar, R de Bree Skeletal muscle mass at C3 is a strong predictor for skeletal muscle mass at L3 in sarcopenic and non-sarcopenic patients with head and neck cancer. Oral Oncology 2021 Oct 6;122:105558. Letter to the editor

SI Bril, A Al-Mamgani, N Chargi, P Remeijer, LA Devriese, JP de Boer, R de Bree. Pretreatment low skeletal muscle mass is associated with chemotherapy dose-limiting toxicity in head and neck cancer patients undergoing primary chemoradiotherapy with high dose cisplatin. Accepted for publication in Head Neck (October 2021)

SI Bril*, N Chargi*, AW Wendrich, I Wegner, GH Bol, EJ Smid, PA de Jong, LA Devriese, $\mathrm{R}$ de Bree. Validation of skeletal muscle mass assessment at the level of the third cervical vertebra in patients with head and neck cancer. Submitted

\section{* Contributed equally}

SI Bril, C Geisler, L Schweitzer, A Bosy Westphal, PA de Jong, R de Bree, MJ Müller. Total skeletal muscle volume estimation from a single magnetic resonance imaging slice of the head and neck area in healthy adults. Submitted

SI Bril, N Chargi, TF Pezier, BM Tijink, WW Braunius, EJ Smid, PA de Jong, R de Bree. Arterial calcification on preoperative CT imaging is a risk factor for pharyngocutaneous fistula formation after total laryngectomy. Accepted for publication in Head Neck, October 2021

\section{Not related to this thesis}

RRJ Coebergh van den Braak, AM Sieuwerts, R Kandimalla, ZS Lalmahomed, SI Bril A van Galen, M Smid, K Biermann, JH van Krieken, WP Kloosterman, JA Foekens, A Goel, JWM Martens, JNM IJzermans. "High mRNA expression of splice variant SYK short correlates with hepatic disease progression in chemonaive lymph node negative colon cancer patients." PLoS One. 2017 Sep 28;12(9):e0185607. 
RRJ Coebergh van den Braak, AM Sieuwerts, ZS Lalmahomed, M Smid, SM Wilting, SI Bril, S Xiang, M van der Vlugt-Daane, V de Weerd, A van Galen, K Biermann, JHJM van Krieken, WP Kloosterman, JA Foekens, JWM Martens, JNM IJzermans. Confirmation of a metastasis-specific microRNA signature in primary colon cancer. Sci Rep. 2018 Mar 27;8(1):5242.

M Smid, RRJ Coebergh van den Braak, HJG van de Werken, J van Riet, A van Galen, $\checkmark$ de Weerd, M van der Vlugt-Daane, SI Bril, ZM Lalmahomed, WP Kloosterman, SM Wilting, JA Foekens, JNM IJzermans, JWM Martens, AM Sieuwerts. Gene length corrected trimmed mean of M-values (GeTMM) processing of RNA-seq data performs similarly in intersample analyses while improving intrasample comparisons. BMC Bioinformatics. 2018 Jun 22;19(1):236. 
Chapter 15

Dankwoord 


\section{Dankwoord}

Na ruim 5 jaar is mijn proefschrift eindelijk daar. Dit proefschrift zou niet geschreven zijn zonder de hulp en ondersteuning van een hoop mensen, die ik daar zeer dankbaar voor ben.

Beste Prof. dr. de Bree, "hoi Remco", allereerst dank voor de kans om na mijn oudste coschap in het UMC Utrecht direct door te stromen in dit promotie onderzoek. Het is fantastisch om bij en met jou onderzoek te doen, ik had me geen betere promotor kunnen wensen. Jouw motivatie en drive voor het verbeteren van de zorg voor hoofd-halskankerpatiënten is ongeëvenaard. Je bent altijd enthousiast en geïnteresseerd, en hebt me alle vrijheid gegeven tijdens het onderzoek om nieuwe projecten op te zetten en nieuwe ideeën uit te werken. Mijn onderzoek is zonder twijfel met ups en downs gegaan, maar dankzij jouw ondersteuning is dit proefschrift toch tot een goed einde gekomen.

Beste dr. Devriese, beste Lot, jouw oprechte interesse en opbouwende kritische insteek heeft de onderzoekslijn sarcopenie bij hoofd-halskankerpatiënten naar een hoger niveau getild. Het was ontzettend leuk en leerzaam om samen met jou een studie op te zetten, van alleen een idee tot aan een lopend onderzoek. Naast dat je co-promotor bent, heb ik ook onder jouw supervisie mijn eerste stappen gezet als arts-assistent bij de early clinical trial unit van de afdeling oncologie van het UMC Utrecht. Je hebt misschien eerder dan ikzelf doorgehad dat er meer een internist in mij schuilt dan een KNO-arts, waarvoor ik je nog altijd dankbaar ben.

Beste leden van de beoordelings- en promotiecommissie, geachte prof. dr. Witteveen, prof. dr. van den Brekel prof. dr. Hendrikse, prof. dr. Emmelot-Vonk, prof. dr. Vermorken, prof. dr. Zuur, prof. dr. Koopman en dr. Steenbakkers, allen veel dank dat $u$ de tijd heeft genomen voor de kritische beoordeling van dit proefschrift.

Beste hoofd-hals chirurgen en fellows hoofd-hals chirurgie van het UMC Utrecht, dank voor jullie bijdrages aan dit onderzoek en de goede feedback op meerdere manuscripten. Ook veel dank aan de hoofd-hals radiotherapeuten, radiologen, pathologen en onderzoekers die direct of indirect bij de verschillende studies betrokken zijn geweest.

Lieve Bea, dank voor de ondersteuning tijdens mijn promotietraject; dat is van onschatbare waarde geweest. Dank voor je organisatorisch vermogen om alles in goede banen te leiden, maar ook je menselijkheid en medeleven als het even niet mee zat. Zelfs nu bij de laatste loodjes ben je betrokken. Je bent voor alle promovendi van Remco een grote steun!

Beste collega onderzoekers van de hoofd-hals chirurgische oncologie, dank voor de samenwerking en de mooie tijd in het $\mathrm{Q}$ gebouw. Najiba, het is ongelooflijk hoe veel werk jij hebt verzet in zo'n korte tijd. Ik ken weinig mensen die zo gedreven zijn als jij. Je gaat een fantastische MKA-chirurg worden. Inne, Boris, Joost, Lilian, Emma en Jorine, dank voor de koffietjes en de pubquizzen in Utrecht met teams de Hoofdzaak en de Halszaak. De congressen in Barcelona, Rome en Buenos Aires met jullie waren hoogtepunten in mijn onderzoekstijd. Justin en Anne, jullie hebben de eerste puzzelstukjes van het onderzoek gelegd; dank dat ik op jullie werk mocht voortborduren. Maartje, ik weet zeker dat je de sarcopenie onderzoekslijn weer verder gaat uitdiepen en nieuwe inzichten gaat vinden.

Beste dr. Al-Mamgani, dank voor de mogelijkheid om samen te werken op het onderzoek naar cisplatinum dosis-limiterende toxiciteit bij chemoradiatie. Het heeft even geduurd, maar inmiddels is het resulterende artikel bijna gepubliceerd.

Dear dr. Geisler, dear Corinna, thank you very much for the opportunity to collaborate on our research project. Your database of whole-body MRI scans was invaluable for us and I am very grateful that you welcomed me. Also thank you for showing me around, sight-seeing advice and generally making me feel very welcome in Kiel.

Beste oncologen en fellows oncologie van het UMC Utrecht, dank dat ik als ANIOS binnen jullie vakgebied ervaring mocht opdoen, ik heb me zeer welkom gevoeld. Een speciale dank aan de stafleden van de early clinical trial unit. Lot, Els, Eelke en Filip, ik heb het ontzettend leuk gevonden om onder jullie supervisie te leren wat er allemaal komt kijken bij het uitvoeren van vroeg klinische studies. Ik vond het super interessant om een stukje te leren van wat er gaande is binnen de ontwikkeling van nieuwe geneesmiddelen en de optimalisatie van bestaande.

Beste prof. dr. Huitema en dr. Frederix, dank voor jullie hulp bij de subsidie aanvraag voor de Platisma studie. Zonder jullie kennis was het niet gelukt.

Beste medewerkers van het trial bureau medische oncologie, dank voor al jullie hulp en ondersteuning bij alle vroeg klinische studies, en ook niet in de laatste plaats bij de Platisma studie. Ellis, gelukkig kan jij wel een weg vinden 
in alle formulieren en documenten die komen kijken bij het opzetten van een studie, en was je altijd bereid om last-minute mij te ondersteunen. Beste tria verpleegkundigen en verpleegkundigen van de afdeling oncologie, dank voor a jullie hulp en flexibiliteit bij de uitvoering van de Platisma studie en samenwerking in de kliniek. Ik vond het prettig samenwerken met jullie.

Beste medewerkers van het UMC Utrecht Cancer Center trial bureau, en specifiek Antoinette, dank voor jullie hulp bij al mijn METC aanvragen en andere organisatorische dingen rondom mijn onderzoek. Jullie eeuwige geduld, behulpzaamheid en kennis van zaken zorgden ervoor dat ik me geen zorgen heb hoeven maken hierover.

Beste stafleden van de afdeling interne geneeskunde in het ElisabethTweesteden Ziekenhuis, dank voor het warme welkom in Tilburg en voor het fijne opleidingsklimaat in het ETZ. Na anderhalf jaar bikkelen is er hopelijk snel tijd voor wat meer ontspanning: ik kijk uit naar de langverwachte ski- en zeilweekenden! Beste dr. van Kasteren, beste Marjo, dank dat ik onder jouw supervisie aan mijn opleiding tot internist heb mogen starten.

Lieve arts-assistenten en oud-assistenten interne geneeskunde en beschouwende pool ETZ, ik vind dat we een fantastische groep hebben in Tilburg wat het werk extra leuk maakt. De laatste tijd was het meer work hard dan play hard, maar het afgelopen assistentenweekend heeft laten zien dat we de gezelligheid niet zijn verleerd. Een speciale dank aan de promotietraject-ervaringsdeskundigen, die me eraan bleven herinneren dat het uiteindelijk wel goed komt. Ook een bijzondere shout-out naar Fred's Fietsclub, het almaar groter wordende clubje fietsende internisten-en-aanverwanten. Wat begon als een avondje midden in coronatijd digitaal fietsen, heeft inmiddels al geresulteerd in meerdere mooie fietstochten door het Brabantse land. Het was fijn om in coronatijd elkaar te kunnen spreken buiten het ziekenhuis over een gedeelde hobby. lk hoop dat er nog veel mooie fietstochten gaan volgen.

Lieve Sam, dank dat je mijn paranimf wil zijn. Je hebt me al gauw op sleeptouw genomen nadat ik in Tilburg kwam werken, binnen en buiten het ziekenhuis; ik kan zeker zeggen dat ik me hierdoor meer thuis ben gaan voelen in Tilburg.

Lieve familie en vrienden, dank voor de vriendschappen, gezelligheid en betrokkenheid de afgelopen jaren. Dank voor alle gesprekken over het onderzoek doen en werken in het ziekenhuis, maar vooral ook dank voor alle tijden dat het daar niet over ging. Dank voor alle etentjes, borrelavonden en feestjes, en voor de uitjes en weekendjes weg, voor de spelletjes avonden, skivakanties of rondjes fietsen. Ik heb de afgelopen jaren niet altijd zoveel tijd of aandacht gehad voor jullie als ik had gewild. Ik hoop dat er nog veel mooie momenten samen zullen volgen.

Lieve Liesbeth, Daphne en Stijn, ik kan me geen fijnere schoonfamilie wensen dan jullie. De laatste jaren zijn zwaar geweest met de ziekte en het verlies van Ad. Ik hoop dat de komende jaren veel gezondheid en geluk gaan brengen.

Lieve Lianne en Nick, ik kan niet anders zeggen dan dat jullie het prima voor elkaar hebben daar in het hoge noorden. Ik ben trots op hoe jullie samen jullie leven georganiseerd hebben ondanks jullie beider drukke banen. Dank voor jullie betrokkenheid al deze jaren. Lianne, nog altijd mijn kleine zusje maar inmiddels ook al 28, ik vind het super stoer dat je scheikundig consultant bent. Ik kijk uit naar onze gezamenlijke etentjes in te dure restaurants, avonden stand up comedy, of gewoon biertjes drinken en slap ouwehoeren. Dankjewel dat je mijn paranimf wil zijn.

Lieve René, het is ontzettend verdrietig dat jij er zo plots niet meer was. Ik mis je, en ben dankbaar voor hoe goed jij al die jaren voor mam, Lianne en mij hebt gezorgd.

Lieve mam, dankjewel voor je onvoorwaardelijke steun. Je bent een voorbeeld in onafhankelijkheid en kansen pakken. Door jouw harde werk hebben Lianne en ik alle mogelijkheden gehad om zonder zorgen op te groeien en te studeren. Ik vind het knap hoe jij het leven weer kan oppakken en het plezier kan terugvinden na een tegenslag. Ik ben benieuwd naar al je toekomstplannen: een trekking in Tibet een nieuwe fietsvakantie, misschien wel leren motor rijden..? Ik hoop dat ik later net zo vitaal en ondernemend in het leven zal staan als jij.

Lieve Dennis, we leerden elkaar kennen tijdens het begin van onze onderzoekstijd. We hebben alle ups en downs van een promotietraject samen doorgemaakt, met als bizar hoogtepunt gelijktijdige, volledig ongerelateerde congressen in Buenos Aires waar we allebei naartoe mochten. Dank voor je hulp bij mijn inburgering in Tilburg, ik voel me inmiddels hier helemaal thuis (dè ge bedankt zèèt, dè witte) Dank voor je humor, je rust en je zorgzaamheid; gelukkig kan jij af en toe wel op de rem trappen wanneer ik dat vergeet. Ik kijk uit naar een zonnige toekomst samen in ons nieuwe huis met onze Frenkie. Ik houd van je. 